\title{
Atg21 restricts Atg8 lipidation to a novel vacuole-phagophore contact site
}

\author{
Dissertation
}

\author{
for the award of the degree \\ "Doctor rerum naturalium" \\ of the Georg-August-University Göttingen \\ within the doctoral program "Molecular Biology of Cells" \\ of the Georg-August-University School of Science (GAUSS)
}

submitted by

Lena Munzel

from Göttingen

Göttingen, 2018 


\section{Members of the Thesis Committee}

Prof. Dr. Michael Thumm

Department of Cellular Biochemistry

Center for Biochemistry and Molecular Cell Biology

University Medical Center Göttingen

Prof. Dr. Gerhard Braus

Department of Molecular Microbiology and Genetics

Institute of Microbiology and Genetics, Georg-August-University Göttingen Prof. Dr. Ralf Ficner

Department of Molecular Structural Biology

Institute of Microbiology and Genetics, Georg-August-University Göttingen

\section{Members of the Examination Board}

1st Referee and Supervisor: Prof. Dr. Michael Thumm

Department of Cellular Biochemistry

Center for Biochemistry and Molecular Cell Biology

University Medical Center Göttingen

2nd Referee: Prof. Dr. Gerhard Braus

Department of Molecular Microbiology and Genetics

Institute of Microbiology and Genetics, Georg-August-University Göttingen

\section{Further members of the Examination Board}

Prof. Dr. Ralf Ficner

Department of Molecular Structural Biology

Institute of Microbiology and Genetics, Georg-August-University Göttingen

Prof. Dr. Heike Krebber

Department of Molecular Genetics

Institute of Microbiology and Genetics, Georg-August-University Göttingen

Prof. Dr. Stefan Jakobs

Research Group Mitochondrial Structure and Dynamics

Department of NanoBiophotonics

Max Planck Institute for Biophysical Chemistry, Göttingen

Dr. Hans Dieter Schmitt

Department of Neurobiology

Max Planck Institute for Biophysical Chemistry, Göttingen

Date of oral examination: 09.01.2019 
Affidavit:

I hereby declare that the thesis entitled "Atg21 restricts Atg8 lipidation to a novel vacuole-phagophore contact site" has been written independently and with no other sources and aids than quoted.

Lena Munzel

Göttingen, November 2018 



\section{Content}

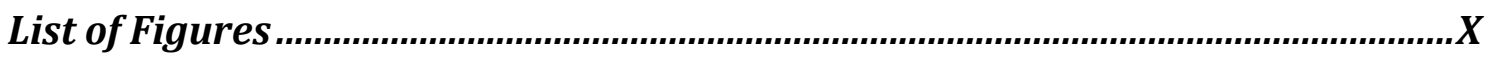

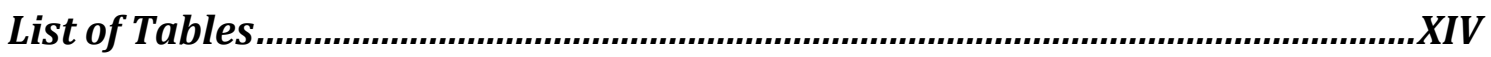

Abbreviation

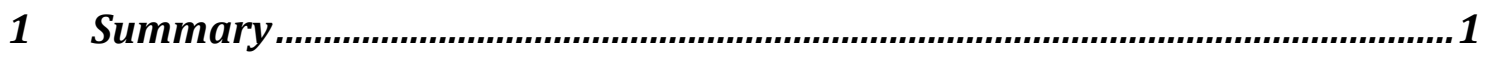

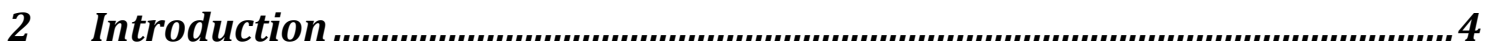

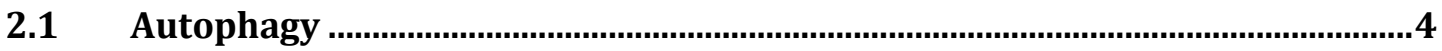

2.1.1 The yeast S. cerevisiae as a model organism to study autophagy …………......................... 5

2.1.2 Macroautophagy ...................................................................................................................

2.1.3 The Cytosol-to-vacuole targeting pathway as a model of selective autophagy ................ 8

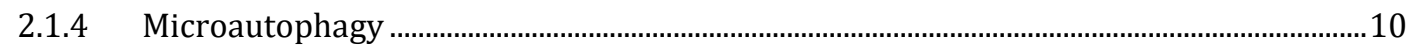

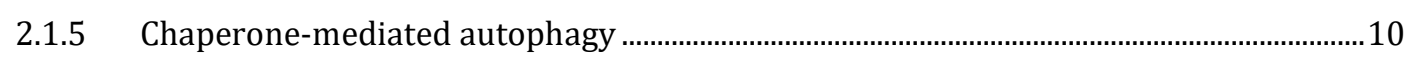

2.2 Induction of autophagy and the autophagic core machinery ….....................11

2.2.1 The Atg1 kinase complex and autophagy induction ………………………………………....11

2.2.2 Atg9-containing vesicles .................................................................................................14

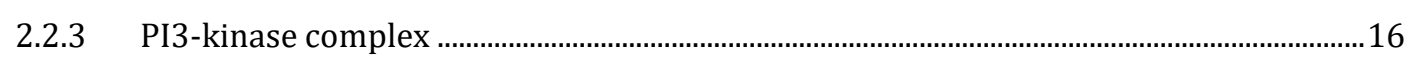

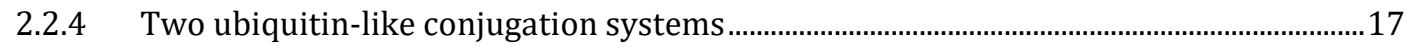

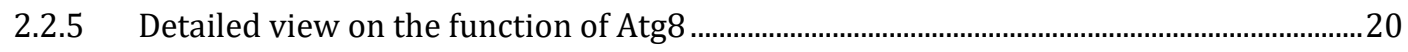

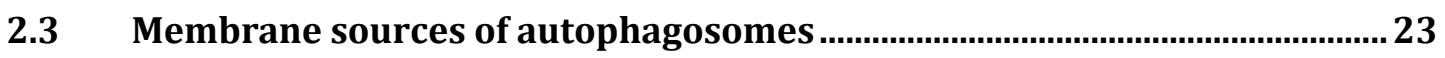

2.4 Autophagosome maturation and fusion with the vacuole.............................. 26

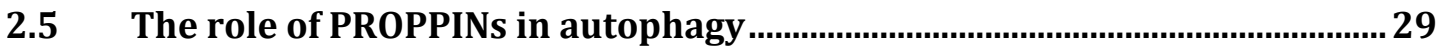

2.5.1 Structure and membrane binding of PROPPINs ....................................................................30

2.5.2 The functions of yeast PROPPINs ..........................................................................................

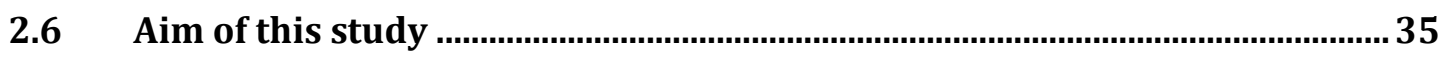

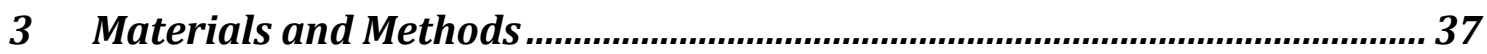

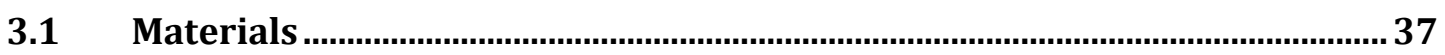

3.1.1 Yeast and bacteria strains.................................................................................................

3.1.1.1 Saccharomyces cerevisiae strains ..................................................................................3

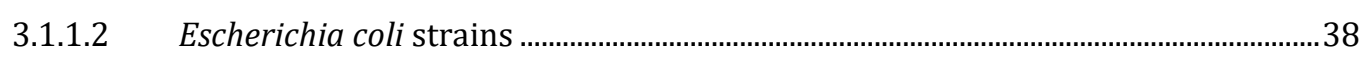

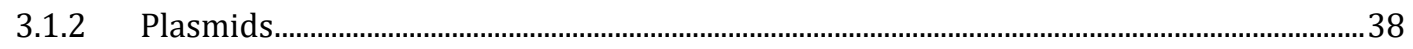

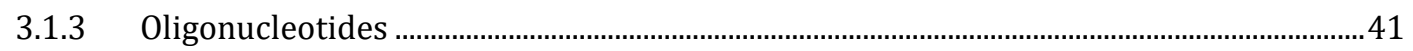




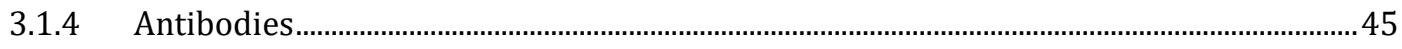

3.1.5 Commercially available kits and master mixes...............................................................45

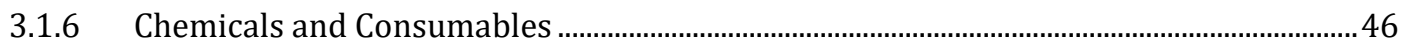

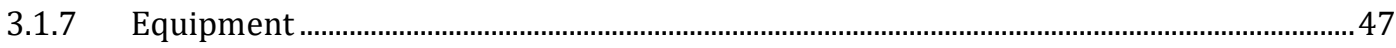

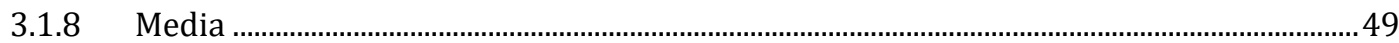

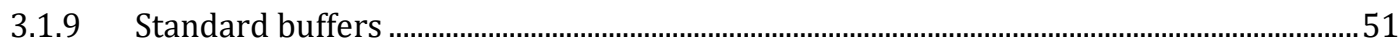

Cultivation conditions ............................................................................. 52

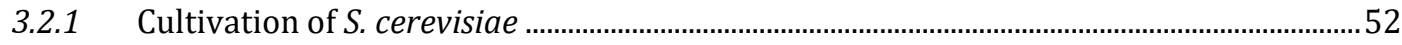

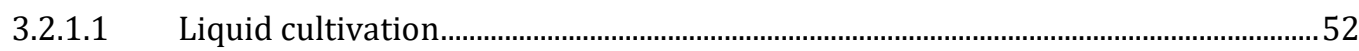

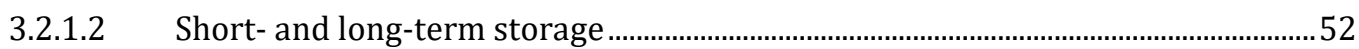

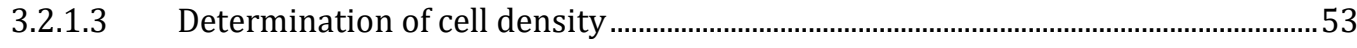

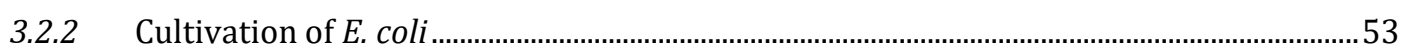

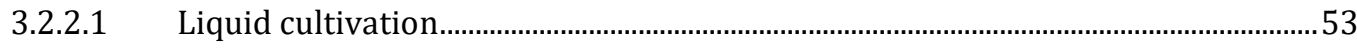

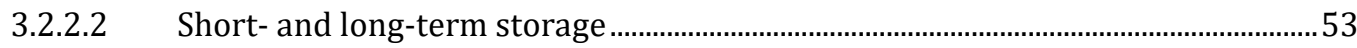

3.3 Molecular biological methods .....................................................................53

3.3.1 Isolation of chromosomal DNA from Yeast......................................................................... 53

3.3.2 Plasmid Isolation from E. coli............................................................................................... 54

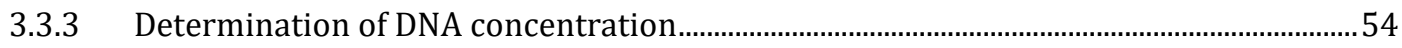

3.3.4 DNA agarose gel electrophoresis ..........................................................................................5 54

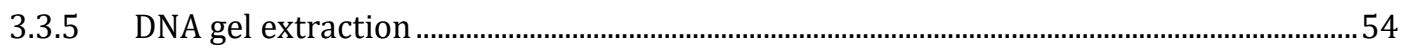

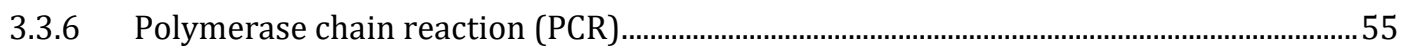

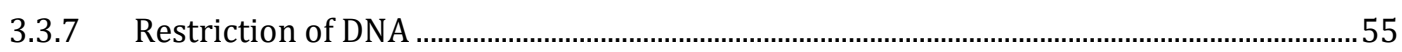

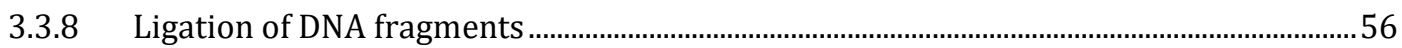

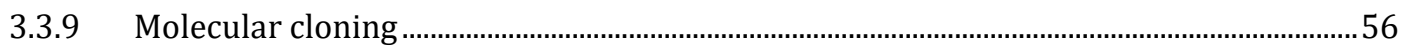

3.3.10 Plasmid construction ................................................................................................. 57

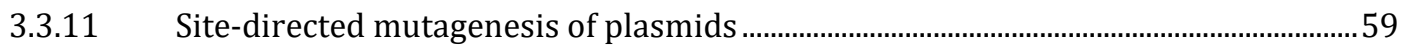

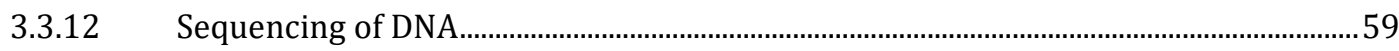

3.3.13 Preparation of chemically competent E. coli ...................................................................59

3.3.14 Transformation of plasmid DNA in chemically competent $E$. coli ..............................59

3.3.15 High efficiency transformation of DNA in yeast ..........................................................60

3.3.16 Transformation of plasmid DNA in yeast ("Quick and Dirty") ....................................60

3.3.17 Gene deletion and chromosomal tagging using homologous recombination ...........61

3.3.18 Isolation of plasmid DNA from yeast cells (plasmid rescue) ......................................61

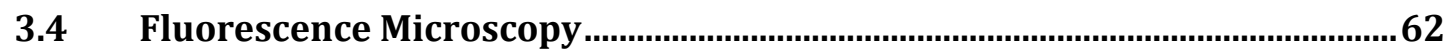

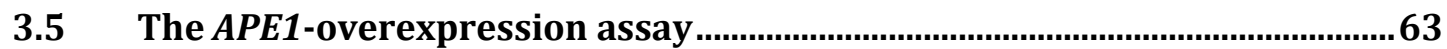

Fluorescence cross-correlation spectroscopy (FCCS) ................................63

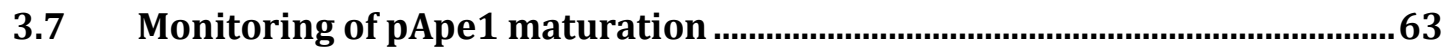




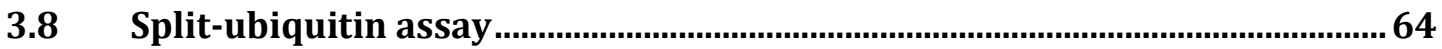

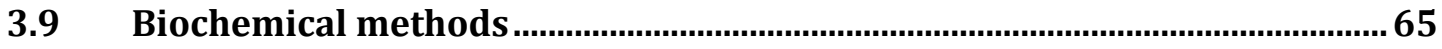

3.9.1 SDS-Polyacrylamide Gel Electrophoresis (SDS-PAGE) …………………………………....65

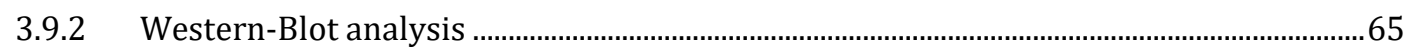

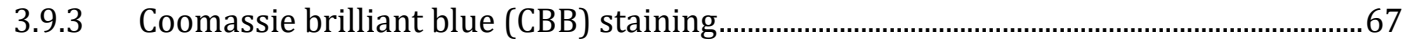

3.9.4 Alkaline lysis of yeast cells ..................................................................................................67

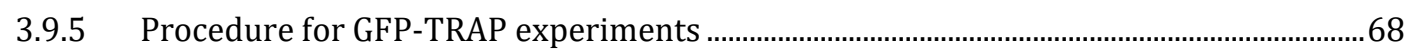

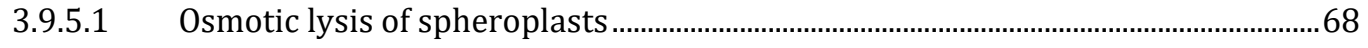

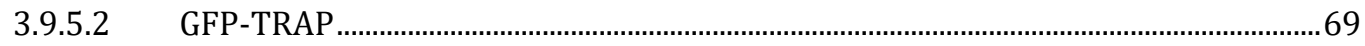

3.9.6 Proximity-dependent biotin identification (BioID) assay ..................................................69

3.9.6.1 Preparation of yeast cultures without SILAC ……………………………………….....70

3.9.6.2 Isolation of biotinylated proteins using Strep-Tactin ${ }^{\circledR}$ columns ...............................70

3.9.6.3 Preparation of yeast cultures for quantitative MS analysis ....................................

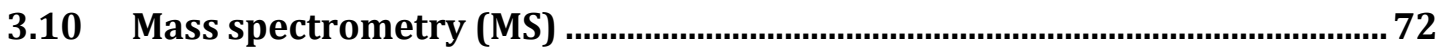

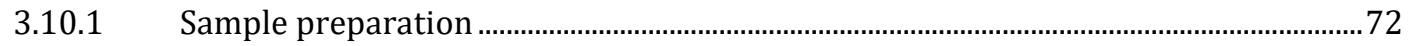

3.10.2 Liquid chromatography-mass spectrometry (LC-MS) analysis ....................................73

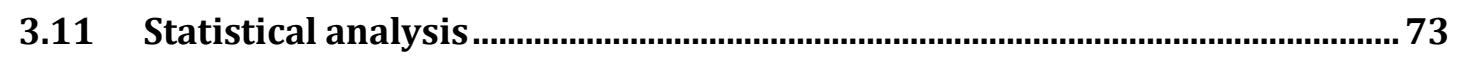

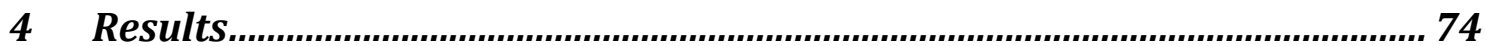

4.1 Detailed analysis of the interaction of Atg21 and Atg16 ................................74

4.1.1 Fine mapping of the interacting domains of Atg21 and Atg16 ........................................74

4.1.1.1 Identification of amino acids that potentially contribute to the interaction of

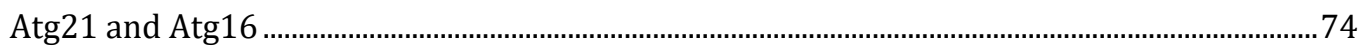

4.1.1.2 R151 of Atg21 directly interacts with D101 of Atg16 ...............................................79

4.1.1.3 Contribution of other amino acids to the interaction of Atg21 and Atg16 ..........86

4.1.2 Dynamics of the formation of the Atg21-Atg16 complex ..................................................90

4.1.2.1 Evaluating the interaction of Atg16 and Atg21 ${ }^{\text {FTTG }}$ using the GFP-TRAP

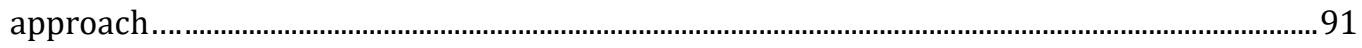

4.1.2.2 Analyzing the interaction of Atg16 and Atg21 FTTG by fluorescence crosscorrelation spectroscopy (FCCS).

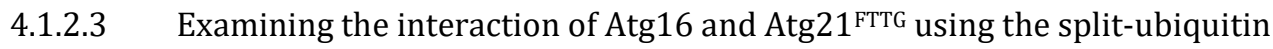
assay

4.1.2.4 Inspecting the interaction of Atg16 and Atg21 $1^{\text {FTTG }}$ with the help of the proximity-dependent biotin identification (BioID) assay

\subsection{Detailed analysis of the localization of autophagy related proteins at the} growing phagophore to investigate their role in autophagosome formation ......101

4.2.1 Establishing and optimizing the APE1-overexpression assay 102 
4.2.2 Localization of Atg21 at the growing phagophore under different growth conditions. 105

4.2.3 Localization of the Atg8 lipidation complex component Atg3 at the growing phagophore.

4.2.4 The distribution of the PI3-kinase complex, PI3P and the PROPPIN Atg18 at the growing phagophore in comparison to Atg21.

4.2.5 The contact site of the phagophore and the vacuolar membrane and the role of Vac8 in phagophore formation 114

4.2.5.1 Vac8 is enriched at the contact site between vacuole and phagophore...... 114

4.2.5.2 Vac8 participates in the formation of the phagophore 118

4.2.5.3 Upon deletion of VAC8 the contact between vacuolar membrane and phagophore is affected

4.2.5.4 Vac8 and Atg21 co-precipitate in the same complex. 124

4.2.6 Formation of a specialized vacuolar membrane domain at the contact site between vacuole and phagophore...

4.2.6.1 Vph1 is excluded from the contact site between vacuole and phagophore ...... 126

4.2.6.2 Further characterization of the identified vacuolar membrane domain............ 130

4.2.6.3 Atg21 is part of the vacuole-phagophore contact site 133

4.2.6.4 The ER is in close proximity to the edges of the growing phagophore but not to the vacuole-phagophore contact site. 134

4.3 Identification of unknown interaction partners of Atg21........................ 136

4.3.1 Establishing and optimizing the BioID assay ................................................................. 136

4.3.1.1 Construction of the BirA* fusion proteins ….................................................................... 138

4.3.1.2 BirA* fusion proteins complement the atg21 pApe1 maturation phenotype 139 4.3.1.3 Adapting the workflow of the BioID assay for the identification of Atg21 interacting proteins 140

4.3.2 Quantitative Analysis of the biotinylated proteins using SILAC 143

5

Discussion

\subsection{Structural and dynamical organization of the Atg8 lipidation complex.. 150}

5.1.1 Molecular details of the interacting domains of Atg16 and Atg21 and model of the overall structure of the Atg8 lipidation complex

5.1.2 Models of the membrane association of the Atg21-Atg16 complex .............................. 155

5.1.3 Dynamics of the Atg21-Atg16 complex formation... 159

\subsection{Determination of the precise localization of proteins at the growing} phagophore

5.2.1 Advantages and disadvantages of the APE1-overexpression assay and comparable approaches 
5.2.2 Under starvation conditions Atg21 restricts Atg8 lipidation to the contact site of vacuole and phagophore.

5.2.3 Atg21 shows an aberrant localization in stationary cells.................................................. 170

5.2.4 Identification of a novel vacuole-phagophore contact site................................................173

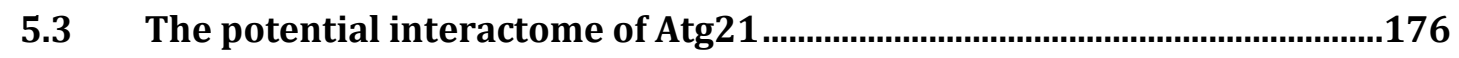

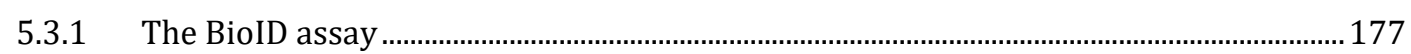

5.3.2 A potential role of Atg21 in the fusion of autophagosomes with the vacuole..............179

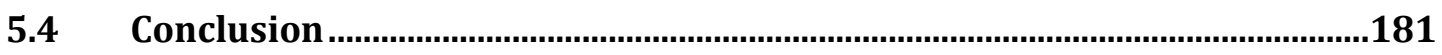

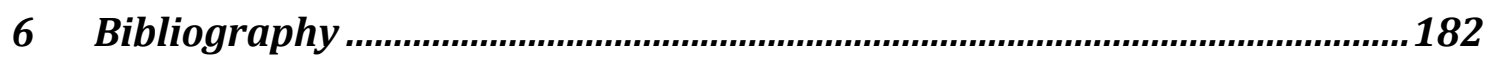

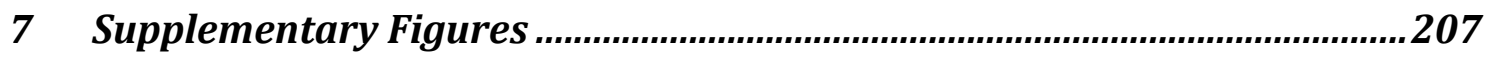

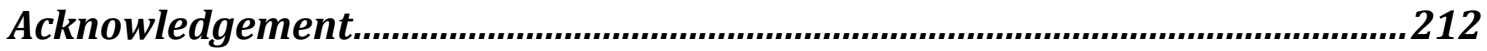




\section{List of Figures}

Figure 2.1: Different subtypes of autophagy (Yen and Klionsky, 2008) ........................ 8

Figure 2.2: Assembly of the Cvt-complex (modified from Reggiori and Klionsky (2013)) 9

Figure 2.3: Structure of the Atg1 kinase complex (modified from Suzuki et al. (2016)) .12

Figure 2.4: Regulation of the Atg1 kinase complex by TORC1 (modified from Noda and Fujioka (2015)). 13

Figure 2.5: Incorporation of the Atg9-containing vesicles into the supra complex of Atg1-Atg13-Atg17-Atg31-Atg29 (modified from Hurley and Young (2017)) ........... 15 Figure 2.6: Composition of the PI3-kinase complexes I and II (modified from Reidick et al. (2017)) .17

Figure 2.7: The two ubiquitin-like conjugation systems in autophagy (modified from Nakatogawa et al. (2009)) .19

Figure 2.8: Structural and sequential requirements for AIM-dependent Atg8 interaction. 21

Figure 2.9: Proposed structure of the coat-like scaffolding complex of Atg8-Atg12Atg5-Atg16 on the surface of the phagophore (Kaufmann and Wollert, 2014)........23 Figure 2.10: Proposed membrane sources for autophagosome formation (Wen and Klionsky, 2016) .24

Figure 2.11: Model for autophagosome maturation and fusion with the vacuole (modified from Reggiori and Ungermann (2017) and Gao et al. (2018b)).................28

Figure 2.12: Structure and model for PI3P binding of KlHsv2 ........................................ 31 Figure 2.13: Model for the organization of the Atg8 lipidation by Atg21 at the

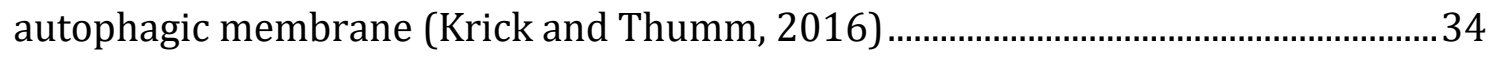
Figure 3.1: Setup of a semidry Western-Blot chamber.....................................................66 Figure 4.1: Overview of the crystal structure of KlAtg21 in complex with AgAtg16 (40-124) .75

Figure 4.2: Sequence alignment of yeast and fungal PROPPINs together with the human orthologue WIPI2 .76 
Figure 4.3: Overview of the potentially interacting amino acids of Atg21 and Atg16 in the crystal structure of KlAtg21 and AgAtg16 (40-124). 78 Figure 4.4: Effect of the Atg21 mutations R151E and K152E on the Cvt-pathway and the interaction with Atg16. 80 Figure 4.5: Simultaneous charge change of Atg21 R151 (R151E) and Atg16 D101 (D101R) partially restores the interaction of both proteins.

Figure 4.6: Simultaneous charge change of Atg21 R151 (R151E) and Atg16 D101 (D101R) restores the function of the Cvt-pathway

Figure 4.7: Simultaneous charge change of Atg21 R151 (R151E) and Atg16 D101 (D101R) restores the localization of Atg16 .85

Figure 4.8: Mutation of other potentially interacting amino acids in Atg21 and Atg16 shows no or only minor effects on the Cvt-pathway.

Figure 4.9: Effect of the mutation of Atg21 D28R, Atg21 K130E or Atg16 K94E on the interaction and localization of Atg21 and Atg16

Figure 4.10: Evaluating the interaction of Atg16 and Atg21 ${ }^{\text {FTTG }}$ using the GFP-TRAP approach

Figure 4.11: In living yeast cells the interaction of Atg16 with Atg21 FTTG or Atg5 was measured using fluorescence cross-correlation spectroscopy (FCCS) 94

Figure 4.12: Examining the interaction of Atg16 with the Atg21 $1^{\text {FTTG }}$ mutant using the split-ubiquitin assay. 96

Figure 4.13: Analyzing the interaction of Atg16 and Atg21 ${ }^{\text {FTTG }}$ using the BioID assay 98

Figure 4.14: Optimization of the experimental setup for the APE1-overexpression assay. 103

Figure 4.15: Depending on the growth conditions, Atg21-YFP shows different localizations at the mCherry-Atg8 positive phagophore 106 Figure 4.16: Localization of Atg3-GFP and its AIM and C234A mutant at the phagophore. 108

Figure 4.17: Atg14 localizes to a dot at the contact site of vacuole and phagophore while PI3P coveres the whole phagophore 111

Figure 4.18: Atg18 is restricted to the rim of the growing phagophore. 113

Figure 4.19: Vac8-GFP is enriched at the contact site between vacuole and phagophore. 116 
Figure 4.20: Vac8-3xGFP is enriched at the contact site of the vacuolar membrane and the phagophore independent of the NVJ 118

Figure 4.21: Deletion of ATG21 and VAC8 significantly reduces the formation of phagophores. 120

Figure 4.22: Upon deletion of VAC8 the contact between the PAS/phagophore and the vacuolar membrane is highly reduced

Figure 4.23: Vac8 precipitates together with GFP-Atg21 using the GFP-TRAP approach 124

Figure 4.24: Vph1-GFP is excluded from the contact site between vacuole and phagophore 127

Figure 4.25: The exclusion of Vph1-GFP from the contact site between vacuole and phagophore is independent of the NVJ. 129

Figure 4.26: Pho8 is not excluded from the contact site between vacuole and phagophore 130

Figure 4.27: FM4-64 is not excluded from the contact site between vacuole and phagophore and there is no flux of FM4-64 to the phagophore 132

Figure 4.28: Atg21 localizes to the contact site between vacuole and phagophore 133

Figure 4.29: The ER is in close proximity to the edges of the growing phagophore but not to the vacuole-phagophore contact site. 135

Figure 4.30: Workflow of the BioID assay (modified from Opitz et al. (2017))..... 137

Figure 4.31: Expression levels and function of the BirA* fusion proteins 139

Figure 4.32: Pattern of the isolated biotinylated proteins from cells carrying BirA*Atg21, Atg21-BirA* or BirA* alone. 142

Figure 4.33: Workflow for the SILAC-based BioID experiments 145

Figure 5.1: Models of the structure of the Atg8 lipidation complex in yeast and mammals. 155

Figure 5.2: Two proposed models of the membrane binding of the Atg21-Atg16 complex.

Figure 7.1: Only the simultaneous charge change of Atg21 R151 (R151E) and Atg16 D101 (D101R) partially restores the interaction of both proteins 207

Figure 7.2: Colocalization of GFP-Atg8 with GAC in the 3xtagBFP-PHO8 expressing $\mathrm{WT}, \operatorname{atg} 21 \Delta$ and vav8 $\Delta$ strains 208 
Figure 7.3: Plasmid maps of the BirA* fusion vectors pUG36-Myc-BirA*-MCS and pUG-MCS-Myc-BirA* 209

Figure 7.4: Pattern of the isolated biotinylated proteins from cells carrying BirA*Atg21, Atg21-BirA* or BirA* alone 210 Figure 7.5: Spatial grouping of the potential interaction partners of Atg21 according to their reported association with distinct compartments in the cell 211 


\section{List of Tables}

Table 3.1: Saccharomyces cerevisiae strains used in this study.........................................

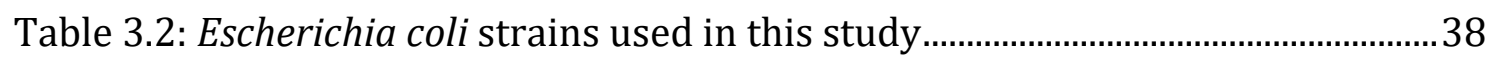

Table 3.3: Plasmids used for molecular cloning and gene expression in this study38 Table 3.4: Plasmids used to amplify selection cassettes for gene deletions and

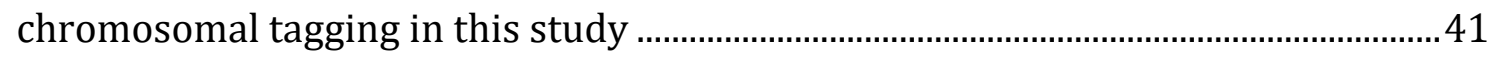

Table 3.5: Oligonucleotides for cloning and sequencing used in this study ................41

Table 3.6: Oligonucleotides for gene deletion and chromosomal tagging used in this study

Table 3.7: Oligonucleotides for site-directed mutagenesis used in this study ..........44

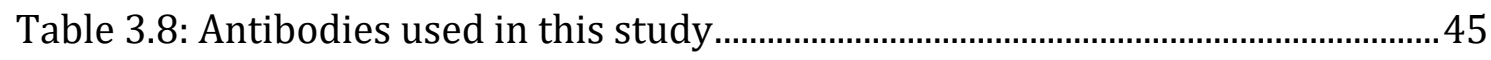

Table 3.9: Commercially available kits and master mixes used in this study............46

Table 3.10: Chemicals, supplements and protein purification systems used in this

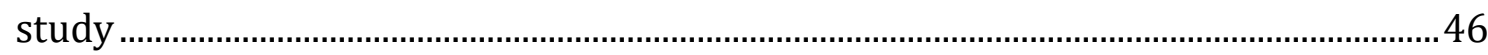

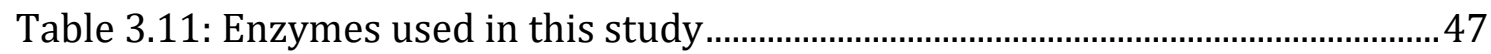

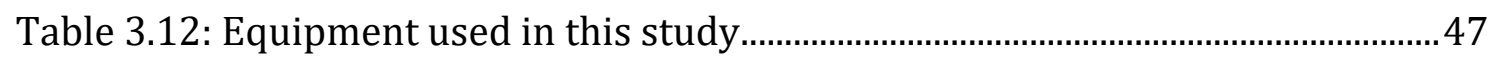

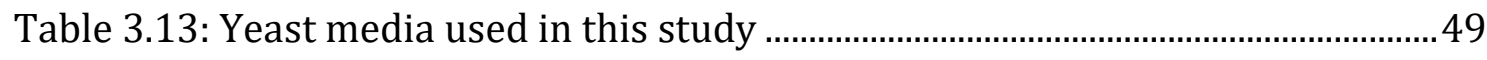

Table 3.14: Composition of the drop-out mix that was used to prepare the CM

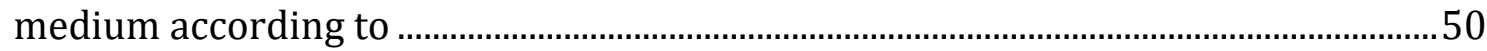

Table 3.15: Supplements for yeast media used in this study .........................................50

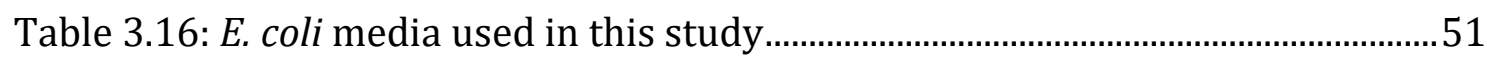

Table 3.17: Composition of regularly used buffers in this study .................................51

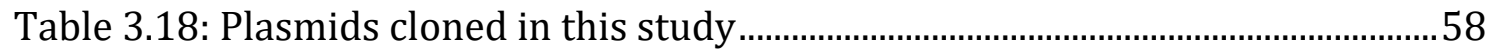

Table 3.19: Overview of the used filter sets for live-cell fluorescence microscopy 62 Table 3.20: Composition of one SDS-polyacrylamide gel for a Mini-PROTEAN ${ }^{\circledR}$ III electrophoresis chamber from Bio-Rad .65

Table 3.21: List of changes in the experimental conditions using the $0.2 \mathrm{ml}$ or the 1 ml Gravity flow Strep-Tactin ${ }^{\circledR}$ Sepharose ${ }^{\circledR}$ Column (iba)................................................71

Table 4.1: List of the identified amino acids potentially contributing to the interaction of Atg21 and Atg16. 77 
Table 5.1: List of the reported interacting regions between PROPPIN-family

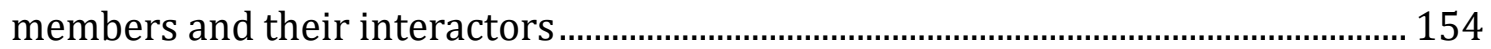




\section{Abbreviation}

2D

3D

5-FOA

AIM

approx.

APS

Atg/ATG

Atg21 FTTG

a.u.

BFP

BioID

BirA*

bp

BSA

$C$

CBB

CCD

CFP

chap.

CM medium

COPII

C-terminus/-

terminal

$\mathrm{Cub}_{\mathrm{ub}}$

Cvt

DAPI

DMSO

DNA

DTT

EDTA

e.g.

ER

ERES

et al.

FCCS

FITC

for

FYVE

$\mathrm{g}$

g

GAC

GDP

GEF

GFP

GTP 2-dimensional

3-dimensional

5-fluoroorotic acid

Atg8-interacting motif

approximately

ammonium persulfate

autophagy-related

Atg21R343T R344T

arbitrary unit

blue fluorescent protein

proximity-dependent biotin identification

BirA ${ }^{\mathrm{R} 118 \mathrm{G}}$

base pair(s)

bovine serum albumin

centi

coomassie brilliant blue

coiled-coil domain

cyan fluorescent protein

Chapter

complete minimal medium

coat protein complex II

carboxy-terminus/ -terminal

C-terminal half of ubiquitin

Cytosol-to-vacuole targeting

4',6-diamidino-2-phenyliindole

dimethyl sulfoxide

deoxyribonucleic acid

dithiothreitol

ethylenediaminetetraacetic acid

exempli gratia (for example)

endoplasmic reticulum

endoplasmic reticulum exit sites

et alii

fluorescence cross-correlation spectroscopy

fluorescein isothiocyanate

forward

Fab1, YOTB, Vac1 and EEA1

gram

gravity

giant pApe1 complex

guanosine diphosphate

guanine nucleotide exchange factor

green fluorescent protein

guanosine triphosphate 


\begin{tabular}{|c|c|}
\hline GTPase & guanosine triphosphatase \\
\hline GUV & giant unilamellar vesicle \\
\hline $\mathrm{h}$ & hour(s) \\
\hline HA & human influenza hemagglutinin \\
\hline HEPES & 4-(2-hydroxyethyl)-1-piperazineethanesulfonic acid \\
\hline His & histidine \\
\hline HORMA domain & Hop1, Rev7 and Mad2 domain \\
\hline HOPS & homotypic vacuole fusion and protein sorting \\
\hline HRP & horseradish peroxidase \\
\hline IDR & intrinsic disordered region \\
\hline $\mathrm{kb}$ & kilobases \\
\hline $\mathrm{kDa}$ & kilodalton \\
\hline 1 & liter \\
\hline LB & lysogeny broth \\
\hline LC-MS & liquid chromatography-mass spectrometry \\
\hline $\mathrm{Ld}$ & liquid disordered \\
\hline Lo & liquid ordered \\
\hline M & molar \\
\hline $\mathrm{m}$ & meter \\
\hline $\mathrm{m}$ & milli \\
\hline mApe1 & mature aminopeptidase 1 \\
\hline mCherry & monomeric Cherry \\
\hline MCS & multiple cloning site \\
\hline MIM & MIT-interacting motif \\
\hline $\min$ & minute(s) \\
\hline MIT domain & microtubule-interacting and transport domain \\
\hline MS & mass spectrometry \\
\hline MV & minimal plus vitamins \\
\hline $\mathrm{n}$ & nano \\
\hline $\begin{array}{l}\mathrm{N} \text {-terminus/- } \\
\text { terminal }\end{array}$ & amino-terminus / -terminal \\
\hline $\mathrm{N}_{\mathrm{ub}}$ & $\mathrm{N}$-terminal half of ubiquitin \\
\hline NVJ & nucleus-vacuole junction \\
\hline ns & not significant \\
\hline OD 600 & optical density at a wavelength of $600 \mathrm{~nm}$ \\
\hline pApe1 & precursor aminopeptidase 1 \\
\hline PAS & pre-autophagosomal structure \\
\hline PBS & phosphate buffered saline \\
\hline PCR & polymerase chain reaction \\
\hline PDB & protein data bank \\
\hline PE & phosphatidylethanolamine \\
\hline PEG & polyethylene glycol \\
\hline $\mathrm{pH}$ & potential of hydrogen \\
\hline PI & phosphatidylinositol \\
\hline PI3-kinase & phosphatidylinositol 3-kinase \\
\hline PI3P & phosphatidylinositol 3-phosphate \\
\hline $\mathrm{PI} 3,5 \mathrm{P}_{2}$ & phosphatidylinositol 3,5-bisphosphate \\
\hline PKA & protein kinase $A$ \\
\hline PMN & piecemeal microautophagy of the nucleus \\
\hline
\end{tabular}




\begin{tabular}{ll} 
PMSF & phenylmethane sulfonyl fluoride \\
PROPPIN & $\beta$-propellers that bind polyphosphoinositides \\
PSM & peptide spectrum matches \\
PVDF & polyvinylidene difluoride \\
rev & reverse \\
RFP & red fluorescent protein \\
rpm & rounds per minute \\
RT & room temperature \\
SD-N & synthetic defined medium without nitrogen \\
SDS & sodium dodecyl sulfate \\
SDS-PAGE & sodium dodecyl sulfate polyacrylamide gel electrophoresis \\
sec & second(s) \\
SEM & standard error of the mean \\
SILAC & stable isotope labeling by amino acids \\
SNARE & soluble N-ethylmaleimide-sensitive-factor attachment \\
SOC & receptor \\
TAE & super optimal broth with catabolite repression \\
TBST & tris base, acetic acid, EDTA \\
TCA & tris buffered saline with triton X-100 \\
TEMED & trichloroacetic acid \\
Tris & tetramethylethylendiamin \\
TRITC & tris(hydroxymethyl)aminomethane \\
Trp & tetramethylrhodamine \\
TORC1 & tryptophan \\
Ura & target of rapamycin complex 1 \\
UV & uracil \\
v/v & ultra violet \\
WIPI & volume per volume \\
w/o & WD40 repeat-containing proteins that interact with \\
WT & phosphoinositides \\
w/v & without \\
YPD & wild type \\
$\AA$ & weight per volume \\
$\mu$ & yeast peptone dextrose \\
& angstrom \\
\hline & micro \\
\hline
\end{tabular}




\section{Summary}

Macroautophagy is a degradative membrane trafficking pathway that removes and recycles cellular components to maintain cellular homeostasis. Targets for degradation are a variety of cytosolic components including protein aggregates, damaged or superfluous organelles or invading pathogens. The transport process can either be unselective or selective but always involves the sequestration of a cargo by a double-membraned vesicle, the autophagosome. Upon starvation the autophagosome biogenesis is initiated at the pre-autophagosomal structure (PAS) where a cup-shaped membrane structure is assembled de novo (phagophore) to engulf cytosolic material. After closure, the autophagosome fuses with the vacuole to release its cargo to the vacuolar lumen for degradation and reuse.

A crucial factor for autophagosome biogenesis is the generation of phosphatidylinositol 3-phosphate (PI3P) at the PAS. The presence of PI3P on autophagic membranes is deciphered by $\beta$-propellers that bind polyphosphoinositides (PROPPIN). PROPPINs are a highly conserved family of WD40-repeat proteins which fold as seven-bladed $\beta$-propellers. PI3P binding is mediated by a conserved FRRG-motif that participates in the formation of two lipidbinding sites at the circumference of the propeller.

Another important step required for the elongation of the phagophore, is the conjugation of ubiquitin-like Atg8 to the lipid phosphatidylethanolamine (PE) which is mediated by two ubiquitin-like conjugation systems. The first system results in the coupling of ubiquitin-like Atg12 to Atg5. In complex with Atg16, the Atg12 Atg5 conjugate acts as an E3-like enzyme to facilitate the transfer of Atg8 from its E2-like enzyme Atg3 to PE.

This study focused on the investigation of the molecular functions of the S. cerevisiae PROPPIN Atg21. So far, the only known function of Atg21 is to organize the lipidation of Atg8 at the PI3P-positive autophagic membrane. It recruits the Atg8 Atg3 conjugate and the Atg12 Atg5/Atg16 complex to the PAS by a direct interaction with Atg8 and Atg16. Unlike its homolog Atg18, Atg21 is not essential for bulk autophagy but is indispensable for some selective types of autophagy including the Cytosol-to-vacuole targeting (Cvt) pathway. The Cvt-pathway 
selectively transports proaminopeptidase 1 (pApe1) and other hydrolases to the vacuole under nutrient rich conditions.

In parallel to this study the cooperation partners Dr. Karin Kühnel and Dr. Janina Metje were able to solve the crystal structure of Kluyveromyces lactis Atg21 in complex with the coiled-coil domain of Ashbya gossypii Atg16 up to a resolution of $4 \AA$. This is the first crystal structure of a PROPPIN-family member in complex with its interactor. On the basis of the crystal structure, a detailed analysis of the interacting domains of Atg21 and Atg16 was performed in this study. A variety of in vivo and in vitro experiments revealed that Atg21 and Atg16 interact through the formation of a single salt bridge between Atg21 R151 and Atg16 D101. This salt bridge is essential for the proper targeting of the Atg12 Atg5/Atg16 complex to the PAS as well as for an efficient progression of the Cvt-pathway. Surrounding amino acids seem to stabilize the salt bridge by contributing to the net charge on the surface of both proteins but are dispensable for their interaction and function. This interaction mechanism of Atg21 and Atg16 is conserved from yeast to mammals.

Fluorescence cross-correlation spectroscopy (FCCS) in combination with other approaches indicated that the Atg21-Atg16 complex might be preferentially assembled at the autophagic membrane and not in the cytosol.

Overexpression of APE1, the cargo of the Cvt-pathway, results into the formation of an artificially enlarged cargo complex which enables the monitoring of phagophore assembly and elongation by fluorescence microscopy (APE1-overexpression assay). In this study, using the APE1-overexpression assay, a yet unknown contact site between the vacuole and the phagophore was identified. The contact site is established by the vacuolar protein Vac8 and is important for the elongation of the phagophore. Although Vac8 seems to be not essential for the assembly of the PAS, it defines the normal site of its formation. The vacuole-phagophore contact site coincides with the formation of a specialized membrane domain at the vacuolar membrane. This membrane domain is characterized by the enrichment of Vac8 and the exclusion of the membrane integral protein Vph1. This suggests the formation of a lipid raft at the vacuolar membrane although the contribution of lipids to the membrane domain requires further investigation. 
Under starvation conditions the localization of Atg21 at the phagophore is restricted to the contact site between vacuole and phagophore. Thereby, Atg21 targets the Atg8 lipidation to the highly curved rim of the growing phagophore. Atg21 is not essential for the formation of the vacuole-phagophore contact site. Nevertheless, it might contribute to it as a potential interactor of Vac8.

Similar to Atg21, its homolog Atg18 also resides on the rim of the phagophore although PI3P is dispersed over the whole phagophore. Therefore, there must be additional mechanisms that regulate the localization of PROPPINs at the phagophore. This could include the sensing of membrane curvature or even the induction of membrane bending.

PROPPINs localize to various organelles in the cell (endosomes, vacuole and autophagic membrane) suggesting that they exhibit a variety of functions. Nevertheless, very little is known about their other functions. In this study, the proximity dependent biotin identification (BioID) assay in combination with a stable isotope labeling by amino acids (SILAC) based approach was used to quantitatively identify potential interactors of Atg21. In an initial experiment 17 potential interaction partners of Atg21 were identified. The majority of the identified proteins can be characterized as membrane associated proteins which reside on vacuolar and endosomal membranes or on cytosolic vesicles. Functionally most of them participate in transport processes, mainly vesicle-mediated transport processes. The localization and function of the potential interactors of Atg21 highly correlates with the localization of Atg21. Therefore, the 17 potential interaction partners of Atg21 provide a promising basis for the elucidation of the role of Atg21 in the cell. 


\section{Introduction}

\subsection{Autophagy}

The term autophagy derives from the Greek words auto (self) and phagein (to eat) and describes a regulated degradative process of self-digestion. This evolutionarily conserved highly organized membrane-trafficking pathway is used to maintain cellular homeostasis by the degradation and recycling of cellular components. Here, macromolecules and organelles are transported via vesicles from the cytosol to the lytic compartment, which is the lysosome in higher eukaryotes or the vacuole in fungi and plants. From lysis and degradation of these no longer needed components, the cell is able to rapidly gain energy as well as building blocks to renew cellular components. It can, therefore, adapt to various stresses such as nutrient deprivation (Reggiori and Klionsky, 2013).

However, autophagy is much more than a simple stress response mechanism. It plays a major role in many essential cellular functions including the quality control of proteins and organelles, organellar remodeling, destruction of pathogens (e.g. bacteria), cell differentiation as well as the regulation of immunity and inflammation. Due to its diverse roles in the cell, it is not surprising that autophagic malfunction was found to be associated with a range of illnesses like neurodegenerative diseases (Parkinson's and Huntington's disease), diabetes (type 2) and cancer (Mizushima et al., 2008; Reggiori and Klionsky, 2013).

Historically, autophagy was first discovered in the late 1950s and early 1960s. Using transmission electron microscopy, membrane-bound "dense bodies" were observed in mammalian cells which contained semi-digested organelles, like mitochondria, endoplasmic reticulum (ER) membranes or ribosomes (Clark, 1957; Novikoff, 1959; Ashford and Porter, 1962; Novikoff and Essner, 1962). This was the first step to identify autophagy as a regulated process to degrade and recycle cellular components. Later, it was shown that autophagy was induced upon glucagon treatment or starvation while treatment of cells with insulin and addition of amino acids to the medium decreased it (Ashford and Porter, 1962; Novikoff and Essner, 1962; Pfeifer, 1977; Mortimore and Schworer, 1977). This indicated that autophagy 
responds to nutrient deprivation and can be regulated by hormones. Furthermore, it implied a crucial role of autophagy in the catabolic process by recycling nutrients upon external stress factors.

In the 1990s, autophagy was first described to exist in the yeast Saccharomyces cerevisiae (S. cerevisiae) setting the stage for the identification of the first autophagyrelated $(A T G)$ genes using genetic screens (Takeshige et al., 1992; Tsukada and Ohsumi, 1993; Thumm et al., 1994; Harding et al., 1995). This provided the initial breakthrough that caused an intense research on the molecular mechanisms of autophagy, its physiological connection to human illnesses and the development of drugs to target autophagy in various diseases. In 2016, the Noble Prize in Physiology or Medicine was awarded to the Japanese cell biologist Yoshinori Ohsumi for his work on the mechanisms of autophagy.

Autophagy can be classified into the two main types, microautophagy and macroautophagy, which can be further subdivided into nonselective (e.g. under starvation) and selective processes (Figure 2.1; Reggiori and Klionsky, 2013). In mammalian cells, a third non-vesicular type of autophagy was described which was termed chaperone-mediated autophagy (Cuervo and Wong, 2014). The autophagy model system $S$. cerevisiae as well as the underlying molecular mechanisms of the autophagic subtypes are detailed in the following chapters.

\subsubsection{The yeast S. cerevisiae as a model organism to study autophagy}

The first autophagic genes were discovered in the yeast $S$. cerevisiae which set the stage for the investigation of the molecular mechanisms of autophagy (Tsukada and Ohsumi, 1993; Thumm et al., 1994; Harding et al., 1995). Until now, autophagy has been best characterized in the model organism $S$. cerevisiae. To this date, $42 \mathrm{Atg}$ proteins have been identified in S. cerevisiae that function in any kind of autophagy (Wen and Klionsky, 2016; Parzych et al., 2018).

The budding yeast $S$. cerevisiae is a unicellular eukaryotic organism with a round to oval shape and a size of 5-10 $\mu \mathrm{m}$ in diameter. It was the first eukaryotic organism whose genome was fully sequenced (Goffeau et al., 1996). A normal haploid cell harbors about $12000 \mathrm{~kb}$ of genomic DNA which is distributed on 16 chromosomes. The genome was predicted to comprise about 6000 open reading frames of which 
approx. 80\% have been verified (Goffeau et al., 1996; Duina et al., 2014). S. cerevisiae can be grown in a haploid or diploid form and has a simple life cycle. Both forms (haploid and diploid) replicate by mitotic division with a doubling time of $90 \mathrm{~min}$ under ideal nutrient conditions. Nutrient shortage induces sporulation of the diploid cells, characterized by meiotic division and the formation of an ascus which contains four haploid spores. The four spores are equally divided into the two mating types MATa and MAT $\alpha$. Two haploid cells of different mating types can mate to form a diploid cell (Neiman, 2005).

S. cerevisiae is frequently used as a model for human cells due to a variety of advantages. It can be easily and inexpensively cultured under a variety of stress conditions and has a short generation time. It is technically easy to grow large amounts for the purification of native protein complexes in high quantities (e.g. for structural analysis) (Duina et al., 2014). Furthermore, efficient tools have been developed that allow for a precise genetic manipulation by homologous recombination, enabling the deletion and tagging of open reading frames (Longtine et al., 1998; Knop et al., 1999; Janke et al., 2004). Moreover, the plasmid-based expression of genes is well established. This allows the effective regulation of the expression level of genes of interest using inducible promoters or high and low copy plasmids (Sikorski and Hieter, 1989; Rose and Broach, 1990; Partow et al., 2010).

Apart from these technical and methodical advantages, S. cerevisiae harbors a special biosynthetic form of selective autophagy, the Cytosol-to-vacuole targeting (Cvt) pathway. It transports degradative enzymes to their place of activity, the vacuolar lumen. The Cvt-pathway is constitutively active under nutrient rich conditions and can therefore be studied independent of the unselective macroautophagy making it an ideal model to study the mechanism of selective autophagy (chap. 2.1.3). Under autophagy inducing conditions the transport of the degradative enzymes is taken over by macroautophagy allowing a separated analysis of both processes (Klionsky et al., 1992; Baba et al., 1997).

Parts of the autophagic machinery exist in the genome of all eukaryotic organisms that have been analyzed, validating its evolutionary conservation. Therefore, the knowledge of the molecular mechanisms of autophagy obtained from S. cerevisiae is 
transferable to higher eukaryotes and can also be relevant for studying human diseases.

\subsubsection{Macroautophagy}

Macroautophagy, hereafter autophagy, in yeast involves the sequestration of bulk cellular material such as damaged organelles and protein aggregates by a de novo forming cup-shaped membrane structure, called the isolation membrane or phagophore (Figure 2.1). The phagophore expands and encloses to fully engulf the cytosolic cargo thereby forming a double-membraned vesicle, the so-called autophagosome. The engulfed cargo is transported to the vacuole for degradation and recycling of its building blocks. Therefore, the outer membrane of the autophagosome fuses with the vacuole releasing the autophagic body, consisting of the cargo enclosed by the inner autophagic membrane, to the lumen of the vacuole. Within the vacuole, the autophagic body is lysed to make its content accessible for vacuolar hydrolases (Reggiori and Klionsky, 2013). Autophagosomes are formed and completed within a time frame of 4-5 min. A whole cycle from the initiation to the fusion with the vacuole was found to last approx. 10 min (Geng et al., 2008; Xie et al., 2008). Closed autophagosomes in the cytosol and autophagic bodies in the vacuolar lumen were found to have a diameter of 400-900 nm (Takeshige et al., 1992; Baba et al., 1994).

Initially, macroautophagy was defined as the unselective degradation of bulk cytosolic material and organelles. In addition, a subset of macroautophagic processes are known which selectively degrade specific cargos. Most of these processes use the autophagic core machinery and morphologically remind of unselective autophagy. Crucial factors of selective autophagy are selective cargo receptors which mark the cargo for degradation. They initiate the formation of a phagophore by the interaction with the autophagic core machinery (Farré and Subramani, 2016). The sequestering membrane is tightly formed around the cargo excluding other cytosolic material (Reggiori and Klionsky, 2013). Specific cargos can be for example damaged or superfluous mitochondria (mitophagy), peroxisomes (pexophagy), ER (ER-phagy) or ribosomes (ribophagy) (Farré et al., 2008; Kraft et al., 2008; Mochida et al., 2015; Kanki et al., 2015). In mammalian cells, further selective mechanisms exist that include the degradation of pathogens like bacteria 
(xenophagy). Depending on the form, selective autophagy can occur under normal growth conditions (e.g. the Cvt-pathway) or can be induced by different stimuli like oxidative, osmotic or hypoxic stress as well as starvation (Farré and Subramani, 2016).

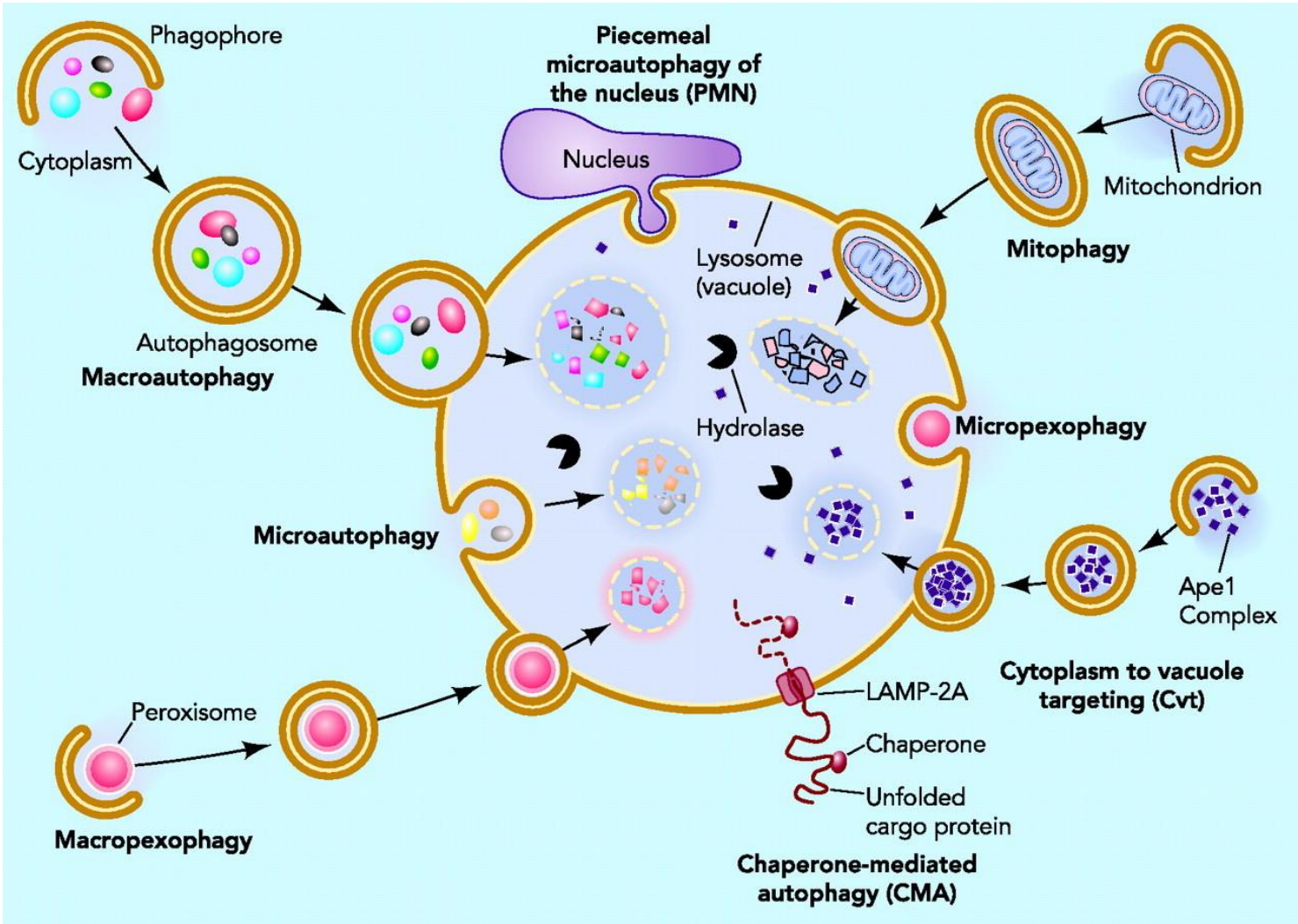

Figure 2.1: Different subtypes of autophagy (Yen and Klionsky, 2008)

Autophagy can be classified into three main types macroautophagy, microautophagy and chaperonemediated autophagy. Macroautophagy involves the formation of a double-membraned cup-like structure (phagophore) that engulfs cytosolic material. Upon closure the so-called autophagosome fuses with the lysosome/vacuole to release its content to the lumen for degradation. The process can be selective for specific organelles or protein complexes such as mitochondria, peroxisomes or the Ape1-complex. During microautophagy the cargo is directly engulfed by the lysosomal/vacuolar membrane. Selective forms of microautophagy are for example micropexophagy or piecemeal microautophagy of the nucleus where peroxisomes or parts of the nucleus are degraded respectively. Chaperone-mediated autophagy directly translocates unfolded proteins across the vacuolar membrane in a chaperone-dependent manner. So far, it was only found to be present in mammalian cells.

\subsubsection{The Cytosol-to-vacuole targeting pathway as a model of selective autophagy}

The Cvt-pathway is a process that constitutively and selectively transports vacuolar hydrolases to the vacuole. The core cargo of the Cvt-pathway is the soluble leucine aminopeptidase 1 (Ape1). Ape 1 is first synthesized in the cytosol as an inactive precursor form (pApe1) which contains a N-terminal 45 amino acid-long propeptide 
(Klionsky et al., 1992). After synthesis, pApe1 directly oligomerizes to form a homododecamer (Kim et al., 1997). Mediated by the propeptide the homododecamers of pApe1 further aggregate into a larger oligomeric structure to form the so-called Ape1-complex (Figure 2.2). Accessible propeptides on the surface of the Ape1-complex are recognized by the specific cargo receptor Atg19 (Leber et al., 2001; Scott et al., 2001; Shintani et al., 2002). Apart from pApe1, Atg19 recruits additional cargo proteins including the $\alpha$-mannosidase Ams1 and the aspartyl aminopeptidase Ape4 to the Ape1-complex, thereby forming the Cvt-complex (Hutchins and Klionsky, 2001; Yuga et al., 2011). Furthermore, the retrotransposon Ty1 and the leucine aminopeptidase III (Lap3) were reported to be transported to the vacuole in an Atg19-dependent manner. However, the transport of Lap3 to the vacuole was only observed under nutrient deprivation. Therefore, it might not be a selective cargo of the Cvt-pathway (Kageyama et al., 2009; Suzuki et al., 2011).

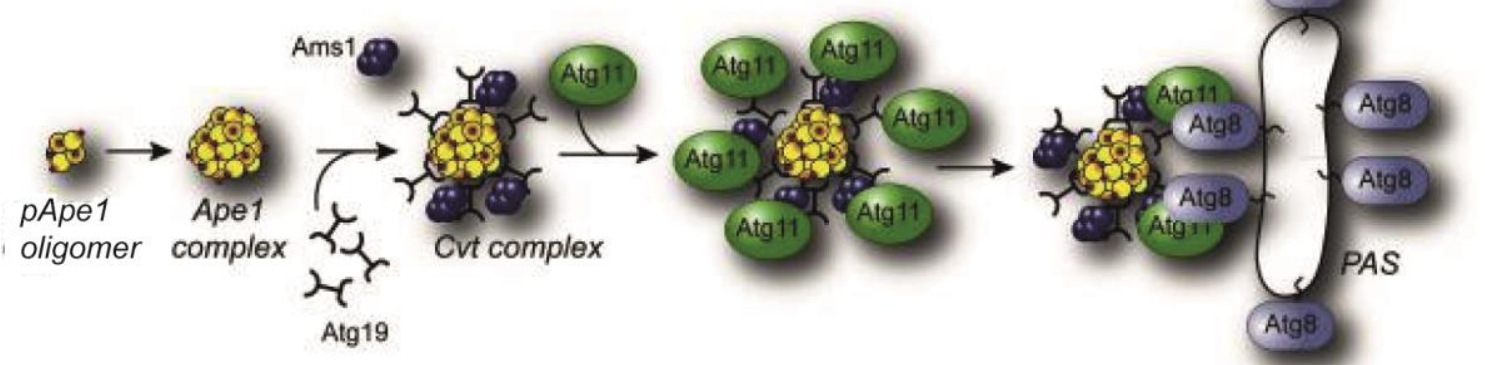

Figure 2.2: Assembly of the Cvt-complex (modified from Reggiori and Klionsky (2013))

The inactive precursor form of Ape1 (pApe1) is synthesized in the cytosol where it forms homododecamers. These dodecamers self-assemble into a larger oligomeric structure, called Ape1-complex. Interaction of Atg19 with the Ape1-complex and further recruitment of Ams1 forms the Cvt-complex. Atg11 binds to the Cvt-complex in an Atg19-dependent manner and initiates the formation of a sequestering membrane by recruitment of the autophagic core machinery.

Through the interaction with Atg19, Atg11 and Atg8 bind to the Cvt-complex and subsequently recruit the autophagic core machinery to induce the formation of a sequestering membrane (Shintani et al., 2002; Noda et al., 2010). Excluding other cellular material, the Cvt-complex is completely engulfed by the membrane forming the so-called Cvt-vesicle. With a diameter of 140-160 nm, the Cvt-vesicles are much smaller than autophagosomes (Baba et al., 1997). Similar to autophagosomes, Cvt-vesicles fuse with the vacuole to release their cargo into the vacuolar lumen. In the vacuole, the $\mathrm{N}$-terminal propeptide of pApe1 is cleaved off in a proteinase $\mathrm{A}$ (Pep4)-dependent manner resulting in its mature and active form (mApe1) 
(Klionsky et al., 1992). Under starvation conditions, the transport of the Cvtcomplex is taken over by autophagy. The molecular weight shift of about $10 \mathrm{kDa}$ between pApe1 and mApe1 is detectable by Western-Blot analysis and therefore often used as a readout for the progression of the Cvt-pathway (nutrient rich conditions) or unselective bulk autophagy (starvation) (Klionsky et al., 1992; Baba et al., 1997).

\subsubsection{Microautophagy}

Microautophagy is defined as a type of autophagy where a cargo is directly internalized by the vacuole. To this end, the vacuolar membrane invaginates to enclose the cargo (Figure 2.1). The forming vesicle buds off into the vacuolar lumen where it is subsequently degraded by vacuolar hydrolases. Nonselective microautophagy is a constitutive process but can be enhanced by starvation or rapamycin (Dubouloz et al., 2005; Li et al., 2012). Several forms of microautophagy were found to require components of the macroautophagic machinery, such as the two ubiquitin-like conjugation systems (chap. 2.2.4). Selective forms of microautophagy are for example micropexophagy, micromitophagy and piecemeal microautophagy of the nucleus (PMN). In addition, lipid droplets were found to be selectively degraded in a microautophagic process as well (Roberts et al., 2003; Farré and Subramani, 2004; Li et al., 2012; Graef, 2018).

\subsubsection{Chaperone-mediated autophagy}

The third main type of autophagy is chaperone-mediated autophagy which is only present in mammalian cells. In contrast to macro- and microautophagy, the transport of the cargo to the lysosomal lumen via chaperone-mediated autophagy does not require vesicles or membrane invaginations. Instead, the cargo proteins are directly transported across the membrane via a protein translocation process (Figure 2.1). Cargo proteins harboring a KFERQ-like motif are recognized in the cytosol by the chaperon hsc70 (heat shock-cognate protein of $\underline{70} \mathrm{kDa}$ ). hsc70 targets the bound proteins to the lysosomal membrane where they interact with the transmembrane protein LAMP2A (lysosome-associated membrane protein type $\underline{2 A})$. Upon interaction with the cargo protein, LAMP2A oligomerizes to form the translocation complex. Mediated by hsc70 and other chaperones the cargo protein 
is unfolded before its translocation to the lysosomal lumen. The transport across the membrane is dependent on the lysosomal hsc70. Translocated proteins are degraded by lysosomal hydrolases. Chaperone-mediated autophagy contributes to the amino acid recycling under prolonged nutrient deprivation and is a quality control mechanism for cytosolic proteins (Cuervo and Wong, 2014).

\subsection{Induction of autophagy and the autophagic core machinery}

Phagophore formation is initiated at a perivacuolar site which is called the preautophagosomal structure (PAS). Most of the Atg proteins are at least transiently associated with the PAS during autophagosome formation (Suzuki et al., 2001; Suzuki et al., 2007). Therefore, it can be defined as a dynamic precursor structure that induces the nucleation of the autophagic membrane. Of the 42 known Atg proteins in yeast, 18 are essential for the formation of autophagosomes. They are recruited to the PAS in a hierarchical manner and can be functionally grouped into the Atg1 kinase complex, Atg9-containing vesicles, the phosphatidylinositol 3kinase (PI3-kinase) complex and the two ubiquitin-like conjugation systems (Suzuki et al., 2007; Mizushima et al., 2011). These functional groups are required for all autophagic subtypes and are therefore referred to as the core autophagic machinery. Furthermore, they are evolutionary conserved from yeast to mammals (Wen and Klionsky, 2016). The structures and molecular functions of the autophagic core machinery are outlined in the following chapters. Since the budding yeast S. cerevisiae was the model organism used in this study, the following chapters mainly detail the structure and molecular function of the autophagic core machinery in yeast.

\subsubsection{The Atg1 kinase complex and autophagy induction}

The Atg1 kinase complex, also named the autophagy initiation complex, acts most upstream in the autophagic machinery and is a core component of the PAS. It is thought to function as a platform to receive autophagy inducing signals (Suzuki et al., 2007; Cheong et al., 2008; Kawamata et al., 2008).

The yeast Atg1 kinase complex is comprised of Atg1, the only Ser/Thr kinase among the autophagic core proteins, Atg13 a regulatory subunit as well as the subcomplex 
of Atg17-Atg31-Atg29 which has in part a scaffolding function (Figure 2.3 A). In most selective forms of autophagy, the scaffolding function of the Atg17-Atg31Atg29 complex can be taken over by Atg11 which links the cargo receptor to the autophagic core machinery (Kabeya et al., 2007; Kawamata et al., 2008; Farré and Subramani, 2016).
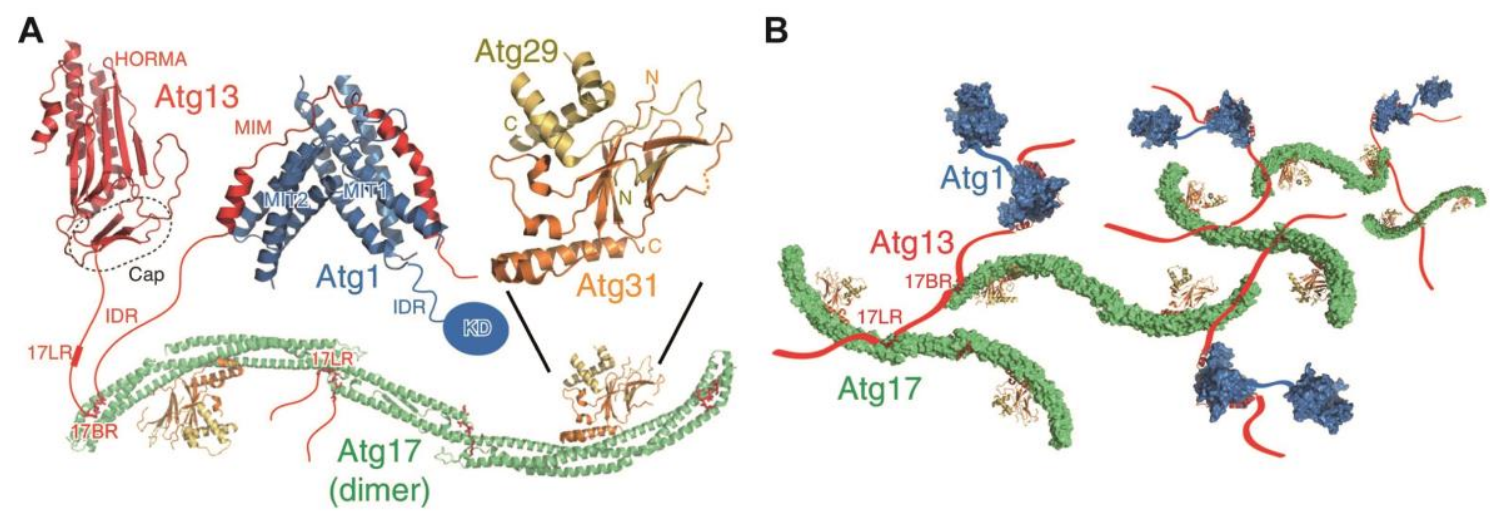

Figure 2.3: Structure of the Atg1 kinase complex (modified from Suzuki et al. (2016))

(A) Cartoon representation of the crystal structures of each of the single components of the Atg1 kinase complex. Atg1 (blue) comprises a N-terminal kinase domain (KD) connected to two C-terminal tandem microtubule-interacting and transport (MIT1 and 2) domains via an intrinsically disordered region (IDR). Atg13 (red) contains a N-terminal Hop1, Rev7 and Mad2 (HORMA) domain linked to a C-terminal IDR. The IDR contains two Atg17-binding regions (17LR and 17BR) and a helical region called the MIT-interacting motif (MIM) which mediates the binding to the MIT domains of Atg1. The homodimer of Atg17 (green) displays a S-shaped architecture. The subcomplex of Atg31 (orange) and Atg29 (yellow) binds to the concave side of Atg17. (B) Atg13 connects multiple Atg17 dimers using 17LR and 17BR. Thereby, a supra oligomeric structure of the Atg1 kinase complex is assembled.

The Ser/Thr kinase Atg1 consists of a N-terminal kinase domain (KD) that is connected to two C-terminal tandem microtubule-interacting and transport (MIT) domains via an intrinsically disordered region (IDR). The two MIT domains are required for the binding of Atg13 (Fujioka et al., 2014). The regulatory protein Atg13 contains a N-terminal Hop1, Rev7 and Mad2 (HORMA) domain linked to a C-terminal IDR. The IDR harbors a helical region called the MIT-interacting motif (MIM) which mediates the binding to the MIT domains of Atg1. Furthermore, the IDR comprises two functionally relevant Atg17-binding regions (17LR and 17BR) which link Atg17 dimers to each other (Jao et al., 2013; Fujioka et al., 2014; Yamamoto et al., 2016). Atg17 comprises four $\alpha$-helices which fold into a stable crescent-like structure. It self-assembles, forming a homodimer with a S-shaped architecture (Figure $2.3 \mathrm{~A}$ ). The heterodimer of Atg31 and Atg29 interacts with the C-terminal $\alpha$-helix of Atg17 located at the concave side of the crescent-like structure (Kabeya et al., 2009; Ragusa et al., 2012; Mao et al., 2013; Chew et al., 2013). 
The two kinases target of rapamycin complex 1 (TORC1) and the cAMP-dependent protein kinase A (PKA) are two major sensors of nutrient availability which regulate autophagy induction. While TORC1 primarily senses the status of nitrogen sources and amino acids, PKA is thought to be a sensor for glucose (Noda and Ohsumi, 1998; Budovskaya et al., 2004).

Under nutrient rich conditions, TORC1 is active and downregulates autophagy directly through the phosphorylation of multiple sites in its substrate Atg13 (Figure 2.4 A). The hyperphosphorylation of the serine rich Atg13 weakens or blocks its interaction with Atg1 and Atg17 resulting in a low concentration of formed Atg1 kinase complexes (Kamada et al., 2000; Kabeya et al., 2005; Kamada et al., 2010). PKA was identified to be a phosphoregulator of Atg13 and Atg1 as well. It is active at high glucose levels and phosphorylates Atg13 and Atg1 similar to TORC1. However, the phosphorylation sites PKA uses in Atg13 are distinct from those of TORC1. Nevertheless, phosphorylation of those sites by PKA also results in the inhibition of autophagy (Budovskaya et al., 2005; Stephan et al., 2009).

\section{A Nutrient rich}

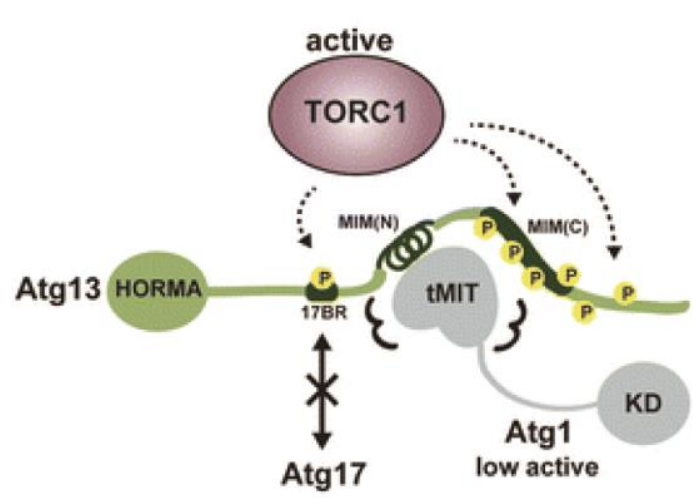

B Starvation

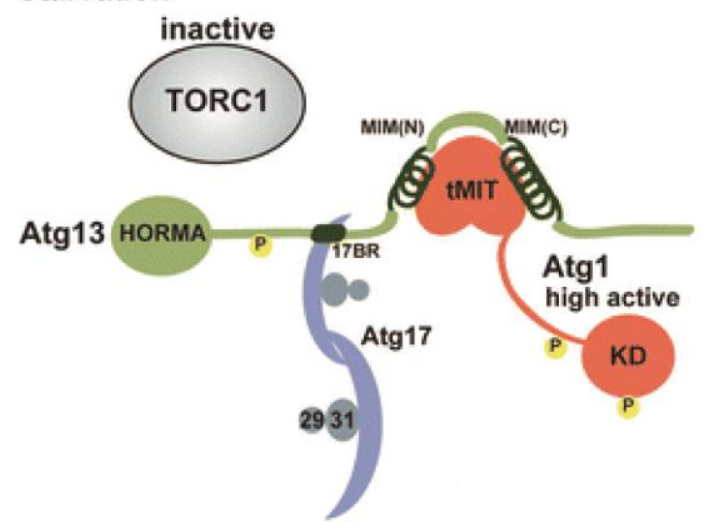

Figure 2.4: Regulation of the Atg1 kinase complex by TORC1 (modified from Noda and Fujioka (2015))

(A) The protein kinase TORC1 is a sensor for nitrogen availability. In its active state under nutrient rich conditions, it directly phosphorylates Atg13 at multiple sites within the Atg17-binding region (17BR) and the C-terminal MIT-interacting motif (MIM(C)). As a result, the interaction with Atg17 is blocked and the interaction with the two C-terminal tandem microtubule-interacting and transport domains (tMIT1) of Atg1 is weakened. This results in low activity of the Atg1 kinase domain (KD). Furthermore, the formation of the Atg1 kinase complex is inhibited. (B) Upon starvation, TORC1 is inactivated resulting in a rapid dephosphorylation of Atg13. This enables the formation of the Atg1 kinase complex.

Upon starvation, TORC1 and PKA are inactivated leading to a rapid dephosphorylation of Atg13 and enabling a high affinity interaction with Atg1 and Atg17 (Figure 2.4 B). Due to its two Atg17-binding regions, Atg13 facilitates the 
molecular bridging of several Atg17-Atg31-Atg29 hexamers resulting in an oligomeric structure of about 50 complexes (Figure 2.3 B; Kabeya et al., 2005; Fujioka et al., 2014; Yamamoto et al., 2016). Incorporation of Atg1 into this oligomeric structure is suggested to promote self-association of Atg1 as well as autophosphorylation which enhances its kinase activity (Yamamoto et al., 2016). The kinase activity of Atg1 is also essential for autophagy initiation although it is not required to target proteins to the PAS (Kamada et al., 2000; Cheong et al., 2008; Sekito et al., 2009). Apart from autophosphorylation, the proteins Atg9 and Atg2 have been identified as substrates of Atg1 (Papinski et al., 2014).

In contrast to yeast, in the mammalian system the Atg1 homolog ULK1 forms a stable complex with Atg13 (Hosokawa et al., 2009). Regardless of the interaction mechanism of Atg1 and Atg13, the resulting Atg1 kinase supra complex forms the core of the PAS and serves as a scaffold to recruit further functional groups of the autophagic core machinery (Suzuki et al., 2007; Kofinger et al., 2015).

\subsubsection{Atg9-containing vesicles}

After the assembly of the Atg1 kinase complex, one of the first components recruited to the PAS are Atg9-containing vesicles. Atg9 is the sole transmembrane protein among the autophagic core proteins. It comprises six predicted membranespanning helices in its central region as well as a large cytosolic IDR on both the Nand the C-terminus (Noda et al., 2000; Lang et al., 2000; He et al., 2008). Atg9 is incorporated into small single-membraned vesicles with a diameter of 30-60 nm, called the Atg9-containing vesicles (Mari et al., 2010; Yamamoto et al., 2012). Within the membrane of these vesicles, Atg9 self-associates forming higher-order oligomeric structures. Oligomerization of Atg9 facilitates its transport to the PAS (He et al., 2008).

Atg9-containing vesicles derive from the trans-Golgi network and are reported to either cluster in a cytosolic reservoir in proximity to mitochondria or to remain highly mobile cytosolic vesicles (Reggiori et al., 2005; Sekito et al., 2009; Mari et al., 2010; Ohashi and Munro, 2010). Independent of the nature of the Atg9 reservoir, Atg9-containing vesicles translocate from their peripheral pool to the PAS upon autophagy induction. The proper transport of Atg9 to the PAS is dependent on the 
peripheral membrane protein Atg23 and the integral membrane protein Atg27 (Yen et al., 2007; Backues et al., 2015). Recently, Atg41 was identified to interact with Atg9 and was suggested to also participate in the delivery of Atg9-containing vesicles to the PAS (Yao et al., 2015).

At the PAS, the HORMA domain of Atg13 directly interacts with the N-terminal IDR of Atg9 (Suzuki et al., 2015). Furthermore, the Atg9-containing vesicles directly associate with Atg17 (Sekito et al., 2009). As a result, Atg9 is incorporated into the supra complex of Atg1-Atg13-Atg17-Atg31-Atg29 (Figure 2.5).

A

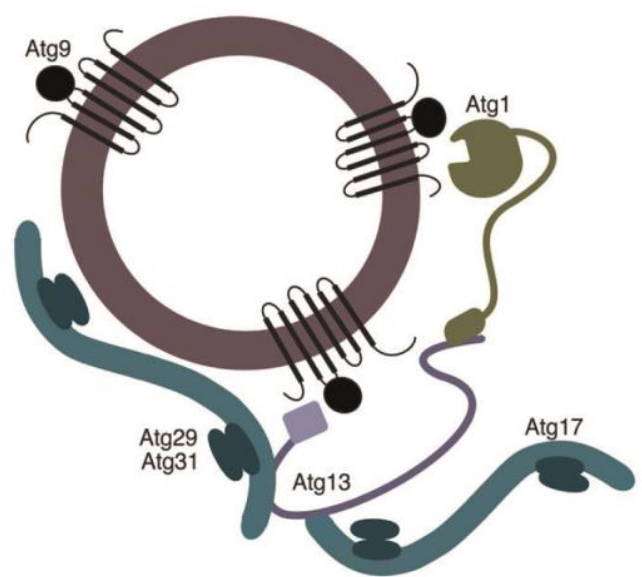

B

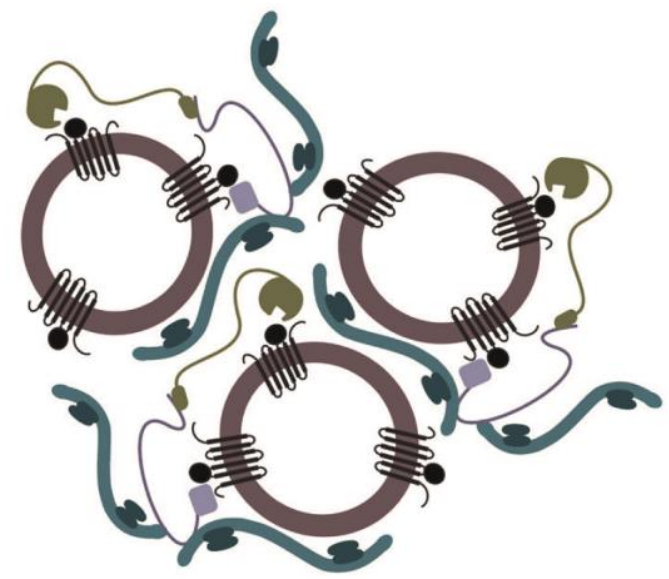

Figure 2.5: Incorporation of the Atg9-containing vesicles into the supra complex of Atg1-Atg13-Atg17-Atg31-Atg29 (modified from Hurley and Young (2017))

(A) Upon autophagy induction Atg9 (black) is phosphorylated by Atg1 (green) and its N-terminal domain directly interacts with the HORMA domain of Atg13 (violet). Atg13 makes the contact to the Atg17-Atg31-Atg29 (light and dark blue) subcomplex which leads to an association of Atg9 with Atg17. (B) Atg13 bridges the interaction between several Atg1 kinase complexes, thereby forming a mesh-like structure in which the Atg9-containing vesicles (brown) are incorporated.

The S-shaped architecture of the Atg17-homodimer is thought to tether the Atg9containing vesicles to each other resulting in their fusion and the formation of the initial membrane source of the phagophore (Ragusa et al., 2012; Rao et al., 2016). High resolution microscopy uncovered that approximately three Atg9-containing vesicles are assembled at the PAS. Furthermore, it was reported that no additional Atg9-containing vesicles are recruited to the phagophore in later steps of autophagosome formation (Yamamoto et al., 2012).

After recruitment to the PAS, Atg9 is phosphorylated by Atg1. Phosphorylation of Atg9 does not influence its localization at the PAS but is necessary for the recruitment of further components of the autophagic core machinery including the PI3-kinase complex and the Atg2-Atg18 complex (Shintani et al., 2001; Wang et al., 
2001; Papinski and Kraft, 2014; Suzuki et al., 2015). Before or after closure of the autophagosome, Atg9 is recycled to its peripheral pool. The retrograde transport requires the Atg1-Atg13 complex as well as the Atg2-Atg18 complex (Reggiori et al., 2004; Yamamoto et al., 2012).

\subsubsection{PI3-kinase complex}

The presence of phosphatidylinositol 3-phosphate (PI3P) at the autophagic membrane is an essential factor for autophagosome formation. PI3P is an important signaling molecule which concentrates PI3P-binding proteins at the phagophore. Apart from autophagy, PI3P is involved in endocytic transport and signaling as well as cytokinesis (Reidick et al., 2017).

In yeast, PI3P is generated by Vps34, which is the only class III PI3-kinase in this organism (Schu et al., 1993). It exclusively phosphorylates the 3-hydroxyl group of the inositol ring of phosphatidylinositol (PI) (Schu et al., 1993; Stack and Emr, 1994). To conduct various cellular functions, Vps34 acts in two distinct complexes (I and II) (Figure 2.6). Core components of these complexes are Vps34 itself and the putative protein kinase Vps15 (Stack et al., 1995). Vps15 mediates the membrane association of the PI3-kinase complexes and therefore their correct localization in the cell. Although Vps15 is myristoylated at its N-terminus, membrane binding of Vps15 is not only dependent on myristoylation (Herman et al., 1991). Another component present in both complexes is Vps30 (also called Atg6). The association of Vps30 with the PI3-kinase complexes is bridged by either of the two proteins Atg14 or Vps38 which are specific for the respective complexes (Kihara et al., 2001). PI3-kinase complex II is mainly found on endosomal membranes and is required for vacuolar protein sorting but has no function in autophagy (Kihara et al., 2001). It seems to have a crucial role in recruiting the retromer complex which is required for the retrograde transport from endosomes to Golgi (Burda et al., 2002). The unique component of complex II is Vps38 (Kihara et al., 2001).

In contrast, PI3-kinase complex I is essential for unselective as well as selective autophagic processes. Unique components of complex I are Atg14, which targets the complex to the PAS, and Atg38 (Obara et al., 2006; Araki et al., 2013). While membrane association of PI3-kinase complex I is mediated by Vps15, its PAS 
localization depends on Atg14. Proteins required for the PAS recruitment of Atg14 are the Atg1 kinase complex components Atg17 and Atg13 (via the HORMA domain) as well as Atg9 (Kuninori Suzuki et al., 2007; Jao et al., 2013).

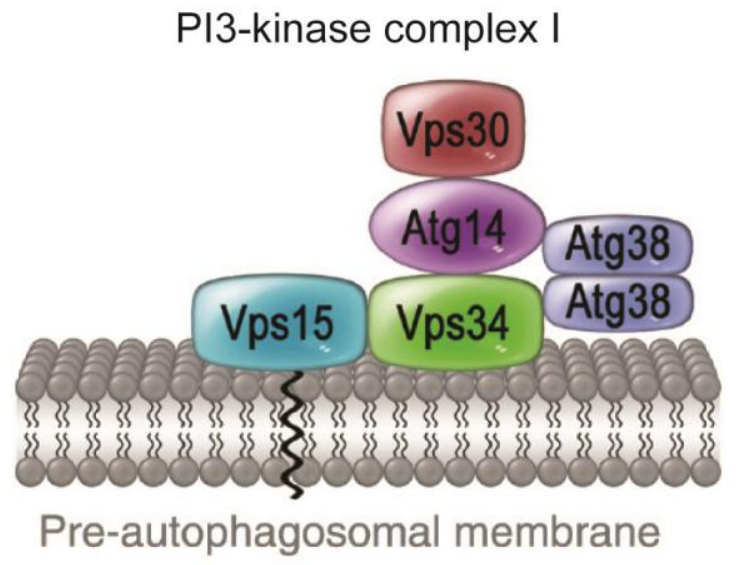

$$
1
$$

Autophagosome formation

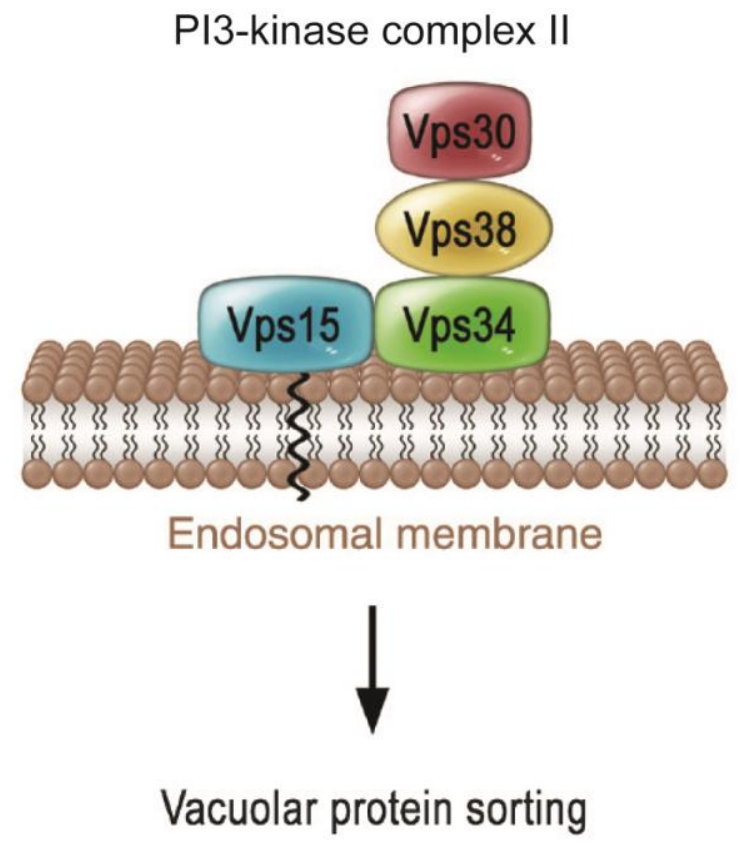

Figure 2.6: Composition of the PI3-kinase complexes I and II (modified from Reidick et al. (2017))

Both complexes share three common subunits which are the PI3-kinase Vps34, the myristoylated putative kinase Vps15 and Vps30. The association of Vps30 with the PI3-kinase core complex of Vps34 and Vps15 is bridged by either of the two proteins Atg14 or Vps38 which are specific for the distinct complexes. Complex I is essential for autophagic processes and comprises the unique components Atg14 and Atg38. Atg14 targets complex I to the PAS. Atg38 forms a homodimer and interacts with Vps34 and Atg14. Complex II localizes to the endosomal membrane and is required for vacuolar protein sorting. Its unique component is Vps38.

The C-terminal part of Atg38 mediates the formation of a homodimer while the N-terminal part interacts with Vps34 and Atg14. Atg38 seems to strengthen the interaction of the Vps15-Vps34 and the Atg14-Vps30 subcomplexes (Araki et al., 2013). However, using recombinant proteins, it was shown that the components of complex I can be co-purified in the absence of Atg38 (Ohashi et al., 2016). Therefore, its function in the complex remains elusive. Nevertheless, deletion of ATG38 significantly reduced the autophagic process (Araki et al., 2013).

\subsubsection{Two ubiquitin-like conjugation systems}

Two ubiquitin-like conjugation systems belong to the core autophagic machinery. They act downstream of the already described autophagic components and are required for the elongation of the autophagic membrane. Both conjugation systems 
cooperate to result into the transfer of the ubiquitin-like protein Atg8 to the lipid phosphatidylethanolamine (PE). The second ubiquitin-like protein is Atg12 which is transferred to Atg5 during the conjugation cascade. The formation of the Atg12 Atg5 conjugate is a prerequisite for the Atg8 lipidation (Mizushima et al., 1998; Ichimura et al., 2000; Hanada et al., 2007; Wen and Klionsky, 2016).

Atg8 is initially synthesized with an additional C-terminal arginine. This is directly removed by the cysteine protease Atg4 to expose a C-terminal glycine, thereby preparing Atg8 for the conjugation reaction (Figure 2.7 A; Kirisako et al., 2000). In contrast to this, Atg12 does not require any processing prior to the conjugation reaction. To start the conjugation cascade both, Atg8 and Atg12, are activated by the E1-like enzyme Atg7. In an ATP-dependent reaction the C-terminal glycines of the ubiquitin-like proteins form a thioester bond with the catalytic cysteine of Atg7 (Figure 2.7 B). Atg7 acts as a homodimer, of which one subunit binds the ubiquitinlike proteins and the other one recruits the respective E2-like enzymes Atg3 (for Atg8) or Atg10 (for Atg12). In these Atg3-Atg7 Atg8 or Atg10-Atg7 Atg12 complexes, the activated Atg8 and Atg12 are transferred to the catalytic cysteines of their respective E2-like enzymes forming the thioester intermediates Atg8 Atg3 and Atg12 Atg10 (Figure 2.7 C; Mizushima et al., 1998; Ichimura et al., 2000; Komatsu et al., 2001; Noda et al., 2011; Hong et al., 2011).

Finally, Atg10 is able to directly bind to Atg5 and facilitates the E3-independent conjugation of the C-terminal glycine of Atg12 with lysine 149 of Atg5 (Figure 2.7 D; Shintani et al., 1999; Yamaguchi et al., 2012). The Atg12 Atg5 conjugate is constitutively formed and there is no deconjugation reaction known. Atg5 is another ubiquitin-like protein comprising two ubiquitin-like domains that are connected by a helix rich region. The C-terminal ubiquitin-like domain is responsible for the interaction with Atg10. In addition to its covalent interaction with Atg12, Atg5 noncovalently binds Atg16. Atg16 forms a dimer consisting of two N-terminal Atg5binding domains as well as a C-terminal dimeric coiled-coil domain (CCD). The dimer of Atg16 promotes homodimerization of the whole Atg12 Atg5/Atg16 complex (Figure 2.7 E; Mizushima et al., 1999; Kuma et al., 2002; Fujioka et al., 2010; Sakoh-Nakatogawa et al., 2013). 


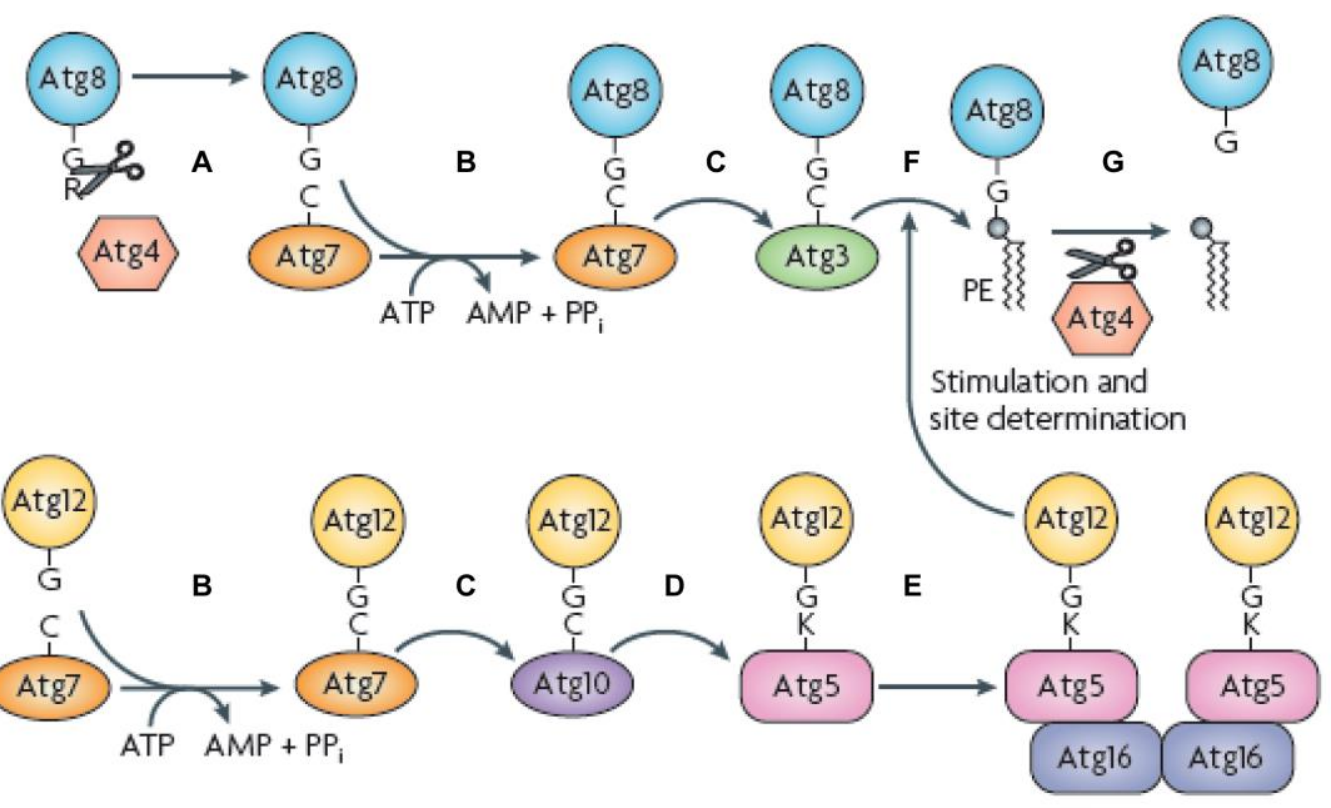

Figure 2.7: The two ubiquitin-like conjugation systems in autophagy (modified from Nakatogawa et al. (2009))

(A) The C-terminal arginine of Atg8 is removed by the cysteine protease Atg4. Thereby, a glycine residue is exposed which is required for the following conjugation cascade. (B) The ubiquitin-like proteins Atg8 and Atg12 are activated by the E1-like enzyme Atg7. In an ATP-consuming reaction their C-terminal glycines form a thioester bond with the catalytical cysteine of Atg7. (C) Facilitated by Atg7, Atg8 and Atg12 are then transferred to the E2-like enzymes Atg3 and Atg10 respectively. (D) Via its C-terminal glycine, Atg12 is finally linked to a lysine of Atg5. (E) Atg5 noncovalently interacts with Atg16 which promotes the formation of an homodimeric complex. (F) The Atg12 Atg5/Atg16 complex acts as the E3-like enzyme for the transfer of Atg8 from Atg3 to PE. (G) Atg4 is able to hydrolyze the bond between Atg8 and PE, a function that is required in later stages of autophagy.

The Atg12 Atg5/Atg16 complex acts as the E3-like enzyme for the conjugation of Atg8 to PE. Atg12 interacts with Atg3, recognizes as well as activates its catalytic center and thereby facilitates the transfer of the C-terminal glycine of Atg8 to the amine moiety of PE (Figure 2.7 F; Ichimura et al., 2000; Hanada et al., 2007; SakohNakatogawa et al., 2013). The lipidation of Atg8 has been reconstituted in vitro with recombinantly expressed and purified components. Notably, Atg16 is not required for the conjugation of Atg8 to PE in vitro and does not display an E3-like function, most likely due to the artificial conditions used. However, in vivo it is essential to target the Atg12 Atg5 conjugate to the autophagic membrane and thereby positions the Atg8 Atg3 conjugate in close proximity to PE (Suzuki et al., 2001; Ichimura et al., 2004; Hanada et al., 2007; Sakoh-Nakatogawa et al., 2013).

Besides its function in cleaving the C-terminal arginine of Atg8, Atg4 is also able to hydrolyze the bond between Atg8 and PE (Figure $2.7 \mathrm{G}$ ). This function of Atg4 is 
required during autophagosome maturation to release Atg8 from the outer autophagic membrane (chap. 2.4). Furthermore, it is proposed to remove randomly generated Atg8-PE from non-autophagic membranes (Kirisako et al., 2000; Nakatogawa et al., 2012a; Nair et al., 2012).

\subsubsection{Detailed view on the function of Atg8}

The small protein Atg8 (13.6 kDa) is a member of a highly conserved family of ubiquitin-like proteins that is essential for autophagosome formation. While Atg8 is the single member of this family in yeast, mammalian cells harbor several homologues that can be subdivided into three groups: microtubule-associated protein 1 light-chain 3 (MAP1LC3 hereafter referred to as LC3) proteins, $\gamma$ aminobutyric acid type A receptor binding proteins (GABARAP) and Golgiassociated ATPase enhancer of $16 \mathrm{kDa}$ (GATE-16) proteins (Slobodkin and Elazar, 2013). The members of the LC3 group are proposed to mediate early stages of autophagosome biogenesis while the GABARAP group seems to be involved in later steps (Weidberg et al., 2010). LC3B a member of the LC3-group is the best characterized member of the Atg8-family in higher eukaryotes and is regularly used to monitor autophagy progression in mammalian cells (Slobodkin and Elazar, 2013). Likewise, Atg8 is often used as a marker protein for autophagic membranes due to its covalent association with PE. Furthermore, it is used to measure the autophagic flux in yeast (Torggler et al., 2017).

Atg8 is essential for phagophore expansion and it was reported that there is a direct correlation between the amount of Atg8-PE on the autophagic membrane and the size of the autophagosome. Thereby, the number of forming autophagosomes is not affected (Xie et al., 2008). Considering this effect of Atg8, it is not surprising that the ATG8 expression is highly induced upon starvation (about tenfold) (Xie et al., 2008). Initially Atg8-PE is present on both sides of the growing phagophore. On the inner membrane, the concave side of the phagophore, Atg8-PE is responsible for the recognition of the cargo in selective types of autophagy (Figure 2.9 B). Cargo recognition is mediated by special cargo receptors which interact with Atg8 and thereby link the cargo to the autophagic machinery (Stolz et al., 2014; Farré and Subramani, 2016). Typical cargo receptors in yeast are for example the Cvt-complex 
receptor Atg19, the mitophagy receptor Atg32 or the pexophagy receptor Atg36 (or Atg30 in Pichia pastoris) (Shintani et al., 2002; Farré et al., 2008; Kanki et al., 2009b; Okamoto et al., 2009; Motley et al., 2012). These receptors were found to bind to Atg8 via one or more common Atg8-interacting motifs (AIM). AIMs are also called WXXL-motifs or LC3-interacting regions (LIR) for mammalian interactors of Atg8family members (Noda et al., 2008; Farré and Subramani, 2016).

One of the first identified AIMs in yeast proteins was that of Atg19. Its interaction with Atg8 is mediated by a WEEL sequence at the very C-terminus of Atg19 (Shintani et al., 2002; Noda et al., 2008). A sequence alignment similar to that shown in Figure $2.8 \mathrm{~B}$ of 26 functional AIMs from different proteins and organisms resulted in the following consensus sequence of canonical AIMs: $\mathrm{X}_{-3} \mathrm{X}_{-2} \mathrm{X}-1[\mathrm{~W} / \mathrm{F} / \mathrm{L}]_{0} \mathrm{X}_{+1} \mathrm{X}_{+2}[\mathrm{~L} / \mathrm{I} / \mathrm{V}]+3$, where X represents any amino acid (Figure 2.8 C; Alemu et al., 2012). The consensus sequence reveals that an aromatic amino acid at position 0 and a hydrophobic amino acid at position +3 are fundamental characteristics of canonical AIMs. Furthermore, it was reported that the positions $-3,-2$ and -1 as well as +1 and +2 are preferably occupied by acidic amino acids. The acidic amino acids may exert ionic interactions with corresponding arginine or lysine residues of the Atg8-family protein (Noda et al., 2010; Alemu et al., 2012). The acidic environment of the AIM is reported to be also supported by phosphorylation of serine and threonine residues in the AIM or nearby. This was for example observed for the mitophagy receptor Atg32 or the pexophagy receptor Atg36 (Farré et al., 2013).

A

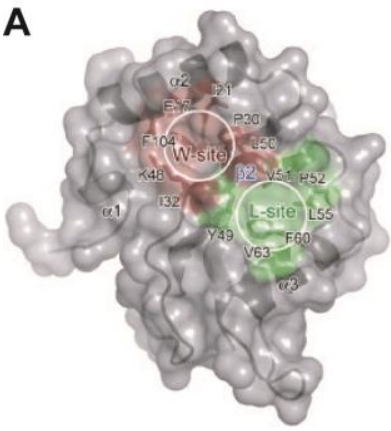

B

$\begin{array}{lll}\text { Btg19 } & \text { AIM sequence } & \text { Binding partner } \\ \text { ALTEEL } & \text { Atg8 } \\ \text { Atg32 } & \text { SGSWQAIQP } & \text { Atg8 } \\ \text { Atg3 } & \text { VGDWEDLQD } & \text { Atg8 } \\ \text { Atg4B } & \text { TLTYDTLRF } & \text { LC3 } \\ \text { p62 } & \text { DDDWTHLSS } & \text { LC3 } \\ \text { NBR1 } & \text { SEDYIIILP } & \text { LC3 } \\ \text { CRT } & \text { EDDWDFLPP } & \text { GABARAP } \\ \text { CHC } & \text { TPDWIFLLR } & \text { GABARAP } \\ \text { Nix } & \text { NSSWVELPM } & \text { GABARAP } \\ \text { (PSSM) } & \text { DDDWVFVPM } & \text { GABARAP } \\ \text { SEPA-1 } & \text { (TYGYQELDD) } & \text { LGG-1 } \\ & \text { W }_{0} \text { L }_{3} & \end{array}$

C

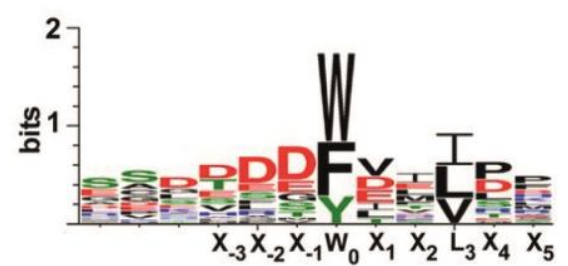

Figure 2.8: Structural and sequential requirements for AIM-dependent Atg8 interaction

(A) Displayed is the structure of S. cerevisiae Atg8 (PDB code: 2zpn). The two hydrophobic pockets, called the $\mathrm{W}$ - and L-site, that are responsible for the recognition of the aromatic residue and the hydrophobic residue in AIMs are colored red and green respectively (modified from Noda et al. (2010)). (B) Sequence alignment of functional AIMs derived from different Atg8-family interacting proteins from different organisms (modified from Noda et al. (2010)). (C) Consensus sequence derived from the sequence alignment of 26 reported AIMs. The height of the letters corresponds to the relative frequency of this amino acid at the respective position while the height of the total stack 
indicates the sequence conservation. Color code: black=hydrophobic; red=acidic; blue=basic; green=hydroxyl group (modified from Alemu et al. (2012)).

However, AIMs are not only present in cargo receptors but also in core autophagic proteins like Atg1 as well as in Atg8-modifing enzymes like Atg3 and Atg4 (Yamaguchi et al., 2010; Kraft et al., 2012; Nakatogawa, et al., 2012b; Abreu et al., 2017). Furthermore, members of the GABARAP family were reported to interact with non-autophagic proteins in an AIM-like manner. Therefore, the AIM seems to be a general feature of Atg8-family interactors independent of autophagy (Mohrlüder et al., 2007; Thielmann et al., 2009; Noda et al., 2010).

Apart from the canonical AIMs, also cryptic AIMs exist which are not composed of a conserved sequence motif but rather structurally adopt the properties of canonical AIMs. Cryptic AIMs were for example identified in Atg19 and also in Atg12 (Noda et al., 2008; Kaufmann et al., 2014; Sawa-Makarska et al., 2014). The cryptic AIM of Atg12 is involved in the proposed function of Atg8-PE on the convex side of the phagophore. Here, Atg8-PE forms a coat-like structure with the Atg12 Atg5/Atg16 complex (Figure 2.9). In vitro studies using giant unilamellar vesicles (GUVs) showed that Atg8-PE recruits the Atg12 Atg5 conjugate to the membrane via the interaction with a cryptic AIM of Atg12. Furthermore, the resulting Atg8-PEAtg12 Atg5 oligomers were arranged by Atg16 into a stable membrane-scaffold with a mesh-like structure. This scaffold on the outer membrane of the phagophore is thought to stabilize the forming phagophore and supports the formation of productive autophagosomes (Kaufmann et al., 2014).

All Atg8-family members share the same structural elements. They are composed of a ubiquitin-core fold (C-terminal domain) and a N-terminal helical domain. The two $\mathrm{N}$-terminal tandem $\alpha$-helices $\left(\alpha_{1}\right.$ and $\alpha_{2}$ ) are a conserved structural and functional element unique to Atg8-family members and makes them distinguishable from other ubiquitin-like proteins (e.g. Atg12 or Atg5) (Noda et al., 2008; Noda et al., 2010). The N-terminal $\alpha$-helices of Atg8 are required for efficient autophagy and were reported to have an arm-like structure which can comprise an open or closed conformation. The different conformations of the N-terminal domain may determine different functions of Atg8 (Schwarten et al., 2010; Kumeta et al., 2010; Wu et al., 2015). 

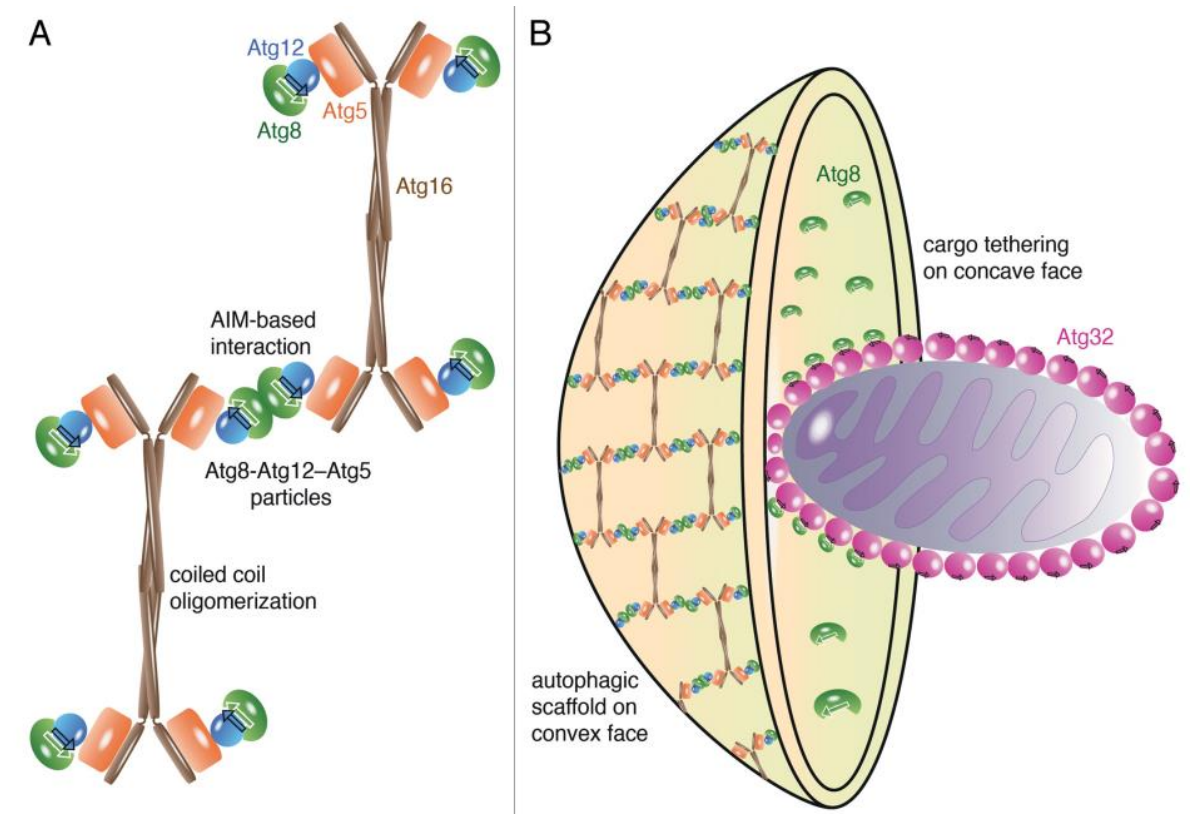

Figure 2.9: Proposed structure of the coat-like scaffolding complex of Atg8-Atg12-Atg5-Atg16 on the surface of the phagophore (Kaufmann and Wollert, 2014)

(A) Atg8 (green) directly binds Atg12 (blue) through a cryptic AIM in Atg12 and as a consequence recruits the Atg12 Atg5/Atg16 complex to the membrane. Atg16 (brown) organizes the resulting oligomers into a mesh-like scaffold. (B) The resulting coat-like structure is located on the convex side of the phagophore while on the concave side Atg8-PE recruits cargos (represented by a mitochondrion) through the interaction with special cargo receptors (represented by Atg32 in pink).

On the surface of the ubiquitin core, two hydrophobic pockets are formed (Figure $2.8 \mathrm{~A}$ in red and green). Together, the two hydrophobic pockets mediate the interaction with AIMs of Atg8-family interactors. These pockets are named W- and L-sites since they interact with the aromatic residue on position 0 and the hydrophobic residue on position 3 of the AIM respectively. Furthermore, an exposed $\beta$-strand $\left(\beta_{2}\right)$ is located between the $\mathrm{W}$ - and L-site which participates in AIM-binding by the formation of an intermolecular $\beta$-sheet (Noda et al., 2008; Noda et al., 2010). The two N-terminal $\alpha$-helices of Atg8 seem to further promote oligomerization of Atg8 which probably coincides with another function of Atg8. In vitro studies showed that Atg8 might be able to tether and hemifuse liposomes while mammalian members are even able to fully fuse liposomes (Nakatogawa et al., 2007; Weidberg et al., 2011a).

\subsection{Membrane sources of autophagosomes}

The phagophore seems to be formed de novo which means that it is not directly generated from a single pre-existing organelle. Phagophore formation is nucleated 
at the PAS presumably by the fusion of several Atg9-containing vesicles which act as the initial membrane source (Ragusa et al., 2012; Rao et al., 2016). It was estimated that Atg9-containing vesicles only supply about $2 \%$ of the lipids of the growing phagophore (Yamamoto et al., 2012). After nucleation, the elongation of the autophagic membrane therefore requires the addition of further lipids. One model for the delivery of lipids to the elongating phagophore is their addition by vesicles from various membrane sources. A variety of studies have implicated the ER, the Golgi apparatus, endosomes, mitochondria and the plasma membrane as potential membrane donors (Hamasaki et al., 2005; Hailey et al., 2010; Geng et al., 2010; Ravikumar et al., 2010; Puri et al., 2013).

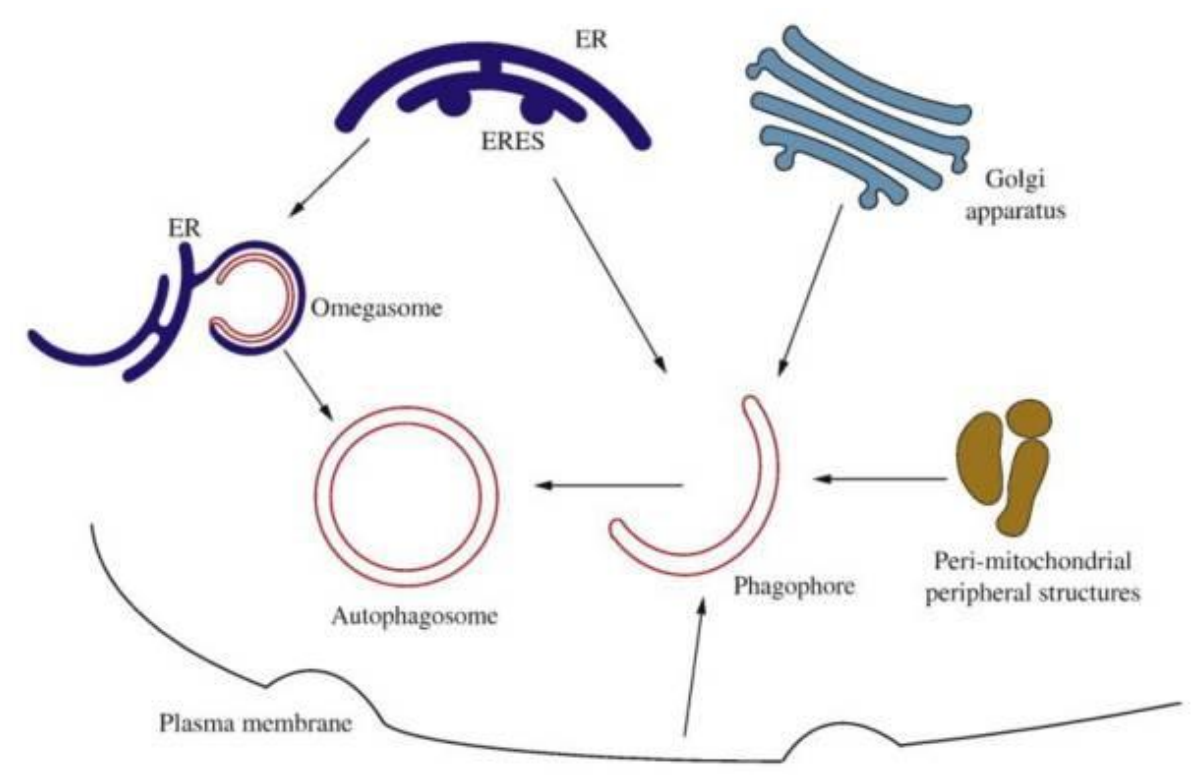

Figure 2.10: Proposed membrane sources for autophagosome formation (Wen and Klionsky, 2016)

Potential membrane sources for the growing phagophore are the ER, namely the ER exit sites (ERES), the Golgi apparatus, mitochondria or the plasma membrane. Lipids are thought to be delivered by the addition of vesicles. In mammalian cells lipids are also supplied by the lateral movement from a specialized membrane domain of the ER, called the omegasome.

A second model for the supply of lipids to the phagophore has been proposed for mammalian cells. It involves the lateral movement of lipids from an ER-derived unique structure, termed the omegasome, into the phagophore (Figure 2.10). The omegasome is a PI3P enriched domain of the ER that is thought to serve as a platform for autophagosome formation (Roberts and Ktistakis, 2013). Interestingly, a recent study reported a similar single-membrane structure in yeast that emerges from the outer nuclear membrane and seems to specifically sequester the Cvtcomplex. It is termed the alphasome (Baba et al., 2018). 
One of the first debated membrane sources for the autophagosome formation was the ER (Dunn, 1990). It was reported that autophagy is blocked in strains that are defective in the early stages of the secretory pathway, including the ER-to-Golgi transport (Ishihara et al., 2001). The transport of secretory cargos from the ER to the Golgi is mediated by COPII vesicles which are assembled at specialized membrane domains of the ER, called the ER exit sites (ERES) (Tang et al., 2005). Autophagy defects were reported for mutants of the cargo-binding subunits Sec23 and Sec 24 of the COPII coat as well as the ERES specific proteins Sec12 and Sec16 suggesting a direct contribution of ERES and COPII vesicles to autophagosome formation (Ishihara et al., 2001; Graef et al., 2013). This is supported by recent findings that the edges of the growing phagophore are not only adjacent to the ERES but form a functional Atg2-dependent contact site with the ER (Suzuki et al., 2013; Graef et al., 2013; Gomez-Sanchez et al., 2018; Kotani et al., 2018).

The direct participation of COPII vesicles in phagophore formation is further supported by the observation that they accumulate at the PAS when autophagy is blocked (Tan et al., 2013). Furthermore, a physical interaction of COPII coat components with elements of the autophagic core machinery including the Atg1 kinase and PI3-kinase complexes was reported (Graef et al., 2013). Two proteins have been discussed to tether COPII vesicles to the PAS. One of these proteins is Atg1 which was shown to bind highly curved vesicles (Ragusa et al., 2012). The other suggested tether is the multimeric transport protein particle (TRAPP) III complex which is a guanine nucleotide exchange factor (GEF) of the Rab guanosine triphosphatase (GTPase) Ypt1. Ypt1 and the TRAPP III complex are both involved in autophagy (Meiling-Wesse et al., 2005; Lynch-Day et al., 2010). Ypt1 was shown to interact with Atg1, thereby facilitating its recruitment to the PAS (Wang et al., 2013). The TRAPP III complex is able to bind COPII vesicles and is recruited to the PAS via its Trs85 subdomain by the interaction with Atg17. Trs85 was further reported to directly interact with Atg9 (Lynch-Day et al., 2010; Shirahama-Noda et al., 2013; Tan et al., 2013). Due to their ability to bind both types of vesicles, the two suggested COPII tethers have been discussed to facilitate a heterotypic fusion of Atg9containing vesicles with COPII vesicles (Ge et al., 2014; Davis and Ferro-Novick, 2015). 


\subsection{Autophagosome maturation and fusion with the vacuole}

The final step of phagophore expansion is the closure to form the doublemembraned autophagosome which completely encloses the cargo. The completion of autophagosomes is likely to include the scission and fusion of membranes at the edges of the growing phagophore to separate the inner and outer membrane. Almost nothing is known about these closing mechanisms (Carlsson and Simonsen, 2015; Reggiori and Ungermann, 2017). Atg9 and the interacting Atg2-Atg18 complex were reported to localize to the edges of the phagophore and could therefore be putative factors that mediate its closure (Suzuki et al., 2013; Graef et al., 2013). Furthermore, some mammalian members of the Atg8-family were shown to have a membrane tethering and fusing function which is mediated by their N-terminal $\alpha$-helix. Some of these members are further suggested to act in later steps of autophagosome formation and are therefore discussed as potential closure factors (Weidberg et al., 2010; Weidberg et al., 2011b).

The closure of the autophagosome and its subsequent fusion with the vacuole must be tightly regulated processes since a premature fusion of the growing phagophore with the vacuole would not result in the delivery of the cargo (Wen and Klionsky, 2016). The presence of Atg proteins on the phagophore could be a likely inhibitor for the recruitment or activation of the fusion machinery. After closure and before fusion with the vacuole, the autophagosomes need to be matured which is defined by the removal of Atg proteins from the autophagosomal surface. During this process, the Atg proteins are recycled for reuse and the fusion machinery may be recruited or activated. Two important steps of autophagosome maturation are the removal of PI3P and Atg8 from the autophagosomal surface which probably facilitate the dissociation of further Atg proteins (Figure $2.11 \mathrm{~A}$; Reggiori and Ungermann, 2017).

As described above, the protease Atg4 does not only remove the C-terminal arginine of Atg8 to promote its conjugation to PE but is also able to hydrolyze the bond between Atg8 and PE and thereby recycles Atg8 from its lipid anchor (Kirisako et al., 2000). Atg4 mainly shows a dispersed cytosolic localization and only transiently associates with the PAS (Nakatogawa, et al., 2012a; Abreu et al., 2017). It was 
suggested that cytosolic Atg4 constitutively processes the C-terminus of Atg8 while the PAS associated Atg4 hydrolyzes Atg8-PE (Reggiori and Ungermann, 2017).

Recently it was shown that the phosphorylation of Atg4 by Atg1 inhibits its proteolytic activity, an event that appears to exclusively occur at the PAS (SanchezWandelmer et al., 2017). Phosphorylated Atg4 is unable to interact with Atg8 which presumably prevents the cleavage of Atg8-PE at premature autophagosomes (Sanchez-Wandelmer et al., 2017). This is further supported by the observation that the association of Atg4 with the PAS is altered in ATG1 deleted cells (Abreu et al., 2017). Probably, the phosphorylation inhibits the binding properties of one or both of the identified AIMs of Atg4 that seem to cooperatively mediate the interaction with Atg8 (Abreu et al., 2017; Sanchez-Wandelmer et al., 2017). One of these AIMs was shown to specifically recognize Atg8-PE while the other appears to regulate the constitutive interaction with Atg8. The mutation of both Atg4 AIMs impaired the cleavage of Atg8 from PE. Upon closure of the autophagosome, the dissociation or inactivation of Atg1 would enable Atg4 to hydrolyze Atg8-PE (Abreu et al., 2017).

A second important step during autophagosome maturation is the hydrolysis of PI3P into PI. This step is mediated by the PI3P-specific phosphatase Ymr1. Yeast cells deleted for YMR1 accumulate autophagosomes in the cytosol which still harbor Atg proteins on their surface and are covered with PI3P. This was even more pronounced in cells that were additionally deleted for one of the general phosphoinositide phosphatases Sj12 and Sjl3. Furthermore, Ymr1 and Sjl3 associate with the PAS upon autophagy induction. So far, it is not known how the activity of both proteins is regulated during the formation and maturation of the autophagosome (Parrish et al., 2004; Cebollero et al., 2012; Cheng et al., 2014).

The fusion of matured autophagosomes with the vacuole is related to other fusion events of intracellular vesicle trafficking, like the endosome-vacuole fusion. This includes the action of a specific Rab GTPase in combination with its respective GEF, a tethering factor as well as soluble $\mathrm{N}$-ethylmaleimide-sensitive-factor attachment receptor (SNARE) proteins (Figure 2.11 B; Reggiori and Ungermann, 2017). Rab GTPases are known to regulate intracellular membrane traffic. They can act as molecular switches by cycling between their soluble GDP-bound (inactive) and the membrane associated GTP-bound (active) form. Membrane association is mediated 
by one or two geranylgeranyl groups at the C-terminus of the Rab GTPase. The replacement of GDP with GTP is mediated by GEFs and leads to membrane association as well as activation of the Rab GTPase which is then able to recruit effector proteins (Goody et al., 2017).
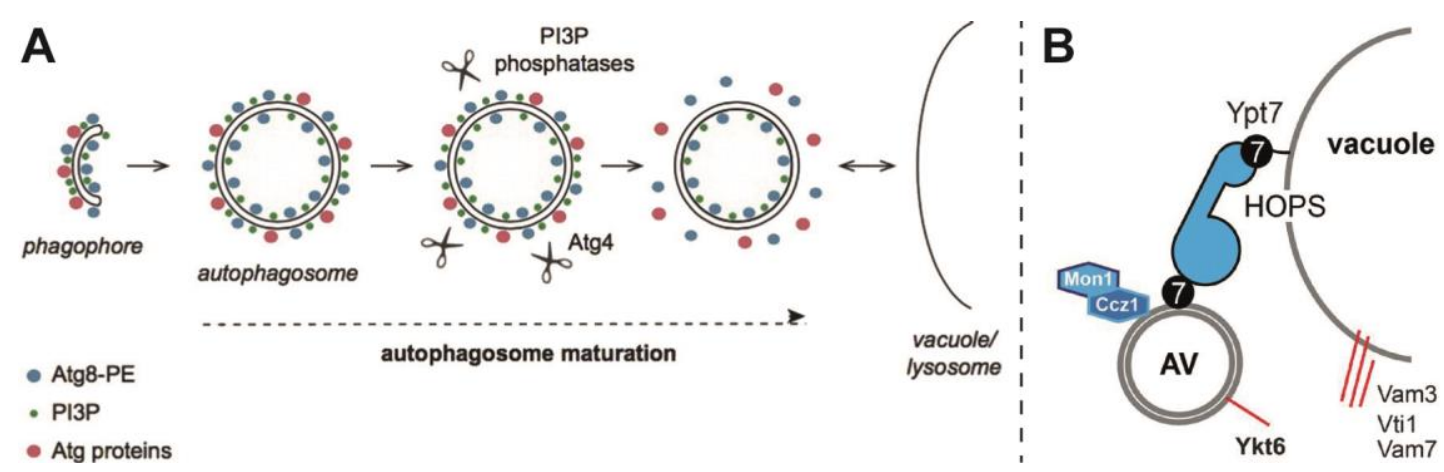

Figure 2.11: Model for autophagosome maturation and fusion with the vacuole (modified from Reggiori and Ungermann (2017) and Gao et al. (2018b))

(A) After closure and before fusion with the vacuole, autophagosomes need to be matured. This step includes the hydrolysis of PI3P by the specific PI3P phosphatase Ymr1 as well as the deconjugation of Atg8-PE by the protease Atg4. It results in the dissociation of Atg proteins from the autophagosomal surface. (B) The Rab GTPase Ypt7 is targeted to the vacuole and the autophagosome (AV) as well as activated by its GEF complex Mon1-Ccz1. Activated Ypt7 binds to the HOPS complex. As a consequence, the autophagosome is tethered to the vacuolar membrane. The HOPS complex further promotes the assembly of a SNARE complex to facilitate the fusion of autophagosome and vacuole. The Q-SNAREs Vam3, Vti1 and Vam7 act on the vacuolar membrane while the R-SNARE Ykt6 acts on the autophagosomal membrane.

Autophagosomes use the same machinery for vacuolar fusion that is also required for the fusion of endosomes with the vacuole. This includes the RAB7-homolog Ypt7 as the specific Rab GTPase which requires the Mon1-Ccz1 complex as a GEF for activation (Kirisako et al., 1999; Wang et al., 2002; Nordmann et al., 2010). The Mon1-Ccz1 complex localizes to endosomes, vacuoles and autophagosomes (Wang et al., 2002; Wang et al., 2003; Gao et al., 2018a). Recently, it was found to be targeted to the autophagosomal surface via the interaction with Atg8. Atg8 interaction is mediated by a C-terminal AIM of Ccz1 which does not affect the GEF activity of the complex. Thereby, the Mon1-Ccz1 complex can selectively recruit Ypt7 to autophagosomes and activate it (Gao et al., 2018a). Autophagosomal association of Ypt7 further requires the presence of PI3P (Bas et al., 2018).

Activated Ypt7 further interacts with the homotypic vacuole fusion and protein sorting (HOPS) complex which is bound to the vacuolar membrane. The HOPS complex is a hexameric tethering complex which is able to tether Ypt7 positive membranes. Furthermore, it triggers the assembly of SNARE proteins to facilitate 
the fusion of the tethered membranes (Hickey and Wickner, 2010; Krämer and Ungermann, 2011; Ho and Stroupe, 2015).

SNAREs are membrane-anchored proteins that comprise heptad repeat domains (SNARE domains) with a central arginine (R-SNARE) or glutamine (Q-SNARE) residue. SNAREs interact with each other to form SNARE complexes consisting of three Q-SNAREs and one R-SNARE. They can be located on opposing membranes (trans-SNARE complex) and promote membrane fusion by zippering their SNARE domains (Wickner and Rizo, 2017). The Q-SNAREs Vam3, Vam7 and Vti1 as well as the R-SNARE Ykt6 are essential for the vacuolar fusion of autophagosomes. Thereby, Vam3, Vam7 and Vti1 act on the vacuolar membrane while Ykt6 was recently identified to act on the autophagosomal membrane (Darsow et al., 1997; Fischer von Mollard and Stevens, 1999; Dilcher et al., 2001; Ishihara et al., 2001; Fader et al., 2009; Bas et al., 2018; Gao et al., 2018b).

Besides the tethering of vacuolar and autophagosomal membrane by the HOPS complex, Vam7 seems to interact with the Atg17-Atg31-Atg29 subcomplex of the Atg1 kinase complex. Impaired interaction of Vam7 and Atg17 reduces the vacuolar fusion of autophagosomes suggesting that there is a functional crosstalk between Atg17 and the fusion machinery (Liu and Klionsky, 2016).

\subsection{The role of PROPPINs in autophagy}

$\beta$-propellers that bind polyphosphoinositides (PROPPIN) are a family of eukaryotic $\mathrm{PI} 3 \mathrm{P}$ as well as phosphatidylinositol-3,5-bisphosphate $\left(\mathrm{PI} 3,5 \mathrm{P}_{2}\right)$ binding proteins which are involved in autophagy and are conserved from yeast to human. Yeasts harbor three homologous PROPPINs, Atg18, Atg21 and Hsv2, whereas mammals have four orthologues which are called WD40 repeat-containing proteins that interact with PIs (WIPI) (Barth et al., 2001; Guan et al., 2001; Barth et al., 2002; Stromhaug et al., 2004; Proikas-Cezanne et al., 2004; Krick et al., 2006). WIPI1 and WIPI2 are characterized as orthologues of Atg18 while WIPI3 and WIPI4 are more reminiscent of Hsv2. Atg21 has no orthologues in mammals and therefore appears to be restricted to yeasts (Polson et al., 2010). The following chapters detail the current knowledge of the structure and molecular function of the yeast PROPPINs. 


\subsubsection{Structure and membrane binding of PROPPINs}

PROPPINs belong to the family of WD40-repeat proteins which serve as platforms for multiple protein-protein interactions. WD40-repeat domains belong to the most abundant protein domains in eukaryotic proteomes and reside among the top ten interacting domains. Interestingly, so far no WD40-repeat domain is known that harbors an enzymatic activity. WD40 domains were named according to a conserved dipeptide of a tryptophan (W) and an aspartic acid (D) at the C-terminus of a 44-60 amino acid long sequence unit. Typically, these sequence units also contain a conserved GH dipeptide at position 11-24 from the N-terminus. Most of the WD40-repeat proteins comprise seven WD40-repeats each folding into a fourstranded antiparallel $\beta$-sheet (blade). The conserved GH and WD residues stabilize the WD40 fold by exhibiting hydrophobic interactions. Together the resulting seven blades are organized into a $\beta$-propeller architecture (Stirnimann et al., 2010; Xu and Min, 2011).

Structural information about the PROPPIN subfamily of WD40-repeat proteins has been obtained from the crystal structures of Kluyveromyces lactis Hsv2 (KlHsv2), Kluyveromyces marxianus Hsv2 (KmHsv2) and Pichia angusta Atg18 (PaAtg18) (Krick et al., 2012; Baskaran et al., 2012; Watanabe et al., 2012; Scacioc et al., 2017). As predicted, they fold into a seven-bladed $\beta$-propeller typical for WD40-repeat proteins where each blade comprises a four-stranded antiparallel $\beta$-sheet. The blades are numbered from one (N-terminus) to seven (C-terminus) while the single $\beta$-strands are termed A to D from the inner to the outer strand (Figure 2.12 A). The $\beta$-strands are linked by short loop regions which are named according to the blade they are part of and the $\beta$-strands they are connecting. For example, loop $2 \mathrm{AB}$ connects $\beta$-strands $A$ and $B$ of blade two. All loops connecting $\beta$-strands $B$ and $C$ as well as those linking one blade to the other are oriented towards the top side of the $\beta$-propeller while all $\mathrm{AB}$ and $\mathrm{CD}$ loops are exposed on the bottom side (Figure 2.12 B). Furthermore, the PROPPINs exhibit a non-velcro closure of the propeller. Here, the seventh blade is only formed by the C-terminal WD40-repeat and does not share any $\beta$-strands with the first blade like it is typical for most of the WD40-repeat proteins (Xu and Min, 2011; Krick et al., 2012; Baskaran et al., 2012; Watanabe et al., 2012; Scacioc et al., 2017). 
A

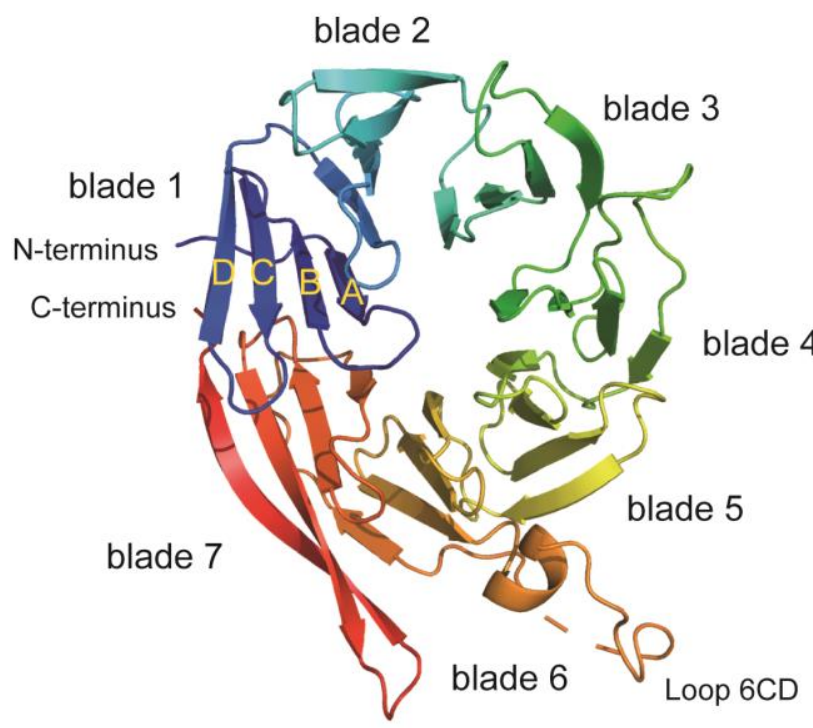

C

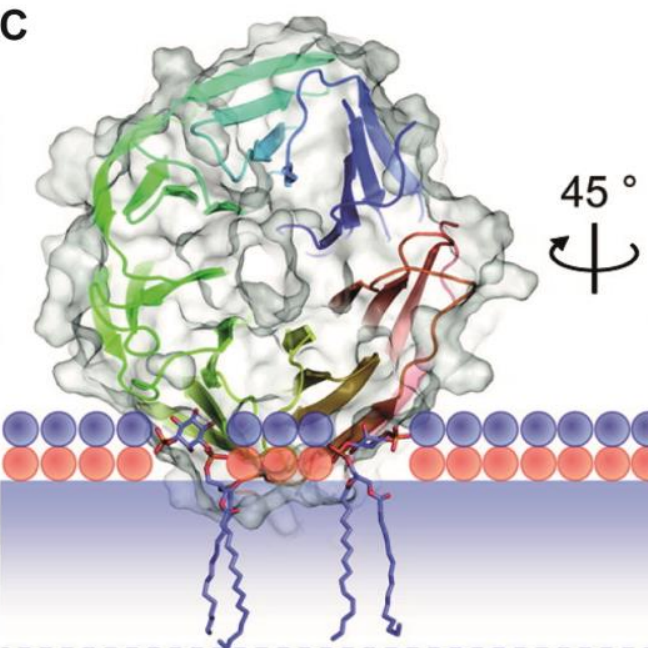

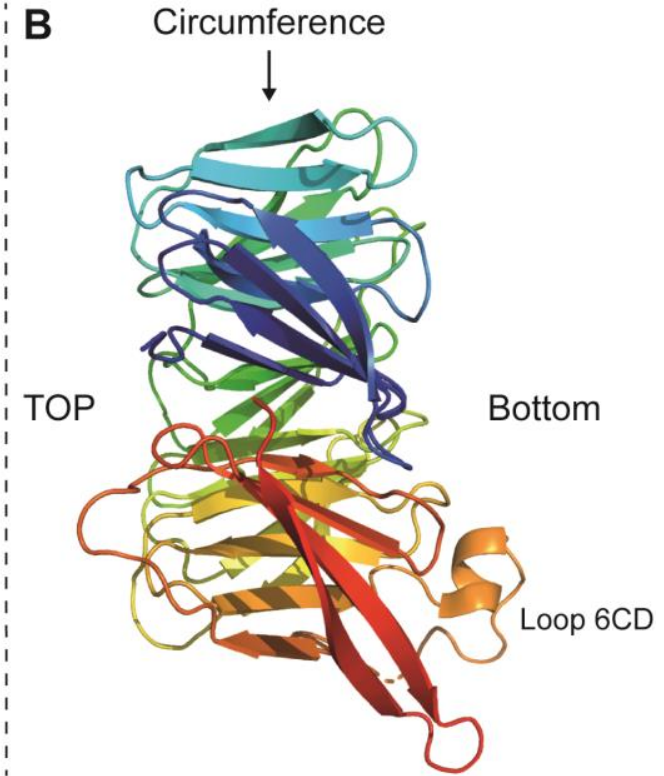

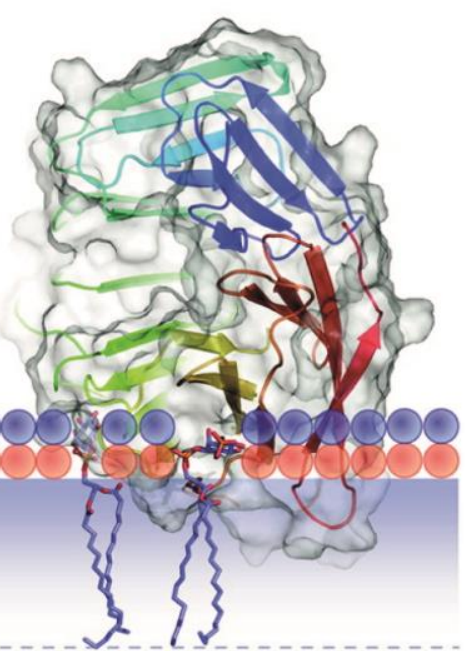

Figure 2.12: Structure and model for PI3P binding of KIHsv2

(A and B) Cartoon representation of the structure of KlHsv2 rainbow colored with blue to red from the $\mathrm{N}$ - to the C-terminus. KlHsv2 forms a seven-bladed $\beta$-propeller where each blade is composed of a four-stranded antiparallel $\beta$-sheet. The blades are numbered from one ( $\mathrm{N}$-terminus) to seven (C-terminus) while the single $\beta$-strands are termed $A$ to $D$ from the inner to the outer strand. Depicted is a view onto the bottom (A) and the circumference (B) of the propeller (modified from Krick et al. (2012)). (C) Model for the PI3P binding and membrane association of KlHsv2. The conserved FRRGmotif is part of two individual basic PI3P binding sites that act in concert. The $\beta$-propeller sits perpendicular on the membrane, As a result, the loops 6CD and 7CD insert into the membrane and support the association (modified from Thumm et al. (2013)).

PROPPINs are reported to preferentially bind PI3P and $\mathrm{PI}, 5 \mathrm{P}_{2}$ and thereby associate with membranes (Dove et al., 2004; Jeffries et al., 2004; Stromhaug et al., 2004; Polson et al., 2010). Interestingly, they do not contain any of the well characterized PI binding FYVE (Fab1, YOTB, Vac1 and EEA1), PH (pleckstrin homology) or PX (Phox homology) domains. Instead PI binding is mediated by a conserved FRRG-motif which is located on $\beta$-strand 5D and the connecting loop to 
$\beta$-strand 6A (Dove et al., 2004; Jeffries et al., 2004; Stromhaug et al., 2004; Kutateladze, 2010). The FRRG-motif is part of conserved, positively charged region which forms two basic pockets at the circumference of the $\beta$-propeller. Each of the two conserved arginine residues is oriented towards one of these pockets thereby forming two binding sites for PI3P or PI3,5P2 (Figure 2.12 C; Krick et al., 2012; Baskaran et al., 2012; Watanabe et al., 2012).

In the proposed model for membrane binding of PROPPINs, the two PI3P/PI3,5P2 binding sites are both required for an efficient membrane association. When bound to two $\mathrm{PI} 3 \mathrm{P} / \mathrm{PI} 3,5 \mathrm{P}_{2}$ molecules, the $\beta$-propeller sits perpendicular on the membrane and the loops 6CD and 7CD are inserted into the membrane (Figure 2.12 C). Especially loop 6CD was reported to act as an additional membrane anchor which supports the membrane association of PROPPINs by hydrophobic and electrostatic interactions (Krick et al., 2012; Baskaran et al., 2012; Watanabe et al., 2012; Busse et al., 2015).

\subsubsection{The functions of yeast PROPPINs}

The yeast PROPPINs Atg18, Atg21 and Hsv2 act as important PI3P/PI3,5P2 adaptors. PI3P, which represents about $30 \%$ of total PIs in yeast, and $\mathrm{PI} 3,5 \mathrm{P}_{2}$ are especially enriched at endosomes, the vacuole and the PAS making all three compartments potent places for PROPPIN recruitment (De Craene et al., 2017). Indeed, all three yeast PROPPINs localize PI3P-dependent to endosomes. The endosomal targeting of PROPPINs is regulated by the PI3-kinase complex II. The function of PROPPINs at endosomes however is still elusive (Stromhaug et al., 2004; Krick et al., 2008a). Furthermore, Atg18 and Atg21 localize PI3P-dependent to the PAS. The PAS localization, in contrast to the endosomal localization, is dependent on the PI3-kinase complex I (Reggiori et al., 2004; Suzuki et al., 2007; Krick et al., 2008a; Juris et al., 2015). Atg18 further shows a PI3,5P 2 -dependent vacuolar localization (Dove et al., 2004), whereas Atg21 only partially localizes to the vacuole and the vertices of vacuolar junctions. The main portion of Atg21 is associated with endosomes and the PAS (Stromhaug et al., 2004; Meiling-Wesse et al., 2004).

Atg18 belongs to the core autophagic machinery and is essential for all autophagic processes (Barth et al., 2001; Guan et al., 2001). Thereby, the PI3P binding activity 
of Atg18 is required for autophagosome biogenesis (Obara et al., 2008b). Furthermore, Atg18 forms a complex with Atg2 which is also a prerequisite for autophagy progression. Atg2 was recently found to have two membrane binding domains of which the C-terminal domain forms an amphipathic $\alpha$-helix and facilitates the binding of Atg18 to PI3P at the autophagic membrane (Kotani et al., 2018). This is in line with the finding that Atg2 independently binds to the PAS and is required for recruitment of Atg18 (Rieter et al., 2013). The interaction with Atg2 is mediated by the loops $2 \mathrm{BC}$ and $2 \mathrm{D}$ to $3 \mathrm{~A}$ of $\mathrm{Atg} 18$ which are located opposite to the conserved FRRG-motif (Watanabe et al., 2012; Rieter et al., 2013). The complex formation of Atg2 and Atg18 was reported to be independent of the PI3P binding activity of Atg18 (Obara et al., 2008b).

The Atg18-Atg2 complex further associates with Atg9 and participates in the recycling of Atg9-containing vesicles (Reggiori et al., 2004). The complex of Atg18, Atg2 and Atg9 localizes to the edges of the growing phagophore and was recently shown to form a functional contact site between the phagophore edges and the ER. The tethering of the ER membrane to the autophagic membrane seems to be mediated by the second N-terminal membrane binding domain of Atg2 (Suzuki et al., 2013; Graef et al., 2013; Gomez-Sanchez et al., 2018; Kotani et al., 2018).

At the vacuolar membrane, Atg18 is involved in additional non-autophagic functions. It affects retrograde membrane trafficking from the vacuole to the Golgi apparatus as well as vacuolar fragmentation by regulating the PI3P 5-kinase Fab1. Here, Atg18 does not seem to directly interact with Fab1 but with its regulator Vac7 and the scaffold protein Vac14 (Dove et al., 2004; Efe et al., 2007; Jin et al., 2008). Cells depleted for Atg18 display a defect in vacuolar fission and harbor significantly enlarged vacuoles (Dove et al., 2004).

In Pichia pastoris, the membrane association of Atg18 appears to be regulated by phosphorylation of loop 6CD (Tamura et al., 2013). Furthermore, in vitro studies revealed that upon membrane binding, the loop 6CD folds into an amphipathic $\alpha$-helix which inserts into the outer membrane leaflet. In addition, Atg18 was observed to oligomerize at the membrane which in combination with insertion of the amphipathic $\alpha$-helix is thought to be responsible for the observed tubulation and scission of GUVs. The formation of the amphipathic $\alpha$-helix and the resulting scission 
activity of Atg18 seems to be more important for its function in vacuolar fission as for autophagy (Scacioc et al., 2017; Gopaldass et al., 2017).

In contrast to Atg18, Atg21 only impairs but does not block unselective autophagy and is therefore not essential for its progression. However, it is strictly required for selective forms of autophagy including the Cvt-pathway, PMN and mitophagy (Barth et al., 2002; Meiling-Wesse et al., 2004; Krick et al., 2008b; Kanki et al., 2009a). Furthermore, Atg21 was reported to be essential for pexophagy in the yeasts Pichia angusta and Pichia pastoris (Leão-Helder et al., 2004; Tamura et al., 2014).

Atg21 is required for the recruitment of Atg8, Atg5 and Atg16 to the PAS as well as for the efficient lipidation of Atg8 (Stromhaug et al., 2004; Meiling-Wesse et al., 2004; Nair et al., 2010). A recent study revealed that Atg21 organizes the Atg8 lipidation at the phagophore by binding to PI3P and recruiting Atg8 as well as the E3-like Atg12 Atg5/Atg16 complex (Figure 2.13). The recruitment of the Atg8 lipidation complex is regulated by the interaction of Atg8 with the loop 2D to 3A at the top side of Atg21 and binding of Atg16 to the bottom side of Atg21. Interestingly, the interaction with Atg8 is not AIM-dependent but is mediated by the conserved F5K6-motif in the N-terminal arm-like domain of Atg8. The interaction with Atg16 depends on the two negatively charged residues D101 and E102 of Atg16 (Juris et al., 2015).

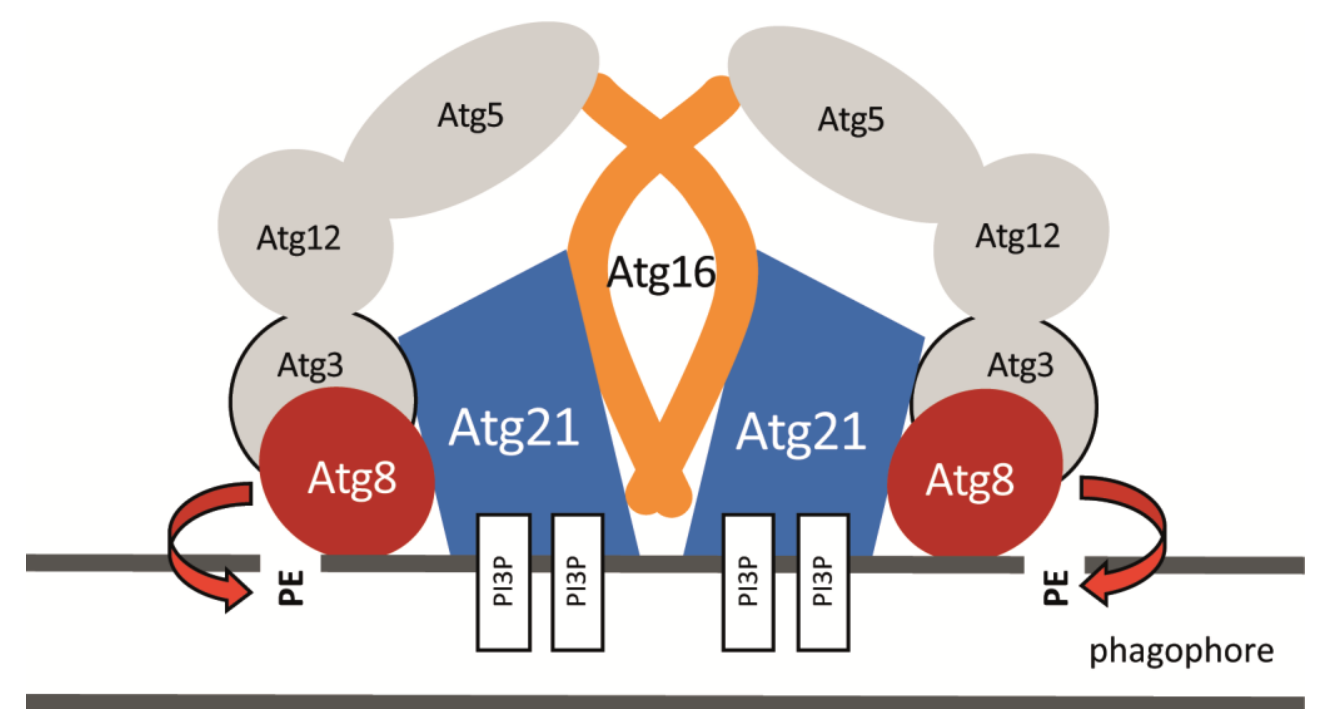

Figure 2.13: Model for the organization of the Atg8 lipidation by Atg21 at the autophagic membrane (Krick and Thumm, 2016)

In this model, Atg21 binds PI3P at the phagophore and thereby determines the site of Atg8-PE formation. It recruits the Atg8 Atg3 conjugate by binding to the conserved F5K6-motif in the Nterminal arm-like domain of Atg8. The interaction with Atg8 is mediated by loop 2D to 3A on the top 
side of Atg21. At its bottom side, Atg21 recruits the E3-like Atg12 Atg5/Atg16 complex by interacting with the two negatively charged residues D101 and E102 of Atg16. The dimeric CCD of Atg16 is thought to bind two Atg21 molecules which enhances the association with the autophagic membrane. By arranging the Atg8 lipidation complex at the autophagic membrane, Atg21 positions the Atg8 Atg3 conjugate close to PE and enhances the lipidation reaction.

Although Atg21 has no orthologue in mammals, the splice variant WIPI2b was reported to exhibit a similar function in the conjugation of LC3 to PE. WIPI2b directly interacts with Atg16L1, a human orthologue of Atg16, and thereby recruits the Atg12 Atg5/Atg16L1 complex to the PI3P-positive omegasome (Dooley et al., 2014).

Among all yeast PROPPINs, Hsv2 is the one that is functionally least characterized. It was named homolog of Svp1 2 (Hsv2) whereby Svp1 is another name for Atg18. So far, the only known function of Hsv2 is a partial impairment of PMN progression suggesting that Hsv2 like Atg21 could be a regulator of selective subtypes of autophagy (Dove et al., 2004; Krick et al., 2008a).

\subsection{Aim of this study}

PROPPINs are a family of important PI3P and PI3,5P 2 adaptors which are involved in autophagy and probably other vesicular transport pathways. Although they are structurally well characterized, their molecular function is still not fully understood. Human members of the PROPPIN-family are reported to be required for the autophagic degradation of pathogens in human cells, like WIPI2b in the clearance of Salmonella (Dooley et al., 2014; Proikas-Cezanne et al., 2015). Furthermore, mutations of WIPI4 are associated with human diseases, like the static encephalopathy of childhood with neurodegeneration in adulthood (SENDA) (Saitsu et al., 2013). WIPI4 is further reported to be overexpressed in pancreatic and kidney cancer cells (Proikas-Cezanne et al., 2004). Therefore, the elucidation of the molecular function of PROPPINs is also beneficial for the understanding of human diseases.

The overall aim of this study was a detailed analysis of the role of Atg21 in autophagy and other processes. Since the requirement of WIPI2b in the clearance of Salmonella is directly related to its ability to recruit the Atg12 Atg5/Atg16L1 complex (Dooley et al., 2014), one of the main goals of this study was the characterization of the molecular and dynamic details of the interaction between Atg21 and Atg16 (chap. 
4.1). Due to its interaction with components of the Atg8 lipidation complex, Atg21 is thought to determine the site of Atg8 lipidation (Juris et al., 2015). Therefore, a second goal of this study was the elucidation of the spatial distribution of Atg21 at the growing phagophore. Thereby, a novel contact site between vacuole and phagophore was identified and further characterized (chap. 4.2). Finally, a proximity-dependent assay was used to identify potential interaction partners of Atg21 by mass spectrometry (MS) analysis which could reveal further functions of Atg21 (chap. 4.3). 


\section{Materials and Methods}

\subsection{Materials}

Figures presented in this work were generated with the Adobe ${ }^{\circledR}$ Illustrator ${ }^{\circledR}$ CS6 software (Adobe Systems, San Jose, USA). Text editing and data processing was performed with Microsoft ${ }^{\circledR}$ Word and Excel 2016 (Microsoft, Redmond, USA).

\subsubsection{Yeast and bacteria strains}

\subsubsection{Saccharomyces cerevisiae strains}

Table 3.1: Saccharomyces cerevisiae strains used in this study

\begin{tabular}{|c|c|c|}
\hline Strain & Genotype & Reference \\
\hline WCG4a (WT) & MAT $\alpha$ his2-11,15 leu2-3,112 ura3 & (Thumm et al., 1994) \\
\hline $\operatorname{atg} 1 \Delta$ & WCG4a $\operatorname{atg} 1 \triangle:: K A N$ & (Straub et al., 1997) \\
\hline $\operatorname{atg} 8 \Delta$ & WCG4a $\operatorname{atg} 8 \triangle:: K A N$ & (Lang et al., 1998) \\
\hline $\operatorname{atg} 8 \Delta \operatorname{atg} 3 \Delta$ & WCG4a atg $8 \Delta:: K A N$ atg $3 \Delta:: h p h N T 1$ & This study \\
\hline atg8 $8 T G 14-3 \times G F P$ & $\begin{array}{l}\text { WCG4a atg8D::KAN } \\
\text { ATG14-3xGFP(S65T)::HIS3MX6 }\end{array}$ & This study \\
\hline $\operatorname{atg} 8 \Delta \operatorname{atg} 18 \Delta$ & WCG4a $\operatorname{atg} 8 \Delta:: K A N$ atg184::natNT2 & $\begin{array}{l}\text { L. Juris (AG Thumm, } \\
\text { University Göttingen) }\end{array}$ \\
\hline $\operatorname{atg} 8 \Delta \operatorname{atg} 21 \Delta$ & WCG4a $\operatorname{atg} 8 \Delta:: K A N$ atg214::natNT2 & $\begin{array}{l}\text { L. Juris (AG Thumm, } \\
\text { University Göttingen) }\end{array}$ \\
\hline $\operatorname{atg} 8 \Delta \operatorname{atg} 21 \Delta \operatorname{atg} 16 \Delta$ & $\begin{array}{l}\text { WCG4a } \operatorname{atg} 8 \Delta:: K A N \text { atg } 21 \Delta:: h p h N T 1 \\
\operatorname{atg} 16 \Delta:: \text { natNT2 }\end{array}$ & (Juris et al., 2015) \\
\hline $\begin{array}{l}\operatorname{atg} 8 \Delta \operatorname{atg} 21 \Delta \\
\text { VPH1-mCherry }\end{array}$ & $\begin{array}{l}\text { WCG4a atg8D::KAN atg214::natNT2 } \\
\text { VPH1-mCherry::hphNT1 }\end{array}$ & This study \\
\hline $\operatorname{atg} 8 \triangle A P E 1-R F P$ & $\begin{array}{l}\text { WCG4a } \text { atg } 8 \Delta:: K A N \\
\text { APE1-mRFP::hphNT1 }\end{array}$ & This study \\
\hline $\begin{array}{l}\operatorname{atg} 8 \triangle A P E 1-R F P \\
3 x \operatorname{tag} B F P-P H O 8\end{array}$ & $\begin{array}{l}\text { WCG4a } \operatorname{atg} 8 \Delta:: K A N \\
\text { APE1-mRFP::hphNT1 pRS305- } P_{P G K 1^{-}} \\
\text {3xtagBFP-PHO8 }\end{array}$ & This study \\
\hline $\begin{array}{l}\operatorname{atg} 8 \triangle A P E 1-R F P \\
\operatorname{atg} 21 \triangle\end{array}$ & $\begin{array}{l}\text { WCG4a atg } 8 \Delta:: K A N \\
\text { APE1-mRFP::hphNT1 atg21 }:: \text { natNT2 }\end{array}$ & This study \\
\hline $\begin{array}{l}\operatorname{atg} 8 \triangle A P E 1-R F P \\
\operatorname{atg} 21 \triangle 3 x \operatorname{tag} B F P- \\
\text { PHO8 }\end{array}$ & 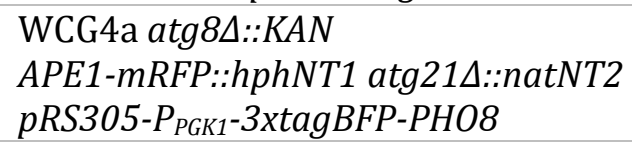 & This study \\
\hline $\begin{array}{l}\operatorname{atg} 8 \triangle A P E 1-R F P \\
\text { vac8 }\end{array}$ & $\begin{array}{l}\text { WCG4a atg } 8 \Delta:: K A N \\
\text { APE1-mRFP::hphNT1 vac8D::natNT2 }\end{array}$ & This study \\
\hline $\begin{array}{l}\operatorname{atg} 8 \triangle A P E 1-R F P \\
\text { vac8D 3xtagBFP- } \\
\text { PHO8 }\end{array}$ & $\begin{array}{l}\text { WCG4a atg } 8 \Delta:: K A N \\
\text { APE1-mRFP::hphNT1 vac8D::natNT2 } \\
\text { pRS305-PPGK1-3xtagBFP-PHO8 }\end{array}$ & This study \\
\hline $\operatorname{atg} 8 \Delta$ vac8 & WCG4a atg $8 \Delta:: K A N$ vac8D::hphNT1 & This study \\
\hline $\operatorname{atg} 8 \triangle V A C 8-3 \times G F P$ & $\begin{array}{l}\text { WCG4a atg } 8 \Delta:: K A N \text { VAC8- } \\
3 x G F P(S 65 T):: H I S 3 M X 6\end{array}$ & This study \\
\hline
\end{tabular}




\begin{tabular}{|c|c|c|}
\hline $\begin{array}{l}\operatorname{atg} 8 \Delta 3 x \operatorname{tag} B F P- \\
\text { PHO8 }\end{array}$ & $\begin{array}{l}\text { WCG4a atg8D::KAN pRS305- } P_{P G K 1^{-}} \\
\text {3xtagBFP-PHO8 LEU2::natNT2 }\end{array}$ & $\begin{array}{l}\text { F. Otto (AG Thumm, } \\
\text { University Göttingen) }\end{array}$ \\
\hline atg8D VPH1-GFP & $\begin{array}{l}\text { WCG4a atg8D::KAN } \\
\text { VPH1-yeGFP::hphNT1 }\end{array}$ & This study \\
\hline $\operatorname{atg} 16 \Delta$ & WCG4a atg164::natNT2 & $\begin{array}{l}\text { L. Juris (AG Thumm, } \\
\text { University Göttingen) }\end{array}$ \\
\hline $\operatorname{atg} 16 \triangle A P E 1-R F P$ & $\begin{array}{l}\text { WCG4a atg164::natNT2 } \\
\text { APE1-RFP::hphNT1 }\end{array}$ & This study \\
\hline $\operatorname{atg} 21 \Delta$ & WCG4a $\operatorname{atg} 21 \triangle:: K A N$ & (Barth et al., 2002) \\
\hline $\operatorname{atg} 21 \Delta \arg 4 \Delta \operatorname{lys} 1 \Delta$ & $\begin{array}{l}\text { WCG4a } \operatorname{atg} 21 \Delta:: K A N \text { arg } 4 \Delta:: h p h N T 1 \\
\text { lys1A::natNT2 }\end{array}$ & This study \\
\hline $\operatorname{atg} 21 \Delta \operatorname{atg} 16 \Delta$ & 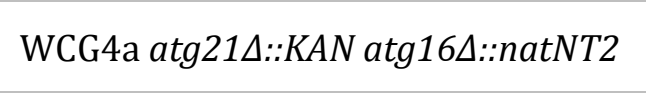 & $\begin{array}{l}\text { R. Krick (AG Thumm, } \\
\text { University Göttingen) }\end{array}$ \\
\hline $\operatorname{atg} 21 \Delta \operatorname{atg} 16 \Delta \operatorname{atg} 5 \Delta$ & $\begin{array}{l}\text { WCG } 4 \text { a } \operatorname{atg} 21 \Delta:: K A N \text { atg } 16 \Delta:: n a t N T 2 \\
\operatorname{atg} 5 \Delta:: h p h N T 1\end{array}$ & This study \\
\hline $\operatorname{atg} 21 \triangle V A C 8-6 \times H A$ & $\begin{array}{l}\text { WCG4a atg21 }:: K A N \\
\text { VAC8-6xHA::hphNT1 }\end{array}$ & This study \\
\hline SEY 6210 (SEY WT) & $\begin{array}{l}\text { Sey } 6210 \text { MATa ura3-52 leu2-3,112 } \\
\text { his3- } \triangle 200 \text { lys 2-801 trp1- } \Delta 901 \text { suc2- } \Delta 9 \\
\text { mel GAL }\end{array}$ & (Harding et al., 1996) \\
\hline $\operatorname{atg} 16 \Delta$ & Sey 6210 atg164::natNT2 & (Juris et al., 2015) \\
\hline $\operatorname{atg} 21 \Delta \operatorname{atg} 16 \Delta$ & $\begin{array}{l}\text { Sey } 6210 \text { atg16 } 16: \text { natNT2 } \\
\operatorname{atg} 21 \Delta:: h p h N T 1\end{array}$ & This study \\
\hline
\end{tabular}

\subsubsection{Escherichia coli strains}

Table 3.2: Escherichia coli strains used in this study

\begin{tabular}{|c|c|c|}
\hline Strain & Genotype & Reference \\
\hline $\mathrm{NEB}^{\circledR}$ 5-alpha & $\begin{array}{l}\text { fhuA2 } \triangle \text { (argF-lacZ)U169 phoA glnV44 } \\
\text { \$80د (lacZ)M15 gyrA96 recA1 relA1 } \\
\text { endA1 thi-1 hsdR17 }\end{array}$ & $\begin{array}{l}\text { New England Biolabs } \\
\text { (Frankfurt) }\end{array}$ \\
\hline XL1-Blue & $\begin{array}{l}\text { recA1 endA1 gyrA96 thi-1 hsdR17 supE44 } \\
\text { relA1 lac [F' proAB lacIqZDM15 Tn10 } \\
\left.\left(\text { Tet }^{r}\right)\right]\end{array}$ & Agilent (Santa Clara, USA) \\
\hline XL10-Gold & $\begin{array}{l}\text { Tetr } \Delta(\text { mcrA }) 183 \Delta(\text { mcrCB-hsdSMR- } \\
\text { mrr)173 endA1 supE44 thi-1 recA1 } \\
\text { gyrA96 relA1 lac Hte [F proAB } \\
\left.\text { lacI }{ }^{\prime} Z \Delta M 15 \text { Tn10(Tetr) Amy } \text { Cam }^{r}\right]\end{array}$ & Agilent (Santa Clara, USA) \\
\hline
\end{tabular}

\subsubsection{Plasmids}

Table 3.3: Plasmids used for molecular cloning and gene expression in this study

\begin{tabular}{|c|c|c|}
\hline Name & Genotype & Reference \\
\hline Atg3-GFP & $\begin{array}{l}\text { pRS313 } P_{A T G 3}-A T G 3-D 265_{A T G 3}-y e G F P- \\
\text { G266 }\end{array}$ & $\begin{array}{l}\text { P. Schlotterhose } \\
\text { (AG Thumm, } \\
\text { University Göttingen) }\end{array}$ \\
\hline Atg3 $3^{\text {AIM-GFP }}$ & 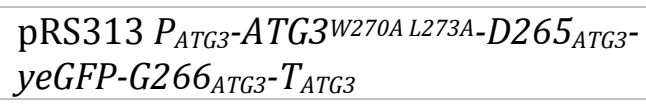 & This study \\
\hline
\end{tabular}




\begin{tabular}{|c|c|c|}
\hline Atg3 ${ }^{C 234 A}-G F P$ & 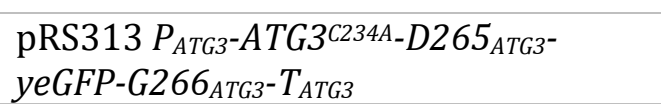 & This study \\
\hline Atg5-2xFLAG & pRS313 $P_{C U P 1}-A T G 5-2 x F L A G$ & This study \\
\hline Atg16-BirA* & 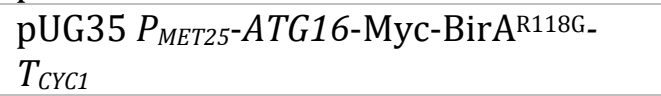 & This study \\
\hline $\operatorname{Atg} 16-C_{u b}$ & pRS313 $P_{\text {MET25-ATG16- }} C_{u b}-R U R A 3$ & (Juris et al., 2015) \\
\hline Atg16-HA & pRS313 $P_{C U P 1}-A T G 16-6 \times \mathrm{xA}$ & (Juris et al., 2015) \\
\hline Atg16 ${ }^{\text {D101R-HA }}$ & pRS313 $P_{C U P 1}-A T G 16^{D 101 R_{-}} 6 \mathrm{xHA}$ & This study \\
\hline Atg16D101R E102R-HA & pRS313 $P_{C U P 1}-A T G 16$ D101R E102R-6xHA & This study \\
\hline Atg16 E102R-HA & pRS313 $P_{C U P 1}-A T G 16^{E 102 R_{-}} 6 \mathrm{xHA}$ & This study \\
\hline Atg16E81R-HA & pRS313 $P_{C U P 1}-A T G 16^{E 81 R-6 x H A}$ & This study \\
\hline Atg16 $1678 \mathrm{E}-\mathrm{HA}$ & pRS313 $P_{C U P 1}-A T G 16^{K 78 E-6 x H A}$ & This study \\
\hline Atg16 $1694 \mathrm{~A}-\mathrm{HA}$ & pRS313 $P_{\text {CUP1-ATG16 }}{ }^{\text {K94A-6xHA }}$ & (Juris, 2014) \\
\hline Atg16 ${ }^{\text {K94E-HA }}$ & pRS313 $P_{C U P 1}-A T G 16^{K^{K 94 E}-6 x H A}$ & This study \\
\hline Atg16-GFP & pRS313 $P_{A T G 16}-A T G 16-G F P$ & (Juris et al., 2015) \\
\hline Atg16 D101R-GFP & pRS313 $P_{A T G 16}-A T G 16^{D 101 R}$-GFP & This study \\
\hline $\operatorname{Atg} 16^{\mathrm{K} 94 \mathrm{E}-\mathrm{GFP}}$ & pRS313 $P_{A T G 16-A T G 16}{ }^{K 94 E_{-}}$-GFP & This study \\
\hline Atg18-GFP & pUG23 $P_{A T G 18}-A T G 18$-yeGFP- $T_{C Y C 1}$ & This study \\
\hline Atg21-BirA* & 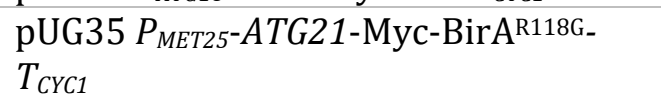 & This study \\
\hline Atg21 C-YC & $\begin{array}{l}\text { pRS426 } 2 \mu \text { Amp }{ }^{\mathrm{R}} \text { ori lacZ' URA3 } P_{A D H 1^{-}} \\
\text {ATG21-C-YC-T }\end{array}$ & $\begin{array}{l}\text { L. Juris (AG Thumm, } \\
\text { University Göttingen) }\end{array}$ \\
\hline Atg21-HA & 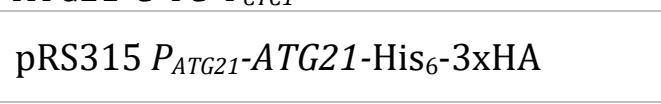 & $\begin{array}{l}\text { (AG Thumm, } \\
\text { University Göttingen) }\end{array}$ \\
\hline Atg21 $1^{\text {FTTG-HA }}$ & 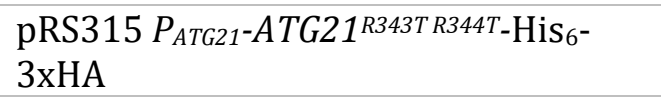 & This study \\
\hline Atg21-YFP & pRS313 $P_{A T G 21}-A T G 21-E Y F P$ & This study \\
\hline Atg21-YFP & pRS315 $P_{A T G 21}-A T G 21$-EYFP & This study \\
\hline Atg21-YFP & pRS316 $P_{A T G 21}-A T G 21$-EYFP & $\begin{array}{l}\text { L. Juris (AG Thumm, } \\
\text { University Göttingen) }\end{array}$ \\
\hline BirA* $^{*}$ & pUG36 $P_{M E T 25}-$ Myc-BirA ${ }^{\text {R118G- }} T_{C Y C 1}$ & This study \\
\hline BirA*-Atg16 & $\begin{array}{l}\text { pUG36 } P_{M E T 25}-\mathrm{Myc}-\mathrm{BirA}^{\mathrm{R} 118 \mathrm{G}-A T G 16-} \\
T_{C Y C 1}\end{array}$ & This study \\
\hline BirA*-Atg21 & $\begin{array}{l}\text { pUG36 } P_{M E T 25} \text {-Myc-BirAR118G-ATG21- } \\
T_{C Y C 1}\end{array}$ & This study \\
\hline BirA*-Atg21 ${ }^{\text {FTTG }}$ & $\begin{array}{l}\text { pUG36 } P_{M E T 25} \text {-Myc-BirAR118G-ATG21 R343T } \\
\text { R344T- } T_{C Y C 1}\end{array}$ & This study \\
\hline CUP1-pApe1 & $\begin{array}{l}\mathrm{pRS} 4232 \mu \mathrm{Amp}^{\mathrm{R}} \text { ori lacZ' HIS3 } P_{C U P 1^{-}} \\
\text {APE1 }\end{array}$ & $\begin{array}{l}\text { P. Schlotterhose } \\
\text { (AG Thumm, } \\
\text { University Göttingen) }\end{array}$ \\
\hline CUP1-pApe1 & pYEX-BX $2 \mu$ Amp ${ }^{\mathrm{R}}$ ori $U R A 3 P_{C U P 1}-A P E 1$ & (Suzuki et al., 2013) \\
\hline GFP-Atg8 & pRS313 $P_{A T G 8}$-GFP-ATG8- $T_{A T G 8}$ & $\begin{array}{l}\text { (AG Thumm, } \\
\text { University Göttingen) }\end{array}$ \\
\hline GFP-Atg21 & pUG36 $P_{M E T 25}$-yeGFP-ATG21- $T_{C Y C 1}$ & (Krick et al., 2008a) \\
\hline GFP-Atg21D9R & pUG36 $P_{M E T 25-y e G F P-A T G 211^{D 9 R}-T_{C Y C 1}}$ & This study \\
\hline GFP-Atg21 D28R & pUG36 $P_{M E T 25}$-yeGFP-ATG21 ${ }^{D 28 R-} T_{C Y C 1}$ & This study \\
\hline GFP-Atg21 D167R & pUG36 $P_{M E T 25}$-yeGFP-ATG21 ${ }^{D 167 R-} T_{C Y C 1}$ & This study \\
\hline GFP-Atg21 ${ }^{\mathrm{E} 137 \mathrm{R}}$ & pUG36 $P_{M E T 25}$-yeGFP-ATG21 $1^{E 137 R_{-}} T_{C Y C 1}$ & This study \\
\hline GFP-Atg21FTTG & $\begin{array}{l}\text { pUG36 } P_{M E T 25} \text {-yeGFP-ATG21 R343T R344T- } \\
T_{C Y C 1}\end{array}$ & $\begin{array}{l}\text { R. Krick (AG Thumm, } \\
\text { University Göttingen) }\end{array}$ \\
\hline GFP-Atg21 $1{ }^{\mathrm{K} 130 \mathrm{E}}$ & pUG36 $P_{M E T 25}$-yeGFP-ATG21 ${ }^{\text {K130E- }} T_{C Y C 1}$ & This study \\
\hline GFP-Atg21 K152E & pUG36 $P_{M E T 25}$-yeGFP-ATG21 ${ }^{\text {K152E- }} T_{C Y C 1}$ & This study \\
\hline
\end{tabular}




\begin{tabular}{|c|c|c|}
\hline GFP-Atg21 ${ }^{\mathrm{K} 172 \mathrm{E}}$ & pUG36 $P_{\text {MET25-yeGFP-ATG21 }}{ }^{\text {K172E- }} T_{C Y C 1}$ & This study \\
\hline GFP-Atg21 R151E & pUG36 $P_{\text {MET25-yeGFP-ATG21 }}{ }^{\text {R151E- }} T_{C Y C 1}$ & This study \\
\hline GFP-Atg21 R151E K152E & $\begin{array}{l}\text { pUG36 } P_{\text {MET25-yeGFP-ATG21 }}{ }^{\text {R151E K152E- }} \\
T_{C Y C 1}\end{array}$ & This study \\
\hline GFP-Atg21 S105A T106A & $\begin{array}{l}\text { pUG36 } P_{M E T 25} \text {-yeGFP-ATG21 }{ }^{\text {S105A T106A- }} \\
T_{C Y C 1}\end{array}$ & This study \\
\hline GST-Atg16 & $\begin{array}{l}\text { pGEX-4T-3 Amp }{ }^{\mathrm{R}} \text { ori lacI tacP-GST- } \\
\text { Atg16 }\end{array}$ & (Juris et al., 2015) \\
\hline mCherry-Atg5 & pUG36 $P_{M E T 25}$-mCherry-ATG5- $T_{C Y C 1}$ & This study \\
\hline mCherry-Atg8 & pRS315 $P_{A T G 8}$-mCherry-ATG8- $T_{A T G 8}$ & $\begin{array}{l}\text { F. Otto (AG Thumm, } \\
\text { University Göttingen) }\end{array}$ \\
\hline mCherry-Atg21 & pUG36 $P_{M E T 25}$-mCherry-ATG21- $T_{C Y C 1}$ & (Juris et al., 2015) \\
\hline mCherry-Atg21 D28R & pUG36 $P_{M E T 25}-\mathrm{mCherry-ATG211^{D28R } - T _ { C Y C 1 }}$ & This study \\
\hline mCherry-Atg21 FTTG & $\begin{array}{l}\text { pUG36 } P_{M E T 25} \text {-mCherry-ATG21 } \\
T_{C Y C 1}{ }_{\text {R } 343 T ~ R 344 T_{-}}\end{array}$ & (Juris et al., 2015) \\
\hline mCherry-Atg21 & pUG36 $P_{M E T 25}-m$ Cherry-ATG21 ${ }^{\text {K130E- }} T_{C Y C 1}$ & This study \\
\hline mCherry-Atg21 R151E & pUG36 $P_{\text {MET25-mCherry-ATG21 }}{ }^{R 151 E-} T_{C Y C 1}$ & This study \\
\hline mRFP-2xFYVE & pRS315 $P_{T E F}-2 x F Y V E(H r s)-T_{C Y C 1}$ & $\begin{array}{l}\text { (AG Thumm, } \\
\text { University Göttingen) }\end{array}$ \\
\hline yomTagBFP2-Atg8 & pRS313 $P_{A T G 8-y o m T a g B F P 2-A T G 8-T_{A T G 8}}$ & $\begin{array}{l}\text { F. Otto (AG Thumm, } \\
\text { University Göttingen) }\end{array}$ \\
\hline $\mathrm{N}_{\mathrm{ub}}-\operatorname{Atg} 5$ & pRS314 $P_{C U P 1}-N_{u b}-A T G 5-T_{A T G 5}$ & (Juris et al., 2015) \\
\hline $\mathrm{N}_{\mathrm{ub}}-\operatorname{Atg} 21$ & pRS314 $P_{C U P 1}-N_{u b}-A T G 21$ & $\begin{array}{l}\text { F. Reggiori (University } \\
\text { of Groningen) }\end{array}$ \\
\hline $\mathrm{N}_{\mathrm{ub}}-\operatorname{Atg} 21^{\mathrm{FTTG}}$ & pRS314 $P_{C U P 1}-N_{u b}-A T G 21$ R343T R344T & This study \\
\hline $\mathrm{N}_{\mathrm{ub}}-\mathrm{Ubc} 6$ & pRS314 $P_{C U P 1}-N_{u b}-U B C 6$ & $\begin{array}{l}\text { F. Reggiori (University } \\
\text { of Groningen) }\end{array}$ \\
\hline $\begin{array}{l}\text { pFA6a-Myc- } \\
\text { BirA*_TRP1 }\end{array}$ & $\mathrm{Amp}^{\mathrm{R}}$ ori $T R P 1 \mathrm{Myc}-\mathrm{Bir} \mathrm{A}^{\mathrm{R} 118 \mathrm{G}}$ & $\begin{array}{l}\text { H. D. Schmitt (Dept. of } \\
\text { Neurobiology; MPI for } \\
\text { Biophysical Chemistry } \\
\text { Göttingen) }\end{array}$ \\
\hline pRS313 & CEN/ARS Amp ${ }^{\mathrm{R}}$ ori lacZ' HIS3 & $\begin{array}{l}\text { (Sikorski and Hieter, } \\
\text { 1989) }\end{array}$ \\
\hline pRS313-P $\mathrm{P}_{\text {CuP1 }}$ & pRS313 $P_{C U P 1}-$ lacZ'/MCS & $\begin{array}{l}\text { R. Krick (AG Thumm, } \\
\text { University Göttingen) }\end{array}$ \\
\hline pRS314 & CEN/ARS Amp ${ }^{\mathrm{R}}$ ori lacZ' TRP1 & $\begin{array}{l}\text { (Sikorski and Hieter, } \\
\text { 1989) }\end{array}$ \\
\hline pRS315 & CEN/ARS Amp R ori lacZ' LEU2 & $\begin{array}{l}\text { (Sikorski and Hieter, } \\
\text { 1989) }\end{array}$ \\
\hline pRS316 & CEN/ARS Amp ${ }^{\mathrm{R}}$ ori lacZ' URA3 & $\begin{array}{l}\text { (Sikorski and Hieter, } \\
\text { 1989) }\end{array}$ \\
\hline pRS416-MET25 & $\begin{array}{l}\text { CEN/ARS Amp }{ }^{\mathrm{R}} \text { ori } U R A 3 P_{M_{E T 25^{-}}} \\
\text {lacZ'/MCS-T }_{C Y C 1}\end{array}$ & (Mumberg et al., 1994) \\
\hline pUG23 & $\begin{array}{l}\text { CEN/ARS Amp }{ }^{\mathrm{R}} \text { ori } H I S 3 P_{M E T 25^{-}} \\
\text {lacZ'/MCS-yeGFP-T }_{C Y C 1}\end{array}$ & $\begin{array}{l}\text { (Niedenthal et al., } \\
\text { 1996) }\end{array}$ \\
\hline pUG35 & $\begin{array}{l}\text { CEN/ARS Amp }{ }^{\mathrm{R}} \text { ori } U R A 3 P_{M E T 25^{-}} \\
\text {lacZ'/MCS-yeGFP- } T_{C Y C 1}\end{array}$ & $\begin{array}{l}\text { (Niedenthal et al., } \\
\text { 1996) }\end{array}$ \\
\hline $\begin{array}{l}\text { pUG35-MCS-Myc- } \\
\text { BirA* }\end{array}$ & $\begin{array}{l}\text { CEN/ARS Amp }{ }^{\mathrm{R}} \text { ori } U R A 3 P_{M E T 25^{-}} \\
\text {lacZ'/MCS-Myc-BirA } 118 \mathrm{G}-T_{C Y C 1}\end{array}$ & This study \\
\hline pUG36 & 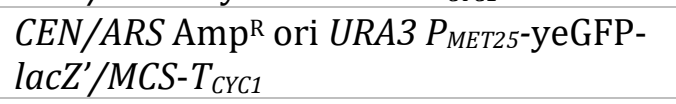 & $\begin{array}{l}\text { (Niedenthal et al., } \\
\text { 1996) }\end{array}$ \\
\hline pUG36-mCherry & $\begin{array}{l}\text { CEN/ARS Amp }{ }^{\mathrm{R}} \text { ori } U R A 3 P_{M_{\text {MET25- }}} \\
\text { mCherry-lacZ'/MCS- } T_{C Y C 1}\end{array}$ & $\begin{array}{l}\text { R. Krick (AG Thumm, } \\
\text { University Göttingen) }\end{array}$ \\
\hline
\end{tabular}




\begin{tabular}{|c|c|c|}
\hline $\begin{array}{l}\text { pUG36-Myc-BirA*- } \\
\text { MCS }\end{array}$ & 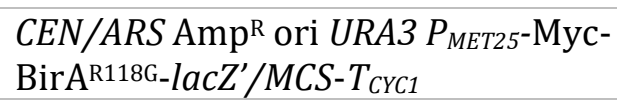 & This study \\
\hline Sec63-GFP & 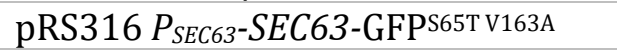 & (Prinz et al., 2000) \\
\hline Ste14- $\mathrm{C}_{\mathrm{ub}}$ & pRS313 $P_{\text {MET25-STE14- }} C_{u b}-R U R A 3$ & $\begin{array}{l}\text { F. Reggiori (University } \\
\text { of Groningen) }\end{array}$ \\
\hline Vac8-GFP & pUG23 $P_{V A C 8}$-VAC8-yeGFP- $T_{C Y C 1}$ & This study \\
\hline
\end{tabular}

Table 3.4: Plasmids used to amplify selection cassettes for gene deletions and chromosomal tagging in this study

\begin{tabular}{|c|c|c|}
\hline Name & Genotype & Reference \\
\hline $\begin{array}{l}\text { pFA6a-3xGFP(S65T)- } \\
\text { HIS3MX6 }\end{array}$ & $\begin{array}{l}\text { Amp }^{\mathrm{R}} \text { ori } 3 \times \mathrm{xGFP} 65 \mathrm{~T}-T_{A D H 1} P_{T E F^{-}} \\
\text {HIS3MX6-T }\end{array}$ & $\begin{array}{l}\text { L. Marquardt (AG Thumm, } \\
\text { University Göttingen) }\end{array}$ \\
\hline pFA6a-hphNT1 & $\mathrm{Amp}^{\mathrm{R}}$ ori $P_{T E F}-\mathrm{Hyg}^{\mathrm{R}}-T_{C Y C 1}$ & $\begin{array}{l}\text { Euroscarf; (Janke et al., } \\
\text { 2004) }\end{array}$ \\
\hline pFA6a-natNT2 & Amp ${ }^{\mathrm{R}}$ ori $P_{T E F}-\mathrm{Nrs}{ }^{\mathrm{R}}-T_{A D H 1}$ & $\begin{array}{l}\text { Euroscarf; (Janke et al., } \\
\text { 2004) }\end{array}$ \\
\hline pYM16 & $\mathrm{Amp}^{\mathrm{R}}$ ori $6 \mathrm{xHA} P_{T E F}-\mathrm{Hyg}-T_{C Y C 1}$ & $\begin{array}{l}\text { Euroscarf; (Janke et al., } \\
\text { 2004) }\end{array}$ \\
\hline pYM25 & Amp ${ }^{\mathrm{R}}$ ori yeGFP $P_{T E F}-\mathrm{Hyg} g^{\mathrm{R}}-T_{C Y C 1}$ & $\begin{array}{l}\text { Euroscarf; (Janke et al., } \\
\text { 2004) }\end{array}$ \\
\hline pYM25-mCherry & Amp ${ }^{\mathrm{R}}$ ori mCherry $P_{T E F}-\mathrm{Hyg}^{\mathrm{R}}-T_{C Y C 1}$ & $\begin{array}{l}\text { S. Karnebeck (AG Thumm, } \\
\text { University Göttingen) }\end{array}$ \\
\hline pYM25-RFP & $\mathrm{Amp}^{\mathrm{R}}$ ori mRFP1 $P_{T E F}-\mathrm{Hyg}^{\mathrm{R}}-T_{C Y C 1}$ & $\begin{array}{l}\text { L. Marquardt (AG Thumm, } \\
\text { University Göttingen) }\end{array}$ \\
\hline $\begin{array}{l}\text { pRS305-P } \text { PGK1- }^{-} \\
\text {3xtagBFP-PHO8 }\end{array}$ & $\begin{array}{l}\text { Amp }^{\mathrm{R}} \text { ori } P_{P G K 1}-3 x \operatorname{tagBFP}-\mathrm{PHO8} \\
\text { LEU2 }\end{array}$ & (Graef et al., 2013) \\
\hline
\end{tabular}

\subsubsection{Oligonucleotides}

The used deoxyoligonucleotides were designed with the help of the Gene Construction Kit ${ }^{\circledR} 4.0$ program (Textco BioSoftware, Raleigh, USA) or the Snap Gene software (GSL Biotech LLC, Chicago, USA) and synthesized by Eurofins Genomics GmbH (Ebersberg).

Table 3.5: Oligonucleotides for cloning and sequencing used in this study

\begin{tabular}{ll}
\hline Name & Sequence \\
\hline atg21_Seq1 for & GTTTTCCCACATGAAATTGTTG \\
\hline atg21_seq1 rev & CAACAATTTCATGTGGGAAAAC \\
\hline atg21_seq3 for & GAAATACCTCTCTCGAAACC \\
\hline atg21-seq3 rev & GGTTTCGAGAGAGGTATTTC \\
\hline atg21-seq4 for & GAATCACGGAATAATGAGGAG \\
\hline atg21_seq5 for & GATGGTAAACTGCTTGCTAC \\
\hline ATG16 seq 1 for & GCTACTACACTTGCTCGC \\
\hline Atg16_Seq2 for & GGAGCAAGAAATACGAAG \\
\hline Atg16 Seq2 rev & CTTCGTATTTCTTGCTCC \\
\hline Atg16 Seq3 for & GCCATGAACAGCGAAATAG \\
\hline Atg16 Seq3 rev & CTATTTCGCTGTTCATGGC \\
\hline
\end{tabular}




\begin{tabular}{|c|c|}
\hline ATG16 seq 4 rev & GTGAGTTATAGTTGTATTCC \\
\hline Atg5_Seq1 for & CСССТCATTTGGAACAAG \\
\hline Atg5_Seq1 rev & CTTGTTCCAAATGAGGGG \\
\hline Atg5_Seq2 for & GTTCATCAAGACCGCGAC \\
\hline Atg5_Seq2 rev & GTCGCGGTCTTGATGAAC \\
\hline Ape1_seq1 for & CGTGGTGCATCCTCGAGCAC \\
\hline Ape1_seq1 rev & GTGCTCGAGGATGCACCACG \\
\hline Ape1_seq2 for & GCACCACGTCTAGATGACAGG \\
\hline Ape1_seq3 for & GCACAGTTGTCCATGCACAGC \\
\hline TApe1_seq rev & GGGAGCCGCGCGTTACCCGG \\
\hline Atg18-seq0 for & CTCAGCAGTGGTCATTGCCTTCG \\
\hline Atg18-seq2 rev & CTGTTGGCTACCGAAGGAGAC \\
\hline Atg18-seq3 for & GACATTGAAACGGGTGATAAG \\
\hline Atg18-seq4 rev & GGAATTGGTTTCAACGGGAAG \\
\hline PVac8_seq1 for & CCTACGAAGACAGGAACTGAG \\
\hline Vac8_seq1 rev & GGTGAGACACTGGCCTCGTC \\
\hline Vac8_seq2 for & CGTCCACCGATCCTGATGTTC \\
\hline Vac8_seq3 for & GGCAGATGTGACCTTTGAGCG \\
\hline Atg3_Seq1 for & GGTAACGCCAGGGTTTTCCC \\
\hline Atg3_Seq2 for & СTCCTGAGGAGTTTGTACAAGC \\
\hline Atg3_Seq3 for & GCGCAAGAAAGGTATTACGACC \\
\hline Atg3_Seq5 for & GTTGTGTGGAATTGTGAGCGG \\
\hline Seq MET 25 for & TGAAAACTGTGTAACATAAT \\
\hline pUG 36-GFP1 for & CAATTGGTGATGGTCCAGTC \\
\hline GFP seq for & GGTTCTGTTCAATTAGCTGAC \\
\hline pUG36 rev & GGGACCTAGACTTCAGGTTG \\
\hline Cherry seq for & GGCGCCTACAACGTCAACATC \\
\hline pPS313-CUP1 for & GCAATATGGATTGTCAGAATC \\
\hline pPS313-CUP1 rev & CACACAGGAAACAGCTATGACC \\
\hline CUP1_seq rev & GATTCTGACAATCCATATTGC \\
\hline BirA_seq for & GGATTGGCACCTTATCTGTCG \\
\hline Prs316seq for & GATGTGCTGCAAGGCGATTAAG \\
\hline N_YC Seq for & GTGCAGCTCGCCGACCAC \\
\hline C-YC Seq rev & CTCGTCCATGCCGAGAGTG \\
\hline hphNT1 rev & CAATCGCGCATATGAAATCAC \\
\hline NatNT2 rev & CGATTCGTCGTCCGATTCGTC \\
\hline HIS-1 for & GAGCGCAAGGCGTTTATC \\
\hline Lys1_seq0 for & GTCATCCTCGAGAAACCACGATG \\
\hline Lys1-seq1 rev & GGCTCTAACCCAGACAGGAGCAG \\
\hline Arg4_seq0 for & GGTCAGCGAAGCACAGAACTC \\
\hline Arg4-seq1 rev & GGTTCCACCAGTAGCATCTCGTC \\
\hline Atg5_K1 for & GAAGTAGCATGCTCAGAAGTG \\
\hline Atg5_K3 rev & CAACGTAGGATTGACTCCAGTC \\
\hline Atg21 pro for & GAGCGTGAGCTGCAGAAAG \\
\hline atg21-seq6 rev & CCTTTGTTTAGACCTTGTCCTC \\
\hline Atg3 for & GATTACGTACACTTGACG \\
\hline Atg3 rev & GGAAGAAACTTGTTCCTGC \\
\hline Vph1_k1 for & GCCATGTGGTTCGCACTAAC \\
\hline Vph1_k1_rev & CTGAACACGGGGCAGTGC \\
\hline atg14_K1 for & GGGAAAGGACCAAATACAAAAGTG \\
\hline Atg14_k1 rev & ССTTTTCTTTCAACGGGGTG \\
\hline Vac8_K3 rev & CGAGCTTGGATTGTTGTTGCTCCCC \\
\hline
\end{tabular}




\begin{tabular}{ll}
\hline Vac8_K4 for & CTTTTCGTCACTGACGGGTCCCTGC \\
\hline Vac8_K5rev & CCCACCATCAGAGGACGAAGAGAG \\
\hline Myc_BirA_XbaI for & AGTCAATCTAGAATGGAACAGAAACTGATCTCTG \\
\hline BirA_XbaI rev & AGTCAATCTAGATTTTTCTGCACTACGCAGGG \\
\hline pUG35_Myc_BirA for & $\begin{array}{l}\text { CCCGGGCTGCAGGAATTCGATATCAAGCTTATCGATACCGTCGAC } \\
\text { GAACAGAAACTGATCTCTGAAG }\end{array}$ \\
\hline pUG35_BirA rev & $\begin{array}{l}\text { GGCGTGAATGTAAGCGTGACATAACTAATTACATGACTCGACCAG } \\
\text { CTCGAGCTATTTTTCTGCACTAC }\end{array}$ \\
\hline BamHI_Atg5 for & AGTCAAGGATCCATGAATGACATTAAACAATTAC \\
\hline Atg5_XhoI rev & AGTCAACTCGAGTTAGAGCTCAGAGGAAGCTTTATC \\
\hline SalI-Atg5 for & AGTCAAGTCGACATGAATGACATTAAACAATTACTTTGG \\
\hline Atg5-2xFLAG rev & TTGACTCTCGAGTTACTTATCATCATCATCCTTGTAATCCTTATCA \\
\hline SacI-PAtg18 for & TCATCATCCTTGTAATCGGATCCGAGCTCAGAGGAAGCTTTATCG \\
\hline EcoRI-Atg18ws rev & TTGACTGAATTCATCCATCAAGATGGAATACTGTGAC \\
\hline SacI-PVac8 for & AGTCAAGAGCTCGAGGACTCAAAACGAAAAGG \\
\hline Vac8_EcoRI rev & TTGACTGAATTCATGTAAAAATTGTAAAATCTGTTGAG \\
\hline
\end{tabular}

Table 3.6: Oligonucleotides for gene deletion and chromosomal tagging used in this study

\begin{tabular}{|c|c|}
\hline Name & Sequence \\
\hline Arg4_S1 & $\begin{array}{l}\text { CAATTGAAGAGCTCAAAAGCAGGTAACTATATAACAAGACTAAGGCA } \\
\text { AACATGCGTACGCTGCAGGTCGAC }\end{array}$ \\
\hline Arg4_S2 & $\begin{array}{l}\text { CCTAGAAGTACCAGACCTGATGAAATTCTTGCGCATAACGTCGCCATC } \\
\text { TGCTAATCGATGAATTCGAGCTCG }\end{array}$ \\
\hline Lys1_S1 & $\begin{array}{l}\text { GCATACCATAAGATAACAACGAAAACGCTTTATTTTTCACACAACCG } \\
\text { CAAAAATGCGTACGCTGCAGGTCGAC }\end{array}$ \\
\hline Lys1_S2 & $\begin{array}{l}\text { CTTGTAAATGTCAGCGTAACGATAATGTATATACTTTAAATGTAAAC } \\
\text { TAATCGATGAATTCGAGCTCG }\end{array}$ \\
\hline Atg21 KO_S1 & $\begin{array}{l}\text { CAAAAGACAATTCCACTCCTTTGGATTTGAAATAGACAGATAGAAAA } \\
\text { GGATATGCGTACGCTGCAGGTCGAC }\end{array}$ \\
\hline Atg21 KO_S2 & $\begin{array}{l}\text { CGTGAATACGTACAATATCTATTAAGATTATGAAAACTGCACATATG } \\
\text { CATTAATCGATGAATTCGAGCTCG }\end{array}$ \\
\hline Atg5::NAT for & $\begin{array}{l}\text { GTTCTTTTGGTTCTAGAAGAACGGAGATAGGAAACCTATGATGTAAG } \\
\text { TATGCGTACGCTGCAGGTCGAC }\end{array}$ \\
\hline Atg5::NAT rev & $\begin{array}{l}\text { CTGCGATATTTGAATGACACTTTTAAATGCGTATATAACAGCTCTTA } \\
\text { ATCGATGAATTCGAGCTCG }\end{array}$ \\
\hline Ape1_S3 & $\begin{array}{l}\text { GGATTTTTCAAGCACTGGAGATCAGTCTACGATGAATTCGGCGAGTT } \\
\text { GCGTACGCTGCAGGTCGAC }\end{array}$ \\
\hline Ape1_S2 & $\begin{array}{l}\text { GGATAAAGAAACAGAAATCAAAAGAAATAAAAAGAGTGTGGCAAAA } \\
\text { TCAATCGATGAATTCGAGCTCG }\end{array}$ \\
\hline Atg3 S1 & $\begin{array}{l}\text { GCTAGCTAGAAGTTAGGAACAAAGAAGTACAAAGGAGTAAATACAAT } \\
\text { TTTATTATCATGCGTACGCTGCAGGTCGAC }\end{array}$ \\
\hline Atg3 S2 & $\begin{array}{l}\text { GTTTATCCTGTTTTTTGACCACCTGGCTTGCAGCTAATAGTGAAAAA } \\
\text { ACACAAATTTTTAATCGATGAATTCGAGCTCG }\end{array}$ \\
\hline Vph1_S2 & $\begin{array}{l}\text { GAAGTACTTAAATGTTTCGCTTTTTTTAAAAGTCCTCAAAATTTAAT } \\
\text { CGATGAATTCGAGCTCG }\end{array}$ \\
\hline Vph1_S3 & $\begin{array}{l}\text { GACATGGAAGTCGCTGTTGCTAGTGCAAGCTCTTCCGCTTCAAGCCGT } \\
\text { ACGCTGCAGGTCGAC }\end{array}$ \\
\hline Atg14-3xGFP for & $\begin{array}{l}\text { CCTCCAACAGTCACAGGACCTCATGACCGATGGTACGTGGTAGGCCCG } \\
\text { CCAGCTGAAGCTTGG }\end{array}$ \\
\hline
\end{tabular}




\begin{tabular}{ll}
\hline Atg14-3xGFP rev & $\begin{array}{l}\text { CTACATGCAACTTTATACACACGGCAGGAAAAAAAGTGCGCACTCTA } \\
\text { CGTTTAAACTGGATGGCGGCG }\end{array}$ \\
\hline Vac8-3xGFP for & $\begin{array}{l}\text { GTTTGGAATTGTATAATATTACTCAACAGATTTTACAATTTTTACAT } \\
\text { CCGCCAGCTGAAGCTTGG }\end{array}$ \\
\hline \multirow{2}{*}{ Vac8-3xGFP rev } & $\begin{array}{l}\text { AAAATTTTGATAAAAATTATAATGCCTAGTCCCGCTTTTGAAGAAAA } \\
\text { TCACGTTTAAACTGGATGGCGGCG }\end{array}$ \\
\hline \multirow{2}{*}{ Vac8-NAT for } & $\begin{array}{l}\text { CTATAAGGGTGTTCTTTCTTCTGTACTATATATACATTTGCAACTAT } \\
\text { GCGTACGCTGCAGGTCGAC }\end{array}$ \\
\hline \multirow{2}{*}{ Vac8-NAT rev } & AAAATTTTGATAAAAATTATAATGCCTAGTCCCGCTTTTGAAGAAAA \\
\hline \multirow{2}{*}{ Vac8_S3 } & CCAATCGATGAATTCGAGCTCG \\
\hline
\end{tabular}

Table 3.7: Oligonucleotides for site-directed mutagenesis used in this study

The exchanged bases can be discriminated by lower case letters.

\begin{tabular}{ll}
\hline Name & Sequence \\
\hline Atg21R151E for & CCACATGAAATTGTTGATGTGGTCATGAATgaAAAAAGAATGTGC \\
\hline Atg21R151E rev & GCACATTCTTTTTtcATTCATGACCACATCAACAATTTCATGTGG \\
\hline Atg21K152E for & GGTCATGAATAGAgAAAGAATGTGCGTACTTCTTGAAAGTGACC \\
\hline Atg21K152E rev & GGTCACTTTCAAGAAGTACGCACATTCTTTcTCTATTCATGACC \\
\hline Atg21R151EK152E & GGTCATGAATgaAgAAAGAATGTGCGTACTTCTTGAAAGTGACC \\
for & \\
\hline Atg21R151EK152E & GGTCACTTTCAAGAAGTACGCACATTCTTTcTtcATTCATGACC \\
rev & \\
\hline Mut_Atg16_RR for & GAATACGGAAAGGTTGAATcgCagATTGATTAGTGGAACCATTG \\
\hline Mut_Atg16_RR rev & CAATGGTTCCACTAATCAATctGcgATTCAACCTTTCCGTATTC \\
\hline Atg16_D101R for & GAATACGGAAAGGTTGAATcgCGAATTGATTAGTGGAACCATTG \\
\hline Atg16_D101R rev & CAATGGTTCCACTAATCAATTCGcgATTCAACCTTTCCGTATTC \\
\hline Atg16_E102R for & GAATACGGAAAGGTTGAATGACagATTGATTAGTGGAACCATTG \\
\hline Atg16_E102R rev & CAATGGTTCCACTAATCAATctGTCATTCAACCTTTCCGTATTC \\
\hline Atg21_D9R for & GTATTACAATTCAATCAAcgTGCAACGTGCTGTGTGGTGGCCGCG \\
\hline Atg21_D9R rev & CGCGGCCACCACACAGCACGTTGCAcgTTGATTGAATTGTAATAC \\
\hline Atg21_D28R for & CGATTTTTAACTGCcgCCCCTTTGGTAAATGTTTTGAAATTGAC \\
\hline Atg21_D28R rev & GTCAATTTCAAAACATTTACCAAAGGGGcgGCAGTTAAAAATCG \\
\hline Atg21_K130E for & GCTGAAGATTGTCAATACAgAGAGAAAATGTACTATTTGTG \\
\hline Atg21_K130E rev & CACAAATAGTACATTTTCTCTcTGTATTGACAATCTTCAGC \\
\hline Atg21_E137R for & GTACTATTTGTcgAATAGTTTTCCCACATGAAATTGTTGATGTGG \\
\hline Atg21_E137R rev & CCACATCAACAATTTCATGTGGGAAAACTATTCgACAAATAGTAC \\
\hline Atg21_D167R for & GAAAGTGACCAGATATTCATTTATcgTATATCTTGTATGAAACCC \\
\hline Atg21_D167R rev & GGGTTTCATACAAGATATAcgATAAATGAATATCTGGTCACTTTC \\
\hline Atg21_K172E for & GATATATCTTGTATGgAACCCTTAGAAACTATCGATCTTTGGG \\
\hline Atg21_K172E rev & CCCAAAGATCGATAGTTTCTAAGGGTTCCATACAAGATATATC \\
\hline Atg21_ST_AA for & GGGGAGTTTGTCATTGAAATGTTGTTTgCAgCTAGTCTTATTGC \\
\hline Atg21_ST_AA rev & GCAATAAGACTAGcTGcAAACAACATTTCAATGACAAACTCCCC \\
\hline Atg16_K78E for & CAAAAAGAACTGAAAAGTgAGGAGCAAGAAATACGAAGATTG \\
\hline Atg16_K78E rev & CAATCTTCGTATTTCTTGCTCCTcACTTTTCAGTTCTTTTTG \\
\hline Atg16_E81R for & GAAAAGTAAGGAGCAAcgAATACGAAGATTGAAAGAAGTTATTGC \\
\hline Atg16_E81R rev & G \\
\hline Atg16_K94E for & GTTATTGCGTTGAAAAATgAGAATACGGAAAGGTTGAATGACG \\
\hline Atg16_K94E rev & CGTCATTCAACCTTTCCGTATTCTcATTTTTCAACGCAATAAC \\
\hline
\end{tabular}




\begin{tabular}{ll}
\hline atg21 mut for & GGTCACTGTTTAAAGAATTTAcaacGGGTACCAGATTGTGC \\
\hline $\begin{array}{l}\text { atg21 mut rev } \\
\text { Atg3_W270A_L273A }\end{array}$ & GCACAATCTGGTACCCgttgTAAATCTTTAAACAGTGACC \\
\hline $\begin{array}{l}\text { Atg3_W270A_L273A } \\
\text { rev }\end{array}$ & GGTGTCGGAGACgcGGAGGATgcACAAGACGATATTGATGATTCG \\
\hline Atg3_C234A for & $\begin{array}{l}\text { CAGTGTTATCTGTTTCCATTCATCCAgcTAAGCATGCTAATGTAAT } \\
\text { G }\end{array}$ \\
\hline Atg3_C234A rev & $\begin{array}{l}\text { CATTACATTAGCATGCTTAgcTGGATGAATGGAAACAGATAACAC } \\
\text { TG }\end{array}$ \\
\hline
\end{tabular}

\subsubsection{Antibodies}

The antibodies in Table 3.8 were diluted in TBST containing 1\% (w/v) skim milk powder if not stated differently.

Table 3.8: Antibodies used in this study

\begin{tabular}{|c|c|c|}
\hline Antibody & Dilution & Source \\
\hline $5 F 8 \alpha-\operatorname{Red}($ rat mAb) & $1: 1000$ & Chromotek (Munich) \\
\hline 6G6 $\alpha$-Red (mouse mAb) & $1: 1000$ & Chromotek (Munich) \\
\hline Anti-GFP (from mouse IgG1א) & $1: 1000$ & Roche (Mannheim) \\
\hline $\begin{array}{l}\text { Goat IgG anti-Mouse IgG } \\
(\mathrm{H}+\mathrm{L})-\mathrm{HRPO}\end{array}$ & $1: 10000$ & Dianova (Hamburg) \\
\hline $\begin{array}{l}\text { Goat IgG anti-Rat IgG }(\mathrm{H}+\mathrm{L})- \\
\text { HRPO }\end{array}$ & $1: 10000$ & Dianova (Hamburg) \\
\hline $\begin{array}{l}\text { Goat anti-Rabbit IgG (H+L) } \\
\text { Cross-Adsorbed Secondary } \\
\text { Antibody, HRP }\end{array}$ & $1: 5000$ & $\begin{array}{l}\text { Thermo Fisher Scientific } \\
\text { (Bremen) }\end{array}$ \\
\hline $\begin{array}{l}\text { HA-probe Antibody (F-7) } \\
\text { (mouse monoclonal } \operatorname{IgG}_{2 \mathrm{a}} \text { ) }\end{array}$ & $1: 10000$ & $\begin{array}{l}\text { Santa Cruz Biotechnology } \\
\text { (Dallas, USA) }\end{array}$ \\
\hline $\begin{array}{l}\text { Monoclonal ANTI-FLAG }{ }^{\circledR} \text { M2 } \\
\text { antibody produced in mouse }\end{array}$ & 1:500 (in TBST) & Sigma-Aldrich (Munich) \\
\hline Mouse IgG anti-Myc & 1:500 (in TBST) & $\begin{array}{l}\text { Gift from the group of Prof. Dr. } \\
\text { P. Rehling (Dept. Cellular } \\
\text { Biochemistry; University } \\
\text { Göttingen) }\end{array}$ \\
\hline rabbit IgG anti-Ape1 & $1: 5000$ & Eurogentec (Seraing, Belgium) \\
\hline Strep-Tactin HRP conjugate & 1:50000 (in TBST)* & iba (Göttingen) \\
\hline
\end{tabular}

\subsubsection{Commercially available kits and master mixes}

Kit systems and master mixes listed in Table 3.9 were used according to the manufacturer's recommendations. 
Table 3.9: Commercially available kits and master mixes used in this study

\begin{tabular}{|c|c|}
\hline Name of the kit & Source \\
\hline DreamTaq Green PCR Master Mix (2x) & Thermo Fisher Scientific (Bremen) \\
\hline $\begin{array}{l}\text { Amersham }{ }^{\mathrm{TM}} \text { ECL }^{\mathrm{TM}} \text { Western-Blotting Detection } \\
\text { Reagents }\end{array}$ & GE Healthcare (Solingen) \\
\hline NucleoSpin ${ }^{\circledR}$ Microbial DNA & Macherey-Nagel (Düren) \\
\hline Pierce $^{\mathrm{TM}}$ ECL Plus Western-Blotting Substrate & Thermo Fisher Scientific (Bremen) \\
\hline QIAquick Gel Extraction Kit & Qiagen (Hilden) \\
\hline QIAquick PCR Purification Kit & Qiagen (Hilden) \\
\hline QuickChange II Site-Directed Mutagenesis Kit & Agilent (Santa Clara, USA) \\
\hline $\begin{array}{l}\text { QuickChange Lightning Site-Directed Mutagenesis } \\
\text { Kit }\end{array}$ & Agilent (Santa Clara, USA) \\
\hline Wizard ${ }^{\circledR}$ Plus SV Miniprep System & Promega (Mannheim) \\
\hline
\end{tabular}

\subsubsection{Chemicals and Consumables}

Standard chemicals were used in analytical grade quality and obtained from the following companies: AppliChem (Darmstadt), Roth (Karlsruhe), Serva (Heidelberg), Sigma-Aldrich (Munich) and Merck (Darmstadt). Special chemicals are listed in Table 3.10 and enzymes in Table 3.11.

Table 3.10: Chemicals, supplements and protein purification systems used in this study

\begin{tabular}{|c|c|}
\hline Name of product & Source \\
\hline${ }^{13} \mathrm{C}_{6}$-L-Arginine $\mathrm{HCl}($ Arg-6:HCl) & Silantes (Munich) \\
\hline${ }^{13} \mathrm{C}_{6},{ }^{15} \mathrm{~N}_{4}$-L-Arginine $\mathrm{HCl}$ (Arg-10:HCl) & Silantes (Munich) \\
\hline${ }^{13} \mathrm{C}_{6},{ }^{15} \mathrm{~N}_{2}$-L-Lysine $\mathrm{HCl}$ (Lys-8:HCl) & Silantes (Munich) \\
\hline 4,4,5,5-D -L-Lysine 2HCl (Lys-4D:2HCl) & Silantes (Munich) \\
\hline 5-Fluoroorotic acid & Thermo Fisher Scientific (Bremen) \\
\hline Ampicillin sodium salt & Roth (Karlsruhe) \\
\hline Agarose NEEO Ultra-Qualität & Roth (Karlsruhe) \\
\hline Bacto $^{\mathrm{TM}}$ Agar & Becton Dickinson (Heidelberg) \\
\hline Bacto $^{\mathrm{TM}}$ Pepton & Becton Dickinson (Heidelberg) \\
\hline Bacto $^{\mathrm{TM}}$ Trypton & Becton Dickinson (Heidelberg) \\
\hline Bacto $^{\mathrm{TM}}$ Yeast Extract & Becton Dickinson (Heidelberg) \\
\hline Biotin & Sigma-Aldrich (Munich) \\
\hline $\begin{array}{l}\text { Buffer W (10x) (Strep-Tactin }{ }^{\circledR} / \text { Strep-Tactin }{ }^{\circledR X T} \\
\text { Wash Buffer) }\end{array}$ & iba (Göttingen) \\
\hline Coomassie ${ }^{\circledR}$ Brilliant Blue G 250 & Serva (Heidelberg) \\
\hline cOmplete ${ }^{\mathrm{TM}}, \mathrm{EDTA}-$ free & Roche (Mannheim) \\
\hline DeltaVision Immersion Oil (N=1.520) & GE Healthcare (Solingen) \\
\hline Deoxyadenosine triphosphate (dATP) & New England Biolabs (Frankfurt) \\
\hline Deoxycytidine triphosphate (dCTP) & New England Biolabs (Frankfurt) \\
\hline Deoxyguanosine triphosphate (dGTP) & New England Biolabs (Frankfurt) \\
\hline Deoxythymidine triphosphate (dTTP) & New England Biolabs (Frankfurt) \\
\hline Difco $^{\mathrm{TM}}$ Yeast nitrogen base $\mathrm{w} / \mathrm{o}$ amino acids & Becton Dickinson (Heidelberg) \\
\hline $\begin{array}{l}\text { Difco }{ }^{\mathrm{TM}} \text { Yeast nitrogen base } \mathrm{w} / \mathrm{o} \text { amino acids and } \\
\text { ammonim sulfate }\end{array}$ & Becton Dickinson (Heidelberg) \\
\hline
\end{tabular}




\begin{tabular}{|c|c|}
\hline $\begin{array}{l}\text { Drop-out Mix Synthetic, minus Ade, Arg, His, Leu, } \\
\text { Lys, Met, Trp, Ura w/o Yeast Nitrogen Base }\end{array}$ & USBiological (Salem, USA) \\
\hline Ethidium bromide solution $(0.025 \%)$ & Roth (Karlsruhe) \\
\hline Frema Reform Instant Skim Milk Powder & Granovita (Heimertingen) \\
\hline Gel Loading Dye, Purple (6X) & New England Biolabs (Frankfurt) \\
\hline GFP-Trap ${ }^{\circledR}$ & Chromotek (Munich) \\
\hline $\begin{array}{l}\text { Gravity flow Strep-Tactin }{ }^{\circledR} \text { Sepharose }{ }^{\circledR} \text { Column } \\
(0.2 \mathrm{ml})\end{array}$ & iba (Göttingen) \\
\hline $\begin{array}{l}\text { Gravity flow Strep-Tactin }{ }^{\circledR} \text { Sepharose }^{\circledR} \text { Column } \\
(1 \mathrm{ml})\end{array}$ & iba (Göttingen) \\
\hline Herring Sperm DNA & Promega (Mannheim) \\
\hline Hygromycin B solution & Roth (Karlsruhe) \\
\hline Invitrogen ${ }^{\mathrm{TM}}$ Molecular Probes ${ }^{\mathrm{TM}} \mathrm{FM}$ 4-64 Dye & Thermo Fisher Scientific (Bremen) \\
\hline Invitrogen ${ }^{\mathrm{TM}}$ Molecular Probes ${ }^{\mathrm{TM}}$ Hoechst 33342 & Thermo Fisher Scientific (Bremen) \\
\hline $\begin{array}{l}\text { Nourseothricin-dihydrogen sulfate/clonNAT } \\
\text { powder }\end{array}$ & Werner BioAgents (Jena) \\
\hline $\begin{array}{l}\text { Precision Plus Protein }{ }^{\mathrm{TM}} \text { All Blue Prestained } \\
\text { Protein Standard }\end{array}$ & Bio-Rad (Munich) \\
\hline Rapamycin & LC Laboratories (Woburn, USA) \\
\hline Strep-Tactin ${ }^{\circledR}$ Spin Column & iba (Göttingen) \\
\hline TriDye $^{\mathrm{TM}} 1$ kb DNA Ladder & New England Biolabs (Frankfurt) \\
\hline
\end{tabular}

Table 3.11: Enzymes used in this study

\begin{tabular}{ll}
\hline Enzyme & Source \\
\hline Alkaline Phosphatase, Calf Intestinal (CIP) & New England Biolabs (Frankfurt) \\
\hline FideliTaq ${ }^{\text {TM }}$ DNA polymerase & Affymetrix (Santa Clara, USA) \\
\hline KOD Hot Start DNA Polymerase & Merck (Darmstadt) \\
\hline $\begin{array}{l}\text { DNA Polymerase I, Large (Klenow) } \\
\text { Fragment }\end{array}$ & New England Biolabs (Frankfurt) \\
\hline Restriction enzymes & New England Biolabs (Frankfurt) \\
\hline Taq DNA polymerase & New England Biolabs (Frankfurt) \\
\hline T4-DNA-Ligase & New England Biolabs (Frankfurt) \\
\hline Vent ${ }^{\circledR}$ DNA polymerase & New England Biolabs (Frankfurt) \\
\hline Zymolyase 100T & Seikagaku Biobusiness (Tokyo, Japan) \\
\hline
\end{tabular}

\subsubsection{Equipment}

Table 3.12: Equipment used in this study

\begin{tabular}{ll}
\hline Name of product & Source \\
\hline $\begin{array}{l}\text { Agarose gel equipment Bio-Rad Mini-SUB } \\
\text { Cell GT }\end{array}$ & Bio-Rad (Munich) \\
\hline Amersham ${ }^{\mathrm{TM}}$ Hybond ${ }^{\mathrm{TM}}$ P0.45 PVDF & GE Healthcare (Solingen) \\
\hline Autoclave Systec DE-200 & Systec (Linden) \\
\hline BioPhotometer 6131 Spectrophotometer & Eppendorf (Hamburg) \\
\hline Blot Shaker GFL ${ }^{\circledR} 3019$ & GFL (Burgwedel) \\
\hline Blotting Paper (MN218B $58 \times 60 \mathrm{~cm}$ ) & Macherey-Nagel (Düren) \\
\hline Clean Bench & $\begin{array}{l}\text { BDK Luft- und Reinraumtechnik } \\
\text { (Sonnenbühl-Genkingen) }\end{array}$ \\
\hline
\end{tabular}




\begin{tabular}{|c|c|}
\hline Centrifuge 5415D & Eppendorf (Hamburg) \\
\hline Centrifuge 5415R & Eppendorf (Hamburg) \\
\hline Centrifuge $5417 \mathrm{C}$ & Eppendorf (Hamburg) \\
\hline Centrifuge 5804 & Eppendorf (Hamburg) \\
\hline Centrifuge 5804R & Eppendorf (Hamburg) \\
\hline Consort Power Supplies E831 & Consort NV (Turnhout, Belgium) \\
\hline $\begin{array}{l}\text { Corning }^{\mathrm{TM}} \text { Stripettor } \\
\text { Controller }\end{array}$ & Corning Incorporated (Corning, USA) \\
\hline Cuvettes no. $67.742(10 \times 4$ x 45 mm $)$ & Sarstedt (Nümbrecht) \\
\hline Dispensette ${ }^{\circledR} 25 \mathrm{ml}$ & Brand (Wertheim) \\
\hline Disruptor Genie ${ }^{\circledR}$ Vortex-Shaker & Scientific Industries (Bohemia, USA) \\
\hline Freezer $\left(-20^{\circ} \mathrm{C}\right)$ & Liebherr (Biberach) \\
\hline Freezer $\left(-80^{\circ}\right)$ & Heareus (Hanau) \\
\hline Gasprofi 1 SCS micro & WLD-Tec (Göttingen) \\
\hline Glass beads, acid washed & Sigma-Aldrich (Munich) \\
\hline IKA ${ }^{\circledR}$ VIBRAX VXR basic & IKA (Staufen) \\
\hline Innova 4200 Incubator-Shaker $\left(37^{\circ} \mathrm{C}\right)$ & New Brunswick Scientific (Nürtingen) \\
\hline JULABO MA-4 Heating Circulator & JULABO Labortechnik (Seelbach) \\
\hline Lab balance Sartorius Handy H51-D & Sartorius (Göttingen) \\
\hline Lab balance Sartorius Universal U4100 & Sartorius (Göttingen) \\
\hline Lab pH meter inoLab® pH 7110 & Xylem Analytics (Weilheim) \\
\hline Lab-shaker LS-X & Kühner Shaker (Herzogenrath) \\
\hline Lab-shaker SBM/SS-X (Rack-Shaker) & Kühner Shaker (Herzogenrath) \\
\hline LAS 3000 Intelligent Dark Box & Fuji Photo Film (Düsseldorf) \\
\hline Magnetic stirrer MR 2002 & Heidolph (Kelheim) \\
\hline Magnetic stirrer MR 3001 & Heidolph (Kelheim) \\
\hline $\begin{array}{l}\text { Menzel }^{\mathrm{TM}} \text { Microscope Coverslips } \\
(24 \times 24 \mathrm{~mm})\end{array}$ & Thermo Fisher Scientific (Bremen) \\
\hline 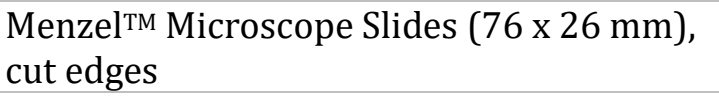 & Thermo Fisher Scientific (Bremen) \\
\hline Microscope DeltaVision $^{\circledR}$, Olympus IX71 & Applied Precision (Issaquah, USA) \\
\hline Microwave R-939 & Sharp Electronics (Hamburg) \\
\hline Minisart filters (pore size $0.2 \mu \mathrm{m}$ ) & Sartorius (Göttingen) \\
\hline $\begin{array}{l}\text { Nalgene }^{\circledR} \text { Rapid-Flow } 75 \text { mm Bottle Top } \\
\text { Filter, } 500 \mathrm{ml}\end{array}$ & Thermo Fisher Scientific (Bremen) \\
\hline NanoVue ${ }^{\mathrm{TM}}$ UV/Visible Spectrophotometer & GE Healthcare (Solingen) \\
\hline Nunc $^{\mathrm{TM}}$ OmniTray ${ }^{\mathrm{TM}}$, single well w/lid & Nalge Nunc International (Rochester, USA) \\
\hline PCR Mastercycler gradient & Eppendorf (Hamburg) \\
\hline pH Electrode SenTix ${ }^{\circledR} 41$ PLUS & Xylem Analytics (Weilheim) \\
\hline Pipettes research / research plus & Eppendorf (Hamburg) \\
\hline Pipette controllers accu-jet ${ }^{\circledR}$ pro & Brand (Wertheim) \\
\hline Potter S Homogenizer cylinder $2 \mathrm{ml}$ & Sartorius (Göttingen) \\
\hline Potter S Plunger made of PTFE $2 \mathrm{ml}$ & Sartorius (Göttingen) \\
\hline PowerPac ${ }^{\mathrm{TM}}$ Basic Power Supply & Bio-Rad (Munich) \\
\hline PowerPac ${ }^{\mathrm{TM}}$ HC Power Supply & Bio-Rad (Munich) \\
\hline Protein LoBind Tubes $1.5 \mathrm{ml}$ & Eppendorf (Hamburg) \\
\hline Refrigerator $\left(4^{\circ} \mathrm{C}\right)$ & Liebherr (Biberach) \\
\hline Roto Shake Genie ${ }^{\circledR}$ & Scientific Industries (Bohemia, USA) \\
\hline $\begin{array}{l}\text { SDS-PAGE equipment Bio-Rad Mini- } \\
\text { PROTEAN }{ }^{\circledR} \text { cell }\end{array}$ & Bio-Rad (Munich) \\
\hline Shaking Water Bath SWB25 & Thermo Haake (Karlsruhe) \\
\hline Sprout ${ }^{\circledR}$ Mini Centrifuge & Heathrow Scientific (Vernon Hills, USA) \\
\hline
\end{tabular}




\begin{tabular}{ll}
\hline Thermomixer comfort & Eppendorf (Hamburg) \\
\hline Transilluminator TI1 & Biometra (Göttingen) \\
\hline Transilluminator UVsolo & Biometra (Göttingen) \\
\hline Diaphragm vaccum pump & Vacuubrand (Wertheim) \\
\hline Vortex Genie $2^{\circledR}$ & Scientific Industries (Bohemia, USA) \\
\hline Semi-Dry Western-Blot Chambers & UMG (Göttingen) \\
\hline
\end{tabular}

\subsubsection{Media}

All media used for the cultivation of yeast or bacteria are listed in Table 3.13 or Table 3.16. They were prepared with deionized water $\left(\mathrm{ddH}_{2} \mathrm{O}\right)$ and the respective $\mathrm{pH}$ was adjusted using $\mathrm{NaOH}$ or $\mathrm{HCl}$. For sterilization the media were autoclaved at $121^{\circ} \mathrm{C}$ for $20 \mathrm{~min}$. Solid media plates were prepared by adding $2 \%$ of preheated sterile agar to the medium. To adjust the required selection conditions, a variety of supplements (Table 3.15) were added to the media.

Table 3.13: Yeast media used in this study

Percent values of this table indicate weight per volume $(\mathrm{w} / \mathrm{v})$.

\begin{tabular}{|c|c|c|c|}
\hline Name & Compos & tion & Reference \\
\hline & $1 \%$ & Bacto $^{\mathrm{TM}}$ Yeast extract & \\
\hline YPD medium, pH 5.5 & $2 \%$ & Bacto $^{\mathrm{TM}}$ Peptone & (sherman, \\
\hline & $2 \%$ & D-Glucose & \\
\hline & $0.67 \%$ & $\begin{array}{l}\text { Yeast Nitrogen Base w/o amino } \\
\text { acids }\end{array}$ & \\
\hline CM medium, pH 5.6 & $2 \%$ & D-Glucose & 1994) \\
\hline & $0.2 \%$ & Dropout-mix (see Table 3.14) & \\
\hline & $0.67 \%$ & $\begin{array}{l}\text { Yeast Nitrogen Base w/o amino } \\
\text { acids }\end{array}$ & \\
\hline $\mathrm{CM} \mathrm{w} / \mathrm{o}$ methionine & $2 \%$ & D-Glucose & (Kaiser et al., \\
\hline & $0.2 \%$ & $\begin{array}{l}\text { Dropout-mix w/o L-methionine (see } \\
\text { Table } 3.14 \text { ) }\end{array}$ & \\
\hline CM medium for & & $\begin{array}{l}\text { Drop-out Mix Synthetic, minus Ade, } \\
\text { Arg, His, Leu, Lys, Met, Trp, Ura w/o } \\
\text { Yeast Nitrogen Base }\end{array}$ & USBiological \\
\hline $\begin{array}{l}\text { SILAC experiments, } \\
\text { pH } 5.6\end{array}$ & $6.7 \mathrm{~g} / \mathrm{l}$ & $\begin{array}{l}\text { Yeast Nitrogen Base w/o amino } \\
\text { acids }\end{array}$ & (Salem, USA) \\
\hline & $2 \%$ & D-Glucose & \\
\hline SD-N medium & $0.67 \%$ & $\begin{array}{l}\text { Yeast Nitrogen Base } w / o \text { amino } \\
\text { acids and w/o ammonium sulfate }\end{array}$ & (Takeshige et \\
\hline & $2 \%$ & D-Glucose & \\
\hline MV medium & $0.67 \%$ & $\begin{array}{l}\text { Yeast Nitrogen Base w/o amino } \\
\text { acids }\end{array}$ & (Sherman, \\
\hline & $2 \%$ & D-Glucose & \\
\hline
\end{tabular}


Table 3.14: Composition of the drop-out mix that was used to prepare the CM medium according to Kaiser et al. (1994)

For the drop-out mix 2 g of each component were weighed out (except for the p-aminobenzoic acid where $0.2 \mathrm{~g}$ were used), grounded and thoroughly mixed. The drop-out mix lasts for $17 \mathrm{l}$ medium. For the drop-out mix w/o methionine the L-methionine was excluded from the mixture.

\begin{tabular}{ll} 
Compound & Concentration in medium \\
\hline L-alanine & $0.0117 \%$ \\
\hline L-arginine & $0.0117 \%$ \\
\hline L-asparagine & $0.0117 \%$ \\
\hline L-aspartic acid & $0.0117 \%$ \\
\hline L-cysteine & $0.0117 \%$ \\
\hline L-glutamine & $0.0117 \%$ \\
\hline L-glutamic acid & $0.0117 \%$ \\
\hline L-glycine & $0.0117 \%$ \\
\hline L-isoleucine & $0.0117 \%$ \\
\hline L-methionine & $0.0117 \%$ \\
\hline L-phenylalanine & $0.0117 \%$ \\
\hline L-proline & $0.0117 \%$ \\
\hline L-serine & $0.0117 \%$ \\
\hline L-tyreonine & $0.0117 \%$ \\
\hline L-valine & $0.0117 \%$ \\
\hline myo-inositol & $0.0117 \%$ \\
\hline p-aminobenzoic acid & $0.0117 \%$ \\
\hline
\end{tabular}

Table 3.15: Supplements for yeast media used in this study

Stock solutions were prepared in $\mathrm{ddH}_{2} \mathrm{O}$ if not stated differently and sterilized by autoclaving or filtration.

\begin{tabular}{lll}
\hline Supplement & Concentration in medium & stock solution \\
\hline${ }^{13} \mathrm{C}_{6}$-L-Arginine & $50 \mathrm{mg} / \mathrm{L}$ & $10 \mathrm{mg} / \mathrm{ml}$ \\
\hline${ }^{13} \mathrm{C}_{6}{ }^{15}{ }^{15}$-L-Arginine & $50 \mathrm{mg} / \mathrm{L}$ & $10 \mathrm{mg} / \mathrm{ml}$ \\
\hline${ }^{13} \mathrm{C}_{6}{ }^{15} \mathrm{~N}_{2}$-L-Lysine & $50 \mathrm{mg} / \mathrm{L}$ & $10 \mathrm{mg} / \mathrm{ml}$ \\
\hline $4,4,5,5-\mathrm{D}_{4}$-L-Lysine & $50 \mathrm{mg} / \mathrm{L}$ & $10 \mathrm{mg} / \mathrm{ml}$ \\
\hline 5 -FOA & $1 \mathrm{mg} / \mathrm{ml}$ & - \\
\hline Adenine & $300 \mu \mathrm{M}$ & $30 \mathrm{mM}$ \\
\hline Biotin & $10 \mu \mathrm{M}$ & $10 \mathrm{mM}$ \\
\hline ClonNat & $100 \mu \mathrm{g} / \mathrm{ml}$ & $100 \mathrm{mg} / \mathrm{ml}$ \\
\hline CuSO ${ }_{4}$ & $100 \mu \mathrm{M}$ & $100 \mathrm{mM}$ \\
\hline D-glucose & $2 \%(\mathrm{w} / \mathrm{v})$ & $40 \%(\mathrm{w} / \mathrm{v})$ \\
\hline FM4-64 & $20 \mu \mathrm{g} / \mathrm{ml}$ & $10 \mathrm{mg} / \mathrm{ml}$ \\
\hline Hoechst & $12.5 \mu \mathrm{g} / \mathrm{ml}$ & $10 \mathrm{mg} / \mathrm{ml}$ \\
\hline Hygromycin B solution & $0.3 \mathrm{mg} / \mathrm{ml}$ & $50 \mathrm{mg} / \mathrm{ml}$ \\
\hline L-arginine & $50 \mathrm{mg} / \mathrm{L}$ & $10 \mathrm{mg} / \mathrm{ml}$ \\
\hline L-histidine & $300 \mu \mathrm{M}$ & $60 \mathrm{mM}$ \\
\hline L-leucine & $1.67 \mathrm{mM}$ & $100 \mathrm{mM}$ \\
\hline L-lysine & $50 \mathrm{mg} / \mathrm{L}$ & $10 \mathrm{mg} / \mathrm{ml}$ \\
\hline L-methionine & $300 \mu \mathrm{M}$ & $300 \mathrm{mM}$ \\
\hline L-tryptophan & $400 \mu \mathrm{M}$ & $40 \mathrm{mM}$ \\
\hline Rapamycin & $400 \mathrm{ng} / \mathrm{ml}$ & $1 \mathrm{mg} / \mathrm{ml} \mathrm{in} \mathrm{DMSO}$ \\
\hline Uracil & $200 \mu \mathrm{M}$ & $20 \mathrm{mM}$ \\
\hline
\end{tabular}


Table 3.16: E. coli media used in this study

Percent values of this table indicate weight per volume $(\mathrm{w} / \mathrm{v})$.

\begin{tabular}{llll}
\hline Name & Composition & Reference \\
\hline & $1 \%$ & Bacto $^{\text {TM }}$ Trypton & \\
LB medium, 7.5 & $0.5 \%$ & Bacto $^{\text {TM }}$ Yeast extract & (Bertani, 1951) \\
& $0.5 \%$ & Sodium chloride & \\
& $0.75 \mu \mathrm{g} / \mathrm{ml}$ & Ampicillin & \\
& $2 \%$ & Bacto $^{\text {TM }}$ Trypton & \\
SOC medium & $0.5 \%$ & Bacto $^{\text {TM }}$ Yeast extract & \\
pH 7.5 & $0.4 \%$ & D-Glucose & \\
& $10 \mathrm{mM}$ & Sodium chloride & \\
& $10 \mathrm{mM}$ & Magnesium sulfate & \\
& $10 \mathrm{mM}$ & Magnesium chloride & \\
& $2.5 \mathrm{mM}$ & Potassium chloride & \\
\hline
\end{tabular}

\subsubsection{Standard buffers}

Table 3.17: Composition of regularly used buffers in this study

\begin{tabular}{|c|c|c|c|}
\hline Buffer & Composition & & Usage \\
\hline \multirow{4}{*}{ PEG in LiTE buffer } & $100 \mathrm{mM}$ & Lithium acetate & \multirow{8}{*}{$\begin{array}{l}\text { Yeast } \\
\text { transformation }\end{array}$} \\
\hline & $10 \mathrm{mM}$ & Tris/ acetic acid pH 8.0 & \\
\hline & $1 \mathrm{mM}$ & EDTA & \\
\hline & $40 \%(\mathrm{w} / \mathrm{v})$ & PEG 3350 & \\
\hline \multirow{4}{*}{ LiOAc-Sorb buffer } & $100 \mathrm{mM}$ & Lithium acetate & \\
\hline & $10 \mathrm{mM}$ & Tris/ acetic acid pH 8.0 & \\
\hline & $1 \mathrm{mM}$ & EDTA & \\
\hline & $1 \mathrm{M}$ & D-Sorbitol & \\
\hline \multirow{3}{*}{ SDS running buffer } & $25 \mathrm{mM}$ & Tris & \multirow{10}{*}{$\begin{array}{l}\text { SDS-Polyacrylamide } \\
\text { gel electrophoresis }\end{array}$} \\
\hline & $200 \mathrm{mM}$ & Glycine & \\
\hline & $0.1 \%$ & SDS & \\
\hline \multirow{5}{*}{ 6x Laemmli buffer } & $350 \mathrm{mM}$ & Tris/ HCl pH 6.8 & \\
\hline & $36 \%(\mathrm{w} / \mathrm{v})$ & Glycerol & \\
\hline & $10.28 \%(\mathrm{w} / \mathrm{v})$ & SDS & \\
\hline & $0.012 \%(\mathrm{w} / \mathrm{v})$ & Bromophenol blue & \\
\hline & $5 \%(\mathrm{v} / \mathrm{v})$ & $\begin{array}{l}\beta \text {-mercaptoethanol } \\
\text { (freshly added) }\end{array}$ & \\
\hline 4x Laemmli buffer & $66.7 \%(\mathrm{v} / \mathrm{v})$ & 6x Laemmli buffer & \\
\hline 2x Laemmli buffer & $33.3 \%(\mathrm{v} / \mathrm{v})$ & 6x Laemmli buffer & \\
\hline \multirow{3}{*}{ Blotting buffer } & $192 \mathrm{mM}$ & Glycine & \multirow{6}{*}{$\begin{array}{l}\text { Western-Blot } \\
\text { Analysis }\end{array}$} \\
\hline & $25 \mathrm{mM}$ & Tris & \\
\hline & $20 \%$ & Ethanol & \\
\hline \multirow{3}{*}{ TBST buffer } & $20 \mathrm{mM}$ & Tris/ HCl pH 7.6 & \\
\hline & 137 mM & Sodium chloride & \\
\hline & $0.1 \%(\mathrm{w} / \mathrm{v})$ & Tween20 & \\
\hline TAE buffer & $\begin{array}{l}40 \mathrm{mM} \\
2 \mathrm{mM} \\
0.114 \%(\mathrm{v} / \mathrm{v})\end{array}$ & $\begin{array}{l}\text { Tris/ acetic acid pH } 8.1 \\
\text { EDTA } \\
\text { acetic acid }\end{array}$ & $\begin{array}{l}\text { Agarose gel } \\
\text { electrophoresis }\end{array}$ \\
\hline
\end{tabular}




\begin{tabular}{llll}
\hline & $1 \mathrm{mg} / \mathrm{ml}$ & Antipain & \\
Protease Inhibitor & $1 \mathrm{mg} / \mathrm{ml}$ & Aprotinin & Cell lysis \\
Mix & $1 \mathrm{mg} / \mathrm{ml}$ & Pepstatin & of $1: 1000$ ) \\
& $1 \mathrm{mg} / \mathrm{ml}$ & Leupeptin & \\
& $1 \mathrm{mg} / \mathrm{ml}$ & Chymostatin & \\
\hline & $140 \mathrm{mM}$ & Sodium chloride & \\
1 x PBS pH 7.4 & $2.7 \mathrm{mM}$ & Potassium chloride $^{\text {GFP-TRAP }}$ & \\
& $10 \mathrm{mM}$ & $\mathrm{Na}_{2} \mathrm{HPO}_{4}$ & \\
& $1.8 \mathrm{mM}$ & $\mathrm{KH}_{2} \mathrm{PO}_{4}$ & \\
\hline
\end{tabular}

\subsection{Cultivation conditions}

\subsubsection{Cultivation of S. cerevisiae}

\subsubsection{Liquid cultivation}

Liquid yeast cultures were regularly grown at $30^{\circ} \mathrm{C}$ and shaking with $220 \mathrm{rpm}$. Yeast precultures were inoculated with a sterile toothpick from an agar plate and incubated for up to $24 \mathrm{~h}$. The precultures were used to inoculate the main cultures. To achieve the desired cell density of the main culture, a defined dilution of the preculture was used depending on the growth ability of the respective strain. The main cultures were also grown over night (15-17 h).

YPD medium was used as nutrient rich medium. The CM medium is a synthetic medium and was used to select for genetic markers. It was supplemented with different amino acids depending on the selection conditions. For overexpression experiments using the MET25 promoter the CM w/o L-methionine medium was used where L-methionine was excluded from the drop-out mix (Table 3.14). If a lower expression level from the MET25 promoter was desired $0.3 \mathrm{mM}$ L-methionine was supplemented into the medium otherwise normal CM medium with $0.78 \mathrm{mM}$ L-methionine was used. In case of overexpression from the CUP1 promoter either normal CM medium was used or it was supplemented with $100 \mu \mathrm{M}$ copper sulfate depending on the desired expression level.

\subsubsection{Short- and long-term storage}

Over a period of 4-6 weeks yeast strains were stored on agar plates at $4^{\circ} \mathrm{C}$. For the long-term storage cryo-stocks were prepared by adding $30 \%(\mathrm{w} / \mathrm{v})$ sterile glycerol 
to a yeast liquid culture (grown over night) in a 1:1 ratio. The cryo-stocks were stored at $-80^{\circ} \mathrm{C}$.

\subsubsection{Determination of cell density}

The cell density of liquid cultures was determined by photometry. Typically, the cultures were diluted by a factor of 10 in sterile medium and the optical density at a wavelength of $600 \mathrm{~nm}\left(\mathrm{OD}_{600}\right)$ was measured in a photometer. As a reference, the sterile medium was used. For cell densities below $1 \mathrm{OD}_{600}$ the cultures were measured without dilution.

\subsubsection{Cultivation of $E$. coli}

\subsubsection{Liquid cultivation}

The LB medium was used as a standard growth medium for E. coli cultures. To select for plasmids, it was supplemented with ampicillin (Table 3.16). Liquid E. coli cultures were inoculated with a sterile toothpick either from agar plates, short-term cultures or cryo-stocks and grown over night $(15-17 \mathrm{~h})$ at $37^{\circ} \mathrm{C}$ and $220 \mathrm{rpm}$.

\subsubsection{Short- and long-term storage}

E. coli strains were stored for 4-5 weeks on LB agar plates or in LB medium at $4^{\circ} \mathrm{C}$. For the long-term storage cryo-stocks were prepared as described for yeast strains (chap. 3.2.1.2) using 60\% (w/v) sterile glycerol. The cryo-stocks were stored at $-80^{\circ} \mathrm{C}$.

\subsection{Molecular biological methods}

\subsubsection{Isolation of chromosomal DNA from Yeast}

For the isolation of chromosomal DNA from yeast cells the NucleoSpin ${ }^{\circledR}$ Microbial DNA kit (Macherey-Nagel) was used. For this, $1.5 \mathrm{ml}$ of an overnight culture were harvested by centrifugation (13200 rpm, $1 \mathrm{~min}$ ) and further processed according to the manufacturer's instructions. 


\subsubsection{Plasmid Isolation from E. coli}

Plasmids were isolated from E. coli using the Wizard ${ }^{\circledR}$ Plus SV Miniprep System (Promega). For this, $4 \mathrm{ml}$ of an overnight culture were harvested by centrifugation (8000 rpm, $5 \mathrm{~min}$ ) and further processed according to the manufacturer's instructions.

\subsubsection{Determination of DNA concentration}

Plasmid DNA concentrations were measured using the NanoVue ${ }^{\mathrm{TM}}$ UV/Visible Spectrophotometer (GE Healthcare). For the determination of the concentration of DNA fragments (e.g. from PCR or restriction digestion) $1 \mu \mathrm{l}$ of the respective DNA sample was mixed with $5 \mu \mathrm{l}$ of the purple gel loading dye from NEB and run on a $0.8 \%$ agarose gel (chap. 3.3.4). The concentration of the DNA fragment was estimated by comparing its intensity under ultra violet (UV) light with the TriDye ${ }^{\mathrm{TM}}$ $1 \mathrm{~kb}$ DNA ladder from NEB.

\subsubsection{DNA agarose gel electrophoresis}

To analyze DNA containing samples (e.g. PCR samples or samples from restriction digestion), the DNA agarose gel electrophoresis was used as a standard method. During the electrophoresis DNA fragments were separated in the electric field according to their size. The agarose gels consisted of $0.8 \%(\mathrm{w} / \mathrm{v})$ agarose in TAE buffer (Table 3.17) and were supplemented with $1 \mu \mathrm{g} / \mathrm{ml}$ ethidium bromide for the visualization of DNA under UV light. The DNA samples were mixed with the 6x purple gel loading dye (NEB) and separated for 20-30 min at $120 \mathrm{~V}$. For size determination the TriDye ${ }^{\mathrm{TM}} 1 \mathrm{~kb}$ DNA ladder (NEB) was used as a reference. DNA bands were visualized and documented with a UV transilluminator (Biometra).

\subsubsection{DNA gel extraction}

DNA fragments were purified from agarose gels using the QIAquick Gel Extraction Kit (Qiagen) according to the manufacturer's instructions. 


\subsubsection{Polymerase chain reaction (PCR)}

The PCR was either used to generate DNA fragments for molecular cloning and homologous recombination in yeast or for the verification of gene deletions and integrations of chromosomal tags. Two deoxyoligonucleotides (primers) were designed to flank the region of interest which was amplified by a DNA polymerase during repeated cycles of heating and cooling. Depending on the required accuracy of the amplified DNA fragment, different polymerases were used. The KOD Hot Start DNA Polymerase (Merck) has a high accuracy due to its proofreading function. Therefore, it was used for the amplification of DNA fragments designated for molecular cloning or homologous recombination (50 $\mu \mathrm{l}$ scale). To evaluate the success of genomic manipulations, either a mixture of the Taq, Vent ${ }^{\circledR}$ and FideliTaq ${ }^{\mathrm{TM}}$ DNA polymerases (NEB, Affymetrix) or the DreamTaq Green PCR Master Mix (Thermo Fisher Scientific) was used (20 $\mu \mathrm{l}$ scale). The DNA fragments were either amplified from plasmid DNA or from chromosomal yeast DNA. The reactions and thermocycler programs were set up according to the manufacturer's recommendations for the respective polymerase. The thermocycler program was also adjusted to the length of the amplified fragment (elongation time) and the annealing temperature of the primers.

The amplified DNA was either purified from an agarose gel (chap. 3.3.4) or purified using the QIAquick PCR Purification Kit (Qiagen) according to the manufacturer's instructions.

\subsubsection{Restriction of DNA}

Restriction enzymes were used to analyze DNA or to generate defined DNA fragments for molecular cloning (chap. 3.3.9). Enzymes and buffers were obtained from NEB and used according to the recommendations of the manufacturer. For an analytic digestion (e.g. to verify positive plasmids after cloning) 0.5-1.0 $\mu$ g of DNA and $0.5 \mu \mathrm{l}$ of the respective enzymes were used. The reaction was incubated for 1-2 $\mathrm{h}$ at the optimal enzyme temperature and afterwards analyzed by agarose gel electrophoresis (chap. 3.3.4).

DNA fragments designated for molecular cloning were digested in a total volume of $50 \mu \mathrm{l}$. Here, 5-10 $\mu$ g of PCR product or plasmid were applied to the reaction together 
with 2-4 $\mu \mathrm{l}$ of the respective enzymes and incubated for $2-3 \mathrm{~h}$ at the recommended temperature. The desired DNA fragment was afterwards purified from an agarose gel (chap. 3.3.5).

\subsubsection{Ligation of DNA fragments}

The ligation of restriction digested DNA fragments (inserts) with linearized plasmid backbones (vectors) was performed with the T4 DNA ligase (NEB) according to the manufacturer's recommendations. To determine the optimal ratio of vector and insert, the following equation was used:

$$
\operatorname{mass}_{\text {insert }}(n g)=\frac{5 \times \text { mass }_{\text {plasmid }}(n g) \times \text { length }_{\text {insert }}(b p)}{\text { length }_{\text {plasmid }}(b p)}
$$

Typically, 150-200 ng of vector and the respective amount of insert were mixed together with $2 \mu \mathrm{l}$ of T4 DNA ligase buffer and $1 \mu \mathrm{l}$ of T4 DNA ligase in a total volume of $20 \mu \mathrm{l}$. The reaction was incubated for at least $30 \mathrm{~min}$ at room temperature or over night at $16^{\circ} \mathrm{C}$. Afterwards half of the reaction volume was transformed into chemically competent $E$. coli cells (XL1-Blue or NEB ${ }^{\circledR}$ 5-alpha) (chap. 3.3.14).

\subsubsection{Molecular cloning}

Molecular cloning was used to construct new plasmids for the expression of desired fusion proteins. For this, the insert was either amplified by PCR or isolated from another plasmid using restriction digestion (chap. 3.3.6 and 3.3.7). When amplified by PCR, the required restriction sites for cloning were introduced flanking to the insert using the designed primers (Table 3.5). After PCR, the insert was purified using the QIAquick PCR Purification Kit (Qiagen) according to the manufacturer's instructions and digested with the respective enzymes (NEB) (chap. 3.3.7). The accuracy of the PCR and the restriction digestion was analyzed by agarose gel electrophoresis and afterwards the DNA fragment was purified from the gel (chap. 3.3.4 and 3.3.5).

The vector was linearized by restriction digestion with the respective enzymes (NEB) (chap. 3.3.7). To avoid religation of the vector in the ligation reaction, $1 \mu \mathrm{l}$ of alkaline phosphatase (CIP, NEB) was added to the reaction 30 min before the end of the digestion to dephosphorylate 5'- and 3'-ends. After restriction digestion, the 
vector was also purified from an agarose gel (chap. 3.3.5). The concentration of the eluted insert and vector was determined (chap. 3.3.3) to calculate their optimal molar ratio $(5: 1)$ in the ligation reaction. To ligate vector and insert, the T4 DNA ligase (NEB) was used according to the recommendations of the manufacturer (chap. 3.3.8). After ligation, half of the volume of the reaction setup was transformed into chemically competent E. coli cells (XL1-Blue or NEB ${ }^{\circledR} 5$-alpha) (chap. 3.3.14).

\subsubsection{Plasmid construction}

Table 3.18 lists all the plasmids cloned in this study together with all necessary information about the construction of the plasmid. For more information on plasmids and primers see Table 3.3 and Table 3.5. Exemplarily, the cloning strategy of the plasmids used for the BioID assay (chap. 3.9.6) is outlined in more detail:

For the construction of pUG36-Myc-BirA*-MCS the insert Myc-BirA* was amplified by PCR using the primers Myc_BirA_XbaI_f and BirA_XbaI_rev and pFA6a-MycBirA*-TRP1 as a template. The insert and the vector pUG36 were digested with XbaI. Thereby, the yeGFP was excised from pUG36 and replaced by Myc-BirA* upon ligation of vector and insert (Figure 7.3).

As yeGFP could not be excised from the pUG35 vector as a whole by restriction digestion, it was replaced by Myc-BirA* (insert) using homologous recombination (Figure 7.3). For this, the insert was amplified by PCR using pFA6a-Myc-BirA*-TRP1 as a template. The primers pUG35_Myc_BirA_for and pUG35_BirA_rev added sequences of 40-45 nucleotides to the 3'- and 5'-ends of Myc-BirA*, that were complementary to pUG35. The vector pUG35 was linearized using SalI and PflMI, excising part of the yeGFP. Insert and vector were ligated by homologous recombination in yeast. For this, the DNA fragments were transformed together in a WT strain using the "Quick and Dirty" transformation protocol (chap. 3.3.14). Ligated plasmids of pUG35-MCS-Myc-BirA* were isolated using the plasmid rescue protocol (chap. 3.3.18) and retransformed into chemically competent E. coli XL1-blue cells (chap. 3.3.14) for amplification and further testing.

To integrate genes of interest (ATG21, ATG16) 3' or 5' to Myc-BirA* in the pUG35MCS-Myc-BirA* or pUG36-Myc-BirA*-MCS vector, the respective gene was excised 
from existing plasmids using restriction digestion and ligated into the linearized vector (Table 3.18).

Table 3.18: Plasmids cloned in this study

\begin{tabular}{|c|c|c|c|c|c|}
\hline Name & Vector & Insert & Insert origin & Primer & $\begin{array}{l}\text { Restriction } \\
\text { enzymes }\end{array}$ \\
\hline $\begin{array}{l}\text { pUG36-Myc- } \\
\text { BirA*-MCS }\end{array}$ & pUG36 & Myc-BirA* & $\begin{array}{l}\text { PCR: pFA6a-Myc- } \\
\text { BirA*-TRP1 }\end{array}$ & $\begin{array}{l}\text { Myc_BirA_XbaI_f/ } \\
\text { BirA_XbaI_rev }\end{array}$ & $X b a \mathrm{I}$ \\
\hline $\begin{array}{l}\text { pUG35-MCS- } \\
\text { Myc-BirA* }\end{array}$ & pUG35 & Myc-BirA* & $\begin{array}{l}\text { PCR: pFA6a-Myc- } \\
\text { BirA*-TRP1 }\end{array}$ & $\begin{array}{l}\text { pUG35_Myc_BirA_for/ } \\
\text { pUG35_BirA_rev }\end{array}$ & SalI, PflMI \\
\hline $\begin{array}{l}\text { pUG36-Myc- } \\
\text { BirA*-Atg21 }\end{array}$ & $\begin{array}{l}\text { pUG36- } \\
\text { Myc-BirA* }\end{array}$ & $A T G 21$ & $\begin{array}{l}\text { cloned from } \\
\text { pUG36- } \\
\text { GFP-Atg21 }\end{array}$ & 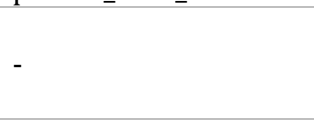 & BamHI, XhoI \\
\hline $\begin{array}{l}\text { pUG35- } \\
\text { Atg21-Myc- } \\
\text { BirA* }\end{array}$ & $\begin{array}{l}\text { pUG35- } \\
\text { Myc-BirA }\end{array}$ & ATG21 & $\begin{array}{l}\text { cloned from } \\
\text { Atg21 C-YC }\end{array}$ & - & BamHI, SalI \\
\hline $\begin{array}{l}\text { pUG36-Myc- } \\
\text { BirA* }\end{array}$ & pUG36 & Myc-BirA* & $\begin{array}{l}\text { PCR: pFA6a-Myc- } \\
\text { BirA*-TRP1 }\end{array}$ & $\begin{array}{l}\text { Myc_BirA_XbaI_f/ } \\
\text { pUG35_BirA_rev }\end{array}$ & XbaI, XhoI \\
\hline $\begin{array}{l}\text { pUG36- } \\
\text { mCherry- } \\
\text { Atg21 R151E }\end{array}$ & $\begin{array}{l}\text { pUG36- } \\
\text { mCherry }\end{array}$ & $A T G 21^{R 151 E}$ & $\begin{array}{l}\text { cloned from } \\
\text { pUG36-GFP- } \\
\text { Atg21 }\end{array}$ & 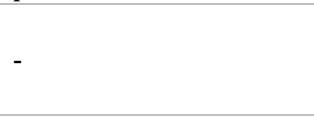 & BamHI, XhoI \\
\hline $\begin{array}{l}\text { pUG36- } \\
\text { mCherry- } \\
\text { Atg5 }\end{array}$ & $\begin{array}{l}\text { pUG36- } \\
\text { mCherry }\end{array}$ & ATG5 & PCR: Nub-Atg5 & $\begin{array}{l}\text { BamHI_Atg5_for/ } \\
\text { Atg5_XhoI_rev }\end{array}$ & BamHI, XhoI \\
\hline $\begin{array}{l}\text { pRS313- } \\
\text { PCuP1-Atg5- } \\
\text { 2xFLAG }\end{array}$ & $\begin{array}{l}\text { pRS313- } \\
\text { PCUP1 }\end{array}$ & $\begin{array}{l}\text { ATG5- } \\
\text { 2xFLAG }\end{array}$ & $\begin{array}{l}\text { PCR: pUG36- } \\
\text { mCherry-Atg5 }\end{array}$ & $\begin{array}{l}\text { SalI-Atg5_f/ } \\
\text { Atg5-2xFLAG_r }\end{array}$ & SalI, XhoI \\
\hline $\begin{array}{l}\text { pUG35- } \\
\text { Atg16-Myc- } \\
\text { BirA* }\end{array}$ & $\begin{array}{l}\text { pUG35- } \\
\text { Myc-BirA }\end{array}$ & ATG16 & $\begin{array}{l}\text { cloned from } \\
\text { pRS313-PCUP1- } \\
\text { Atg16-HA }\end{array}$ & - & EcoRI, SalI \\
\hline $\begin{array}{l}\text { pUG36-Myc- } \\
\text { BirA*-Atg16 }\end{array}$ & $\begin{array}{l}\text { pUG36- } \\
\text { Myc-BirA* }\end{array}$ & ATG16 & $\begin{array}{l}\text { cloned from } \\
\text { GST-Atg16 }\end{array}$ & - & BamHI, XhoI \\
\hline $\begin{array}{l}\text { pRS313- } \\
\text { Atg21-YFP }\end{array}$ & pRS313 & $\begin{array}{l}P_{A T G 21-} \\
\text { ATG21-YFP }\end{array}$ & $\begin{array}{l}\text { cloned from } \\
\text { pRS316-Atg21- } \\
\text { YFP }\end{array}$ & - & SacI, XbaI \\
\hline $\begin{array}{l}\text { pRS315- } \\
\text { Atg21-YFP }\end{array}$ & pRS315 & $\begin{array}{l}P_{A T G 21^{-}} \\
\text {ATG21-YFP }\end{array}$ & $\begin{array}{l}\text { cloned from } \\
\text { pRS316-Atg21- } \\
\text { YFP }\end{array}$ & - & SacI, XbaI \\
\hline $\begin{array}{l}\text { pUG23- } \\
\text { Atg18-GFP }\end{array}$ & pUG23 & $\begin{array}{l}P_{\text {ATG18- }} \\
\text { ATG18 }\end{array}$ & $\begin{array}{l}\text { PCR: } \\
\text { chromosomal } \\
\text { DNA }\end{array}$ & $\begin{array}{l}\text { SacI-PAtg18-f } \\
\text { EcoRI-Atg18ws-r }\end{array}$ & SacI, EcoRI \\
\hline $\begin{array}{l}\text { pUG23-Vac8- } \\
\text { GFP }\end{array}$ & pUG23 & $P_{V A C B^{-} V A C 8}$ & $\begin{array}{l}\text { PCR: } \\
\text { chromosomal } \\
\text { DNA }\end{array}$ & $\begin{array}{l}\text { SacI-PVac8-for/ } \\
\text { Vac8_EcoRI_rev }\end{array}$ & SacI, EcoRI \\
\hline
\end{tabular}

For the construction of pUG36-Myc-BirA* encoding only Myc-BirA*, which was needed as a control in the BioID assay, the insert Myc-BirA* was amplified by PCR using the primers Myc_BirA_XbaI_f and pUG35_BirA_rev and pFA6a-Myc-BirA*TRP1 as a template. The insert and the vector pUG36 were digested with $X b a I$ and XhoI. Thereby, the yeGFP was excised from pUG36 and replaced by Myc-BirA* upon ligation of vector and insert. 


\subsubsection{Site-directed mutagenesis of plasmids}

Point mutations were introduced into plasmids either using the QuickChange II SiteDirected Mutagenesis Kit or the QuickChange Lightning Site-Directed Mutagenesis Kit (Agilent) according to the instructions of the manufacturer. For this, complementary primers with a length of $45-55$ bp containing the desired nucleotide exchanges were designed (Table 3.7). Mutated plasmids were transformed in E. coli XL1-Blue supercompetent cells or XL10-Gold ultracompetent cells obtained together with the respective kit. The successful introduction of the point mutations was confirmed by sequencing (chap. 3.3.12).

\subsubsection{Sequencing of DNA}

Newly cloned plasmids were verified by sequencing. The sequencing was performed by Microsynth Seqlab (Göttingen). For the sequencing reaction the plasmid DNA was diluted to a concentration of $80 \mathrm{ng} / \mu \mathrm{l}$ in a total volume of $12 \mu \mathrm{l}$. To the DNA, $3 \mu \mathrm{l}$ of the respective 10 times diluted primers (Table 3.5) were added. The sequencing results were evaluated with the help of the Snap Gene software (GSL Biotech LLC).

\subsubsection{Preparation of chemically competent $E$. coli}

The $E$. coli $\mathrm{NEB}^{\circledR}{ }^{\circledR}$-alpha cells were obtained from NEB in a ready-to-use status. The E. coli XL1-Blue cells originated from the QuickChange II Site-Directed Mutagenesis Kit from Agilent and were regrown and made chemically competent by members of the group (Dr. P. Rube and L. Marquardt). The chemically competent E. coli cells were prepared according to the protocol of Hanahan (1985).

\subsubsection{Transformation of plasmid DNA in chemically competent $E$. coli}

For the transformation of plasmid DNA, the chemically competent $E$. coli cells (XL1Blue or NEB ${ }^{\circledR} 5$-alpha) were thawed on ice for $15 \mathrm{~min}$. Half the volume of the ligation reaction was gently mixed with 30-50 $\mu$ of competent cells and incubated on ice for $30 \mathrm{~min}$. The uptake of plasmid DNA was induced by heat-shock at $42^{\circ} \mathrm{C}$ for $90 \mathrm{sec}$. Afterwards, the sample was cooled-down on ice for $2 \mathrm{~min}$, filled up to $1 \mathrm{ml}$ with SOC medium and shaken for $1 \mathrm{~h}$ at $37^{\circ} \mathrm{C}$ for recovery. Finally, the cells were pelleted by centrifugation (5000 rpm, $5 \mathrm{~min}$ ) and plated on LB agar plates containing ampicillin 
for plasmid selection. The plates were incubated over night at $37^{\circ} \mathrm{C}$. Typically, several colonies were inoculated in LB medium for plasmid isolation (3.3.2). The isolated plasmids were tested for correct insertion by analytic restriction digestion and sequencing (chap. 3.3.7 and 3.3.12).

\subsubsection{High efficiency transformation of DNA in yeast}

The high efficiency transformation was used for chromosomal manipulations of yeast strains like gene deletions and the introduction of chromosomal tags. For this, $50 \mathrm{ml}$ YPD were inoculated from a stationary preculture (1:50000) and grown over night (15-17 h) to log phase. At an $\mathrm{OD}_{600}$ of 0.5-0.8 the whole culture was harvested by centrifugation (2000 rpm, $5 \mathrm{~min}$ ). The cell pellet was washed twice with sterile $\mathrm{ddH}_{2} \mathrm{O}$ and once with $2.5 \mathrm{ml} \mathrm{LiOAc-Sorb}$ buffer (Table 3.17). After the last washing step, the cell pellet was resuspended in 100-500 $\mu$ of LiOAc-Sorb buffer and incubated for $15 \mathrm{~min}$ at $30^{\circ} \mathrm{C}$. To set up the transformation reaction, $50 \mu \mathrm{l}$ of the cells were added together with $5 \mu$ l of herring sperm DNA (10 mg/ml) and $10 \mu \mathrm{l}$ of the respective DNA to $300 \mu \mathrm{l}$ of PEG in LiTE buffer (Table 3.17). The reaction was incubated for $30 \mathrm{~min}$ at $30^{\circ} \mathrm{C}$ and then heat-shocked at $42^{\circ} \mathrm{C}$ for $15 \mathrm{~min}$. For recovery, the cells were first harvested by centrifugation (2000 rpm, $5 \mathrm{~min}$ ) to remove the PEG in LiTE buffer, then shifted to $2 \mathrm{ml}$ of YPD medium and shaken for $2 \mathrm{~h}$ at $30^{\circ} \mathrm{C}$. Finally, the cells were harvested again and plated on the appropriate medium to select for the genetic markers. After $2-3$ days of incubation at $30^{\circ} \mathrm{C}$, several colonies were picked for isolation of chromosomal DNA. Gene deletion and the integration of chromosomal tags was verified by PCR (Table 3.5).

\subsubsection{Transformation of plasmid DNA in yeast ("Quick and Dirty")}

For the fast transformation of plasmid DNA into yeast cells, the short "quick and dirty" variant of the high efficiency transformation was used. For this, $300 \mu$ of the PEG in LiTE buffer (Table 3.17) were mixed with $5 \mu$ of herring sperm DNA $(10 \mathrm{mg} / \mathrm{ml})$ as well as 3-5 $\mu \mathrm{l}$ of the respective plasmid/s. With a sterile toothpick the yeast cells were directly taken from agar plates and added to the reaction. The reaction was incubated for $30 \mathrm{~min}$ at $30^{\circ} \mathrm{C}$ and then heat-shocked at $42^{\circ} \mathrm{C}$ for $15 \mathrm{~min}$. Afterwards, the cells were harvested by centrifugation (2000 rpm, $5 \mathrm{~min}$ ), 


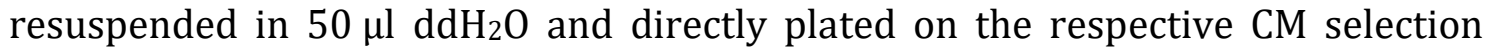
medium. The plates were incubated for $2-3$ days at $30^{\circ} \mathrm{C}$.

\subsubsection{Gene deletion and chromosomal tagging using homologous recombination}

Gene deletions and chromosomal tagging were mostly performed according to the protocol of Janke et al. (2004). In some cases also the protocol of Longtine et al. (1998) was used. For both approaches, special primers were designed built up of a $\sim 45 \mathrm{bp}$ long sequence complementary to the flanking sequences of the gene of interest and a $20 \mathrm{bp}$ long sequence complementary to the sequence of the selection cassette (e.g. hphNT1 cassette). These primers, listed in Table 3.6, were used to amplify the selection cassette from the respective plasmid (Table 3.4). The resulting DNA fragment was purified from an agarose gel and transformed into the yeast cells using the high efficiency protocol (chap. 3.3.5 and 3.3.15). Thereby, the target gene was either replaced by the selection cassette or chromosomally tagged due to homologous recombination. Potential clones were selected on the respective selection medium (e.g. YPD medium supplemented with $0.3 \mathrm{mg} / \mathrm{ml}$ hygromycin) and further verified by PCR (chap. 3.3.6).

\subsubsection{Isolation of plasmid DNA from yeast cells (plasmid rescue)}

For the isolation of plasmids from yeast cells, $1.5 \mathrm{ml}$ of an overnight culture were harvested by centrifugation (13200 rpm, $3 \mathrm{~min}$ ). The cell pellet was washed once with $500 \mu \mathrm{l}$ of $\mathrm{ddH}_{2} \mathrm{O}$ and resuspended in $200 \mu \mathrm{l}$ breaking buffer $(10 \mathrm{mM}$ Tris/HCl pH 8.0, $100 \mathrm{mM} \mathrm{NaCl}, 1 \mathrm{mM}$ EDTA, 1\% (w/v) SDS, 2\% (v/v) Triton-X-100). To the cell suspension, $200 \mu \mathrm{l}$ glass beads and $200 \mu \mathrm{l}$ phenol/chloroform solution (50\% $(\mathrm{v} / \mathrm{v})$ phenol, $50 \%(\mathrm{v} / \mathrm{v})$ chloroform) were added. The mixture was vortexed four times for $1 \mathrm{~min}$. After each vortex, the sample was incubated on ice for $1 \mathrm{~min}$. After centrifugation $\left(13200 \mathrm{rpm}, 10 \mathrm{~min}, 4^{\circ} \mathrm{C}\right.$ ), $50 \mu \mathrm{l}$ of the upper phase were transferred to a new cup. For DNA precipitation, $5 \mu \mathrm{l}$ of $3 \mathrm{M}$ sodium acetate and $140 \mu \mathrm{l}$ of ice-cold $100 \%$ ethanol were added and the mixture was incubated for $20 \mathrm{~min}$ at $-80^{\circ} \mathrm{C}$. Afterwards, the DNA was pelleted by centrifugation $\left(13200 \mathrm{rpm}, 5 \mathrm{~min}, 4^{\circ} \mathrm{C}\right)$, washed once with $200 \mu \mathrm{l}$ of ice-cold $70 \%$ ethanol and dried at room temperature 
(RT). For the transformation of the isolated plasmid into chemically competent

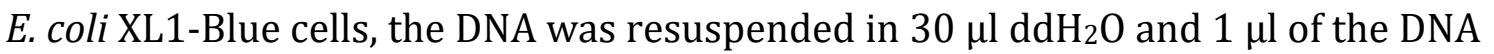
was applied to the transformation reaction (chap. 3.3.14).

\subsection{Fluorescence Microscopy}

An essential part of this study included the determination of the intracellular localization of various proteins of interest. For this, the proteins were N- or C-terminally fused to fluorescent proteins, like GFP or mCherry, which can be visualized by fluorescence microscopy. In addition, intracellular organelles as the nucleus and the vacuole were visualized using the organelle specific fluorescent dyes Hoechst and FM4-64, respectively.

For in vivo fluorescence microscopy $3 \mu$ l of a yeast culture were spotted on a microscope slide and fixed with a cover slip. If necessary, the cell density of the yeast culture was increased by centrifugation (2000 rpm, $5 \mathrm{~min}$ ) prior to its application to the microscope slide. To prevent contrast reducing reflections, the DeltaVision immersion oil ( $\mathrm{N}=1.520$, GE Healthcare) was applied to the cover slip. Fluorescence microscopy was performed with a DeltaVision ${ }^{\circledR}$ microscope (Olympus IX71, Applied Precision) equipped with a CoolSNAP HQ camera and several filter sets (Table 3.19).

Table 3.19: Overview of the used filter sets for live-cell fluorescence microscopy

\begin{tabular}{lll} 
Filter Set & Excitation wavelength (nm) & Emission wavelength (nm) \\
\hline DAPI & $390 / 18$ & $435 / 48$ \\
FITC & $475 / 28$ & $523 / 36$ \\
\hline TRITC & $542 / 27$ & $594 / 45$ \\
\hline GFP & $475 / 28$ & $525 / 50$ \\
\hline mCherry & $575 / 25$ & $632 / 69$ \\
\hline POL & $-50 / 28$ & $-50 / 0$ \\
\hline
\end{tabular}

The cells were imaged using the $100 \mathrm{x}$ objective with a $2 \times 2$ binning. A stack of at least 20 focal planes with a distance of $0.2 \mu \mathrm{m}$ was captured in a way that the whole cell was imaged from top to bottom. The resulting images were deconvolved using the softWoRx ${ }^{\mathrm{TM}}$ (Applied Precision, Issaquah, USA) software and further processed using Fiji (Schindelin et al., 2012), softWoRx ${ }^{\mathrm{TM}}$ (Applied Precision, Issaquah, USA) or the freeware mode of Huygens Professional X11 software (Scientific Volume Imaging, Hilversum, Netherlands). 


\subsection{The APE1-overexpression assay}

Suzuki et al. (2013) published this assay as a method for a detailed spatial analysis of the localization of Atg proteins at the growing phagophore using live-cell fluorescence microscopy. Here, untagged APE1 was overexpressed from the high copy pYEX-BX or pRS423 vector using the CUP1 promoter. The APE1-carrying pYEX-BX vector was provided by Prof. Dr. Kuninori Suzuki (Graduate School of Frontier Sciences, University of Tokyo). Several proteins of interest were fused to fluorescent tags to analyze their precise localization at the growing phagophore marked by GFP, mCherry or mTagBFP2 tagged Atg8.

If not stated differently, yeast main cultures were grown over night in selection medium containing $100 \mu \mathrm{M}$ copper sulfate to induce APE1-overexpression. The cells were either analyzed at stationary phase with an $\mathrm{OD}_{600}$ of 4-5 or transferred to nitrogen-free medium (SD-N) to induce autophagy. For this, $200 D_{600}$ were harvested from the main culture (at $\mathrm{OD}_{600}$ 4-5) by centrifugation (2000 rpm, $5 \mathrm{~min}$, RT). They were washed once with $2 \mathrm{ml} \mathrm{SD}-\mathrm{N}$ and finally resuspended in $2 \mathrm{ml}$ SD-N resulting in a cell density of $10 \mathrm{OD}_{600} / \mathrm{ml}$. The cells were incubated in SD-N for 1-3 $\mathrm{h}$. Fluorescently tagged proteins in the cell were visualized by fluorescence microscopy using the DeltaVision microscope (chap. 3.4).

\subsection{Fluorescence cross-correlation spectroscopy (FCCS)}

In this study, FCCS was used to monitor the molecular interaction of two target proteins in the cytosol of a yeast cell. For this, the target proteins were each tagged with one of two spectrally distinct fluorophores (GFP and mCherry). Yeast main cultures were grown to an $\mathrm{OD}_{600}$ of 1-2 and then analyzed by FCCS using a custom-made confocal microscope. FCCS measurements and calculations were performed by Dr. Narain Karedla (former member of the III. Institute of Physics, Georg-August-University Göttingen).

\subsection{Monitoring of pApe1 maturation}

The progression of the Cvt-pathway as well as unselective bulk autophagy can be monitored by the maturation of pApe1 to mApe1 after its transport to the vacuole. To analyze the progression of the Cvt-pathway under nutrient rich conditions, yeast 
main cultures were maximally grown to an $\mathrm{OD}_{600}$ of 4 before they were further processed. To monitor the progression of unselective bulk autophagy, $20 \mathrm{OD}_{600}$ were harvested from yeast main cultures at an $\mathrm{OD}_{600}$ of 4-6 by centrifugation (2000 rpm, $5 \mathrm{~min}, \mathrm{RT}$ ). The cells were washed once with $2 \mathrm{ml} \mathrm{SD-N}$ and finally resuspended in $2 \mathrm{ml}$ SD-N resulting in a cell density of $10 \mathrm{OD}_{600} / \mathrm{ml}$. The cells were incubated in SD-N for 2-4 h.

To generate crude protein extracts, $2 \mathrm{OD}_{600}$ of cells were harvested and processed according to the alkaline lysis protocol (chap. 3.9.1). The molecular weight shift of about $10 \mathrm{kDa}$ from pApe1 to mApe1 was detected by Western-Blot analysis (chap. 3.9.2) using the anti-Ape1 antibody (Table 3.8).

\subsection{Split-ubiquitin assay}

The split-ubiquitin assay was used to monitor protein-protein interactions in living cells. This technique is based on the reassembly of the $\mathrm{C}$-terminal and the $\mathrm{N}$-terminal ( $\mathrm{Cub}$ and $\mathrm{Nub}$ ) half of ubiquitin.

To determine the interaction of two proteins of interest, their genes were cloned into vectors expressing the $\mathrm{N}_{\mathrm{ub}}$ - and $\mathrm{C}_{\mathrm{ub}}-\mathrm{R}-U R A 3$-fragments from the CUP1 and MET25 promoter respectively. In detail, one gene was fused to the C-terminal part of the $\mathrm{N}_{\mathrm{ui}}$-fragment and the other to the $\mathrm{N}$-terminal part of the $\mathrm{C}_{\mathrm{ub}}$-fragment. Yeast strains were transformed with the respective $\mathrm{Nub}_{\mathrm{ub}}$ and $\mathrm{Cub}_{\mathrm{ub}}$-vectors. From a preculture of the resulting yeast strains in selection medium without His and Trp (CM-His-Trp) a cell suspension was prepared in sterile $\mathrm{dd}_{2} \mathrm{O}$ with a cell density of $1 \mathrm{OD}_{600} / \mathrm{ml}$. Based on that suspension a dilution series of 10 -fold dilutions up to 10000 was prepared in sterile $\mathrm{dd}_{2} \mathrm{O} .4 \mu \mathrm{l}$ of each dilution were dropped on three different agar plates:

The first plate consisted of selective CM medium (CM-His-Trp) and served as a growth control. The second plate consisted of selective MV medium lacking uracil (MV-His-Trp-Ura) and was supplemented with $250 \mu \mathrm{M}$ L-methionine and $100 \mu \mathrm{M}$ copper sulfate. It was used to select for negative interactions. The last plate consisted of selective CM medium (CM-His-Trp) containing $1 \mathrm{mg} / \mathrm{ml}$ 5-fluoroorotic acid (5-FOA), $250 \mu \mathrm{M}$ L-methionine and $100 \mu \mathrm{M}$ copper sulfate. It was used to select for positive interactions. The plates were incubated for two to four days at $30^{\circ} \mathrm{C}$. For 
evaluation of the growth pattern, the plates were imaged using the LAS-3000 (Fuji Photo Film).

\subsection{Biochemical methods}

\subsubsection{SDS-Polyacrylamide Gel Electrophoresis (SDS-PAGE)}

For analytical reasons, proteins were separated using the discontinuous SDS-PAGE (sodium dodecyl sulfate polyacrylamide gel electrophoresis) described in Lämmli (1970). Here, protein samples were separated according to their electrophoretic mobility which inversely correlates with the molecular weight. The SDS-polyacrylamide gels consisted of a separating (lower part; $10-12 \%$ acrylamide) and a stacking part (upper part; 5\% acrylamide) (Table 3.20).

Table 3.20: Composition of one SDS-polyacrylamide gel for a Mini-PROTEAN ${ }^{\circledR}$ III electrophoresis chamber from Bio-Rad

\begin{tabular}{lccc}
\hline \multirow{2}{*}{ Components } & \multicolumn{2}{c}{ Separating Gel } & Stacking Gel \\
& $\mathbf{1 0 \%}$ & $\mathbf{1 2 \%}$ & $\mathbf{5 \%}$ \\
\hline $\mathrm{ddH}_{2} \mathrm{O}$ & $1.95 \mathrm{ml}$ & $1.7 \mathrm{ml}$ & $3.05 \mathrm{ml}$ \\
\hline $1.5 \mathrm{M}$ Tris pH 8.8 & $1.25 \mathrm{ml}$ & $1.25 \mathrm{ml}$ & - \\
\hline $0.5 \mathrm{M}$ Tris pH 6.8 & - & - & $1.25 \mathrm{ml}$ \\
\hline Rotiphorese $^{\circledR}$ Gel $30(37.5: 1)$ & $1.75 \mathrm{ml}$ & $2 \mathrm{ml}$ & $650 \mu \mathrm{l}$ \\
\hline $10 \%(w / v)$ SDS & $50 \mu \mathrm{l}$ & $50 \mu \mathrm{l}$ & $50 \mu \mathrm{l}$ \\
\hline $10 \%(w / v)$ APS & $50 \mu \mathrm{l}$ & $50 \mu \mathrm{l}$ & $50 \mu \mathrm{l}$ \\
\hline TEMED & $2.5 \mu \mathrm{l}$ & $2.5 \mu \mathrm{l}$ & $5 \mu \mathrm{l}$ \\
\hline
\end{tabular}

SDS-PAGE was performed in Mini-PROTEAN ${ }^{\circledR}$ III electrophoresis chambers (Bio-Rad) filled with SDS running buffer (Table 3.17). The gels were loaded with 10-30 $\mu \mathrm{l}$ of protein sample dissolved in Laemmli buffer (Table 3.17) and $10 \mu \mathrm{l}$ of the Precision Plus Protein ${ }^{\mathrm{TM}}$ All Blue Prestained Protein Standard (Bio-Rad) as a reference. The electrophoresis was performed at $150 \mathrm{~V}$ until the bromophenol blue front of the Laemmli buffer reached the end of the separating gel. The separating gel was either used for Western-Blot analysis (chap. 3.9.2) or stained with Coomassie brilliant blue (chap. 3.9.3).

\subsubsection{Western-Blot analysis}

For the transfer of proteins, separated by SDS-PAGE, onto a PVDF (polyvinylidene difluoride) membrane, the semidry Western-Blotting procedure was used. For this, 
the separating gel, six blotting papers $(7 \times 10 \mathrm{~cm})$ and a PVDF membrane $(6 \times 9 \mathrm{~cm})$ were soaked in blotting buffer (Table 3.17) and arranged in the custom-made semidry blotting chamber according to the set up shown in Figure 3.1. In advance, the PVDF membrane had been incubated for $2 \mathrm{~min}$ in 100\% ethanol for activation. A maximum of two gels were blotted simultaneously in one chamber. The proteins were transferred within $90 \mathrm{~min}$ at a constant amperage of $75 \mathrm{~mA}$ per gel.

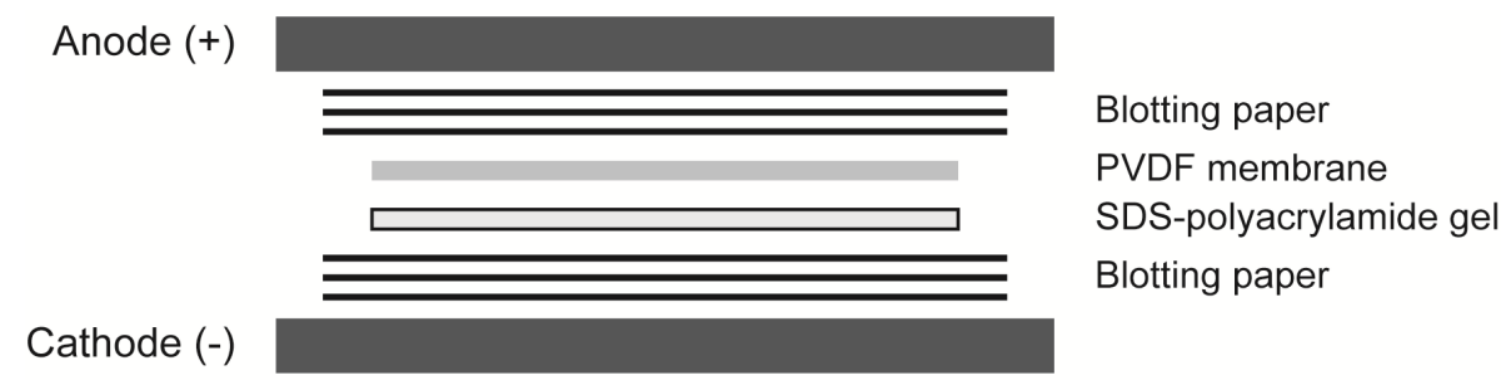

Figure 3.1: Setup of a semidry Western-Blot chamber

After the transfer process, the membranes were incubated in $25 \mathrm{ml}$ blocking buffer (10\% (w/v) skim milk powder (Granovita) in TBST) for at least $1 \mathrm{~h}$ at RT or overnight at $4^{\circ} \mathrm{C}$. Thereby, unspecific binding sites were masked by proteins of the skim milk powder. Afterwards, the blocking buffer was removed by washing the membranes three times in $25 \mathrm{ml} \mathrm{TBST}$ for $10 \mathrm{~min}$. Target proteins on the membrane were detected using specific antibodies (Table 3.8). Typically, the membranes were incubated overnight at $4^{\circ} \mathrm{C}$ with a primary antibody against the target protein. For this, the antibody was diluted in $25 \mathrm{ml}$ TBST with $1 \%(\mathrm{w} / \mathrm{v})$ skim milk powder (Granovita) (dilutions of used antibodies in Table 3.8). To remove unbound primary antibodies, the membranes were washed three times with $25 \mathrm{ml}$ TBST for $10 \mathrm{~min}$. This was followed by an $1 \mathrm{~h}$ incubation of the membranes at RT with a horseradish peroxidase (HRP)-coupled secondary antibody targeted against the primary. The HRP-coupled secondary antibody was also diluted in $25 \mathrm{ml}$ TBST with 1\% (w/v) skim milk powder (Granovita). Afterwards, the membranes were again washed with $25 \mathrm{ml}$ TBST as described above. Antibody binding was visualized using the Amersham $^{\mathrm{TM}}$ ECL $^{\mathrm{TM}}$ Western-Blotting Detection Reagents (GE Healthcare) or the Pierce $^{\mathrm{TM}}$ ECL Plus Western-Blotting Substrate (Thermo Fisher Scientific) according to the manufacturers' recommendations and depending on the amount of target protein on the membranes. The emitted light of the chemical reaction between the 
HRP and the ECL reagents was detected using the LAS-3000 (Fuji Photo Film). Detected signals were quantified using Fiji (Schindelin et al., 2012).

To remove antibodies, the membranes were incubated in $10 \%(\mathrm{v} / \mathrm{v})$ acetic acid for $10 \mathrm{~min}$ at RT. Afterwards, they were washed three times with TBST as described above, blocked in blocking buffer and subjected to another round of immunostaining.

\subsubsection{Coomassie brilliant blue (CBB) staining}

The CBB staining was used to unspecifically detect proteins in SDS-polyacrylamide gels separated by SDS-PAGE. The method used in this study was based on a protocol of Wang et al. (2007) and modified according to Pink et al. (2010). The advantage of the used CBB staining method was its compatibility with mass spectrometry.

After separation by SDS-PAGE, the proteins in the gel were fixed with fixation buffer $1(10 \%(\mathrm{v} / \mathrm{v})$ phosphoric acid, $10 \%(\mathrm{v} / \mathrm{v})$ methanol, $40 \%(\mathrm{v} / \mathrm{v})$ ethanol) for $1 \mathrm{~h}$. The gel was further incubated for $2 \mathrm{~h}$ in fixation buffer $2(1 \%(\mathrm{v} / \mathrm{v})$ phosphoric acid, $10 \%(\mathrm{v} / \mathrm{v})$ ammonium sulfate). To stain the proteins with $\mathrm{CBB}$, the gel was transferred to the staining solution $(10 \%(\mathrm{v} / \mathrm{v})$ phosphoric acid, $45 \%(\mathrm{v} / \mathrm{v})$ ethanol $0.125 \%$ CBB G 250) and incubated overnight (16 h) at RT. Background staining of the gel was reduced by incubation in destaining solution $(5 \%(\mathrm{v} / \mathrm{v})$ phosphoric acid, $40 \%(\mathrm{v} / \mathrm{v})$ ethanol) for $1 \mathrm{~h}$ and further incubation in $\mathrm{ddH}_{2} \mathrm{O}$ for up to $24 \mathrm{~h}$ until the protein bands were clearly detectable. Stained gels were imaged using a scanner and handed over to Olaf Bernhard (Department of Cellular Biochemistry, University Medical Center Göttingen) for further processing of the samples for mass spectrometry (chap. 3.10).

\subsubsection{Alkaline lysis of yeast cells}

The harsh alkaline lysis procedure was used for a fast production of crude protein extracts from yeast cells, for example to monitor the maturation of pApe1. For this, $2 \mathrm{OD}_{600}$ of yeast cells were harvested by centrifugation ( $\left.5000 \mathrm{rpm}, 5 \mathrm{~min}, \mathrm{RT}\right)$. After removal of the supernatant, the cell pellet was resuspended in $1 \mathrm{ml}$ of ice-cold water plus $150 \mu \mathrm{l}$ alkaline lysis buffer (1.85 M NaOH, 7.5\% (v/v) $\beta$-mercaptoethanol). This was followed by an incubation on ice for $10 \mathrm{~min}$. During the incubation the samples 
were mixed occasionally by harsh vortexing. For protein precipitation, $150 \mu \mathrm{l}$ of a cold 50\% (w/v) trichloroacetic acid (TCA) solution were added and the samples were thoroughly mixed. After another incubation on ice for at least $10 \mathrm{~min}$, the precipitated proteins were pelleted by centrifugation (13200 rpm, $10 \mathrm{~min}, 4^{\circ} \mathrm{C}$ ). The supernatant was removed and the pellet was washed twice with $600 \mu \mathrm{l}$ of ice-cold acetone. Finally, the pellet was air dried and resuspended in $100 \mu \mathrm{l}$ of 2x Laemmli buffer (Table 3.17). For further analysis, 15-20 $\mu$ of the samples were subjected to SDS-PAGE and Western-Blot (chap. 3.9.1 and 3.9.2).

\subsubsection{Procedure for GFP-TRAP experiments}

The GFP-TRAP approach (Chromotek) is a variant of co-immunoprecipitations where GFP-binding nanobodies fused to agarose beads are used to isolate GFPfusion proteins together with their interacting partners from cell lysates. It was used to monitor protein-protein interactions in yeast cells. For this, GFP-tagged proteins in complex with associated proteins were purified from yeast cell lysates using GFPTRAP beads (Chromotek). Here, it was especially necessary to preserve intracellular protein complexes during the lysis procedure. Therefore, cells were mildly lysed using spheroplastation in combination with osmotic shock and gentle mechanical stress.

\subsubsection{Osmotic lysis of spheroplasts}

From a $200 \mathrm{ml}$ main culture, 300-350 OD $_{600}$ of yeast cells were harvested by centrifugation (2000 rpm, $5 \mathrm{~min}, 4^{\circ} \mathrm{C}$ ) and washed once with $15 \mathrm{ml}$ of $10 \mathrm{mM} \mathrm{NaN} 3$. The cell pellet was resuspended in $3 \mathrm{ml}$ spheroplast buffer (SP-buffer; $1.4 \mathrm{M}$ sorbitol, $10 \mathrm{mM} \mathrm{NaN}_{3}, 50 \mathrm{mM} \mathrm{KH}_{2} \mathrm{PO}_{4} \mathrm{pH}$ 7.5, $40 \mathrm{mM} \beta$-mercaptoethanol) to which $100 \mu \mathrm{l}$ of a zymolyase $100 \mathrm{~T}$ solution $(0.9 \mathrm{mg} / 100 \mu \mathrm{l}$ SP-buffer $)$ were added. Beforehand, the zymolyase $\mathrm{T} 100$ solution had been shaking at $30^{\circ} \mathrm{C}$ for at least $20 \mathrm{~min}$. The cells were incubated together with the zymolyase T100 in a water bath for $30-40 \mathrm{~min}$ at $30^{\circ} \mathrm{C}$ and mild shaking (40 rpm). Afterwards, the generated spheroplasts were harvested by centrifugation $\left(2000 \mathrm{~g}, 10 \mathrm{~min}, 4^{\circ} \mathrm{C}\right)$ and washed with $3 \mathrm{ml}$ of SP-buffer. For this, the spheroplasts were carefully resuspended using a rounded glass stick while the SP-buffer was added in $1 \mathrm{ml}$ steps. The spheroplasts were again pelleted by centrifugation $\left(2000 \mathrm{~g}, 10 \mathrm{~min}, 4^{\circ} \mathrm{C}\right)$ and the supernatant was 
thoroughly removed. To lyse the cells by osmotic and mild mechanic stress, the pellet was carefully resuspended in $350 \mu$ TRAP lysis buffer (0.2 M sorbitol, 1x PBS pH 7.4, 5 mM MgCl 2,1 mM PMSF, cOmplete ${ }^{\mathrm{TM}}$ (w/o EDTA; Roche), protease inhibitor mix) and transferred to a small (size: $2 \mathrm{ml}$ ) ice-cold potter homogenizer cylinder (Sartorius). Mild mechanic stress was subjected to the spheroplasts by 2x15 pushes with the plunger. After the first 15 pushes, the potter homogenizer cylinder was cooled down on ice for $1 \mathrm{~min}$. The resulting cell lysate was transferred to a new reaction cup. The potter homogenizer cylinder was rinsed with $350 \mu$ l cold TRAP lysis buffer which were afterwards added to the cell lysate. To detach protein complexes from the membranes, $0.5 \%$ of the detergent Triton-X-100 (stock $20 \%$ (v/v) in 1x PBS pH 7.4) were added to the samples, mixed by inverting the cups 15 times and incubated on ice for $5 \mathrm{~min}$. To remove cell debris, the cell lysates were precleared by centrifugation $\left(3000 \mathrm{~g}, 10 \mathrm{~min}, 4^{\circ} \mathrm{C}\right)$. From the supernatant, $25 \mu \mathrm{l}$ were mixed with $25 \mu \mathrm{l}$ of $4 \mathrm{x}$ Laemmli buffer (Table 3.17) and boiled for $10 \mathrm{~min}$ at $95^{\circ} \mathrm{C}$ (referred to as input). The remaining supernatant was used for the GFP-TRAP.

\subsubsection{GFP-TRAP}

Prior to the application of the cell lysate, the GFP-TRAP beads (Chromotek) were equilibrated to the buffer conditions. For this, $8 \mu$ of the GFP-TRAP beads were washed once with $500 \mu \mathrm{l}$ cold 1x PBS pH 7.4, inverted 15 times and sedimented by centrifugation $\left(2700 \mathrm{~g}, 2 \mathrm{~min}, 4^{\circ} \mathrm{C}\right.$ ). The supernatant was carefully and thoroughly removed before the cell lysate was transferred to the beads. Together, they were incubated at $4^{\circ} \mathrm{C}$ and constant inverting on the Roto Shake Genie ${ }^{\circledR}$ (Scientific Industries) for 2-3 h. After incubation, the beads were harvested by centrifugation $\left(2000 \mathrm{~g}, 2 \mathrm{~min}, 4^{\circ} \mathrm{C}\right)$ and washed at least 5 times with $800 \mu \mathrm{l}$ of TRAP lysis buffer $\left(2000 \mathrm{~g}, 2 \mathrm{~min}, 4^{\circ} \mathrm{C}\right)$. Bound proteins were eluted from the beads by boiling them for $10 \mathrm{~min}$ at $95^{\circ} \mathrm{C}$ in $50 \mu \mathrm{l} \mathrm{2x}$ Laemmli buffer (Table 3.17) (referred to as bound). For further analysis, $10 \mu \mathrm{l}$ of both the input and the bound fraction of each sample were subjected to SDS-PAGE and Western-Blot analysis (chap. 3.9.1 and 3.9.2).

\subsubsection{Proximity-dependent biotin identification (BiolD) assay}

The proximity-dependent BioID assay was used to monitor protein-protein interactions or rather vicinities and to identify potential interaction partners of 
proteins of interest. Opitz et al. (2017) published a protocol for the isolation of biotinylated proteins in combination with quantitative mass spectrometry (MS) using stable isotope labeling by amino acids (SILAC). The detailed protocol was provided by Dr. Oliver Valerius and the former member Dr. Nadine Opitz (Department of Molecular Microbiology and Genetics, Georg-August-University Göttingen) and modified for the experimental needs of this study.

\subsubsection{Preparation of yeast cultures without SILAC}

To biotinylate proteins in the cell, the BirA* fusions were expressed from plasmid using the MET25 promoter. The construction of these plasmids is described in chap. 3.3.10. To prepare yeast cells for the isolation of biotinylated proteins, they were diluted 1:1000 from a preculture in $200 \mathrm{ml}$ of selection medium w/o methionine (main culture) to induce expression of the BirA* fusions. If not stated differently, the main culture was supplemented with $10 \mu \mathrm{M}$ biotin for efficient biotinylation and grown over night (15-17 h) to an OD600 of 2-3.

\subsubsection{Isolation of biotinylated proteins using Strep-Tactin ${ }^{\circledR}$ columns}

For initial method optimization and monitoring of interactions of known interaction partners (chap. 4.1.2.3 and 4.3.1), biotinylated proteins were isolated using the $0.2 \mathrm{ml}$ Gravity flow Strep-Tactin ${ }^{\circledR}$ Sepharose ${ }^{\circledR}$ Column (iba). To prepare samples for mass spectrometry, the $1 \mathrm{ml}$ Gravity flow Strep-Tactin ${ }^{\circledR}$ Sepharose ${ }^{\circledR}$ Column (iba) was used. In the following, the protocol for the $0.2 \mathrm{ml}$ columns is presented. Changes for the use of the $1 \mathrm{ml}$ column can be found in Table 3.21 .

For the isolation of biotinylated proteins, two times $400 \mathrm{OD}_{600}$ were harvested from the main culture (chap. 3.9.6.1) by centrifugation (2000 rpm, $5 \mathrm{~min}, 4^{\circ} \mathrm{C}$ ) and washed two times with $15 \mathrm{ml}$ of cold $10 \mathrm{mM}$ HEPES pH 7.9. Both cell pellets were then resuspended in $1.5 \mathrm{ml}$ of BioID lysis buffer (10 mM HEPES pH 7.9, $10 \mathrm{mM} \mathrm{KCl}$, $1.5 \mathrm{mM} \mathrm{MgCl}$, $1 \mathrm{mM}$ PMSF, $0.5 \mathrm{mM}$ DTT, cOmplete ${ }^{\mathrm{TM}}$ (w/o EDTA; Roche)) and aliquoted into four cups filled with $400 \mu \mathrm{l}$ of glass beads. The cells were lysed by harsh vortexing for $30 \mathrm{~min}$ at $4^{\circ} \mathrm{C}$. Afterwards, 4\% (w/v) SDS (stock solution: $20 \%(\mathrm{w} / \mathrm{v})$ ) were added to the lysates and mixed by inverting (15x). To denature the proteins, the samples were incubated for $10 \mathrm{~min}$ at $65^{\circ} \mathrm{C}$. The lysates were precleared of glass beads and cell debris by centrifugation (3000 g, $5 \mathrm{~min}, \mathrm{RT})$. The 
supernatant was pooled in a $15 \mathrm{ml}$-cup, mixed and cleared of remaining cell debris by centrifugation at top speed for $3 \mathrm{~min}$. From the supernatant $30 \mu \mathrm{l}$ were mixed with $30 \mu \mathrm{l}$ of $4 \mathrm{x}$ Laemmli buffer (Table 3.17) and boiled for $10 \mathrm{~min}$ at $95^{\circ} \mathrm{C}$ (referred to as input). The remaining supernatant was used for isolation of biotinylated proteins.

The $0.2 \mathrm{ml}$ Gravity flow Strep-Tactin ${ }^{\circledR}$ Sepharose ${ }^{\circledR}$ Column (iba) was prepared by removing the storage solution by gravity flow and equilibrating it with $400 \mu \mathrm{l}$ washing buffer ( $1 \mathrm{x}$ buffer W (iba), $0.4 \%$ (w/v) SDS). Afterwards, the cell lysate was applied to the column. When the lysate had completely entered the column, it was washed with $10 \mathrm{ml}$ washing buffer. Bound proteins were eluted by adding six times $100 \mu$ l elution buffer ( $1 \mathrm{x}$ buffer $\mathrm{W}$ (iba), $10 \mathrm{mM}$ biotin). All elution fractions were collected in a $1.5 \mathrm{ml}$ Protein LoBind Tube (Eppendorf).

If not stated differently, $10 \%(\mathrm{w} / \mathrm{v})$ TCA (stock solution: $50 \%(\mathrm{w} / \mathrm{v})$ ) were added to the sample to precipitate and therefore concentrate the eluted proteins. The sample was incubated on ice for $30 \mathrm{~min}$ before precipitated proteins were pelleted by centrifugation $\left(13200 \mathrm{rpm}, 10 \mathrm{~min}, 4^{\circ} \mathrm{C}\right)$. The pellet was washed two times with $500 \mu \mathrm{l}$ ice-cold acetone. Finally, it was air dried and resuspended in $60 \mu \mathrm{l}$ 2x Laemmli buffer (Table 3.17) (referred to as elution). 20-30 $\mu$ l of the sample were loaded onto $10 \%$ SDS-polyacrylamide gels and separated by SDS-PAGE for WesternBlot analysis or CBB staining (chap. 3.9.1 to 3.9.3).

For MS analysis the sample was resuspended in $30 \mu \mathrm{l} 2 \mathrm{x}$ Laemmli buffer and in total subjected to SDS-PAGE and CBB staining.

Table 3.21: List of changes in the experimental conditions using the $0.2 \mathrm{ml}$ or the $1 \mathrm{ml}$ Gravity flow Strep-Tactin ${ }^{\circledR}$ Sepharose ${ }^{\circledR}$ Column (iba)

\begin{tabular}{|c|c|c|}
\hline \multirow{2}{*}{ Experimental conditions } & \multicolumn{2}{|c|}{ Gravity flow Strep-Tactin ${ }^{\circledR}$ Sepharose ${ }^{\circledR}$ Column (iba) } \\
\hline & $0.2 \mathrm{ml}$ & $1 \mathrm{ml}$ \\
\hline $\mathrm{OD}_{600}$ harvested & $2 \times 4000_{600}$ & $2 \times 3000_{600}$ \\
\hline $\begin{array}{l}\text { Volume of BioID lysis } \\
\text { buffer }\end{array}$ & $1.5 \mathrm{ml}$ per pellet & $1.2 \mathrm{ml}$ per pellet \\
\hline $\begin{array}{l}\text { Volume of washing buffer } \\
\text { for equilibration }\end{array}$ & $0.4 \mathrm{ml}$ & $4 \mathrm{ml}$ \\
\hline $\begin{array}{l}\text { Volume of washing buffer } \\
\text { for washing }\end{array}$ & $10 \mathrm{ml}$ & $50 \mathrm{ml}$ \\
\hline Volume of elution buffer & $6 \mathrm{x} 100 \mu \mathrm{l}$ & $3 \times 1 \mathrm{ml}$ \\
\hline $\begin{array}{l}\text { Volume of } 2 \mathrm{x} \text { Laemmli } \\
\text { buffer for resuspension }\end{array}$ & $60 \mu \mathrm{l}$ & $30 \mu \mathrm{l}$ \\
\hline
\end{tabular}




\subsubsection{Preparation of yeast cultures for quantitative MS analysis}

For SILAC based quantitative MS analysis, different combinations of stable isotopes of L-arginine and L-lysine were used in a way to compare three strains in one BioID experiment (Figure 4.33).

Yeast cultures of the three strains were grown separately. The overnight (15-17 h) preculture in normal selection medium was diluted 1:100 in $10 \mathrm{ml}$ selection medium w/o methionine that was supplemented with $50 \mathrm{mg} / \mathrm{l}$ of the stable isotopes of L-arginine and L-lysine. This second preculture was grown for $7 \mathrm{~h}$ and then used to inoculate the main culture (dilution 1:1000). The main culture consisted of $100 \mathrm{ml}$ selection medium w/o methionine that was supplemented with $10 \mu \mathrm{M}$ biotin and $50 \mathrm{mg} / \mathrm{l}$ of the stable isotopes of L-arginine and L-lysine. It was grown over night (15-17 h) to an OD 600 of 2-3.

From each of the three main cultures, $200 \mathrm{OD}_{600}$ were harvested by centrifugation (2000 rpm, $10 \mathrm{~min}, 4^{\circ} \mathrm{C}$ ) and washed once with $15 \mathrm{ml} 10 \mathrm{mM}$ HEPES pH 7.9. The cell pellets were resuspended in $15 \mathrm{ml} 10 \mathrm{mM}$ HEPES pH 7.9 and the $\mathrm{OD}_{600}$ of the cell suspension was determined. According to the measured $\mathrm{OD}_{600}$ equal amounts of cells of each strain were pooled together and harvested by centrifugation (2000 rpm, $5 \mathrm{~min}, 4^{\circ} \mathrm{C}$ ). Biotinylated proteins were isolated with the $1 \mathrm{ml}$ Gravity flow Strep-Tactin ${ }^{\circledR}$ Sepharose ${ }^{\circledR}$ Column (iba) according to the protocol described above (chap. 3.9.6.2).

\subsection{Mass spectrometry (MS)}

\subsubsection{Sample preparation}

Samples, derived from the BioID assay (chap. 3.9.6), were separated on a $10 \%$ SDSpolyacriylamide gel by SDS-PAGE. The separation was stopped when the bromophenol blue front of the sample had passed approx. $3 \mathrm{~cm}$ of the separating gel. The proteins were stained using the CBB staining method (chap. 3.9.3). In-gel digestion with trypsin as well as subsequent purification of the derived peptides by C18-Stage-tipping in preparation for MS analysis was performed by Olaf Bernhard (Department of Cellular Biochemistry, University Medical Center Göttingen) according to Opitz et al. (2017). For the in-gel digestion with trypsin, the sample lane 
(length approx. $3 \mathrm{~cm}$ ) was divided into 10 sections (Figure 4.33). Each section was further cut into approx. 2 × $2 \mathrm{~mm}$ sized gel pieces.

\subsubsection{Liquid chromatography-mass spectrometry (LC-MS) analysis}

The prepared peptide pellets (vacuum dried) were resuspended in $20 \mu \mathrm{l}$ of fresh LC-MS sample buffer (2\% (v/v) acetonitrile, $0.1 \%(\mathrm{v} / \mathrm{v})$ formic acid) by pipetting up-and-down (10x) and incubating it in an ultrasonic bath for $3 \mathrm{~min}$. LC-MS analysis for protein identification and SILAC-based protein quantification was performed by Dr. Oliver Valerius and Dr. Kerstin Schmitt (Department of Molecular Microbiology and Genetics, Georg-August-University Göttingen) with a Q exactive ${ }^{\mathrm{TM}}$ HF Hybrid Quadrupol-Orbitrap ${ }^{\mathrm{TM}}$ mass spectrometer (Thermo Fisher Scientific).

Protein and biotin-site identification as well as SILAC-based quantification was performed by Dr. Kerstin Schmitt with the Proteome DiscovererTM 2.2 software (Thermo Fisher Scientific). The SequestHT and Mascot search algorithms were used for database searches against a S. cerevisiae specific database (SGD, 6110 entries including common contaminants, S288C_ORF_database release version 2011, Stanford University).

\subsection{Statistical analysis}

To plot and statistically evaluate the data obtained from Western-Blot quantifications or fluorescence microscopy, GraphPad Prism ${ }^{\circledR} 7$ (GraphPad Software, La Jolla, USA) was used. Graphs were plotted using the mean value together with the error of the mean (SEM, depicted by error bars).

The statistical relevance was determined either with the one sample t-test or the unpaired two-tailed t-test. The one sample t-test was used to evaluate in a set of normalized data, where one sample was set to a fixed value (for example 100\%), whether the other samples significantly differ from the fixed sample. Instead, with the two-tailed t-test two samples were directly compared to determine if there was a significant difference between them. The statistical relevance is indicated in the graphs as follows: not significant for $\mathrm{P}>0.05$ (ns or no asterisk), * for $\mathrm{P}<0.05$, ${ }^{* *}$ for $\mathrm{P}<0.01,{ }^{* * *}$ for $\mathrm{P}<0.001$ and ${ }^{* * * *}$ for $\mathrm{P}<0.0001$. 


\section{Results}

\subsection{Detailed analysis of the interaction of Atg21 and Atg16}

Juris et al. (2015) showed that Atg21 organizes PI3P-dependent the covalent coupling of Atg8 to PE by recruiting Atg8 and the Atg12 Atg5/Atg16 complex to the PAS. Here, Atg21 directly interacts with the coiled-coil domain (CCD) of Atg16 (Juris et al., 2015).

One main goal of this study was a detailed analysis of the interaction between Atg21 and Atg16, to gain further insights into the molecular function and dynamics of the Atg8 lipidation. Therefore, the interacting domains of Atg21 and Atg16 were further characterized. Amino acids potentially contributing to the interaction were identified, mutated by site-directed mutagenesis and their influence on the progression of the Cvt-pathway, the binding of Atg21 and Atg16 as well as the localization of Atg16 was analyzed. The dynamics of the complex formation between Atg21 and the Atg12 Atg5/Atg16 complex were addressed by performing fluorescence cross-correlation spectroscopy (FCCS) measurements with a lipid-binding deficient mutant of Atg21. Different experimental approaches were tested, to support the resulting data.

\subsubsection{Fine mapping of the interacting domains of Atg21 and Atg16}

\subsubsection{Identification of amino acids that potentially contribute to the interaction of Atg21 and Atg16}

Ongoing from the work of Juris et al. (2015), the cooperation partners Dr. Karin Kühnel and Dr. Janina Metje (former members of the Department of Neurobiology, Max Planck Institute for Biophysical Chemistry, Göttingen) crystallized recombinantly expressed and purified Kluyveromyces lactis Atg21 (KlAtg21) together with the CCD of Ashbya gossypii Atg16 (AgAtg16; 40-124). The structure of the complex was solved up to a resolution of $4.0 \AA$ by molecular replacement with the known structures of Pichia angusta Atg18 (PaAtg18; PDB code: 5LTD and 5LTG, Scacioc et al., 2017) and Saccharomyces cerevisiae Atg16 (ScAtg16; PDB code: 3A7P, Fujioka et al., 2010). In the crystal structure two molecules of KlAtg21 bind to the 
CCD of AgAtg16 (Figure 4.1). KlAtg21 exhibits the typical PROPPIN fold as a seven-bladed $\beta$-propeller. The two KlAtg21 molecules bend with the upper part of their bottom side towards the CCD of AgAtg16, thereby adopting a reverse V shape. In this conformation the C-terminal part of the CCD of AgAtg16 is in close proximity to the blades 1, 2 and 3 on the bottom side of the KlAtg21 propeller (Figure $4.1 \mathrm{~B}$ ). The conserved membrane binding motif FRRG is located on the $\beta$-strand 5D and the connecting loop to $\beta$-strand 6A facing away from AgAtg16 (Figure 4.3, Metje, 2017).
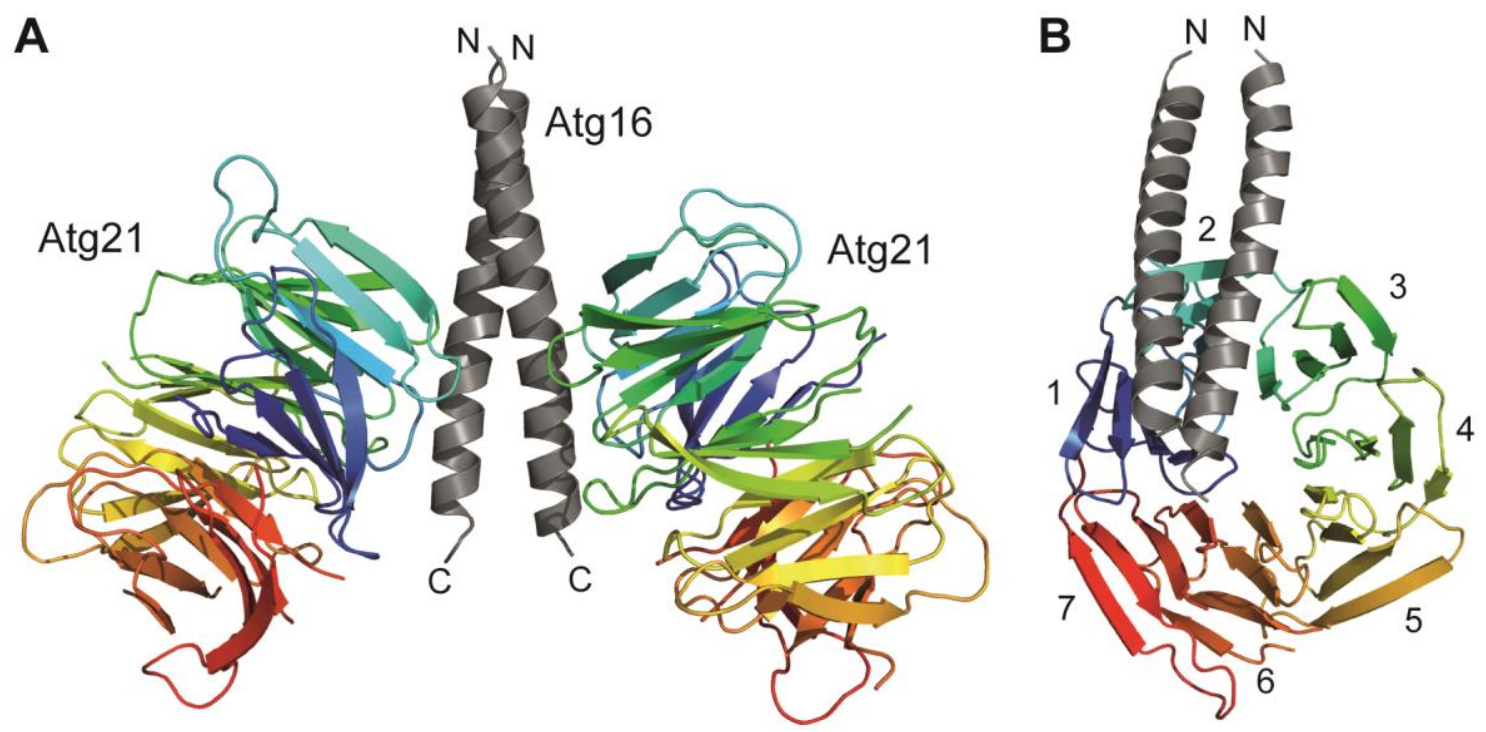

Figure 4.1: Overview of the crystal structure of KlAtg21 in complex with AgAtg16 (40-124)

Cartoon representation of KlAtg21 rainbow colored with blue to red from the $\mathrm{N}$ - to the C-terminus and of AgAtg16 in grey. The crystal structure was solved by Dr. Karin Kühnel and Dr. Janina Metje up to a resolution of $4.0 \AA$ A. (A) In the crystal structure two KlAtg21 molecules form a complex with the C-terminal part of the AgAtg16 (40-124) coiled-coil domain. (B) View onto the bottom side of one of the KlAtg21 propellers. The AgAtg16 coiled-coil domain is located in close proximity to blades 1,2 and 3 of the KlAtg21 $\beta$-propeller. Blades are numbered from 1 to 7 from $\mathrm{N}$ - to $\mathrm{C}$-terminus.

Due to the low $4.0 \AA$ A resolution of the data, side chains are not clearly visible in the electron densities of the crystal structure. Therefore, the amino acids of the proteins were built-in as alanines (Metje, 2017). From the orientation of KlAtg21 in the crystal those loops and $\beta$-strands could be identified that are in proximity to the AgAtg16 CCD and therefore might be involved in its binding (Table 4.1). The orientation of the side chains of those amino acids forming the respective loops could not be observed due to resolution limits.

Dooley et al. (2014) showed that two arginine residues (R108 and R125) are important for the interaction of the human orthologue WIPI2b (hWIPI2b) with human Atg16L1 (hAtg16L1). One of these residues (R108) is highly conserved among PROPPIN-family members (Figure 4.2, red arrow, Wilson et al., 2014). This 
suggested that the interaction mechanism of PROPPINs with Atg16 might be conserved from yeast to human.
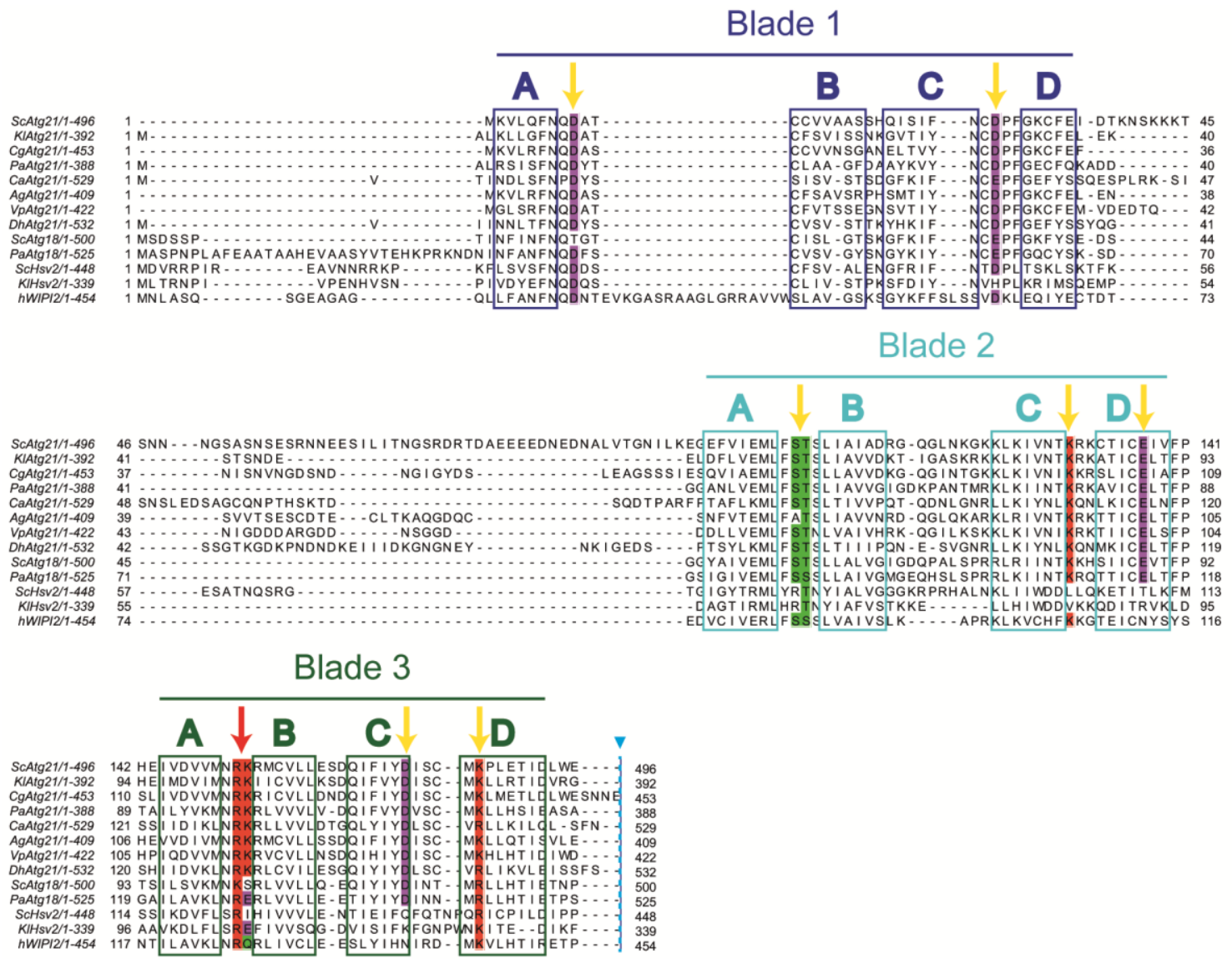

Figure 4.2: Sequence alignment of yeast and fungal PROPPINs together with the human orthologue WIPI2

Comparison of the protein sequences of ScAtg21 (Saccharomyces cerevisiae; Q02887), KlAtg21 (Kluyveromyces lactis; Q6CLZ2), CgAtg21 (Candida glabrata; Q6FRU4), PaAtg21 (Pichia angusta; Q5QJC0), CaAtg21 (Candida albicans; Q5AI22), AgAtg21 (Ashbya gossypii; Q75D34), VpAtg21 (Vanderwaltozyma polyspora; A7TTC8), DhAtg21 (Debaryomyces hansenii; Q6BIA1), ScAtg18 (S. cerevisiae; P43601), PaAtg18 (P. angusta; Q5QA94), ScHsv2 (S. cerevisiae; P50079), KlHsv2 (K. lactis; Q6CN23) and hWIPI2 (human; Q9Y4P8). The sequences were aligned with Mafft and plotted using JalView (Waterhouse et al., 2009). Sequences, sequence numbering and protein identifiers (in parentheses) were obtained from UniProt. Selected columns belonging to D9, D28, S105, T106, K130, E137, R151, K152, D167 and K172 of ScAtg21 were colored using the Clustal color scheme (yellow and red arrows). To reduce the size of the figure, sequences after the third blade of the propellers are hidden (depicted by inverted triangle and dotted line in cyan). The four $\beta$-strands of each blade are boxed and labeled from $A$ to $D$.

Based on this hypothesis, conserved amino acids were selected (Figure 4.2, yellow arrows) that are located in the loops and $\beta$-strands which are in close proximity of the AgAtg16 CCD (Table 4.1, Figure 4.3). Especially positively and negatively charged amino acids were chosen since a binding mechanism via charged amino acids was reported for hWIPI2b and hAtg16L1 (Dooley et al., 2014). Furthermore, it was shown that the negatively charged amino acids D101 and E102 of Atg16 
directly interact with Atg21 (Juris et al., 2015). In the crystal structure these residues of Atg16 are in close proximity to R151 and the neighboring K152 of Atg21 (Figure 4.3 B). In addition, ScAtg21 R151 turned out to be the counterpart of hWIPI2b R108 (Figure 4.2, red arrow, Wilson et al., 2014). In the crystal structure, a distance of $6.7 \AA$ was measured between the respective alanine side chains of Atg16 D101 and Atg21 R151 (Figure 4.3 B). This distance might be even smaller considering that both, arginine and aspartic acid, have significantly longer side chains than the alanines in the model. Therefore, the side chains could be within a distance range of $4 \AA$ which would qualify them to form a salt bridge (Kumar and Nussinov, 2002).

Table 4.1: List of the identified amino acids potentially contributing to the interaction of Atg21 and Atg16

\begin{tabular}{|c|c|c|c|c|}
\hline Protein & Loop of Atg21 & Amino acid & Mutated to & Reference \\
\hline \multirow{8}{*}{$\operatorname{Atg} 21$} & $1 \mathrm{AB}$ & D9 & $\mathrm{R}$ & \\
\hline & $1 \mathrm{CD}$ & D28 & $\mathrm{R}$ & \\
\hline & $2 \mathrm{AB}$ & S105 T106 & A & \\
\hline & \multirow{2}{*}{$2 \mathrm{CD}$} & K130 & E & \\
\hline & & E137 & $\mathrm{R}$ & \\
\hline & $3 \mathrm{AB}$ & R151 K152 & $\mathrm{E}$ & (Dooley et al., 2014) \\
\hline & \multirow{2}{*}{$3 C D$} & D167 & $\mathrm{R}$ & \\
\hline & & K172 & $\mathrm{E}$ & \\
\hline \multirow{4}{*}{$\operatorname{Atg} 16$} & & K78 & $\mathrm{E}$ & \\
\hline & & E81 & $\mathrm{R}$ & \\
\hline & & K94 & $\mathrm{E} / \mathrm{A}$ & \\
\hline & & D101 E102 & $\mathrm{R}$ & (Juris et al., 2015) \\
\hline
\end{tabular}

Besides the already published D101 and E102 of Atg16, additional amino acids were selected for the analysis of their influence on the interaction between Atg21 and Atg16 (Table 4.1). Here, it was important not to choose amino acids contributing to the stability of the CCD formation (Fujioka et al., 2010). Atg16 K94 was conserved among yeast homologs of Atg16 (Fujioka et al., 2010) and also located close to the loop 3CD of Atg21 in the crystal structure. Atg16 K78 and E81 were selected as a control since they were neither conserved (Fujioka et al., 2010), nor found to be in proximity to Atg21 in the crystal structure (Figure 4.3).

The influence of all selected amino acids on the formation of the Atg21 and Atg16 complex was analyzed by mutating them preferentially into oppositely charged residues using site-directed mutagenesis (Table 4.1). Several in vivo and in vitro 
experiments were performed to analyze the effect of these mutations (chap. 4.1.1.2 and 4.1.1.3).

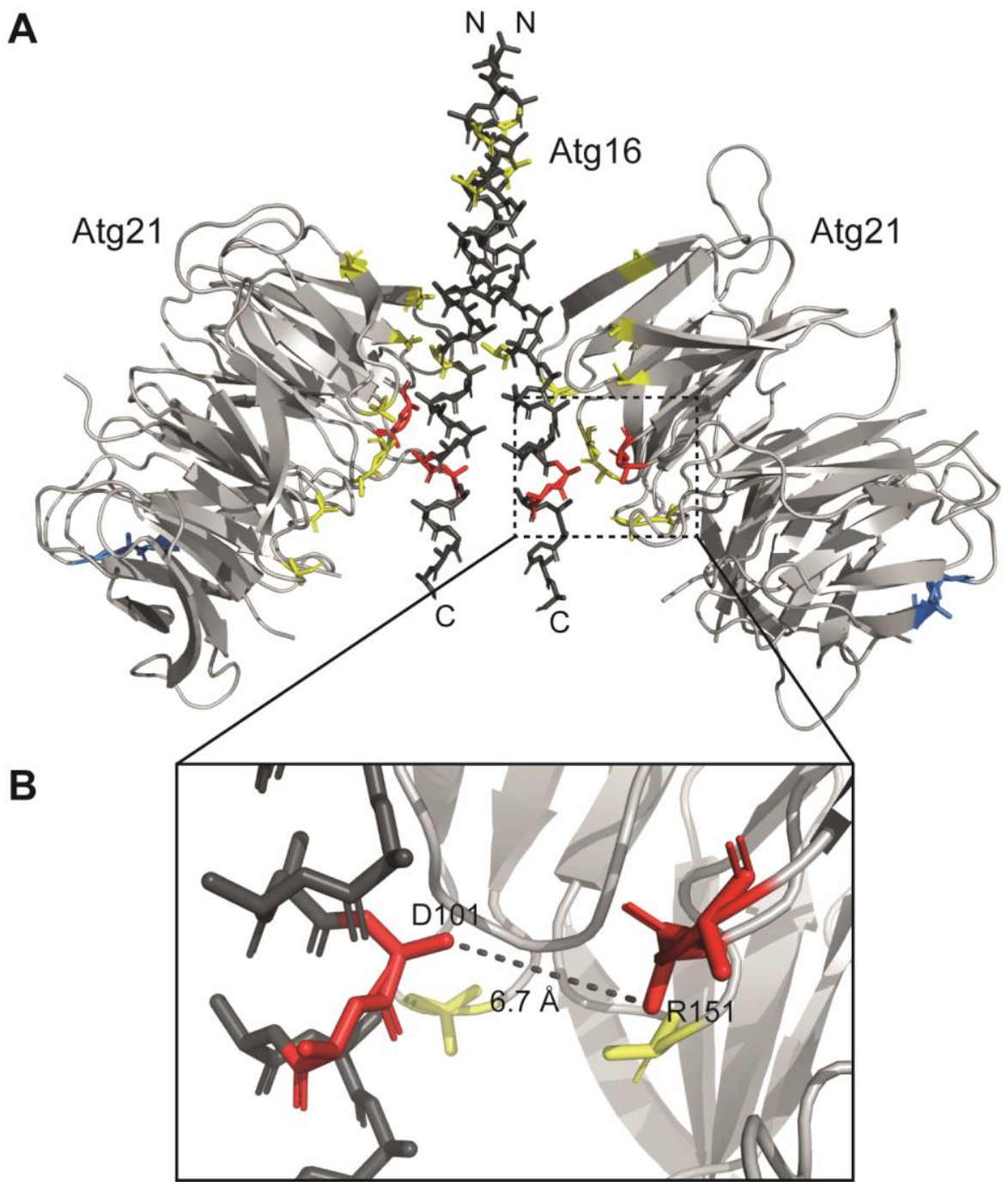

Figure 4.3: Overview of the potentially interacting amino acids of Atg21 and Atg16 in the crystal structure of KlAtg21 and AgAtg16 (40-124)

Stick representation of the AgAtg16 CCD in grey and cartoon representation of KlAtg21 in light grey. (A) Identified amino acids potentially contributing to the interaction of Atg21 and Atg16 are colored in yellow and red and are shown as sticks. They face away from the conserved lipid-binding motif FRRG which is represented as sticks in blue. Atg21: Yellow amino acids were identified due to their localization in the crystal structure and their conservation among yeast Atg21 homologs. They are all located on loops and $\beta$-strands of the blades 1 to 3 and directed towards the bottom side of the propeller and the coiled-coil domain of Atg16. Red amino acids were identified due to their published role in the interaction of the human orthologues WIPI2b and Atg16L1 (Dooley et al., 2014). Atg16: Yellow amino acids were chosen due to localization and conservation or served as controls. Red amino acids were shown to influence the interaction with Atg21 (Juris et al., 2015). (B) Enlarged view on the red colored amino acids. The dotted line shows the distance between the side chains of the modeled alanines of Atg16 D101 and Atg21 R151 (6.7 ̊̊). 


\subsubsection{R151 of Atg21 directly interacts with D101 of Atg16}

Due to the reported role of hWIPI2b R108 in Atg16L1 interaction, the corresponding amino acid in Atg21, R151, was the most promising candidate for the interaction site of Atg16. Since the neighboring K152 was also conserved among Atg21 homologs, it could possibly support or take over the function of R151. Therefore, both residues were mutated into oppositely charged amino acids (aspartic acids) in an GFP-ATG21 expressing plasmid.

Since Atg21 is essential for the Cvt-pathway the effect of the mutations on its progression was analyzed by Western-Blot analysis (Figure 4.4 A). GFP-ATG21 as well as the R151E and K152E single and double mutants were expressed from the MET25 promoter in an atg21 strain. As a positive control the WT strain and as respective negative controls, in which the Cvt-pathway is blocked, the $\operatorname{atg} 21 \Delta$ and the $\operatorname{atg} 1 \Delta$ strains were transformed with an empty pUG36 vector expressing only the GFP-tag. The Cvt-pathway acts under nutrient rich conditions, therefore the cells were grown to stationary phase (OD600 4). The progression of the Cvt-pathway was evaluated by determining the ratio of mApe1 to the total amount of Ape1 in the cell (pApe1 maturation rate). The pApe1 maturation rate of the WT was set to $100 \%$ (Figure 4.4 B).

As expected, the pApe1 maturation was blocked in the $\operatorname{atg} 21 \Delta$ and the $\operatorname{atg} 1 \Delta$ strain. They showed a pApe1 maturation rate of less than 5\% compared to the WT. Remarkably, the GFP-Atg21 fusion protein almost fully restored the function of the Cvt-pathway $(89.17 \pm 2.3 \%$ of WT). Probably, the GFP-tag or expression level somewhat affects the functionality of Atg21. This must be considered for all following experiments. Nevertheless, the pApe1 maturation rate of the GFP-ATG21 expressing strains was set to 100\% (WT) in all following experiments for a better comparison to the analyzed mutants (Figure 4.6 and Figure 4.8).

The mutation of R151E significantly reduced the pApe1 maturation rate to $55.56 \pm 3.58 \%$ compared to the WT. In contrast, the single mutant K152E did not affect the progression of the Cvt-pathway. The pApe1 maturation rate $(90.99 \pm 3.7 \%$ of WT) was comparable to that of the GFP-Atg21 WT $(89.17 \pm 2.3 \%$ of WT $)$, although the mutant seemed to be less stable than the WT protein. The combination of both mutations (R151E and K152E) highly destabilized the protein and almost blocked 
the pApe1 maturation $(7.63 \pm 3.21 \%$ of WT). Most likely the reduced stability of the protein was responsible for the loss of pApe1 maturation rather than the mutation of K152E. This suggested that mainly R151 of Atg21 is essential for the Cvt-pathway.
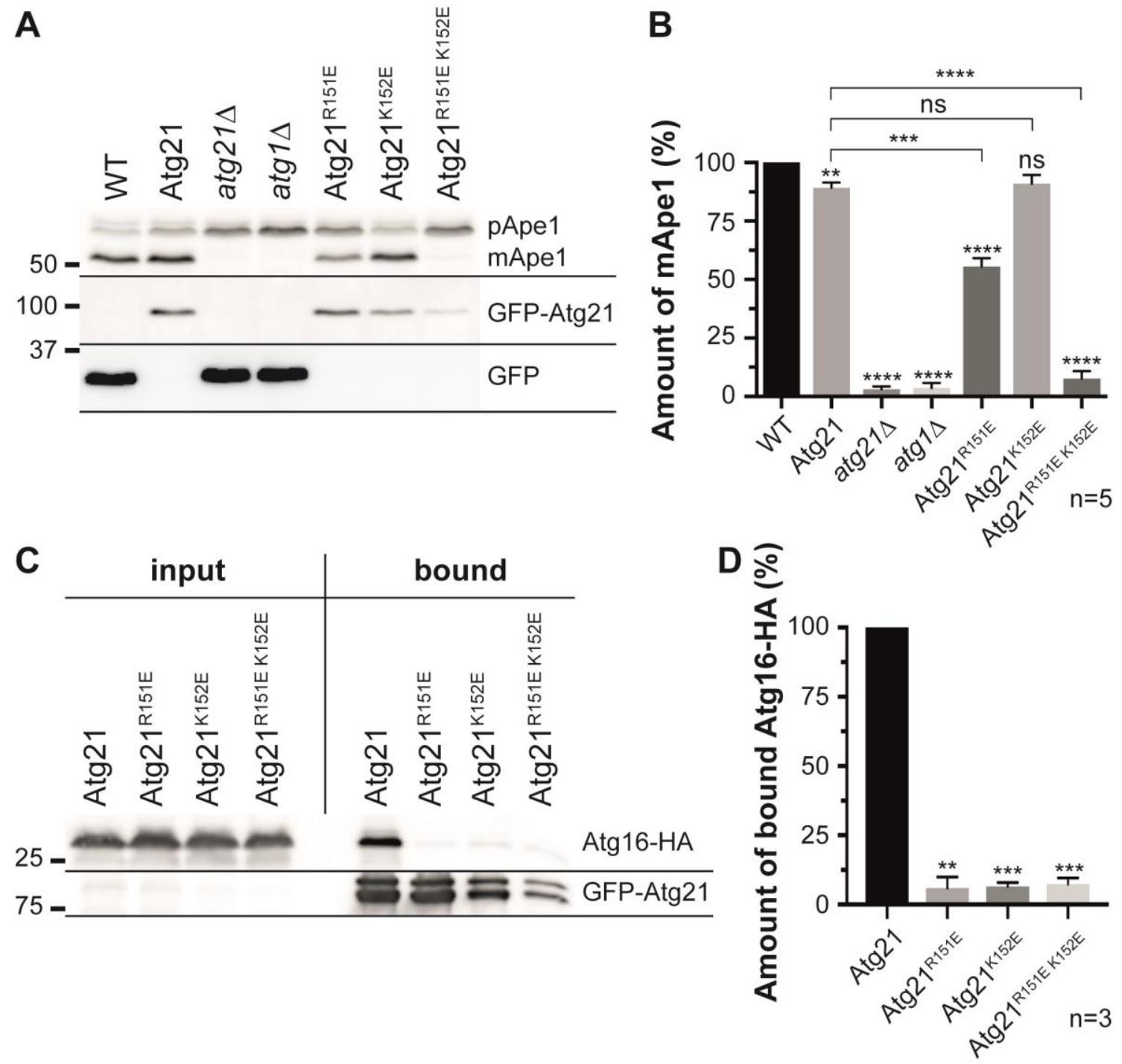

Figure 4.4: Effect of the Atg21 mutations R151E and K152E on the Cvt-pathway and the interaction with Atg16

(A) Analysis of the effect of the Atg21 R151E and K152E single and double mutants on the maturation of pApe1. In an atg21 strain plasmid-encoded GFP-ATG21 and its mutants were expressed using the

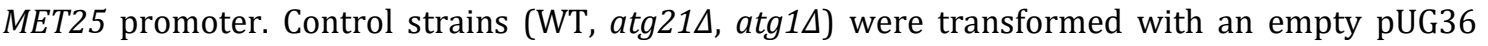
vector expressing only GFP. Cells were grown over night in selection medium without L-methionine to stationary phase $\left(\mathrm{OD}_{600} 4\right)$, alkaline lysed and analyzed by Western-Blot. The molecular weight marker is depicted in $\mathrm{kDa}$. (B) Quantification of the pApe1 maturation rate (ratio of mApe1 to the total amount of Ape1) measured in Western-Blot analysis of five independent experiments (n). WT was set to $100 \%$. Statistical relevance was determined using the one sample t-test. Atg21 and its mutants were compared using the unpaired two-tailed t-test. (C) Analysis of the effect of the Atg21 R151E and K152E single and double mutants on the interaction with Atg16 using the GFP-TRAP approach. In an $\operatorname{atg} 16 \Delta$ strain the plasmid encoded bait (GFP-ATG21 or its mutants) and prey (ATG16-HA) were overexpressed using the MET25 and CUP1 promoter respectively. Cells were grown in selection medium without L-methionine to early stationary phase ( $\mathrm{OD}_{600} 2$ ), osmotically lysed and incubated with GFP-TRAP beads. Input and bound fractions were analyzed by WesternBlot. (D) Quantification of three independent GFP-TRAP experiments (n). The amount of bound 
Atg16-HA was normalized to its amount in the input fraction and the amount of bound GFP-Atg21. The normalized amount of Atg16-HA bound to the GFP-Atg21 WT was set to $100 \%$. Statistical relevance was determined using the one sample t-test. (B and D) Error bars indicate SEM and asterisks indicate $\mathrm{p}$-values: ns, not significant $P>0.05$; ${ }^{* *} P<0.01$; ${ }^{* * *} P<0.001$; ${ }^{* * * *} P<0.0001$.

GFP-Atg21 was able to bind Atg16. This was shown by Juris et al. (2015) and confirmed in this study using the GFP-TRAP approach (Figure 4.4 C). In addition, the effect of the R151E and K152E mutants on the interaction of Atg21 and Atg16 was analyzed. Therefore, plasmid-encoded GFP-Atg21 and its mutants were overexpressed together with ATG16-HA in an atg16 $\triangle$ strain using the MET25 and the CUP1 promoter respectively. Cells were grown to early stationary phase $\mathrm{OD}_{600}$ 2) in selection medium without L-methionine. No additional copper sulfate was added to the medium to further induce the CUP1 promoter as the expression of ATG16-HA was sufficient in normal medium. The cells were spheroplasted and osmotically lysed. GFP-Atg21 containing complexes were isolated from the resulting cell extracts using GFP-TRAP beads (Chromotek) (chap. 3.9.5).

While Atg16-HA was efficiently co-isolated together with GFP-Atg21, the single and double mutation of Atg21 R151E and K152E caused an almost complete loss of interaction with Atg16 (Figure 4.4 C and D). The amount of Atg16-HA isolated in complex with the mutants was less than $10 \%$ of the amount isolated with GFP-Atg21. In contrast to the unaffected pApe1 maturation, the stability of the complex of Atg21 and Atg16 was significantly reduced for GFP-Atg21 comprising only the K152E mutation. Both, R151 and K152, seemed to contribute to the interaction of Atg21 and Atg16.

Due to the close proximity of Atg21 R151 and K152 to Atg16 D101 and E102 in the crystal structure (Figure 4.3), a direct interaction between the residues was very likely. Juris et al. (2015) already confirmed a loss of interaction between Atg21 and Atg16 after mutating D101 and E102 of Atg16 to alanine. If there was a salt bridge formed between Atg21 and Atg16, the mutation of one of the participating amino acids should disturb the interaction while the mutation of both participating amino acids into oppositely charged residues should restore it. Such a charge change experiment was already successfully used to demonstrate the direct interaction between hWIP2b R108 and hAtg16L1 E230 (Dooley et al., 2014). Therefore, both D101 and E102 of Atg16 were mutated into oppositely charged amino acids (arginines) in the ATG16-HA expressing plasmid. GFP-TRAPs were performed to 
analyze if any combination of the single and double mutants of Atg16 with those of Atg21 restored their interaction (Figure 7.1 and Figure 4.5).
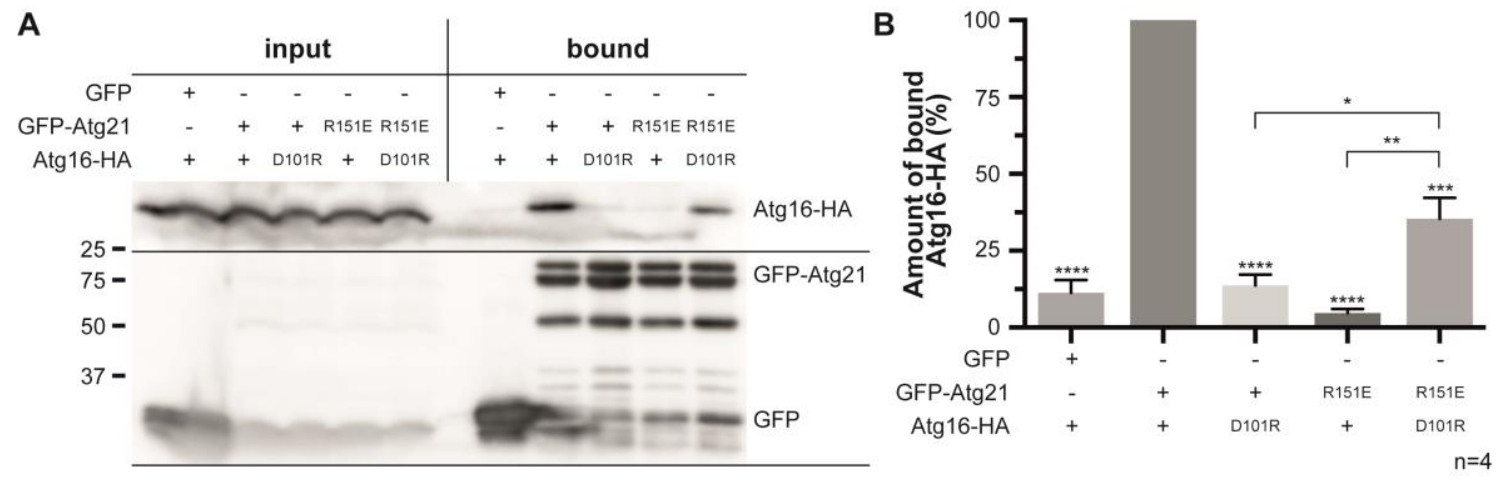

Figure 4.5: Simultaneous charge change of Atg21 R151 (R151E) and Atg16 D101 (D101R) partially restores the interaction of both proteins

(A) Analysis of the effect of the Atg21 R151E mutant on the interaction with Atg16 and the Atg16 D101R mutant using the GFP-TRAP approach. In an $\operatorname{atg} 21 \Delta$ atg16 $\Delta$ strain the plasmid-encoded bait (GFP, GFP-ATG21 or its R151E mutant) and prey (ATG16-HA or its D101R mutant) were overexpressed using the MET25 and CUP1 promoter respectively. Cells were grown in selection medium without L-methionine to early stationary phase $\left(\mathrm{OD}_{600} 2\right)$, osmotically lysed and incubated with GFP-TRAP beads. Input and bound fractions were analyzed by Western-Blot. The molecular weight marker is depicted in $\mathrm{kDa}$. (B) Quantification of four independent GFP-TRAP experiments (n). The amount of bound Atg16-HA or Atg16 D101R-HA was normalized to its amount in the input fraction and the amount of bound GFP-Atg21 or GFP-Atg21 R151E. The normalized amount of Atg16HA bound to GFP-Atg21 WT was set to $100 \%$. Statistical relevance was determined using the one sample t-test. Different combinations of WT proteins and mutants were compared using the unpaired two-tailed t-test. Error bars indicate SEM and asterisks indicate p-values: ${ }^{*} P<0.05$; ${ }^{* *} P<0.01$; ${ }^{* * *}$ $P<0.001 ;{ }^{* * * *} P<0.0001$.

For all combinations of the Atg16 E102R mutant and the D101R E102R double mutant with either of the Atg21 mutants, a loss of interaction was observed in the performed GFP-TRAP experiments (Figure 7.1). This was also true for the combination of the Atg16 D101R mutant with the Atg21 K152E mutant and the R151E K152E double mutant (Figure 7.1). However, when combining the Atg16 D101R mutant with the Atg21 R151E mutant the interaction of both proteins was partially restored (Figure 4.5). Compared to the WT proteins, only $35.25 \pm 6.9 \%$ of Atg16 D101R-HA was isolated in complex with GFP-Atg21 R151E (Figure 4.5 B). However, this was significantly more than the amount of Atg16-HA isolated when only Atg16 D101R (13.67 $\pm 3.58 \%$ of WT) or Atg21 R151E (4.74 $\pm 1.27 \%$ of WT) were mutated or when GFP alone was expressed as a bait (11.26 $\pm 4.16 \%$ of WT). GFP alone served as a control to detect if there was unspecific binding of Atg16-HA to the GFP-tag of Atg21.

The effect of the restored interaction between the Atg16 D101R and Atg21 R151E mutant was even more obvious when analyzing the progression of the Cvt-pathway. 
Therefore, ATG16-HA or its D101R mutant was expressed in combination with GFP-ATG21 or its single and double mutants (R151E and K152E) in an $\operatorname{atg} 21 \Delta \operatorname{atg} 16 \Delta$ strain. For all combinations, the ratio of mApe1 to the total amount of Ape1 in the cell was measured in Western-Blot analysis (Figure 4.6).

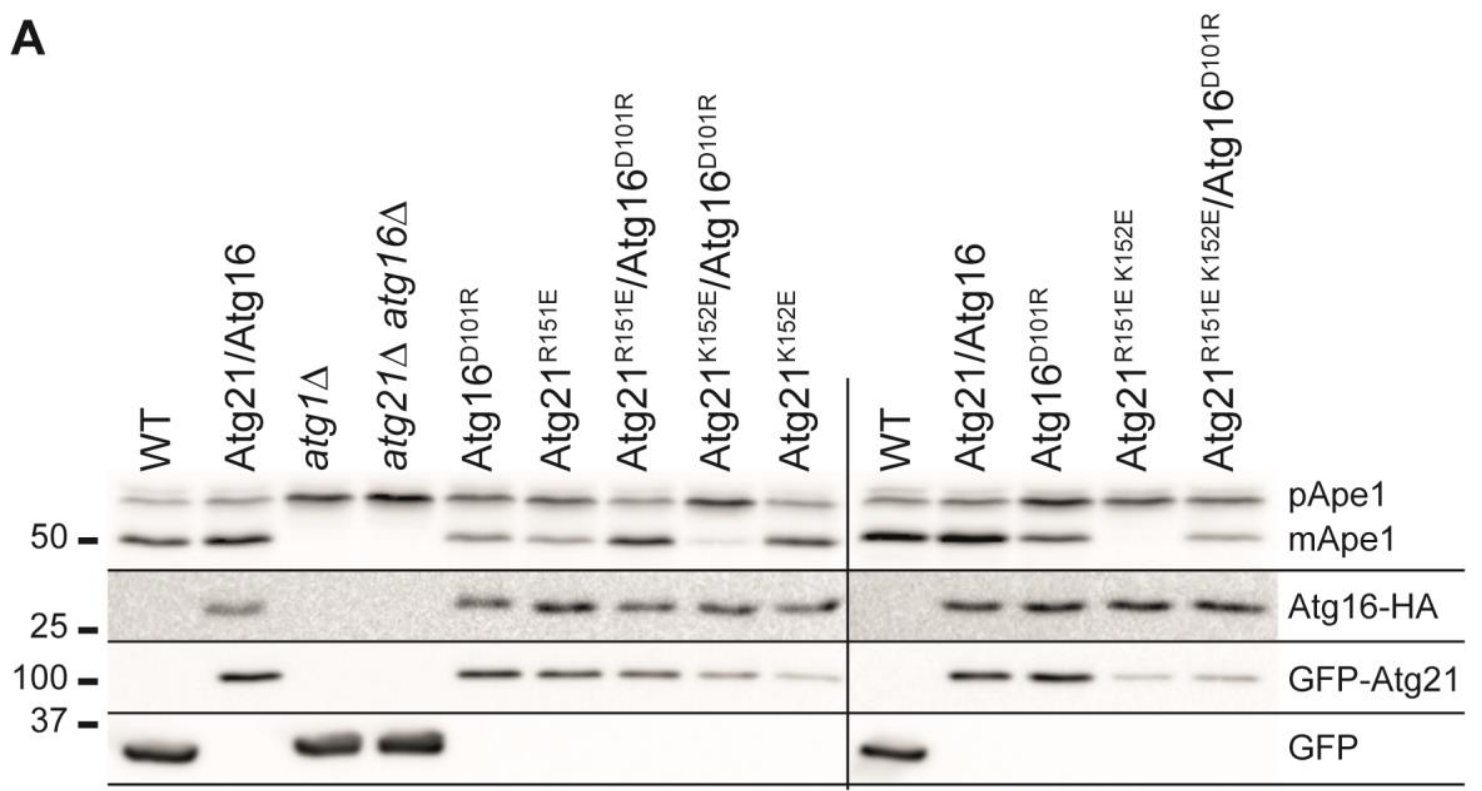

B

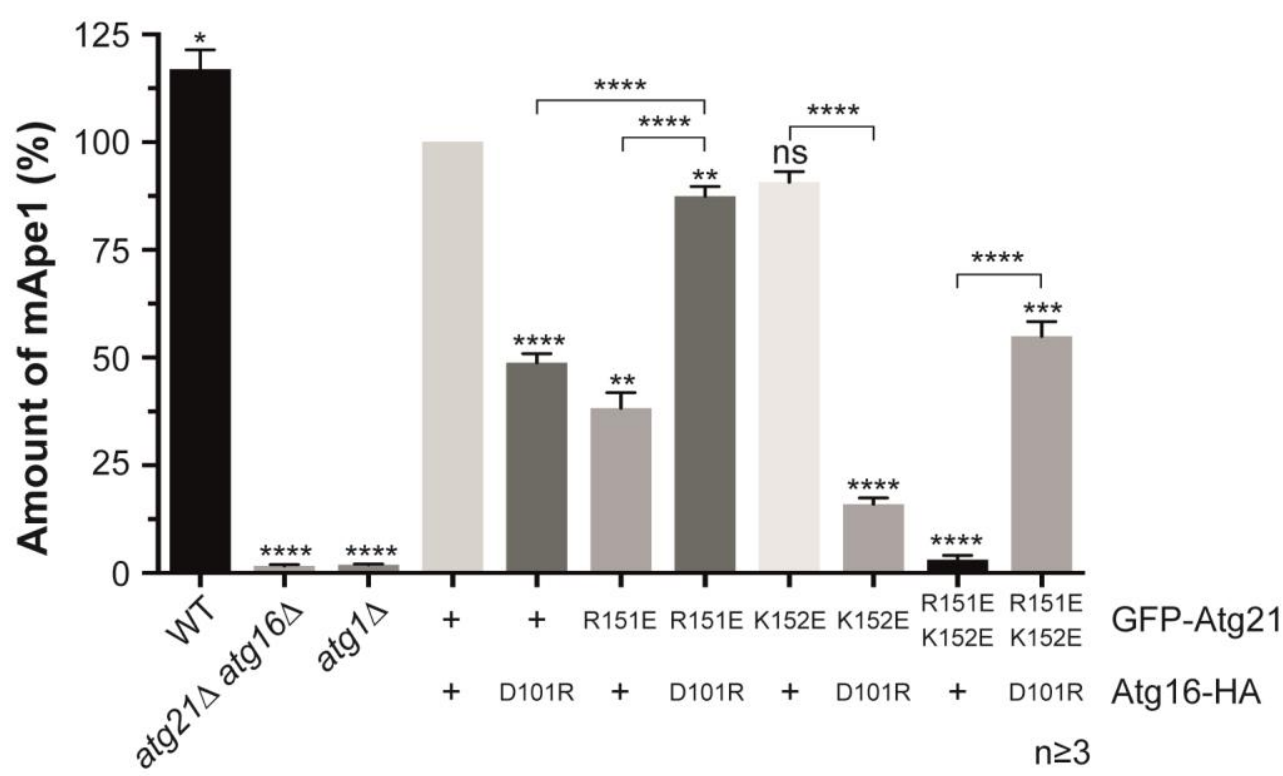

Figure 4.6: Simultaneous charge change of Atg21 R151 (R151E) and Atg16 D101 (D101R) restores the function of the Cvt-pathway

(A) Analysis of the effect of the single and simultaneous mutations of Atg21 and Atg16 on the Cvt-pathway. In an $\operatorname{atg} 21 \Delta \operatorname{atg} 16 \Delta$ strain plasmid-encoded GFP-ATG21 or R151E and K152E single and double mutants were overexpressed in combination with ATG16-HA or the D101R mutant using the MET25 and the CUP1 promoter respectively. Control strains (WT, $\operatorname{atg} 21 \triangle \operatorname{atg} 16 \Delta, \operatorname{atg} 1 \Delta$ ) were transformed with the empty vectors pUG36 and pRS313. Cells were grown over night in selection medium without L-methionine to stationary phase $\left(\mathrm{OD}_{600} 4\right)$, alkaline lysed and analyzed by Western-Blot. The molecular weight marker is depicted in $\mathrm{kDa}$. (B) Quantification of the ratio of mApe1 to the total amount of Ape1 measured in Western-Blot analysis of at least three independent experiments (n). The amount of mApe1 of the strain expressing the WT proteins was set to $100 \%$. 
Statistical relevance was determined using the one sample t-test. Different combinations of WT proteins and mutants were compared using the unpaired two-tailed t-test. Error bars indicate SEM and asterisks indicate p-values: ns, not significant $P>0.05$; ${ }^{*} P<0.05$; ${ }^{* *} P<0.01$; ${ }^{* * *} P<0.001$; **** $P<0.0001$.

Remarkably, the combination of both mutants (Atg16 D101R and Atg21 R151E) restored the pApe1 maturation rate $(87.44 \pm 2.27 \%)$ almost to the level of the WT proteins (Figure 4.6 B). The pApe1 maturation rate was significantly higher than in those strains expressing only one of the mutated proteins. Here, the amount of mApe1 in the cell was reduced to less than $50 \%$ of the WT proteins. The Atg16 D101R mutant also had a positive effect on the Cvt-pathway when combined with the Atg21 R151E K152E double mutant. The pApe1 maturation rate significantly increased from $3.06 \pm 1.04 \%$ in presence of WT Atg16 to $54.91 \pm 3.44 \%$ in presence of the Atg16 D101R mutant. The interaction between Atg21 R151 and Atg16 D101 seemed to be strong enough to even stabilize the Atg21 R151E K152E double mutant to some extent.

When combining the Atg21 K152E mutant with WT Atg16, again the Cvt-pathway was not affected. However, in combination with the D101R mutant of Atg16 a severe block of the Cvt-pathway was observed $(15.98 \pm 1.44 \%$ of the WT proteins) suggesting that there was no interaction between these two residues.

Together with the GFP-TRAP experiments, it became clear that there was a direct interaction, presumably the formation of a salt bridge, between Atg21 R151 and Atg16 D101. Atg21 K152 and Atg16 E102 seemed to only partially contribute to the interaction of both proteins.

Atg21 was reported to be required for the efficient recruitment of Atg16 and therefore also the Atg12 Atg5 conjugate to the PAS (Stromhaug et al., 2004; Juris et al., 2015). Furthermore, the mutation of Atg16 D101A and E102A, that participate in the interaction with Atg21, almost completely abolished PAS recruitment of Atg16 (Juris et al., 2015). Most likely the mutation of Atg21 R151E would also affect the localization of Atg16. This assumption was analyzed by fluorescence microscopy (Figure 4.7). The detection of Atg16 and Atg5 at the PAS as perivacuolar dots in the cell is difficult because both proteins are only transiently located at the PAS. Their PAS pool can be enhanced using mutants defective in the progression of the Cvt-/ autophagic pathway such as an atg84 strain (Stromhaug et al., 2004; Nair et al., 
2010; Juris et al., 2015). Therefore, an $\operatorname{atg} 8 \Delta \operatorname{atg} 21 \Delta \operatorname{atg} 16 \Delta$ strain was used to analyze the localization of Atg16-GFP.
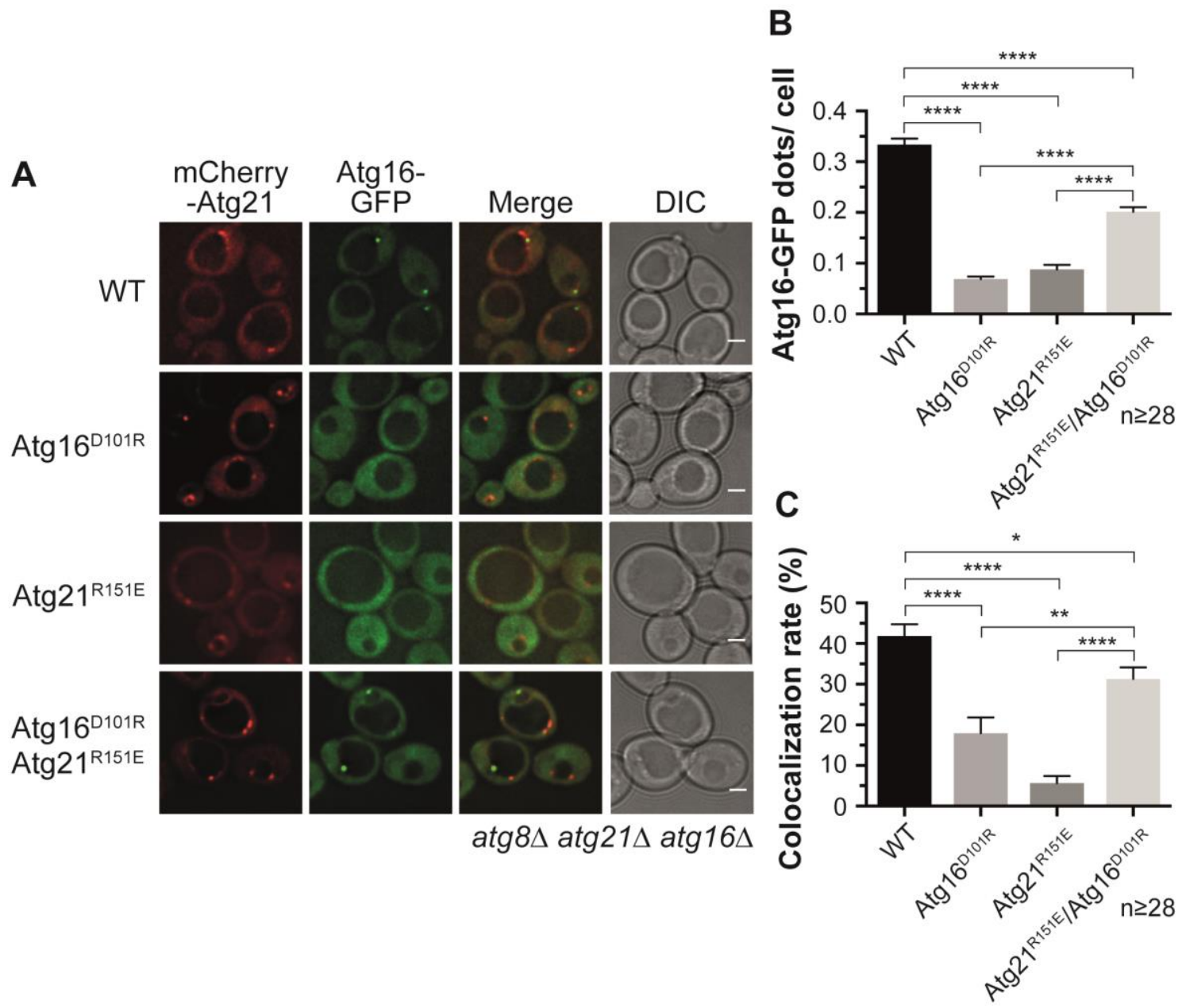

Figure 4.7: Simultaneous charge change of Atg21 R151 (R151E) and Atg16 D101 (D101R) restores the localization of Atg16

(A) Analysis of the localization of Atg16-GFP and mCherry-Atg21 and their mutants in the cell using fluorescence microscopy. In an $\operatorname{atg} 8 \Delta \operatorname{atg} 21 \Delta \operatorname{atg} 16 \Delta$ strain plasmid-encoded mCherry-ATG21 or its R151E mutant were expressed in combination with ATG16-GFP or its D101R mutant using the MET25 and the ATG16 promoter respectively. The cells were grown over night in selection medium with $0.3 \mathrm{mM}$ L-methionine to early stationary phase $\left(\mathrm{OD}_{600} 2\right)$ and subjected to fluorescence microscopy using the DeltaVision microscope equipped with GFP and mCherry filter sets. The number of Atg16-GFP and Atg16 D101R-GFP dots per cell (B) and the number of mCherry-Atg21 or mCherry-Atg21 R151E dots colocalizing with Atg16-GFP dots (C) were counted for each image. In four independent experiments at least 28 images (n) were evaluated resulting in at least 1182 cells counted for each strain. Statistical relevance was determined using the unpaired two-tailed t-test. Error bars indicate SEM and asterisks indicate p-values: ns, not significant $P>0.05$; ${ }^{* * *} P<0.001$; **** $P<0.0001$. Scale bars represent $2 \mu \mathrm{m}$.

The Atg21 R151E and Atg16 D101R mutants were introduced into plasmids expressing mCherry-ATG21 from the MET25 promoter and ATG16-GFP from its endogenous promoter. All possible combinations of WT and mutated plasmids were transformed into the $\operatorname{atg} 8 \Delta \operatorname{atg} 21 \Delta \operatorname{atg} 16 \Delta$ strain. The cells were grown over night in selection medium to early stationary phase $\left(\mathrm{OD}_{600} 2\right)$. The medium was 
supplemented with $0.3 \mathrm{mM}$ L-methionine to induce WT-like expression levels of mCherry-ATG21 and its R151E mutant (Krick et al., 2008a). The cells were visualized using the DeltaVision microscope. The number of Atg16-GFP dots as well as the number of colocalizing mCherry-Atg21 dots were counted (Figure 4.7).

For the WT proteins $0.33 \pm 0.01$ Atg16-GFP dots per cell were observed (Figure 4.7 B). The number of Atg16-GFP dots significantly dropped in cells containing either Atg16 D101R (0.07 \pm 0.005 dots/cell) or Atg21 R151E (0.09 \pm 0.01 dots/cell). This was also true for the colocalization of Atg16-GFP and mCherry-Atg21. While for the WT proteins $41.9 \pm 2.89 \%$ of the Atg16-GFP dots colocalized with mCherryAtg21, the colocalization rate significantly decreased for the respective mutants to less than 20\% (Atg16 D101R-GFP) or even less than 10\% (mCherry-Atg21 R151E) (Figure $4.7 \mathrm{C}$ ). Consistent with the findings of the GFP-TRAP experiments and the effect on the Cvt-pathway, the localization of Atg16-GFP was restored when combining both mutants. Compared to the single mutations, the number of Atg16-GFP dots increased significantly to $0.2 \pm 0.01$ dots per cell. This was also true for the colocalization rate which increased to $31.33 \pm 2.83 \%$.

\subsubsection{Contribution of other amino acids to the interaction of Atg21 and Atg16}

To further characterize the interacting domains of Atg21 and Atg16, the additionally identified amino acids (see chap. 4.1.1.1; Table 4.1, Figure 4.2 yellow arrows and Figure 4.3 yellow residues) were analyzed for their effect on the Cvt-pathway. Therefore, the identified amino acids were mutated into oppositely charged residues (arginines and aspartic acids respectively) except for Atg21 S105 and T106, which were mutated to alanines. As described before, the mutations of Atg16 were introduced into the ATG16-HA and those of Atg21 into the GFP-ATG21 expressing plasmids. In addition, the already existing mutant Atg16 K94A (Juris, 2014) was included into this analysis to evaluate the effect of mutations into uncharged residues in comparison to oppositely charged residues. The mutants were co-expressed with the corresponding ATG16-HA or GFP-ATG21 WT in an $\operatorname{atg} 21 \Delta \operatorname{atg} 16 \Delta$ strain. To evaluate the effect of the single mutants, the ratio of mApe1 to the total amount of Ape1 in the cell was measured in Western-Blot analysis (Figure 4.8). 

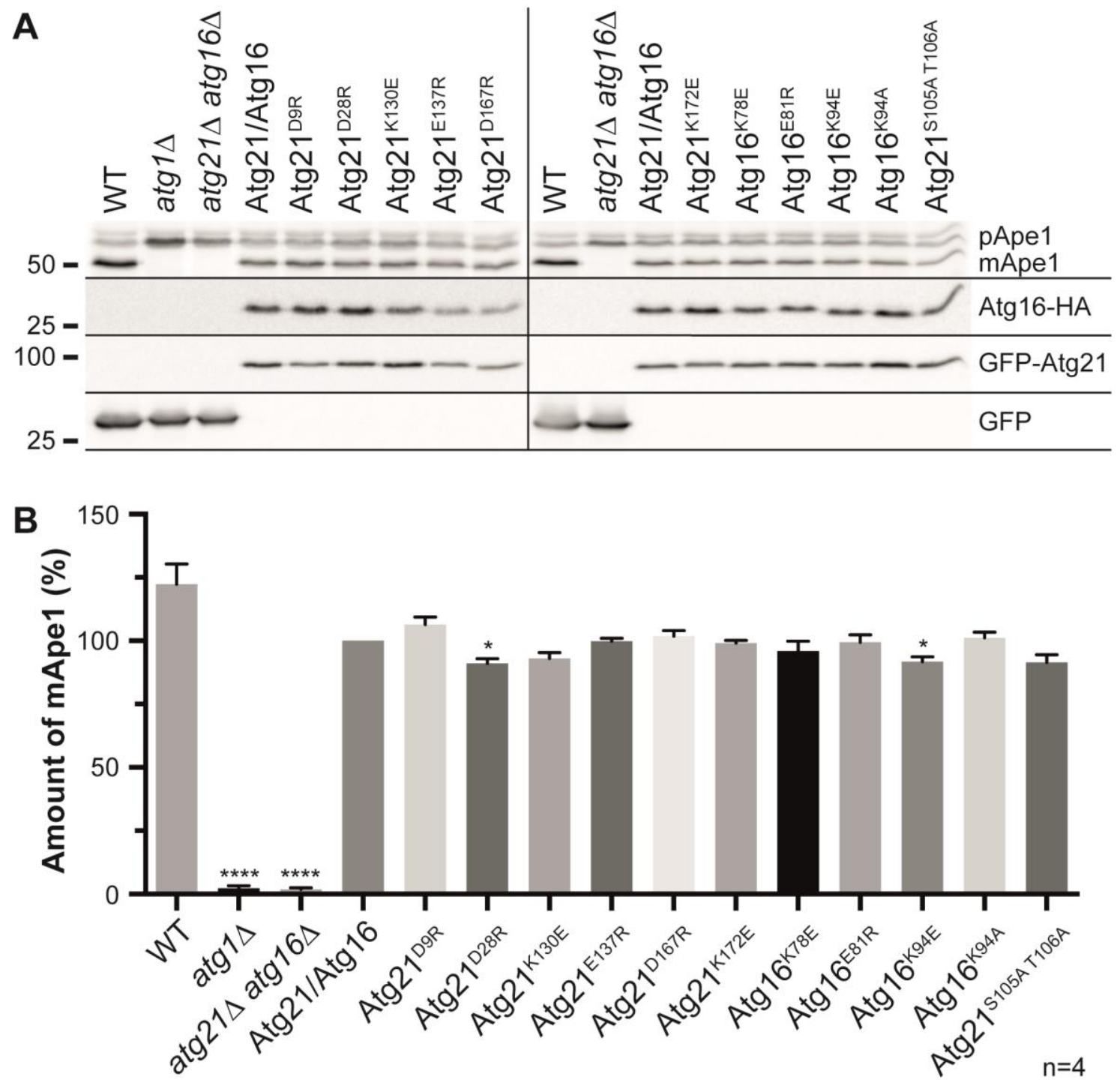

Figure 4.8: Mutation of other potentially interacting amino acids in Atg21 and Atg16 shows no or only minor effects on the Cvt-pathway

(A) Analysis of the effect of the different mutations of Atg21 and Atg16 on the Cvt-pathway measured by the maturation of pApe1. In an atg21 $\operatorname{atg} 16 \Delta$ strain plasmid-encoded GFP-ATG21 or its mutants in combination with ATG16-HA or its mutants were overexpressed using the MET25 and the CUP1 promoter respectively. Control strains (WT, atg14, atg21 $\operatorname{atg} 16 \Delta$ ) were transformed with the empty vectors pUG36 and pRS313. Cells were grown over night in selection medium without L-methionine to early stationary phase $\left(\mathrm{OD}_{600} 2-3\right)$, alkaline lysed and analyzed by Western-Blot. The molecular weight marker is depicted in $\mathrm{kDa}$. (B) Quantification of the ratio of mApe1 to the total amount of Ape1 measured in Western-Blot analysis of four independent experiments (n). The amount of mApe1 of the strain expressing the WT proteins was set to $100 \%$. Statistical relevance was determined using the one sample t-test. Error bars indicate SEM and asterisks indicate $\mathrm{p}$-values: $\mathrm{ns}=$ no asterisk $P$ $>0.05$; $^{*} P<0.05$; ${ }^{* * * *} P<0.0001$.

Remarkably, none of the analyzed mutants showed a severe defect in the maturation of pApe1. Only the mutants Atg21 D28R and Atg16 K94E showed a significant reduction in the pApe1 maturation rate to $90.99 \pm 1.91 \%$ and $91.7 \pm 1.94 \%$, respectively, compared to the WT proteins. The mutants Atg21 K130E and Atg21 S105A T106A also showed a slight reduction in the maturation of pApe1 with 
$93.03 \pm 2.27 \%$ and $91.38 \pm 3.11 \%$ of the WT proteins which however was not significant. In contrast to the Atg16 K94E mutant, the Atg16 K94A mutant did not affect pApe1 maturation rate $(101.2 \pm 2.15 \%$ of the WT proteins) indicating that the net charge on the surface of Atg16 was more important than the K94 itself.

Although none of the residues seemed to severely affect the function of Atg21 and Atg16 in the Cvt-pathway, the mutants Atg21 D28R, Atg21 K130E and Atg16 K94E were selected for further experiments since they showed at least a slight reduction in the pApe 1 maturation rate. Their effect on the interaction of Atg21 and Atg16 as well as the localization of Atg16 was evaluated using the GFP-TRAP approach and fluorescence microscopy.

For the GFP-TRAP approach, GFP-ATG21 and ATG16-HA and their respective mutants were overexpressed from the MET25 and the CUP1 promoter as described before (chap. 4.1.1.2). As a control, the GFP-tag alone was used as a bait to reveal potential unspecific binding of Atg16-HA to GFP.

Interestingly, all three analyzed mutants showed a significant reduction in the interaction of Atg21 and Atg16 (Figure 4.9 A and B). While the Atg21 D28R mutant reduced the amount of bound Atg16-HA to $51.24 \pm 2.57 \%$ compared to the WT proteins, the Atg21 K130E or Atg16 K94E mutant completely abolished the interaction of Atg21 and Atg16 with less than 5\% of bound Atg16-HA or Atg16 K94E-HA. This was comparable to the amount of Atg16-HA isolated in complex with GFP alone ( $9.24 \pm 5.45 \%$ of the WT proteins) and was therefore assumed to be residual unspecific binding.

For fluorescence microscopy, the mutations of Atg21 and Atg16 were introduced into the mCherry-ATG21 and ATG16-GFP expressing plasmids. The mutants were expressed together with the respective WT proteins in an $\operatorname{atg} 8 \Delta \operatorname{atg} 21 \Delta \operatorname{atg} 16 \Delta$ strain as described before (chap. 4.1.1.2). The cells were grown to early stationary phase $\left(\mathrm{OD}_{600} 2\right)$ and analyzed using the DeltaVision microscope (Figure 4.9 C). Again, the number of Atg16-GFP dots per cell and their colocalization with mCherryAtg21 dots was determined (Figure 4.9 D and E). 

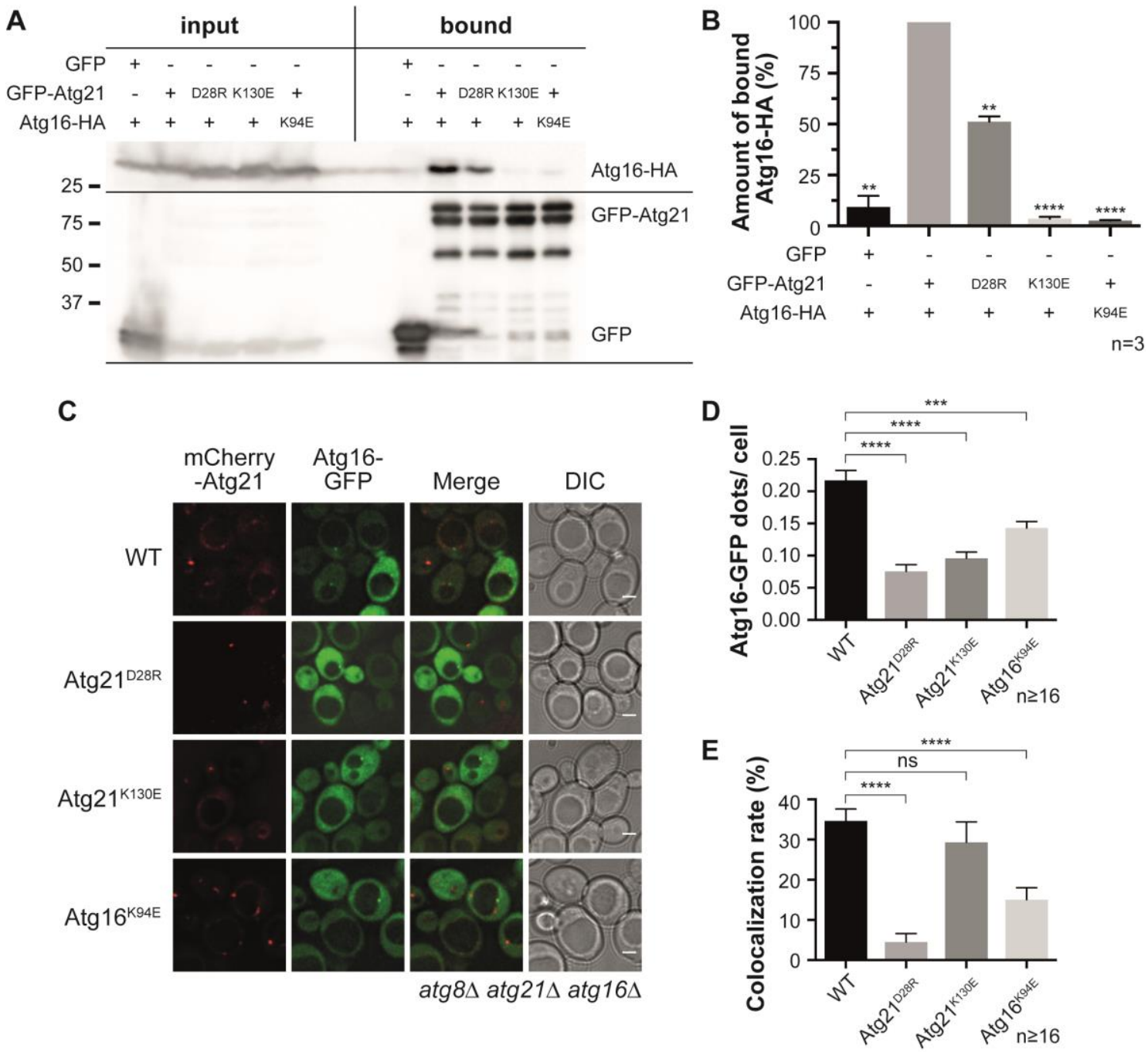

Figure 4.9: Effect of the mutation of Atg21 D28R, Atg21 K130E or Atg16 K94E on the interaction and localization of Atg21 and Atg16

(A) Analysis of the effect of the Atg21 D28R and K130E mutants or the Atg16 K94E mutant on their interaction using the GFP-TRAP approach. In an $\operatorname{atg} 21 \Delta \mathrm{atg} 16 \Delta$ strain the plasmid-encoded bait (GFP, GFP-ATG21 or its D28R and K130E mutants) and the prey (ATG16-HA or its K94E mutant) were overexpressed using the MET25 and CUP1 promoter respectively. Cells were grown in selection medium without L-methionine to early stationary phase $\left(\mathrm{OD}_{600} 2\right)$, osmotically lysed and incubated with GFP-TRAP beads. Input and bound fractions were analyzed by Western-Blot. The molecular weight marker is depicted in $\mathrm{kDa}$. (B) Quantification of three independent GFP-TRAP experiments (n). The amount of bound Atg16-HA or Atg16 K94E-HA was normalized to its amount in the input fraction and the amount of bound GFP-Atg21 or its mutants. The normalized amount of Atg16-HA bound to GFP-Atg21 WT was set to $100 \%$. Statistical relevance was determined using the one sample t-test. (C) Fluorescence microscopy of atg8 $\operatorname{atg} 21 \Delta \operatorname{atg} 16 \Delta$ cells expressing mCherry-ATG21 and ATG16-GFP and their mutants as indicated. The cells were grown over night in selection medium with $0.3 \mathrm{mM}$ L-methionine to early stationary phase $\left(\mathrm{OD}_{600} 2-3\right)$ and subjected to fluorescence microscopy using the DeltaVision microscope. The number of Atg16-GFP and Atg16 K94E-GFP dots per cell (D) and the number of mCherry-Atg21 dots colocalizing with Atg16-GFP dots (E) were counted. In two independent experiments at least 16 images (n) were analyzed resulting in at least 820 cells counted for each strain. Statistical relevance was determined using the unpaired two-tailed t-test. Error bars indicate SEM and asterisks indicate p-values: ns, not significant $P>0.05$; ${ }^{* *} P<0.01$; ${ }^{* * *} P<0.001$; **** $P<0.0001$.

Comparable to the results of the GFP-TRAP, all three mutants significantly reduced the localization of Atg16-GFP to the PAS while increasing its cytosolic localization. 
Whereas for the WT proteins an average of $0.22 \pm 0.02$ Atg16-GFP dots per cell were observed, the number of dots significantly decreased when analyzing the Atg21 D28R (0.08 \pm 0.01$)$, Atg21 K130E (0.1 \pm 0.01$)$ or Atg16 K94E (0.14 \pm 0.01$)$ mutant. Interestingly, the Atg21 D28R mutant showed the greatest effect on the localization of Atg16-GFP while it reduced the interaction with Atg16-HA only to about $50 \%$ of the WT protein in the GFP-TRAP. The mutation of Atg21 D28R and Atg16 K94E also reduced the colocalization of Atg16 and Atg21 while the Atg21 K130E mutant showed no effect. This could be the reason why Atg21 K130E did not significantly reduce the maturation of pApe1 (Figure 4.8).

All together, these findings suggested that the analyzed amino acids do significantly contribute to the net charge on the surface of Atg21 and Atg16, thereby stabilizing the interaction of both proteins. Thus, crucial for the interaction is the formation of a salt bridge between Atg21 R151 and Atg16 D101.

\subsubsection{Dynamics of the formation of the Atg21-Atg16 complex}

Based on the crystal structure of KlAtg21 and the CCD of AgAtg16 in combination with further in vivo and in vitro experiments, the binding mechanism of both proteins could be clarified (chap. 4.1.1). However, the dynamics of Atg21-Atg16 complex formation and dissociation were still elusive. For the formation of the complex, two scenarios can be assumed: Either Atg21 and the Atg12 Atg5/Atg16 complex form a complex in the cytosol that is afterwards targeted to the autophagic membrane by binding of Atg21 to PI3P. Or Atg21 is first targeted to the PAS via PI3P to define the site of lipidation and subsequently recruits the Atg12 Atg5/Atg16 complex as well as the Atg8 Atg3 conjugate.

A variety of approaches exist which can be used to investigate protein-protein interactions. However, none of these methods alone is able to distinguish between a complex that is formed at the membrane or in the cytosol. Therefore, a lipid binding deficient mutant of Atg21 was used in the following experiments. The conserved FRRG-motif of Atg21 mediates its binding to PI3P. Upon mutation of both arginines (R343 and R344) into threonines (Atg21 FTTG) the binding of PI3P is lost and Atg21 is released from the membrane to the cytosol (Stromhaug et al., 2004; Krick et al., 2006). In consequence, the Atg12 Atg5/Atg16 complex is also released 
from the PAS, thereby making the Atg21 $1^{\text {FTTG }}$ mutant a perfect candidate to analyze the interaction of Atg21 and Atg16 in the cytosol. This was investigated, using four different methods, namely the GFP-TRAP approach, fluorescence cross-correlation spectroscopy (FCCS), the split-ubiquitin assay and the BioID assay (chap. 4.1.2.1 to 4.1.2.4). Alongside, the applicability of the used methods to monitor dynamic protein-protein interactions was evaluated.

\subsubsection{Evaluating the interaction of Atg16 and Atg2 ${ }^{\mathrm{FTTG}}$ using the GFP-TRAP approach}

As described in chap. 4.1.1.2, the GFP-TRAP approach was used to evaluate the amount of Atg16-HA isolated in complex with GFP-Atg21 FTTG compared to WT-like GFP-Atg21 (Figure 4.10).
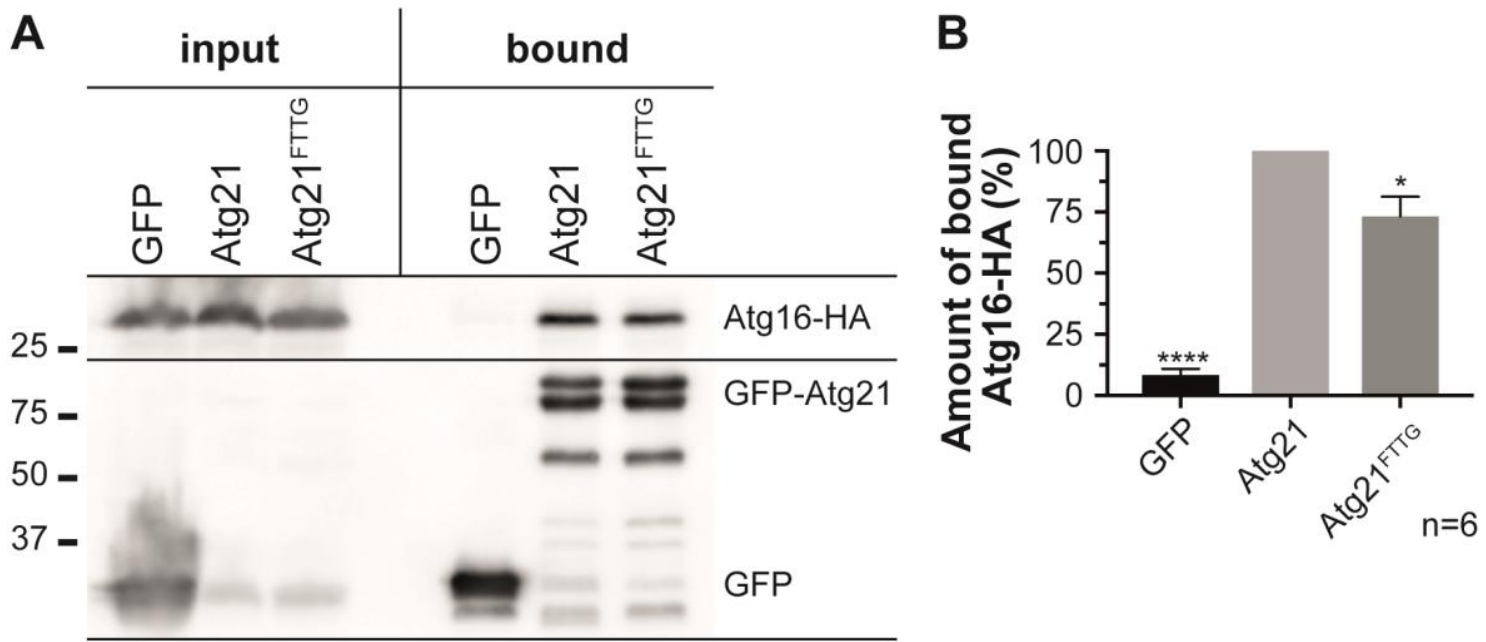

Figure 4.10: Evaluating the interaction of Atg16 and Atg2 $1^{\mathrm{FTTG}}$ using the GFP-TRAP approach

(A) In an $\operatorname{atg} 21 \Delta \operatorname{atg} 16 \Delta$ strain the plasmid-encoded bait (GFP, GFP-ATG21 or GFP-ATG21 ${ }^{F T T G}$ ) and the prey ATG16-HA were overexpressed using the MET25 and CUP1 promoter respectively. Cells were grown in selection medium without L-methionine to early stationary phase $\left(\mathrm{OD}_{600} 2\right)$, osmotically lysed and incubated with GFP-TRAP beads. Input and bound fractions were analyzed by Western-Blot. The molecular weight marker is depicted in $\mathrm{kDa}$. (B) Quantification of six independent GFP-TRAP experiments (n). The amount of bound Atg16-HA was normalized to its amount in the input fraction and the amount of bound GFP or GFP fusion protein. The normalized amount of Atg16HA bound to GFP-Atg21 WT was set to 100\%. Statistical relevance was determined using the one sample t-test. Error bars indicate SEM and asterisks indicate $\mathrm{p}$-values: ${ }^{*} P<0.05$; ${ }^{* * *} P<0.0001$.

In six independent experiments, Atg16-HA was efficiently isolated in complex with GFP-Atg21 and also its FTTG mutant (Figure 4.10 A). This indicated that the mutation of the Atg21 FRRG-motif caused no conformational changes which destroyed the Atg16 binding site. However, quantification of the Western-Blots revealed a significant reduction in the amount of Atg16-HA bound to GFP-Atg21 FTTG (73.24 $\pm 8 \%$ of WT) in comparison to the amount bound to GFP-Atg21 (Figure 
4.10 B). This suggested that there was indeed a difference between the interaction of Atg16 with membrane-bound compared to cytosolic Atg21. However, for the conclusion that the Atg21-Atg16 complex is preferentially formed at the membrane more evidence is necessary. Therefore, additional experiments were performed.

\subsubsection{Analyzing the interaction of Atg16 and Atg21 ${ }^{\mathrm{FTTG}}$ by fluorescence cross-correlation spectroscopy (FCCS)}

FCCS is a spectroscopic method to monitor molecular concentrations, mobilities and interactions by measuring fluorescence fluctuations of fluorophores within a defined observation volume of a sample (Bacia et al., 2006). The diffusion of two molecule species, each fluorescently labeled with one of two spectrally distinct fluorophores (e.g. GFP and mCherry), through the femtoliter-sized observation volume, is recorded as a function of time (Bacia and Schwille, 2003). The obtained intensity traces can be used to calculate autocorrelation curves which provide information on the diffusion coefficient and the local concentration of the fluorescently labeled molecules. The amplitude of the autocorrelation curve negatively correlates with the number of molecules in the observation volume while the decay correlates with the velocity of the moving molecules (González Bardeci et al., 2017).

Interacting molecule species with different labels move together through the observation volume and thereby cause synchronized fluctuations of the fluorescence intensity in both channels. Therefore, besides the autocorrelation curve of each molecule species, a third cross-correlation curve is computed as a readout for the molecular interaction (Bacia and Schwille, 2003). The amplitude of the cross-correlation curve relative to the autocorrelation curves correlates with the number of interacting molecules of both species (González Bardeci et al., 2017). The more molecules interact the higher the cross-correlation, while no interaction results in the absence of cross-correlation (amplitude of zero or 1, depending on the mathematic definition that is used for calculations) (Bacia and Schwille, 2003).

FCCS can be applied to living cells to measure the interaction of fluorescently labeled proteins in the context of their native environment (Bacia et al., 2006) and was therefore used to determine if there was an interaction of Atg16 with Atg21 ${ }^{\text {FTTG in }}$ the cytosol of living yeast cells. As an in vivo control the interaction of Atg16 and 
Atg5 in presence of Atg2 $1^{\text {FTTG }}$ was examined by FCCS, since it is known that the Atg12 Atg5/Atg16 complex forms independent of its association with the autophagic membrane (Kuma et al., 2002).

To be able to record fluorescence fluctuations by FCCS, Atg16 was C-terminally tagged with GFP while the Atg21 $1^{\text {FTTG }}$ mutant and Atg5 were N-terminally tagged with mCherry. In an atg21 $\operatorname{atg} 16 \Delta$ strain plasmid-encoded mCherry- $A T G 21^{\text {FTTG }}$ and ATG16-GFP were expressed using the MET25 and ATG16 promoter respectively. For the control experiment, plasmid-encoded mCherry-ATG5 and ATG16-GFP were expressed together with $A T G 21^{F T T G}$-HA in an $\operatorname{atg} 21 \Delta \operatorname{atg} 16 \Delta \operatorname{atg} 5 \Delta$ strain using the MET25, ATG16 and ATG21 promoter respectively. A prerequisite for the performed FCCS measurements was a low concentration of the fluorescently labeled proteins in the observation volume. Therefore, yeast main cultures were grown in selection medium with a normal amount of L-methionine $(0.78 \mu \mathrm{M})$ to reduce the expression of mCherry-ATG21FTTG and mCherry-ATG5 from the MET25 promoter. The cultures were grown to early stationary phase (OD600 1-2). The FCCS measurements in living yeast cells and following calculations were performed by Dr. Narain Karedla (former member of the III. Institute of Physics, Georg-August-University Göttingen) using alternating-laser excitation (ALEX) with wavelengths $485 \mathrm{~nm}$ and $560 \mathrm{~nm}$ in a custom-made confocal microscope setup (Figure 4.11).

To establish the overlap volume of the two color-foci and to determine the quality of the alignment in the detection channels, FCCS measurements were performed with fluorescent beads which contain dyes emitting in four wavelength channels. The measurements resulted in a cross-correlation amplitude of almost $80 \%$ and served as an in vitro control showing that the method was working (Figure 4.11 A). FCCS was measured with Atg16-GFP and mCherry-Atg21 FTTG in more than 25 cells with 2-3 measurements per cell resulting in a total of 70 collected data points. For all 70 data points, no cross-correlation (amplitude close to 1) was observed suggesting that there was no interaction between Atg16-GFP and mCherry-

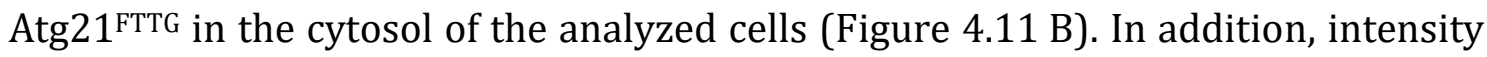
scans of the cells showed an inhomogeneous distribution of both proteins in the cell also pointing to an independent diffusion of both proteins (data not shown). 
In line with published data, a significant cross-correlation was observed in at least 17 evaluable data points of FCCS measurements with Atg16-GFP and mCherry-Atg5 in the presence of Atg21 ${ }^{\text {FTTG}_{-H A}}$ (Figure $4.11 \mathrm{C}$ ). This indicated that there was indeed a formation of the Atg12 Atg5/Atg16 complex in the cytosol of the cells, independent of its association with the autophagic membrane. The ratios of Atg16-GFP or mCherry-Atg5 molecules in complex with each other to free unbound molecules were calculated by the quotient of autocorrelation amplitude and crosscorrelation amplitude (at $\mathrm{t}=0$ ). An average of 32\% of the Atg16-GFP molecules and $17 \%$ of the mCherry-Atg5 molecules were found in complex with each other.

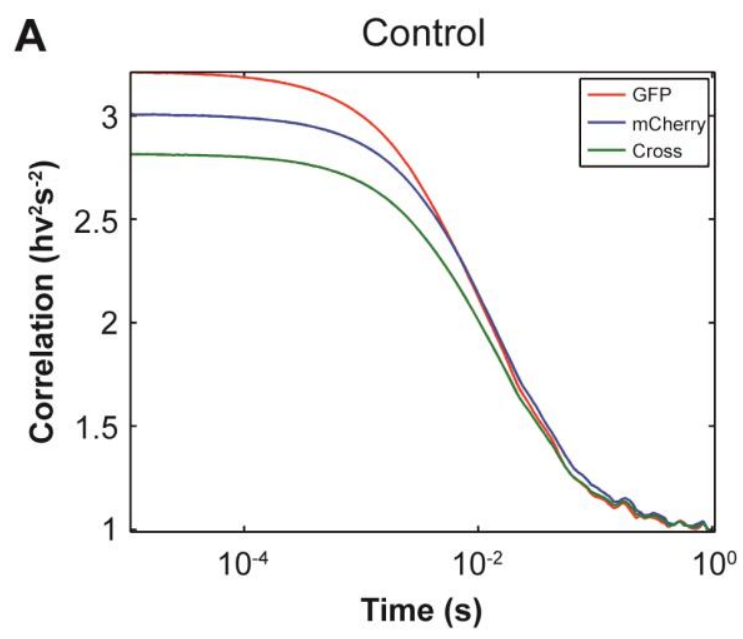

B

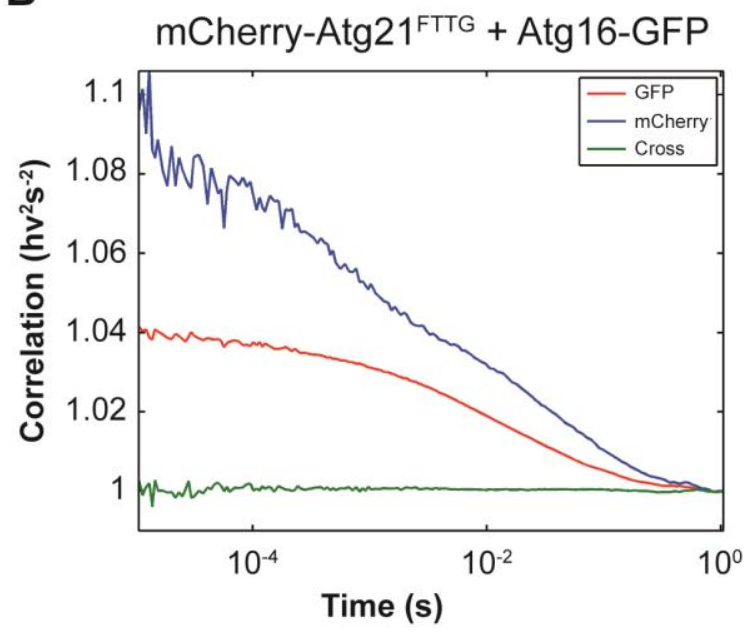

$\operatorname{atg} 21 \Delta \operatorname{atg} 16 \Delta$
C mCherry-Atg5 + Atg16-GFP in presence of Atg2 $1^{\text {FTTG }_{-} \mathrm{HA}}$

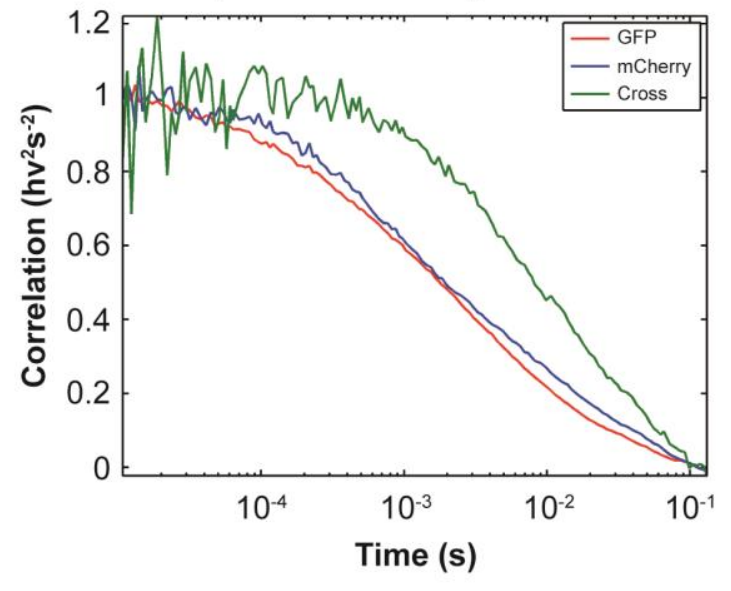

$\operatorname{atg} 21 \Delta \operatorname{atg} 16 \Delta \operatorname{atg} 5 \Delta$

Figure 4.11: In living yeast cells the interaction of Atg16 with Atg21 ${ }^{\text {FTTG }}$ or Atg5 was measured using fluorescence cross-correlation spectroscopy (FCCS)

The FCCS measurements and following calculations were performed by Dr. Narain Karedla (former member of the III. Institute of Physics, Georg-August-University Göttingen) using alternating-laser excitation (ALEX) with wavelengths $485 \mathrm{~nm}$ and $560 \mathrm{~nm}$ in a custom-made confocal microscope setup. (A) FCCS measurement of an in vitro control with fluorescent beads that contain dyes emitting in four wavelength channels. It confirmed that the method was working and was further used to 
establish the overlap volume of the two color-foci as well as to determine the quality of the alignment in the detection channels. (B and C) FCCS measurements of living yeast cells (atg21 $\operatorname{atg} 16 \Delta$ or $\operatorname{atg} 21 \Delta \operatorname{atg} 16 \Delta \operatorname{atg} 5 \Delta$ ) expressing plasmid-encoded ATG16-GFP together with mCherry-ATG21 $1^{\text {FTG }}$ or mCherry-ATG5 and ATG21FTTG-HA using the ATG16, MET25 and ATG21 promoter respectively. Yeast main cultures were grown over night in selection medium $(0.78 \mu \mathrm{M}$ L-methionine) to early stationary phase $\left(\mathrm{OD}_{600}\right.$ 1-2). Autocorrelation curves are shown in red (GFP-fused molecules) and blue (mCherry-fused molecules) and the cross-correlation curve is shown in green. Correlations are plotted with a logarithmic scale.

Together, these findings suggested that the Atg16-Atg21 complex is exclusively formed at the membrane while the Atg12 Atg5/Atg16 complex is assembled in the cytosol. This would support the hypothesis that Atg21 first binds to PI3P at the autophagic membrane before recruiting the Atg12 Atg5/Atg16 complex. However, this only partially corresponded to the results of the GFP-TRAP approach (chap. 4.1.2.1) which revealed residual binding of Atg16 and Atg21 FTTG. Further experiments were performed to clarify this.

\subsubsection{Examining the interaction of Atg16 and Atg21 ${ }^{\mathrm{FTTG}}$ using the split-ubiquitin assay}

The split-ubiquitin assay is a technique similar to the yeast two-hybrid system. It is based on the in vivo reassembly of the $\mathrm{C}$-terminal and the $\mathrm{N}$-terminal ( $\mathrm{C}_{\mathrm{ub}}$ and $\mathrm{N}_{\mathrm{ub}}$ ) half of ubiquitin. Each half is fused to one of the proteins of interest. If these proteins interact, the two parts of ubiquitin are brought into close proximity and are able to form a quasi-native ubiquitin (Johnsson and Varshavsky, 1994).

As a reporter, the Ura3 protein with an additional N-terminal arginine (R-Ura3) is attached to the $\mathrm{C}$-terminus of the $\mathrm{Cub}_{\mathrm{ub}}$ fragment. Ura3, the orotidine 5-phosphate decarboxylase, plays a crucial role in the synthesis of uracil. The expression of URA3 in ura3 defective strains restores their growth on medium lacking uracil. Furthermore, Ura3 converts the nontoxic 5-fluoroorotic acid (5-FOA) into the toxic 5-fluorouracil. Both functions of Ura3 are used as a positive and a negative readout for protein interactions in the split-ubiquitin assay (Wittke et al., 1999).

Upon reconstitution of the quasi-native ubiquitin, it can be recognized by specific ubiquitin proteases which cleave off the R-Ura3 that is attached to the C-terminus of the $\mathrm{Cub}_{\mathrm{ub}}$ fragment. The free R-Ura3 exposes a $\mathrm{N}$-terminal arginine which leads to a rapid degradation of the protein according to the N-end-rule. In consequence, the respective strains show an uracil auxotrophy and a resistance to 5-FOA. When there 
is no interaction between the proteins of interest the strains can grow in medium without uracil and are sensitive to 5-FOA (Wittke et al., 1999).

Using the split-ubiquitin assay, Atg16-Cub was tested for its interaction with $\mathrm{N}_{\mathrm{ub}}$-Atg21, -Atg21 ${ }^{\mathrm{FTTG}}$ and -Atg5 in the SEY $6210 \mathrm{atg} 16 \Delta$ or SEY $6210 \mathrm{atg} 21 \Delta \mathrm{atg} 16 \Delta$ strains as indicated in Figure 4.12. As a positive control for interacting proteins, plasmid-encoded STE14-Cub and $\mathrm{Nub}_{\mathrm{ub}}$ UBC6 were expressed in the SEY $6210 \mathrm{WT}$ strain while STE14-Cub expressed in the presence of an empty vector (pRS314) served as the corresponding negative control. As an additional negative control also ATG16-C $\mathrm{C}_{\mathrm{ub}}$ was expressed in the presence of the empty vector.

For all controls, the expected growth on 5-FOA for interacting proteins (positive control) or without uracil for no interaction (negative control) was observed indicating that the assay was working (Figure 4.12).

In line with the results of the FCCS measurements, Atg16- $\mathrm{Cub}_{\mathrm{ub}}$ was found to interact with $\mathrm{Nub}_{\mathrm{u}} \mathrm{Atg} 5$ in the presence and absence of Atg21, again suggesting that the Atg12 Atg5/Atg16 complex was formed independent of Atg21 and any association with the autophagic membrane.

\begin{tabular}{|c|c|c|c|c|c|c|c|c|c|c|c|}
\hline $\mathbf{C}_{\text {ub }}$ & $\mathbf{N}_{\mathrm{ub}}$ & & $-\operatorname{Tr}$ & $-\mathrm{Hi}$ & & +5 & $=O A$ & & & & Jra \\
\hline Ste14 & Ubc6 & WT & 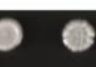 & $\Delta$ & so & e & $y$ & - & & & \\
\hline Ste14 & - & WT & e & 옹 & $=$ & $s$ & & & 0 & $\%$ & ?. \\
\hline Atg16: & Atg21 & $\operatorname{atg} 21 \Delta \operatorname{atg} 16 \Delta$ & e & $\&$ & . & 6 & $\because$ & & ; & $i^{\circ}$ & $\therefore$ \\
\hline Atg16: & $\operatorname{Atg} 21^{\mathrm{FTTG}}$ & $\operatorname{atg} 21 \Delta \operatorname{atg} 16 \Delta$ & 0 & $\%$ & \& & e & $\$$ & : & & & \\
\hline Atg16: & - & $\operatorname{atg} 16 \Delta$ & o & 한 & a & & & & $a$ & 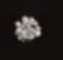 & $=$ \\
\hline Atg16 & Atg5 & $\operatorname{atg} 16 \Delta$ & 0 & $*$ & $s$ & 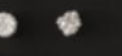 & $s$ & & & & \\
\hline Atg16 & Atg5 & $\operatorname{atg} 21 \Delta \operatorname{atg} 16 \Delta$ & 0 & $\approx$ & 3 & * & $*$ & , & & & \\
\hline
\end{tabular}

Figure 4.12: Examining the interaction of Atg16 with the Atg21 ${ }^{\text {FTTG }}$ mutant using the split-ubiquitin assay

The respective plasmid-encoded $C_{u b}$ and $N_{u b}$ fusions were expressed in the SEY 6210 WT, SEY 6210 $\operatorname{atg} 16 \Delta$ or SEY $6210 \operatorname{atg} 21 \Delta \operatorname{atg} 16 \Delta$ strain as indicated. A serial dilution of 10 -fold dilutions was generated using $1 \mathrm{OD}_{600}$ of yeast precultures and dropped on CM-Trp-His (growth control), CM-TrpHis+5-FOA (growth indicates interaction of tested proteins) and MV-Trp-His-Ura (growth indicates no interaction of tested proteins). Images were taken after three days of growth at $30^{\circ} \mathrm{C}$. Positive control: Ste14-Cub/Nub-Ubc6; negative control: Ste14-Cub/pRS314 (-) and Atg16-Cub/pRS314 (-).

As expected, Atg16-Cub interacted with $\mathrm{Nub}_{\mathrm{ub}}$ Atg21. However, it also showed an interaction with the lipid-binding deficient $\mathrm{N}_{\mathrm{ub}}$-Atg21 $\mathrm{FTTG}$ mutant which 
contradicted the results of the FCCS measurement (chap. 4.1.2.1). According to the observed growth patterns, the interaction of Atg16- $\mathrm{C}_{\mathrm{ub}}$ with $\mathrm{N}_{\mathrm{ub}}-\mathrm{Atg} 21^{\text {FTTG }}$ was even stronger as with the WT-like $\mathrm{Nub}_{\text {-Atg2 }} 1$ suggesting that the cytosolic $\mathrm{Nub}_{\text {ub }}$ Atg21 ${ }^{\text {FTTG }}$ might be better accessible for the interaction with Atg16-Cub. The strong binding of Atg16 and Atg21 $1^{\text {FTTG }}$ disagreed with the results of the GFP-TRAP approach showing a reduced interaction (Figure 4.10). Possibly, the reassembly of the two halves of ubiquitin arrested the complex of Atg16-Cub and $\mathrm{Nub}_{\mathrm{ub}} \mathrm{Atg} 21^{\mathrm{FTTG}}$ and therefore led to an overestimation of the interaction. Due to the inconsistencies in the results of the performed experiments, the BioID assay was used to further evaluate the interaction of Atg16 and Atg21 FTTG.

\subsubsection{Inspecting the interaction of Atg16 and Atg21 ${ }^{\mathrm{FTTG}}$ with the help of the proximity-dependent biotin identification (BiolD) assay}

The BioID assay is another method to investigate protein-protein interactions in their native environment and is frequently used to identify unknown interaction partners of target proteins. It is based on the promiscuous E. coli biotin ligase BirA* which is fused to the protein of interest. Introduced into the cell BirA* will biotinylate surrounding proteins. After cell lysis, these proteins can be affinity purified by streptavidin and analyzed by Western-Blot or MS analysis (for more information see chap. 4.3.1; Roux et al., 2012).

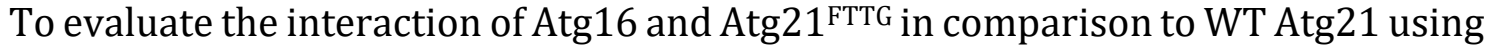
the BioID assay, the genes of ATG16 and ATG21 were cloned into the BirA*-fusion vectors generating BirA*-ATG16, ATG16-BirA* and BirA*-ATG21 fusions (chap. 4.3.1.1 and 3.3.10). The BirA*-fusions were expressed from the MET25 promoter. Corresponding interaction partners were tagged with HA for their detection in Western-Blot analysis. ATG16-HA and ATG21-HA were expressed using the CUP1 and ATG21 promoter respectively. The mutation of the FRRG-motif was introduced into the BirA*-ATG21 and ATG21-HA expressing vectors by site-directed mutagenesis.

BirA* alone and an empty vector (pUG36) served as controls for the unspecific biotinylation of proteins and the background of biotinylated proteins in yeast, respectively. As described for the FCCS measurements (chap. 4.1.2.2), the interaction of Atg16 with Atg5 was analyzed as an additional control. Therefore, 
Atg5 was tagged with two FLAG-tags (Atg5-2xFLAG) under the control of the CUP1 promoter. The respective plasmid combinations were transformed into an $\operatorname{atg} 21 \Delta$ $\operatorname{atg} 16 \Delta$ or an $\operatorname{atg} 21 \Delta \operatorname{atg} 16 \Delta \operatorname{atg} 5 \Delta$ strain as indicated in Figure 4.13 . The strains were grown in selection medium without L-methionine to early stationary phase. Biotinylated proteins were isolated from resulting cell lysates using the $0.2 \mathrm{ml}$ Gravity flow Strep-Tactin ${ }^{\circledR}$ Sepharose ${ }^{\circledR}$ Columns (iba; chap. 3.9.6). Input and elution fractions were analyzed by Western-Blot analysis (Figure 4.13).
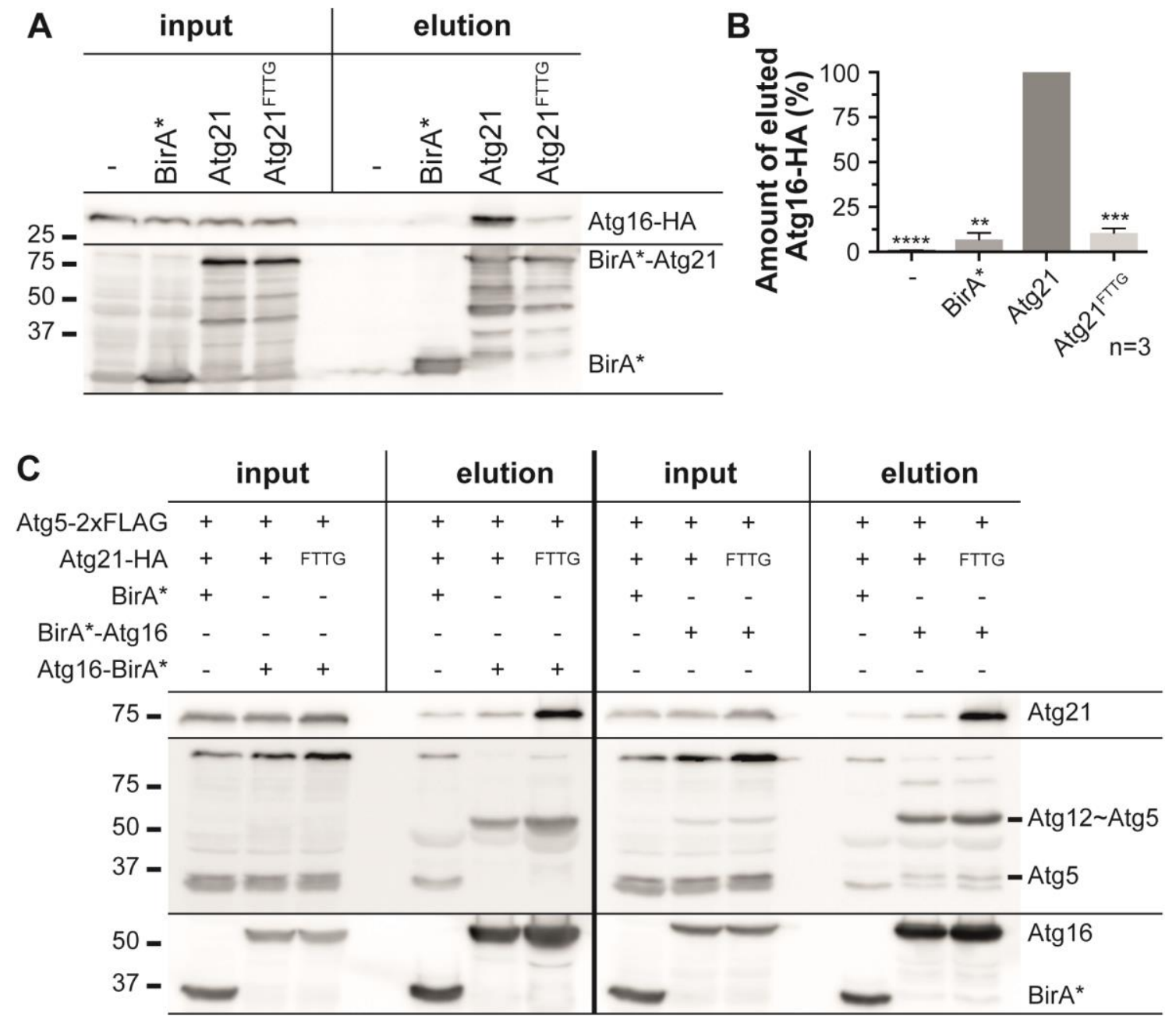

Figure 4.13: Analyzing the interaction of Atg16 and Atg21 ${ }^{\mathrm{FTTG}}$ using the BioID assay

In an $\operatorname{atg} 21 \Delta \operatorname{atg} 16 \Delta$ strain (A) or an $\operatorname{atg} 21 \Delta \operatorname{atg} 16 \Delta \operatorname{atg} 5 \Delta$ strain (C) plasmid-encoded BirA* or its fusion proteins (bait) were expressed together with their HA- or FLAG-tagged interaction partners (prey) as indicated. BirA* alone or the empty vector pUG36 (-) served as controls for unspecific biotinylation or background biotinylation in yeast cells. The bait was expressed using the MET25 promoter while the prey was expressed from the CUP1 promoter or in case of ATG21-HA from its endogenous promoter. Yeast main cultures were grown to early stationary phase $\left(\mathrm{OD}_{600} 2\right)$ in selection medium without L-methionine containing $10 \mu \mathrm{M}$ biotin. Biotinylated proteins were isolated from cell lysates using the $0.2 \mathrm{ml}$ Gravity flow Strep-Tactin ${ }^{\circledR}$ Sepharose ${ }^{\circledR}$ Columns (iba). Input and elution fractions were analyzed by Western-Blot analysis. BirA* and its fusion proteins were detected using anti-Myc antibodies. The molecular weight marker is depicted in $\mathrm{kDa}$. (B) Quantification of three independent BioID experiments (n) using BirA*-Atg21 and BirA*-Atg21 ${ }^{\text {FTTG }}$ as the bait. The amount of eluted Atg16-HA was normalized to its amount in the input fraction. The normalized 
amount of Atg16-HA eluted from the BirA*-ATG21 expressing strain was set to $100 \%$. Statistical relevance was determined using the one sample t-test. Error bars indicate SEM and asterisks indicate p-values: ** $P<0.01$; ${ }^{* * *} P<0.001$; ${ }^{* * * *} P<0.0001$.

As expected, Atg16-HA was found to be efficiently biotinylated and therefore enriched in the elution fraction in the presence of BirA*-Atg21 demonstrating an interaction of both proteins (Figure $4.13 \mathrm{~A}$ and B). The biotinylation of Atg16-HA was absent in the control (cells transformed with empty vector) and significantly reduced in presence of BirA* showing that efficient biotinylation was Atg21dependent. In the presence of the mutant BirA*-Atg21 $1^{\text {FTTG }}$ the level of biotinylated Atg16-HA decreased to $10.52 \pm 2.56 \%$ compared to BirA*-Atg21. The level of biotinylated Atg16-HA was comparable to the observed amount of unspecifically biotinylated Atg16-HA in the presence of BirA* alone (6.86 $\pm 3.64 \%$ of BirA*-Atg21) and suggested that the interaction of Atg16-HA and BirA*-Atg21 FTTG was lost. This highly supported the results obtained from the FCCS measurements and indicated that the Atg21-Atg16 complex was formed at the autophagic membrane and not in the cytosol.

Opposing results were obtained using the $\mathrm{N}$-terminal and C-terminal fusions of Atg16 with BirA* (Figure 4.13 B). Here, Atg21-HA was almost not biotinylated in the presence of BirA*-Atg16 or Atg16-BirA* comparable to the presence of BirA* alone. Only upon mutation of the FRRG-motif in Atg21 FTTG-HA, an efficient biotinylation was observed in the presence of the BirA* fusions of Atg16. This suggested that the interaction of Atg16 with Atg21 was even stronger in the cytosol and therefore supported the observations of the split-ubiquitin assay. The experiments were repeated independently and yielded the same results thereby opposing the results of the FCCS measurements.

The ability of the BirA* fusions of Atg16 to biotinylate interacting proteins was proven by analyzing the biotinylation of Atg5-2xFLAG. Here, Atg5-2xFLAG was found to be biotinylated in the presence of BirA*-Atg16 but not of Atg16-BirA*. The interaction of Atg16 with Atg5 is mediated by its N-terminal domain suggesting that a C-terminal BirA* might be too far away to efficiently biotinylate Atg5-2xFLAG. Independently, the covalently conjugated Atg12 Atg5-2xFLAG complex was efficiently biotinylated by both BirA* fusions of Atg16 indicating that BirA* was functional. The biotinylation of Atg5-2xFLAG or its conjugate with Atg12 was absent 
in the presence of BirA* alone showing that it was Atg16-dependent. Furthermore, the biotinylation was independent of the presence of Atg21-HA or its FTTG mutant supporting the results of the FCCS measurements and the split-ubiquitin assay.

All together the results of the four used methods clearly confirmed the Atg21independent formation of the Atg12 Atg5/Atg16 complex. The formation of the Atg21-Atg16 complex will be detailed in the discussion section (chap. 5.1.3). 


\subsection{Detailed analysis of the localization of autophagy related proteins at the growing phagophore to investigate their role in autophagosome formation}

The second major goal of this study was to understand the temporal and spatial role of Atg21 in the binding and lipidation of Atg8 during autophagosome formation. It is known that after lipidation Atg8-PE carries out two distinct functions. On the inner side of the growing phagophore it interacts with cargo receptors to recruit specific cargos that are subsequently engulfed by the forming autophagosome (Farré and Subramani, 2016). On the outer side Atg8 forms a coat-like structure together with the Atg12 Atg5/Atg16 complex to stabilize the forming autophagosome (Kaufmann et al., 2014). However, the exact location of the lipidation of Atg8 on the growing phagophore is still elusive. To address this question, the precise localization of the components of the lipidation complex, especially of Atg21, at the phagophore was examined.

Due to the small size of the forming phagophore and the resulting autophagosomes (400-900 $\mathrm{nm}$ in diameter), the exact localization of autophagic proteins during autophagosome formation is difficult to determine (Takeshige et al., 1992; Baba et al., 1994). Using fluorescence microscopy, yeast Atg proteins can be observed to localize to a distinct perivacuolar dot defined as the PAS (Suzuki et al., 2001; Suzuki et al., 2007). However, due to current resolution limits of widefield and confocal microscopes (200-250 nm), it cannot be distinguished between the PAS, the elongated phagophore or the already closed autophagosome. To solve this problem, Suzuki et al. (2013) published an assay for living yeast cells that allows a detailed spatial analysis of the localization of Atg proteins at the growing phagophore by normal fluorescence microscopy. They overexpressed APE1 to highly increase the amount of pApe1, the cargo of the Cvt-pathway, in the cell (APE1-overexpression assay). This led to the formation of large spherical structures in the cytosol with an average diameter of $\sim 1.7 \mu \mathrm{m}$ which was at least three times the size of a normal autophagosome and was even visible in bright-field microscopy. GFP-Atg8 formed a cup-like structure around the giant pApe1 complex (GAC) which represented the growing phagophore. Other Atg proteins were either localized to distinct regions of 
the growing phagophore, like the edges or the vacuolar contact site, or covered the whole phagophore (Suzuki et al., 2013).

The APE1-overexpression assay has become a valuable tool and is frequently used for spatial analysis of the growing phagophore. This study, therefore, focused on the establishment of the APE1-overexpression assay to determine the precise localization of Atg21 and the lipidation complex at the growing phagophore. Furthermore, the APE1-overexpression assay was used to investigate the contact site between phagophore and vacuole.

\subsubsection{Establishing and optimizing the APE1-overexpression assay}

Atg8 and Atg16 were repeatedly reported to form cup-like structures around the GAC using the APE1-overexpression assay. Especially Atg8 was used as a marker protein to determine the precise localization of Atg proteins at the growing phagophore (Suzuki et al., 2013; Pfaffenwimmer et al., 2014; Ngu et al., 2015; SakohNakatogawa et al., 2015; Gomez-Sanchez et al., 2018). Therefore, both proteins were suitable candidates for the establishment and the determination of the ideal experimental setup of the APE1-overexpression assay.

To visualize the formation of GACs in the cell by fluorescence microscopy, APE1 was chromosomally tagged with RFP in an $\operatorname{atg} 8 \Delta$ and an atg16 strain. Untagged APE1 was overexpressed from the high copy pYEX-BX vector using the CUP1 promoter (Suzuki et al., 2013). The APE1-carrying pYEX-BX vector was provided by Prof. Dr. Kuninori Suzuki (Graduate School of Frontier Sciences, University of Tokyo). Plasmid-encoded GFP-ATG8 and ATG16-GFP were expressed in the respective strains from their endogenous promoters.

For fluorescence microscopy, the cells were grown over night in selection medium supplemented with $250 \mu \mathrm{M} \mathrm{CuSO}_{4}$ to induce the overexpression of APE1 as described by Suzuki et al. (2013). However, the high amount of copper sulfate in the medium drastically reduced cell growth (data not shown). To reach suitable cell densities, the amount of $\mathrm{CuSO}_{4}$ supplemented to the medium was therefore reduced to $100 \mu \mathrm{M}$ in all following experiments. To find the ideal growth conditions for the formation of the GACs, stationary cells were analyzed by fluorescence microscopy at an $\mathrm{OD}_{600}$ of 2, 4 and 6 . In addition, stationary cells (OD600 5) were transferred to 
SD-N medium, starved for 2 and $4 \mathrm{~h}$ to induce autophagy and also analyzed using the DeltaVision microscope (Figure 4.14). To evaluate the different growth conditions the number of observed GACs and their colocalization with GFP-Atg8 was determined (Figure 4.14 B and C).

A

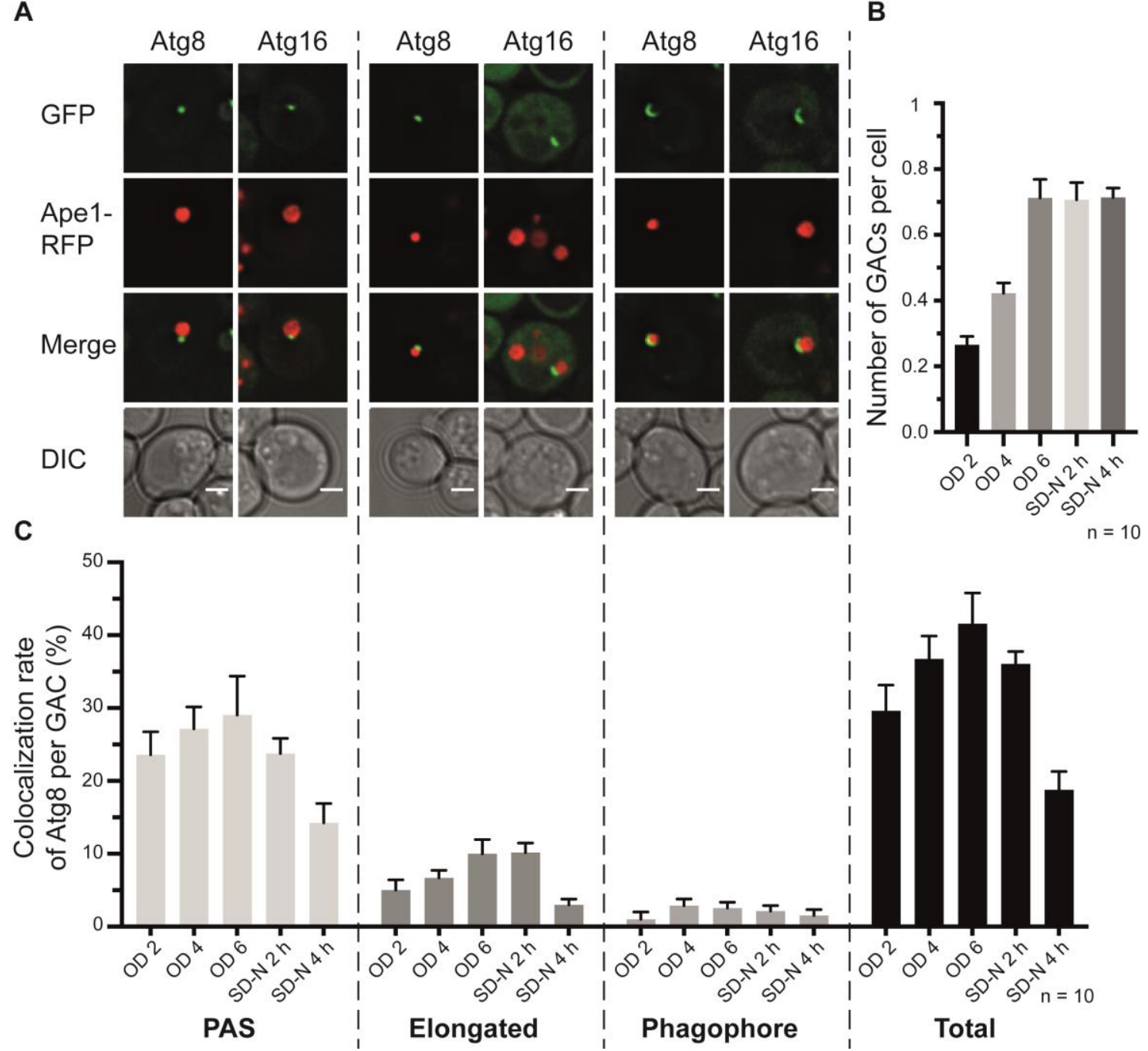

Figure 4.14: Optimization of the experimental setup for the APE1-overexpression assay

(A) Fluorescence microscopy of strains overexpressing APE1 in the presence of Ape1-RFP and GFP-Atg8 or Atg16-GFP as indicated. In an atg8D APE1-RFP or an atg16 APE1-RFP strain APE1 was overexpressed from the high copy pYEX-BX vector using the CUP1 promoter. Plasmid-encoded GFP-ATG8 or ATG16-GFP were expressed using their endogenous promoter. Cells were grown over night in selection medium with $100 \mu \mathrm{M} \mathrm{CuSO}_{4}$. Stationary cells $\left(\mathrm{OD}_{600} 5\right)$ were starved for $2-4 \mathrm{~h}$ in SD-N medium. For fluorescence microscopy, the DeltaVision microscope equipped with GFP and mCherry filter sets was used. Scale bars represent $2 \mu \mathrm{m}$. Under different growing conditions (at an $\mathrm{OD}_{600}$ of 2, 4 and 6 as well as after 2 and $4 \mathrm{~h}$ of starvation (SD-N)), the number of GACs (B) were counted and their colocalization with GFP-Atg8 (C) was determined. The appearance of the colocalizing GFP-Atg8 signal was grouped into dot-like (PAS), slightly elongated and cup-like (Phagophore) structures. The colocalization rate of each group and of the sum of all groups (Total) was calculated. $n$ indicates the number of analyzed images per growing condition resulting in at least 366 counted cells for each condition. 
Within the cells the formation of big red spherical structures was observed proving the formation of GACs. The number of complexes per cell reached their maximum at an $\mathrm{OD}_{600}$ of 6 with an average of 0.7 complexes per cell (Figure $4.14 \mathrm{~B}$ ). After starvation, the number of GACs per cell stayed at its maximum. Furthermore, there was almost no red signal observable in the vacuolar lumen indicating that only very small amounts of pApe1-RFP were transported to the vacuole. As described by Suzuki et al. (2013), the size of the GACs seemed to exceed the cargo capacity of an autophagosome.

Furthermore, the complexes were found to colocalize with the GFP signals of Atg8 and Atg16 (Figure 4.14 A and C). Besides the known perivacuolar dots, also the formation of cup-like structures, engulfing almost half of a GAC, was observed for both proteins. The shape of the GFP signal could be grouped into three different structures: a perivacuolar dot (PAS), a slightly but obviously elongated structure (Elongated) and a cup-like structure (Phagophore). All structures were located between the GAC and the vacuole. The colocalization rate of GFP-Atg8 with the GACs reached its maximum with an average of $42 \%$ (Total) in stationary cells at an $\mathrm{OD}_{600}$ of 6. For cells at an OD 600 of 4 or starved for $2 \mathrm{~h}$ in SD-N also a high colocalization rate of $36 \%$ (total) was observed. The highest colocalization was always observed for cells at an $\mathrm{OD}_{600}$ of 4 to 6 or starved for $2 \mathrm{~h}$. Interestingly, the majority of the GFPAtg8 signals were found to be perivacuolar dots (PAS) colocalizing with 20 to $30 \%$ of the GACs. Only about $1-3 \%$ of the GACs were found to be engulfed by a cup-like structure suggesting that PAS formation was the limiting step. Elongation and breakdown of the phagophore seemed to be much faster.

According to the obtained results of the tested growth conditions, the following experimental setup was used for further APE1-overexpression experiments: If not stated differently, APE1 was overexpressed from the pYEX-BX vector using the CUP1 promoter. Furthermore, fluorescently tagged ATG8 was expressed as a marker for the growing phagophore. Yeast main cultures were grown over night in selection medium supplemented with $100 \mathrm{\mu M} \mathrm{CuSO}_{4}$ to stationary phase with an OD600 up to 4 or 5 to minimize the induction of autophagy due to glucose limitation. Stationary cells were either directly analyzed by fluorescence microscopy or after induction of autophagy in SD-N medium for 1-2 h. 


\subsubsection{Localization of Atg21 at the growing phagophore under different growth conditions}

After optimizing the APE1-overexpression assay, the first aim was to analyze the precise localization of Atg21 at the growing phagophore. Since Atg21 interacts with both, Atg8 and the Atg12 Atg5/Atg16 complex, it could be part of the coat-like structure on the outer side of the phagophore (Kaufmann et al., 2014; Juris et al., 2015). Furthermore, PI3P is reported to be distributed on the outer as well as the inner side of the phagophore but is predominantly enriched on the inner side (Obara et al., 2008a; Cheng et al., 2014). Combining these findings, one could assume that Atg21 is covering the whole phagophore. The APE1-overexpression assay was used to verify this hypothesis.

In a first approach, the $\operatorname{atg} 8 \Delta \operatorname{atg} 21 \Delta$ strain was transformed with the mCherrryATG21, the GFP-ATG8 and the APE1 expressing plasmids. As mCherry-ATG21 was expressed from the MET25 promoter, selection medium containing $0.3 \mathrm{mM}$ L-methionine was used to induce WT-like expression levels of mCherry-ATG21. However, decreased amounts of L-methionine in combination with high amounts of $\mathrm{CuSO}_{4}(100 \mu \mathrm{M})$ in the medium strongly reduced the cell growth while also affecting the fluorescence intensities of GFP-Atg8 and mCherry-Atg21 (data not shown). To avoid additional stress factors for the cells, only endogenous promoters were used for the expression of target genes in further APE1-overexpression experiments.

To elucidate the precise localization of Atg21 at the phagophore in the $\operatorname{atg} 8 \Delta \operatorname{atg} 21 \Delta$ strain, plasmid-encoded ATG21-YFP and mCherry-ATG8 were expressed under the control of their endogenous promoter. In addition, APE1 was overexpressed from the high-copy pYEX-BX vector. The cells were grown as described before (chap. 4.2.1) and analyzed under different growth conditions by fluorescence microscopy. Autophagy was either induced by incubating stationary cells (OD600 4-5) with rapamycin (400 $\mathrm{ng} / \mathrm{ml}$ ) for $1 \mathrm{~h}$ (Figure $4.15 \mathrm{~B}$ ) or by starving them for $1 \mathrm{~h}$ in SD-N medium (Figure 4.15 C and D). In addition, stationary cells were directly subjected to fluorescence microscopy using the DeltaVision microscope (Figure $4.15 \mathrm{~A}$ ).

Interestingly, for both, stationary cells and cells treated with rapamycin, Atg21-YFP showed two distinct localizations at the phagophore. It was either restricted to a 
single dot at the vacuolar contact site of the phagophore or covered the whole mCherry-Atg8 positive phagophore (Figure 4.15 A and B). While the latter observation perfectly matched to the hypothesis that Atg21 was part of the coat-like structure of Atg8 and the Atg12 Atg5/Atg16 complex, the dot-like localization contradicted this assumption and suggested that there might be different functions of Atg21.

A

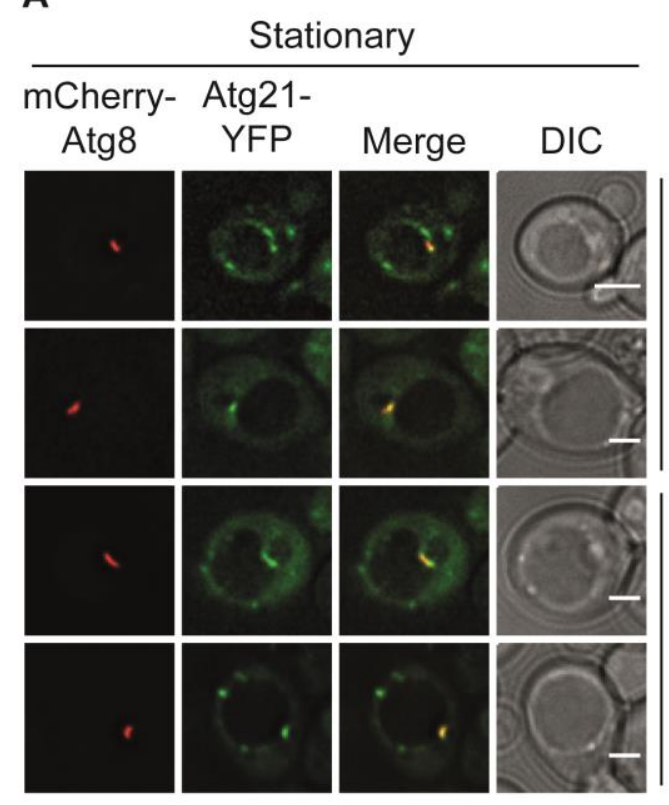

C

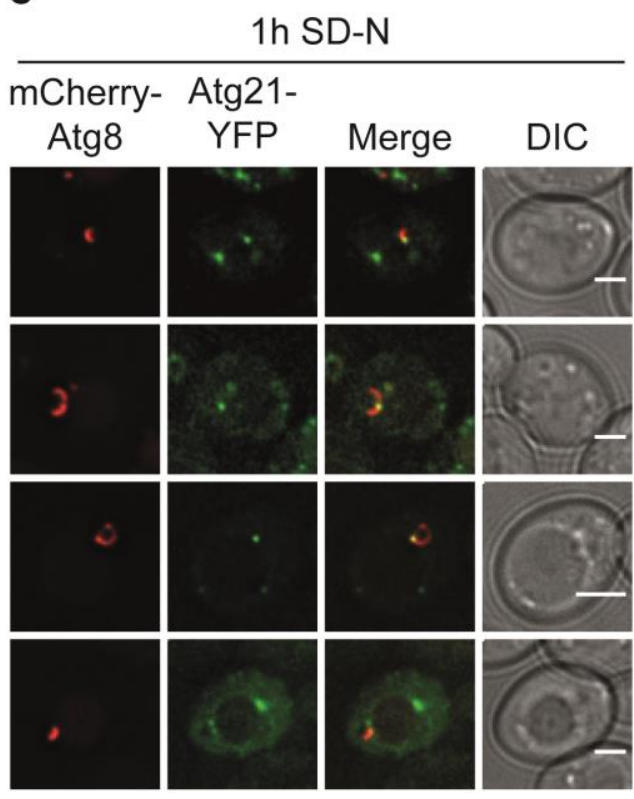

B

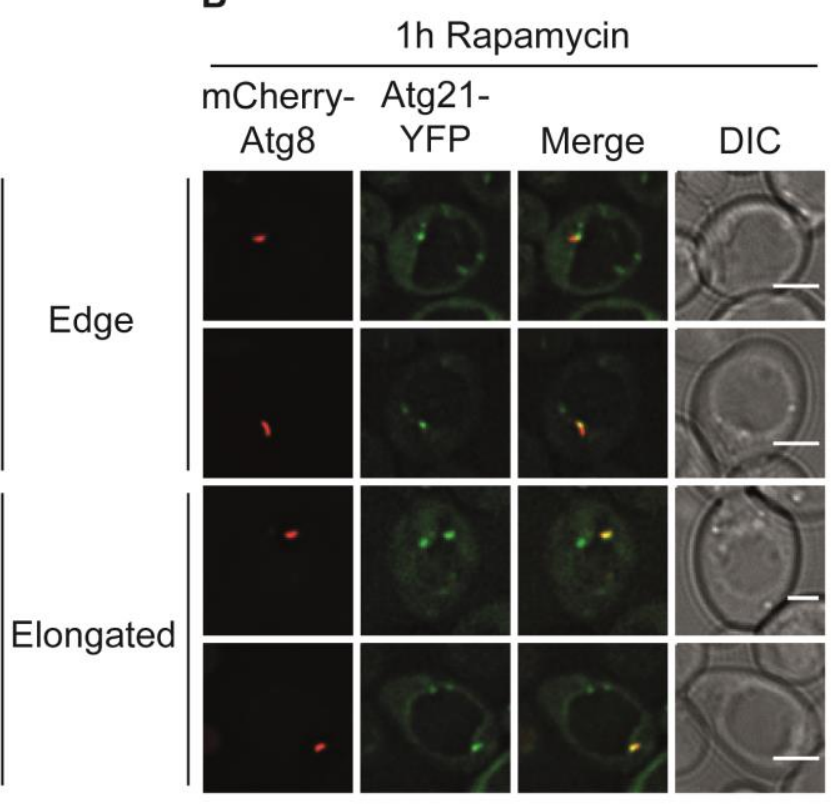

D

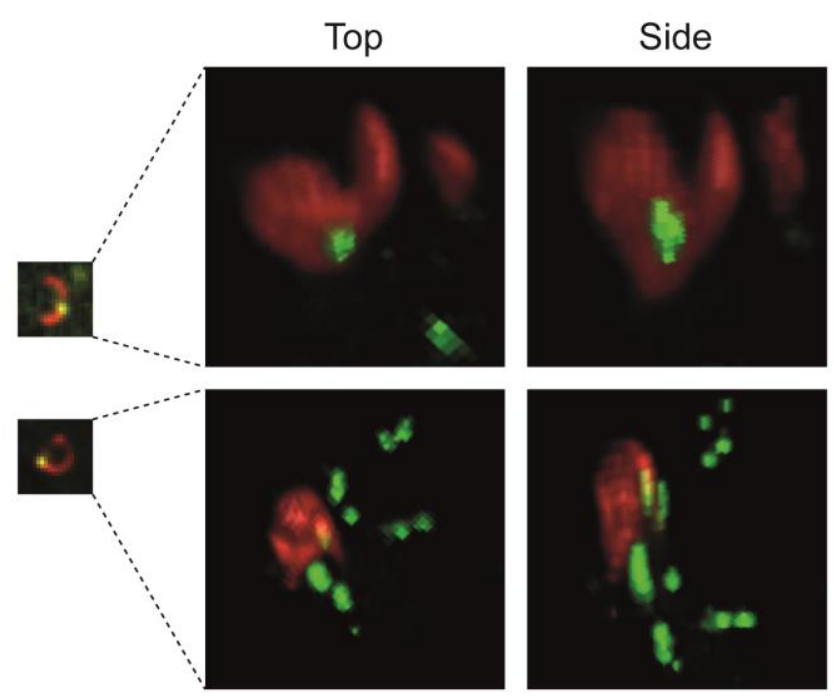

Figure 4.15: Depending on the growth conditions, Atg21-YFP shows different localizations at the mCherry-Atg8 positive phagophore

Analysis of the precise localization of Atg21-YFP at the growing phagophore in APE1-overexpressing cells under different growth conditions. In an atg8 atg21 $\Delta$ strain plasmid-encoded mCherry-ATG8 and ATG21-YFP were expressed using their endogenous promoters. APE1 was overexpressed from 
the high copy pYEX-BX vector using the CUP1 promoter. Cells were grown over night in selection medium with $100 \mu \mathrm{M} \mathrm{CuSO}_{4}$. Stationary cells (OD600 4-5) were either directly analyzed by fluorescence microscopy (A), after incubation with rapamycin $(400 \mathrm{ng} / \mathrm{ml})$ for $1 \mathrm{~h}$ (B) or after starvation in SD-N medium for $1 \mathrm{~h}$ (C and D). For fluorescence microscopy the DeltaVision microscope equipped with GFP and mCherry filter sets was used. Scale bars represent $2 \mu \mathrm{m}$. (D) 3Dprojection of the growing phagophore using the freeware mode of the Huygens Professional X11 software (Scientific Volume Imaging).

Remarkably, under nitrogen starvation Atg21-YFP exclusively localized to a single dot at the contact site between the vacuole and the mCherry-Atg8 positive phagophore (Figure $4.15 \mathrm{C}$ ). This dot was mainly found at that edge of the phagophore directed towards the vacuole. However, in some cases the Atg21-YFP dot also localized to the middle of the phagophore when that part was in contact with the vacuole. Therefore, the contact to the vacuole seemed to predominantly define the position of the Atg21-YFP positive dot under these conditions. Furthermore, the 3D-projection of the taken z-stacks revealed that there was no additional colocalization of Atg21-YFP with the phagophore (Figure 4.15 D).

All together these findings suggested that Atg21 might exhibit different or additional functions depending on the growth conditions and therefore showed a different localization pattern at the phagophore. An additional function of Atg21 in the formation of Cvt-vesicles could explain why Atg21 is essential for the Cvt-pathway but not for unselective bulk autophagy.

\subsubsection{Localization of the Atg8 lipidation complex component Atg3 at the growing phagophore}

It was shown that Atg21 determines the site of Atg8 lipidation by binding PI3P at the autophagic membrane and subsequently recruits Atg8 and the Atg12 Atg5/Atg16 complex (Juris et al., 2015). Together with the observed dot-like localization of Atg21 at the contact site between phagophore and vacuole under nitrogen starvation and therefore autophagy inducing conditions, this would suggest that Atg8 lipidation is restricted to a single site of the phagophore. From that site Atg8-PE would diffuse either to the inner side of the phagophore for cargo recruitment or to the outer side to form the coat-like structure together with the Atg12 Atg5/Atg16 complex. It was already shown that Atg8, Atg16 and Atg5 cover the whole phagophore ((Suzuki et al., 2013); chap. 4.2.1). Since Atg5 is covalently 
conjugated to Atg12 this protein should also cover the whole phagophore. Therefore, the last missing component of the Atg8 lipidation complex was Atg3.

To analyze whether Atg3 is located to a single dot, as observed for Atg21, or covers the whole phagophore, a plasmid was constructed expressing ATG3-GFP from its endogenous promoter. Since N-and C-terminal fusions of GFP to Atg3 were reported to result in non-functional proteins (Suzuki et al., 2007; Ngu et al., 2015), the GFPtag was integrated between D265 and G266 in a disordered region of Atg3 (Ngu et al., 2015). The newly constructed plasmid was transformed together with the mCherry-ATG8 and the APE1-overexpressing plasmids in an $\operatorname{atg} 8 \Delta \operatorname{atg} 3 \Delta$ strain. The cells were grown as described before (chap. 4.2.1) and analyzed by fluorescence microscopy after 2 h starvation in SD-N (Figure 4.16 upper part).

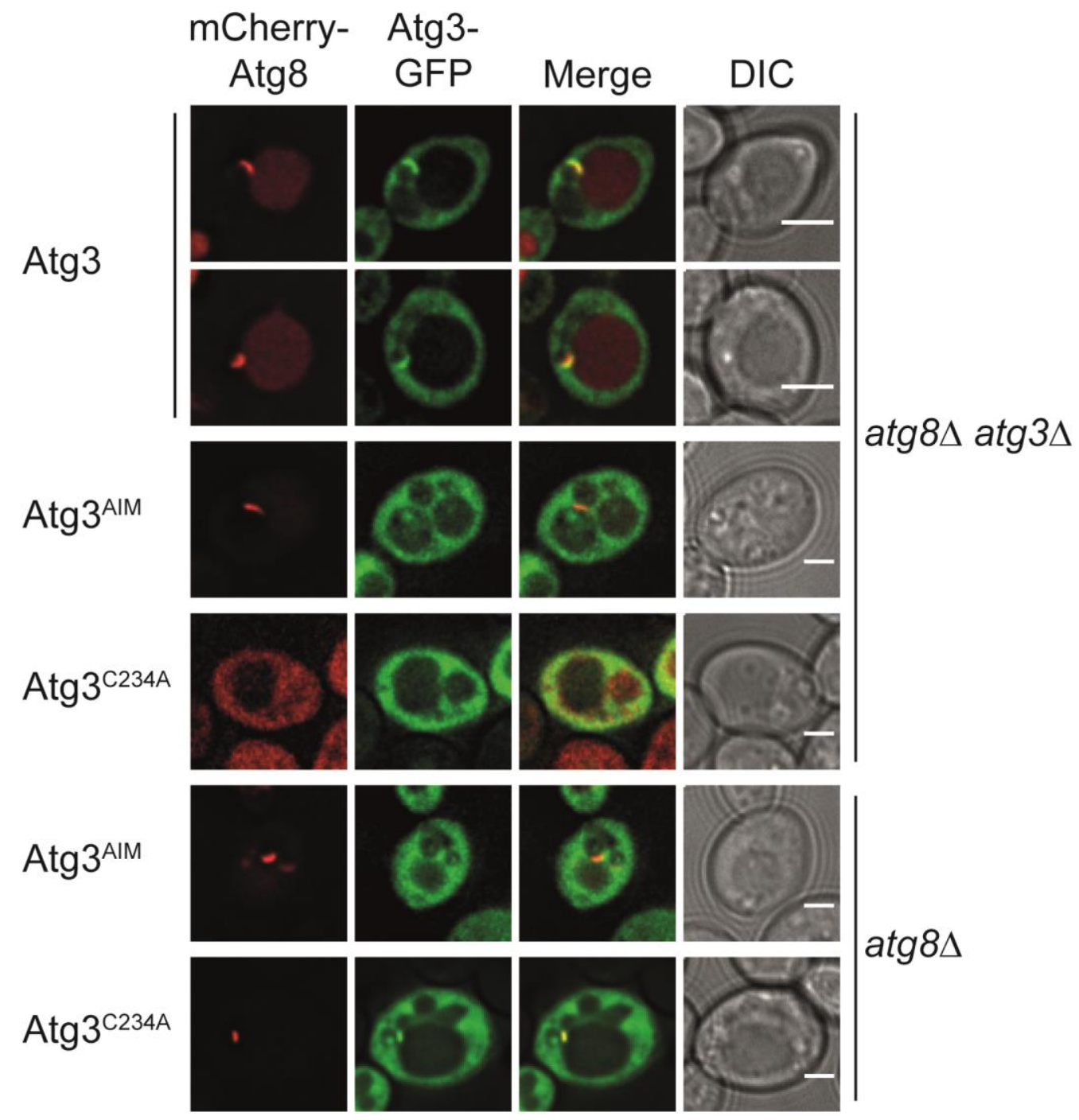

Figure 4.16: Localization of Atg3-GFP and its AIM and C234A mutant at the phagophore

Analysis of the precise localization of Atg3-GFP and its AIM and C234A mutant at the growing phagophore in APE1-overexpressing cells. In an $\operatorname{atg} 8 \Delta \operatorname{atg} 3 \triangle$ or an $\operatorname{atg} 8 \Delta$ strain plasmid-encoded 
mCherry-ATG8 in combination with ATG3-GFP or its AIM and C234A mutant were expressed using their endogenous promoters. APE1 was overexpressed from the high copy pYEX-BX vector using the CUP1 promoter. Cells were grown over night in selection medium with $100 \mu \mathrm{M} \mathrm{CuSO}$. Stationary cells $\left(\mathrm{OD}_{600} 4-5\right)$ were starved for $2 \mathrm{~h}$ in SD-N medium and analyzed by fluorescence microscopy using the DeltaVision microscope equipped with GFP and mCherry filter sets. Scale bars represent $2 \mu \mathrm{m}$.

In contrast to Atg21, Atg3-GFP always covered the whole mCherry-Atg8 positive phagophore and did not form single dots. This perfectly correlated with the reported localization of Atg3 by Ngu et al. (2015) and Sakoh-Nakatogawa et al. (2015). It suggested that either the lipidation of Atg8 is not restricted to a single site of the phagophore or that Atg3 has an additional function at the outer membrane of the phagophore.

For further analysis, two mutations were introduced into the ATG3-GFP expressing plasmid by site-directed mutagenesis. The mutation of both W270A and L273A destroys the AIM of Atg3 and is therefore referred to as the Atg3 AIM mutant. The AIM of Atg3 is not crucial for the lipidation of Atg8 but mediates the high-affinity interaction between both proteins (Yamaguchi et al., 2010). Interestingly, the AIM of Atg3 is essential for the Cvt-pathway but not for starvation-induced autophagy. During Atg8 lipidation, Atg8 is transiently conjugated to a cysteine (C234) of Atg3. Therefore, mutating this cysteine to alanine (C234A) abolishes the conjugase activity of Atg3 (Ichimura et al., 2000). The mutated plasmids were transformed into an $\operatorname{atg} 8 \Delta \operatorname{atg} 3 \Delta$ and an $\operatorname{atg} 8 \Delta$ strain together with the mCherry-ATG8 expressing and the APE1-overexpressing plasmid. The cells were subjected to fluorescence microscopy as described above (Figure 4.16 lower part).

Upon mutation of the Atg3 AIM, its localization at the phagophore was lost while the formation of mCherry-Atg8 positive phagophores was reduced but still detectable. This could also be observed in the presence of endogenous Atg3 in the atg8 8 strain suggesting that the AIM of Atg3 was required for its localization at the phagophore but seemed to be less important for the elongation of the phagophore. In contrast, the Atg3 C234A mutant completely abolished the formation of phagophores and mCherry-Atg8 showed a cytosolic localization. However, in the presence of endogenous Atg3 in the atg84 strain Atg3 C234A-GFP again covered the mCherry-Atg8 positive phagophore probably due to its intact AIM. These findings corresponded to the observations of Sakoh-Nakatogawa et al. (2015). Together this might indicate that the lipidation of Atg8 is restricted to one site of the phagophore. 
Atg3 would then be AIM-dependently distributed over the whole phagophore to fulfill an additional function.

\subsubsection{The distribution of the PI3-kinase complex, PI3P and the PROPPIN Atg18 at the growing phagophore in comparison to Atg21}

The yeast PROPPINs Atg21 and Atg18 bind to membranes via their conserved FRRG-motif (Dove et al., 2004; Stromhaug et al., 2004; Nair et al., 2010). The two arginines of this motif are part of two basic pockets that mediate the binding to PI3P at the autophagic membrane (Krick et al., 2012; Baskaran et al., 2012). PI3P is generated by PI3-kinase complex I which is targeted to the PAS by its unique component Atg14 (Kihara et al., 2001; Obara et al., 2006). Using immuno-electron microscopy, PI3P was observed to reside on the inner and outer membrane of the phagophore but is predominantly enriched on the inner membrane in yeast (Obara et al., 2008a; Cheng et al., 2014). In contrast, Atg21 was observed to be restricted to one site of the phagophore (chap. 4.2.2) suggesting that there need to be additional mechanisms, apart from PI3P-binding, which determine the localization of Atg21. To gain further insights into the interdependence of the distribution of PI3P, the PI3kinase complex and the PROPPINs at the phagophore, their precise localization was analyzed using the APE1-overexpression assay.

Atg14 was chosen as a representative of the PI3-kinase complex I. For visualization by fluorescence microscopy, it was chromosomally tagged with three GFP molecules (3xGFP). The 3xGFP-tag was used since a single GFP tagged to Atg14 was not bright enough (personal communication of Dr. Roswitha Krick). The 3xGFP-tag was introduced at the C-terminus of Atg14 in a WT and an atg8 strain. The expression and biological activity of ATG14-3xGFP was confirmed by pApe1 maturation (data not shown). In the $\operatorname{atg} 8 \triangle A T G 14-3 x G F P$ strain, plasmid-encoded mCherry-ATG8 and $A P E 1$ were expressed as described in former experiments (chap. 4.2.1 and 4.2.2). PI3P was visualized using the PI3P-binding 2xFYVE-domain of mammalian Hrs (hepatocyte growth factor-regulated tyrosine kinase substrate) which was tagged to mRFP (Obara et al., 2008a). In an atg8D strain mRFP-FYVE was expressed together with plasmid-encoded GFP-ATG8 and APE1 using the TEF, the ATG8 and the CUP1 promoters respectively. Both strains were grown as described before (chap. 
4.2.1) and either analyzed directly by fluorescence microscopy or after 1-2 $\mathrm{h}$ starvation in SD-N medium (Figure 4.17 A and C).

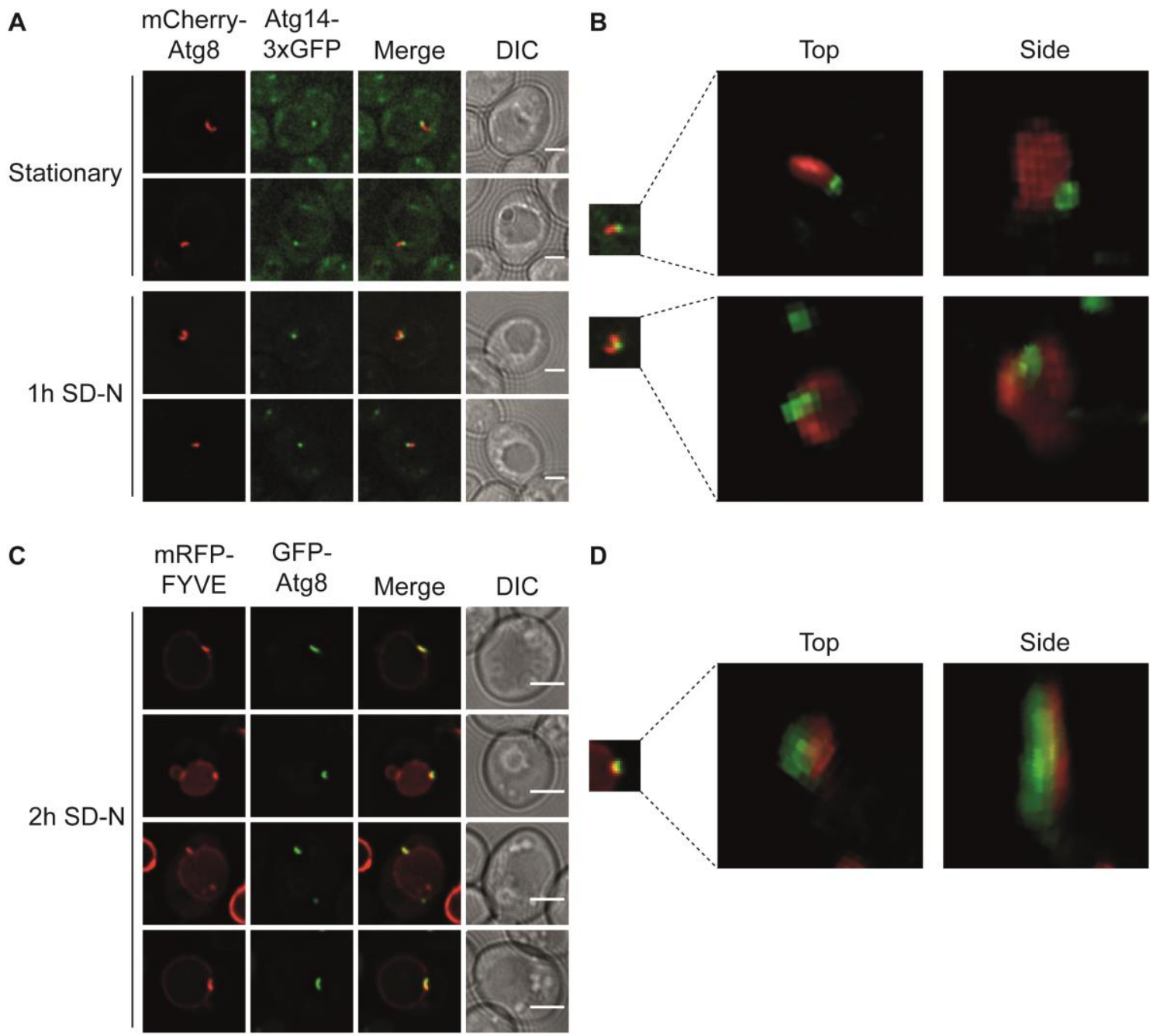

Figure 4.17: Atg14 localizes to a dot at the contact site of vacuole and phagophore while PI3P covers the whole phagophore

Analysis of the precise localization of Atg14-3xGFP (A and B) and PI3P (C and D) at the growing phagophore in APE1-overexpressing cells. (A and B) For visualization of Atg14 the atg8D ATG14$3 x G F P$ strain was used. (C and D) PI3P was visualized in an atg8 strain by expressing plasmid encoded mRFP-2xFYVE(Hrs) using the TEF promoter. In both strains plasmid-encoded mCherryATG8 and APE1 were expressed using the ATG8 and CUP1 promoter respectively. Cells were grown over night in selection medium with $100 \mu \mathrm{M} \mathrm{CuSO}$. Stationary cells $\left(\mathrm{OD}_{600} 4-5\right)$ were analyzed directly or after 1-2 $\mathrm{h}$ starvation in SD-N medium by fluorescence microscopy using the DeltaVision microscope equipped with GFP and mCherry filter sets. Scale bars represent $2 \mu \mathrm{m}$. (B and D) 3Dprojections of the growing phagophore using the freeware mode of the Huygens Professional X11 software (Scientific Volume Imaging).

Atg14-3xGFP localized to a single dot at the vacuolar contact site of the mCherry-Atg8 positive phagophore similar to the dot observed with Atg21-YFP (Figure 4.17 A). In contrast to Atg21, there were no discrepancies observed between the localization of Atg14-3xGFP in stationary phase and under nitrogen starvation (1 h SD-N). 3D-projections of the growing phagophores also revealed no additional 
colocalization of Atg14-3xGFP with the elongating phagophore (Figure 4.17 B). This perfectly correlated with the reported localization of Atg14 by Suzuki et al. (2013). As expected, mRFP-FYVE localized to the vacuolar membrane, some perivacuolar punctate structures, assumed to be endosomes, and to the phagophore (Figure $4.17 \mathrm{C}$ ). It perfectly colocalized with GFP-Atg8 and therefore covered the whole phagophore. This was also confirmed by the 3D-projections of the growing phagophore and correlated with the reported localization of the FYVE-domain and PI3P (Obara et al., 2008a; Cheng et al., 2014). Based on fluorescence microscopy it was not possible to distinguish whether mRFP-FYVE was bound to the inner or the outer side of the phagophore.

To analyze the localization of Atg18, a new plasmid was constructed carrying ATG18-GFP under the control of its endogenous promoter. It was expressed in an $\operatorname{atg} 8 \Delta \operatorname{atg} 18 \Delta$ strain together with mCherry-ATG8 and APE1 as described before (chap. 4.2.1 and 4.2.2). Stationary cells were directly analyzed by fluorescence microscopy or after 1-3 h starvation in SD-N (Figure 4.18).

In contrast to Atg21, Atg18-GFP localized to both edges of the mCherry-Atg8 positive phagophore for all analyzed growth conditions. However, the 3D-projection of the growing phagophores revealed that Atg18-GFP did not localize into single dot-like structures but instead was covering parts of the rim of the growing phagophore (Figure 4.18 B). This became most obvious in Figure 4.18 C and D. Here, the imaged mCherry-Atg8 positive phagophore seemed to already engulf at least half of the GAC and Atg18-GFP localized directly on top of its rim. However, Atg18 did not always cover the whole rim of the phagophore. The observed localization of Atg18-GFP correlates with the reported localization of Atg18 and its interaction partner Atg2 (Suzuki et al., 2013; Gomez-Sanchez et al., 2018).

Under nitrogen starvation conditions, Atg21 and Atg18 showed two distinct localizations at the phagophore which both clearly differed from the observed distribution of PI3P. All together these findings suggested the necessity of additional mechanisms, apart from PI3P-binding, that restrict the localization of both, Atg21 and Atg18, to the observed sites. 
A

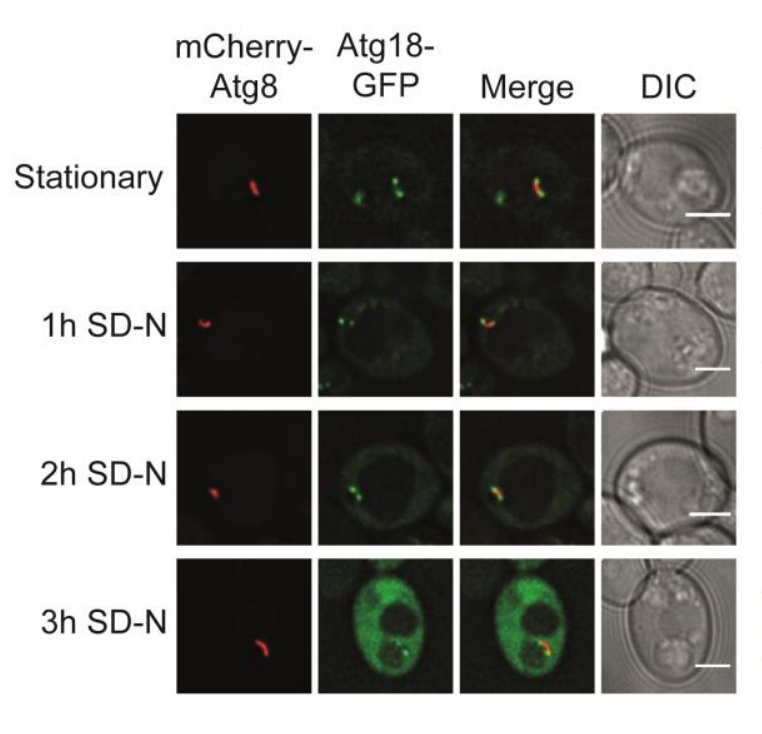

C

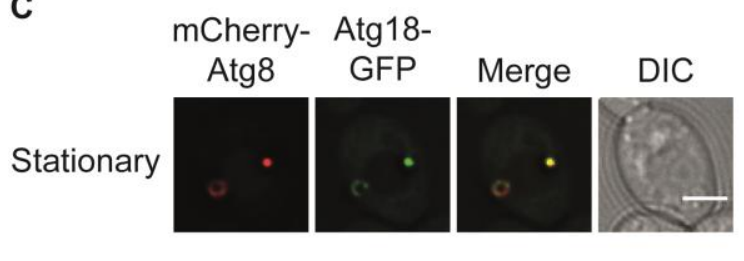

B
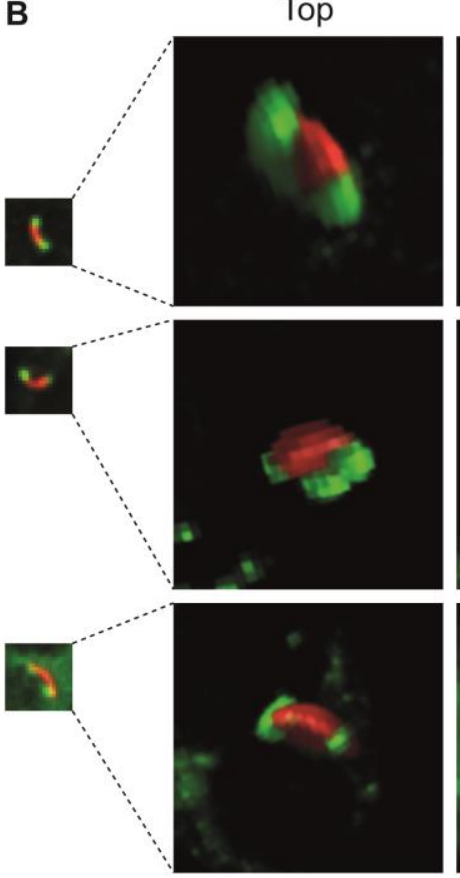

D

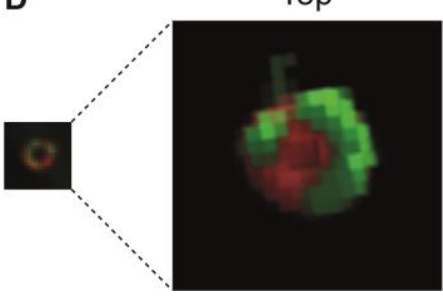

Side
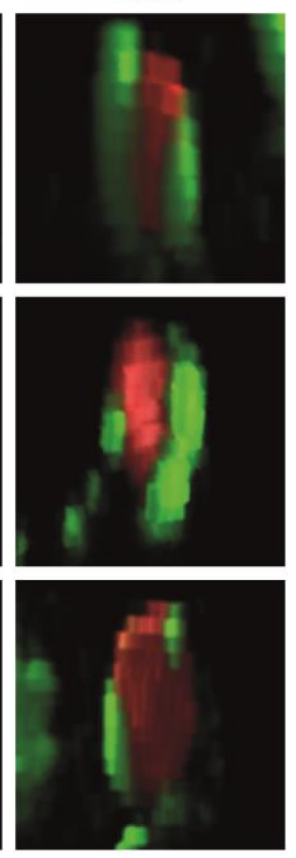

Side

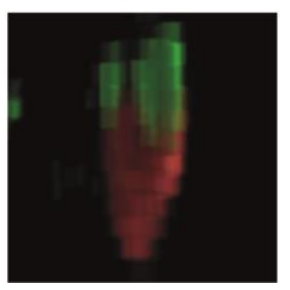

Figure 4.18: Atg18 is restricted to the rim of the growing phagophore

Analysis of the precise localization of Atg18-GFP (A and B) at the growing phagophore in APE1-

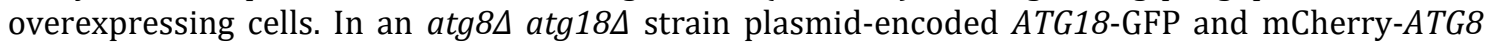
were expressed using their endogenous promoters. APE1 was overexpressed from the high copy pYEX-BX vector using the CUP1 promoter. Cells were grown over night in selection medium with $100 \mu \mathrm{M} \mathrm{CuSO}$. Stationary cells $\left(\mathrm{OD}_{600} 4-5\right)$ were directly analyzed or after starvation for $1-3 \mathrm{~h}$ in SD-N medium by fluorescence microscopy using the DeltaVision microscope equipped with GFP and mCherry filter sets (A and C). Scale bars represent $2 \mu \mathrm{m}$. (B and D) 3D-projections of growing phagophores using the freeware mode of the Huygens Professional X11 software (Scientific Volume Imaging). 


\subsubsection{The contact site of the phagophore and the vacuolar membrane and the role of Vac8 in phagophore formation}

In all performed experiments, the formation of the phagophore was always observed in close proximity to the vacuolar membrane. This indicated that there might be a direct contact between the vacuole and the phagophore. For the formation and maintenance of such a contact site, an interaction between proteins at the phagophore and the vacuolar membrane would be required. These proteins could also be interaction partners of Atg21 and could thereby restrict its localization to the contact site between phagophore and vacuole. It could be even possible that Atg21 itself contributed to this contact site.

The vacuolar membrane protein Vac8 is an armadillo-repeat protein consisting of 12 armadillo repeats (Wang et al., 1998; Pan and Goldfarb, 1998; Jeong et al., 2017). Armadillo-repeat proteins serve as protein-binding platforms and are functionally very versatile (Tewari et al., 2010). This becomes obvious looking at the multiple functions of Vac8. It is a key player in vacuole inheritance and regulates homotypic vacuole fusion (Wang et al., 1998; Pan and Goldfarb, 1998; Wang et al., 2001). Furthermore, it participates in the formation of the nucleus-vacuole junction (NVJ), the contact site between perinuclear and vacuolar membranes, by directly interacting with Nvj1 (Pan et al., 2000). Interestingly, it has a crucial role in the Cvt-pathway through the interaction with Atg13 (Wang et al., 1998; Scott et al., 2000). Vac8 is both myristoylated and palmitoylated which targets it to the vacuolar membrane (Wang et al., 1998; Subramanian et al., 2006).

Since Vac8 is a vacuolar membrane protein that participates in the Cvt-pathway and regulates membrane contact sites, it was the ideal candidate to also regulate the contact between the vacuolar and the autophagic membrane. Therefore, its role in the formation of the phagophore was further analyzed (chap. 4.2.5.1, 4.2.5.2 and 4.2.5.4).

\subsubsection{Vac8 is enriched at the contact site between vacuole and phagophore}

Due to its myristoylation and palmitoylation, Vac8 is targeted to the vacuolar membrane. However, it is not evenly distributed but enriched at those parts of the vacuolar membrane that form the NVJ (Pan et al., 2000). If Vac8 also participates in 
a contact between vacuolar membrane and phagophore, it would be possibly similarly enriched there. To prove this assumption, the distribution of Vac8 at the vacuolar membrane in relation to the phagophore was analyzed using the APE1overexpression assay.

To visualize Vac8 by fluorescence microscopy, it was either C-terminally tagged with GFP on a plasmid or chromosomally with 3xGFP. The expression and biological activity of $V A C 8$-GFP and $V A C 8-3 \times G F P$ was verified by pApe1 maturation (data not shown). Vac8-3xGFP was not fully active, nevertheless both strains, the atg $8 \Delta$ vac8 $\Delta$ strain expressing plasmid-encoded VAC8-GFP using its endogenous promoter and the atg8D VAC8-3xGFP strain, were further analyzed. In both strains, plasmidencoded mCherry-ATG8 and APE1 were expressed as described before (chap. 4.2.1 and 4.2.2). Stationary cells were either analyzed directly by fluorescence microscopy or after starvation for 1-2 $\mathrm{h}$ in SD-N medium (Figure 4.19).

As expected, Vac8-GFP was not evenly distributed at the vacuolar membrane. Usually, it was enriched in one bigger raft, probably representing the NVJ, but it also formed some additional dot-like structures at the vacuolar membrane. Some of these dot-like structures often colocalized with the mCherry-Atg8 positive phagophore. The measured fluorescence profiles at these putative vacuolephagophore contact sites confirmed that there was a colocalization of GFP and mCherry fluorescence maxima (Figure 4.19). The accumulation of Vac8-GFP into dot-like structures and their colocalization with the phagophore was observed under all analyzed growth conditions and became even more obvious when looking at 2D- and 3D-projections of the taken z-stacks of the analyzed cells (Figure 4.19 B and D). The 2D- and 3D-projections also revealed that there was no connection of the mCherry-Atg8 positive phagophore or the dot-like structures of Vac8-GFP with the bigger rafts of Vac8-GFP which likely represent NVJs.

However, for some of the analyzed cells no enrichment of Vac8 was observed in proximity to the forming phagophore (data not shown). Most of those cells showed a very bright signal of Vac8-GFP all over the vacuolar membrane which was probably too intense to distinguish enriched structures. However, this could also mean that a Vac8-dependent connection between the phagophore and the vacuole 
was not always formed. The differences in the fluorescence intensity of Vac8-GFP could be caused by differing expression levels due to plasmid expression.

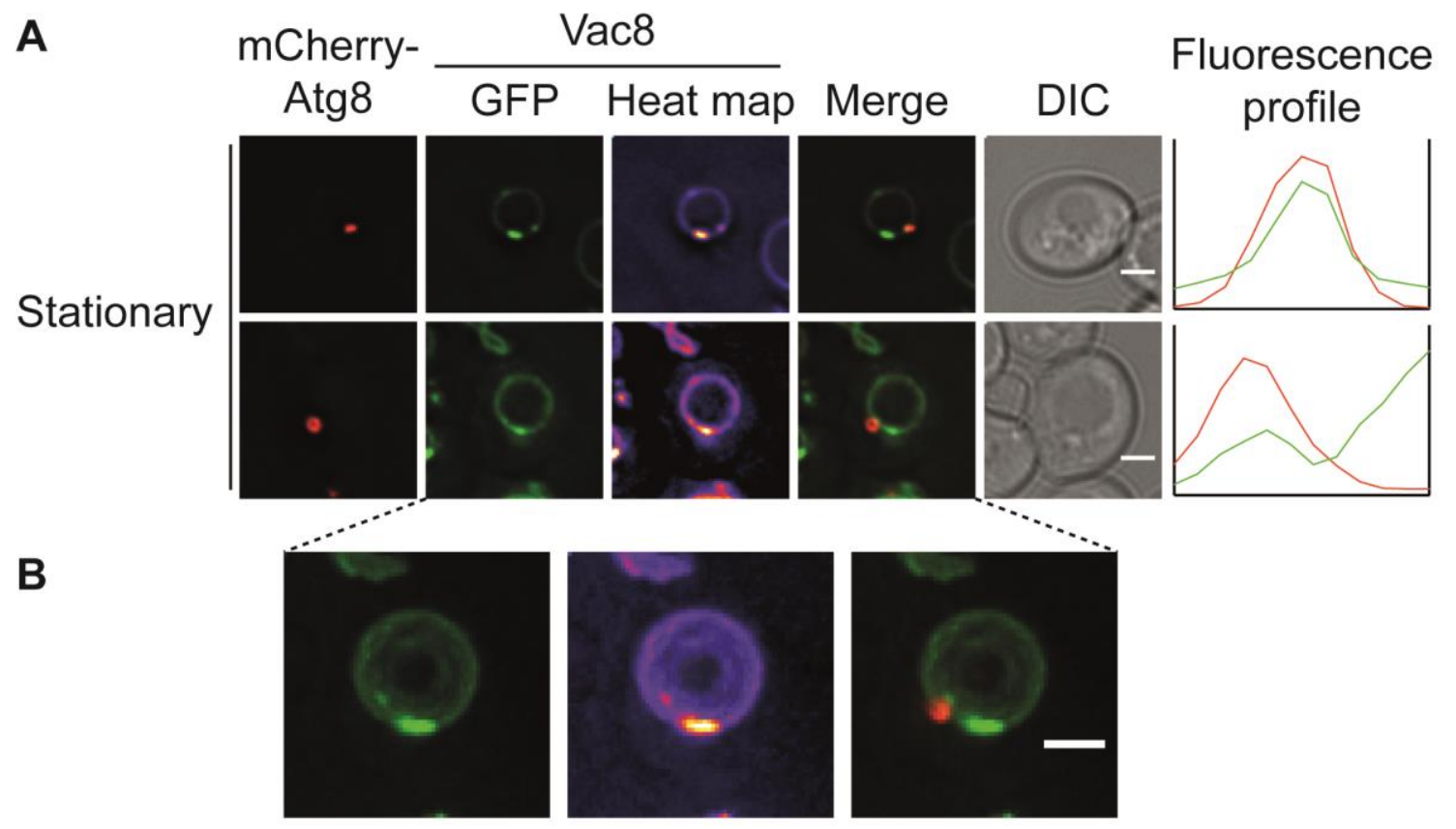

C

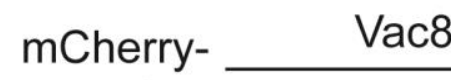

Fluorescence Atg8

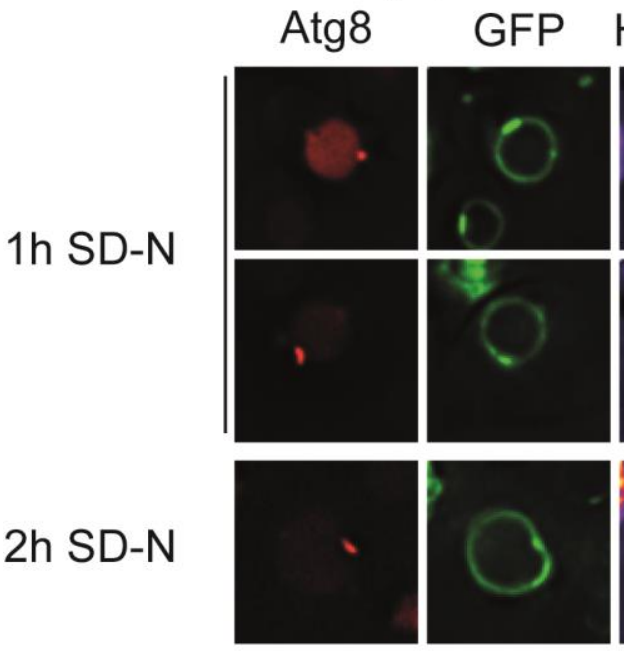

Heat map Merge

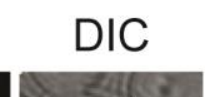
profile
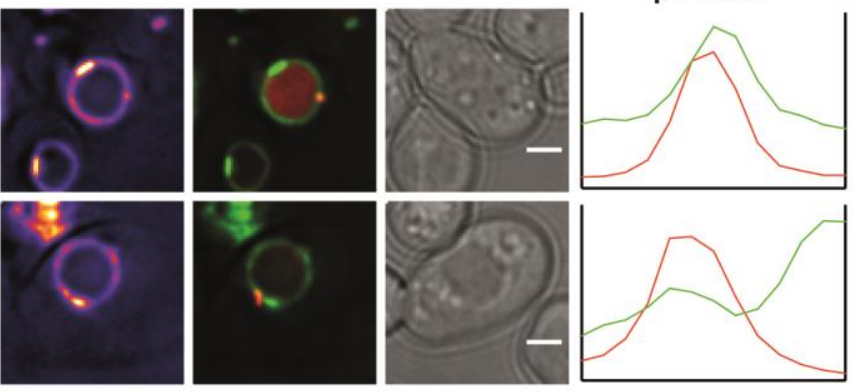

D
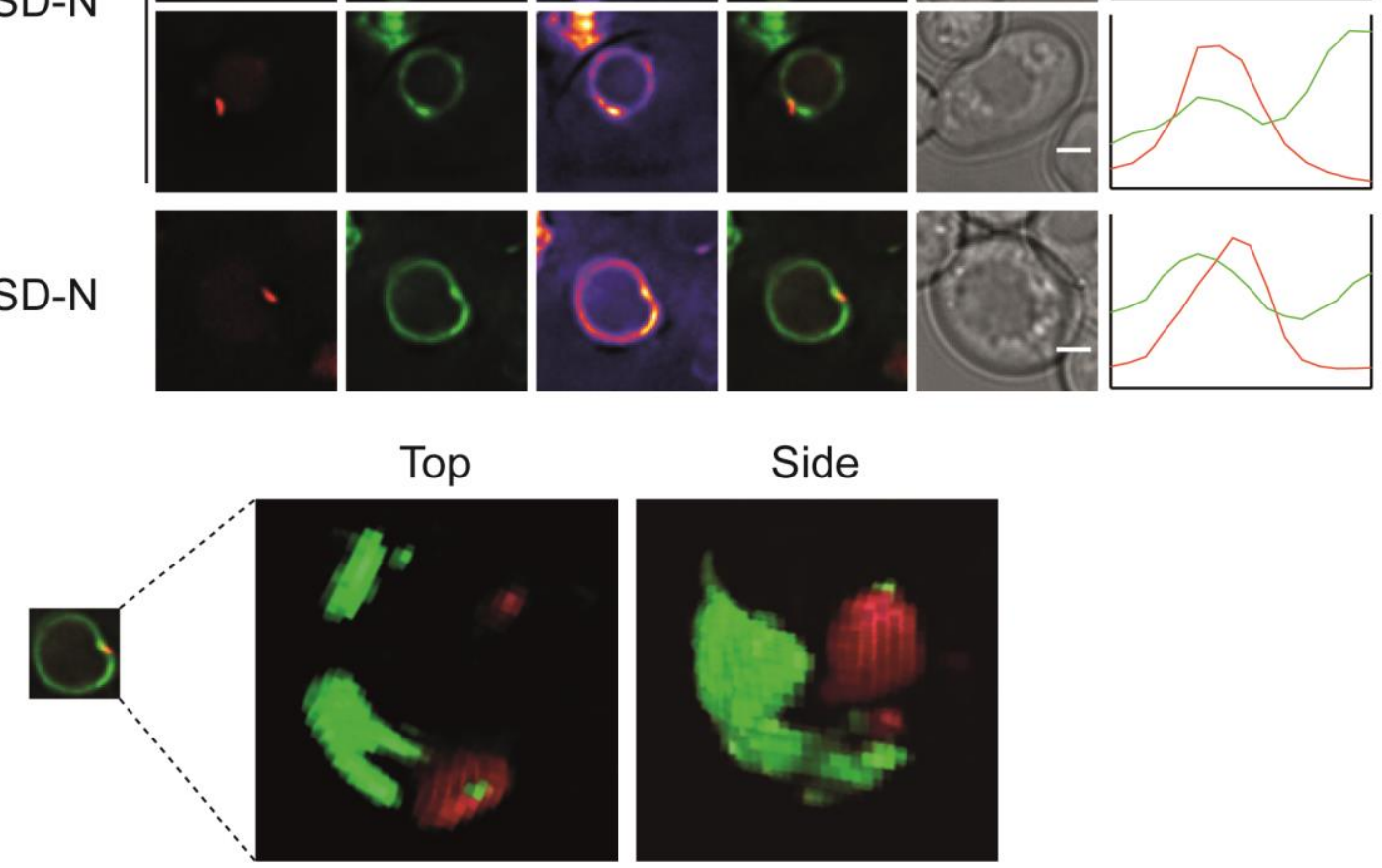

Figure 4.19: Vac8-GFP is enriched at the contact site between vacuole and phagophore

Analysis of the precise localization of Vac8-GFP at the vacuolar membrane in relation to the growing phagophore in APE1-overexpressing cells. In an atg8 vac8D strain plasmid-encoded VAC8-GFP and 
mCherry-ATG8 were expressed using their endogenous promoters. APE1 was overexpressed from the high copy pYEX-BX vector using the CUP1 promoter. Cells were grown over night in selection medium with $100 \mu \mathrm{M} \mathrm{CuSO}_{4}$. Stationary cells ( $\left.\mathrm{OD}_{600} 4-5\right)$ were analyzed directly (A and $\left.\mathbf{B}\right)$ or after 1-2 h starvation in SD-N medium (C and D) by fluorescence microscopy using the DeltaVision microscope equipped with GFP and mCherry filter sets. Scale bars represent $2 \mu \mathrm{m}$. Fluorescence profiles ( $\mathbf{A}$ and $\mathbf{C}$ ) were measured along the contact site between the phagophore and the vacuole using Fiji (Schindelin et al., 2012). Corresponding graphs were plotted with the GFP (green) and mCherry (red) intensities on different axes using GraphPad Prism ${ }^{\circledR} 7$ (GraphPad Software). Heat maps were generated by coloring the GFP channel according to its intensity with the look-up-table fire (bright (yellow) to dark (deep blue)) using Fiji. (B) 2D-projection of the intensity maxima of all taken z-stacks of the cell using SoftWoRx (Applied Precision). (C) 3D-projection of the growing phagophore using the freeware mode of the Huygens Professional X11 software (Scientific Volume Imaging).

The biologically not fully active Vac8-3xGFP showed a localization pattern at the vacuolar membrane similar to that of Vac8-GFP (Figure 4.20). Vac8-3xGFP was also found to accumulate in one bigger raft and several dot-like structures, some of which did colocalize with the mCherry-Atg8 positive phagophore. The signal of Vac8-3xGFP at the vacuolar membrane was very faint, so that enriched sites were even better detectable compared to plasmid-encoded Vac8-GFP. Even though the 3xGFP-tag interfered with the activity of Vac8, it did not seem to disturb its localization. Therefore, Vac8-3xGFP was used to support the results obtained with Vac8-GFP.

To verify that the observed accumulation of Vac8-3xGFP at the contact site of vacuole and phagophore was no artifact of the NVJ, the nucleus was stained in addition with Hoechst. For that purpose, the medium was supplemented with $12.5 \mu \mathrm{g} / \mathrm{ml}$ Hoechst $15 \mathrm{~min}$ before the cells were analyzed by fluorescence microscopy (Figure 4.20). As already speculated, the bigger rafts of Vac8-3xGFP colocalized with the Hoechst stained nucleus and therefore represented NVJs. The dot-like accumulations of Vac8-3xGFP at the contact site to the phagophore were clearly distinguishable from the NVJs and often even located on the opposite side of the vacuole (Figure 4.20).

All together these findings suggested that there was indeed a Vac8-dependent contact between the vacuolar membrane and the phagophore independent of the NVJ. 


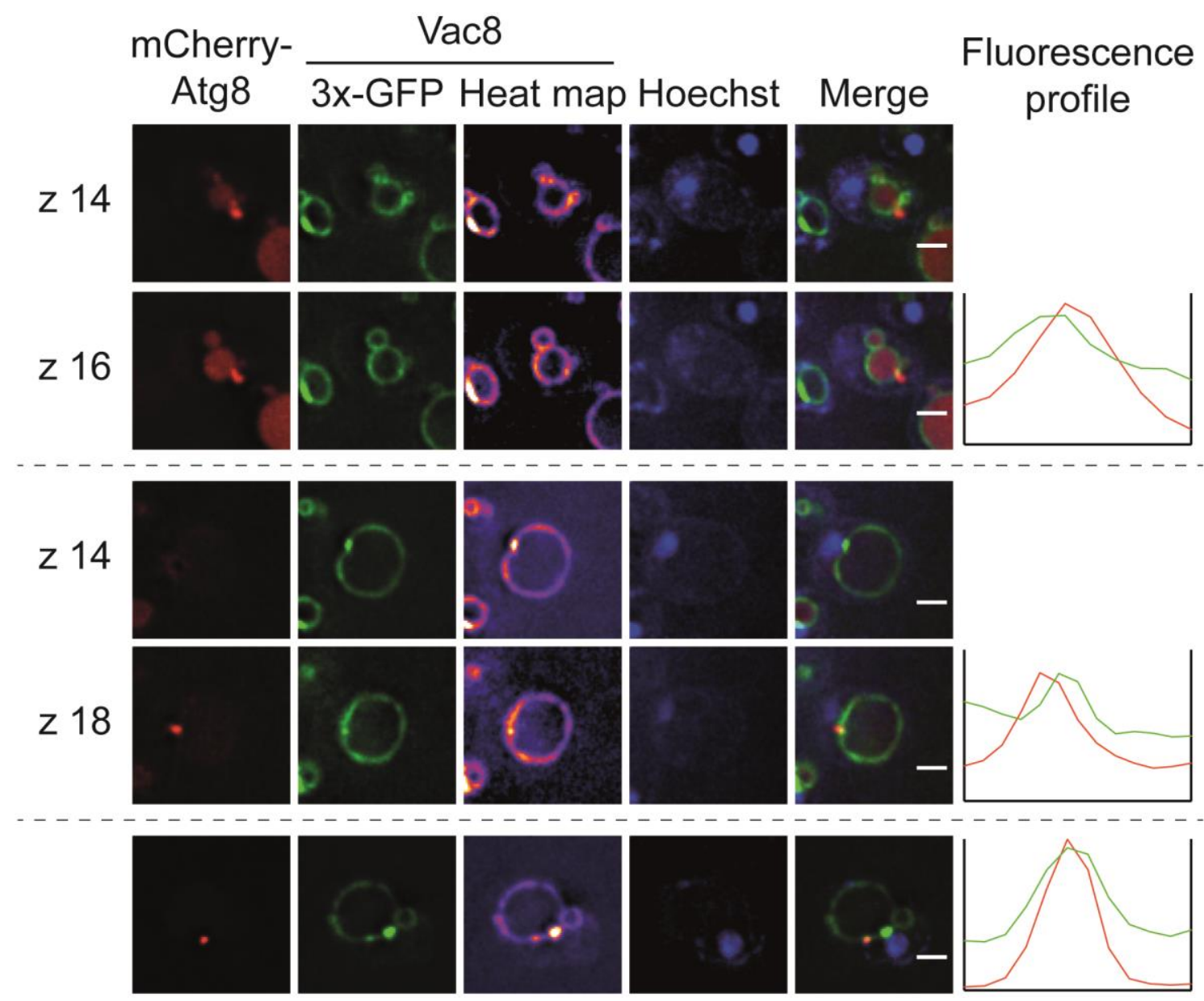

Figure 4.20: Vac8-3xGFP is enriched at the contact site of the vacuolar membrane and the phagophore independent of the NVJ

Analysis of the precise localization of Vac8-3xGFP at the vacuolar membrane in relation to the growing phagophore and the nucleus in APE1-overexpressing cells. In an atg8D VAC8-3xGFP strain plasmid-encoded mCherry-ATG8 and APE1 were expressed using the ATG8 and CUP1 promoters respectively. The nucleus was stained with Hoechst $(12.5 \mu \mathrm{g} / \mathrm{ml})$ that was added to the medium 15 min before the cells were analyzed by fluorescence microscopy. Cells were grown over night in selection medium with $100 \mu \mathrm{M} \mathrm{CuSO}_{4}$. Stationary cells $\left(\mathrm{OD}_{600} 4\right)$ were starved for $1 \mathrm{~h}$ in SD-N medium. Fluorescence microscopy was performed using the DeltaVision microscope equipped with GFP, mCherry and DAPI filter sets. Scale bars represent $2 \mu \mathrm{m}$. Fluorescence profiles were measured along the contact site between the phagophore and the vacuole using Fiji (Schindelin et al., 2012). Corresponding graphs were plotted with the GFP (green) and mCherry (red) intensities on different axes using GraphPad Prism ${ }^{\circledR} 7$ (GraphPad Software). Heat maps were generated by coloring the GFP channel according to its intensity with the look-up-table fire (bright (yellow) to dark (deep blue)) using Fiji. $\mathrm{z}$ indicates the number of the displayed focal plane.

\subsubsection{Vac8 participates in the formation of the phagophore}

Since Vac8 localized to the contact site between vacuole and phagophore (chap. 4.2.5.1), it was very likely that it also participated in the formation of the PAS as well as the phagophore and/or played a role in maintaining the contact site of vacuole and phagophore. Vac8 was also a potential interaction partner of Atg21 that could be responsible for the localization of Atg21 to this contact site. To further verify this 
hypothesis, additional experiments were performed using the APE1-overexpression assay.

Initially, the effect of the VAC8 deletion on the formation of the PAS and the phagophore was analyzed and compared to that of the ATG21 deletion. Therefore, $A T G 21$ or VAC8 were deleted in the atg8 APE1-RFP (WT) strain. To visualize the

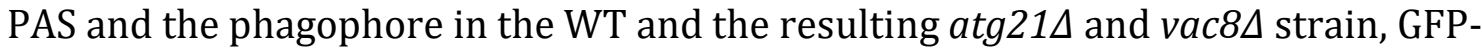
ATG8 and APE1 were expressed from plasmids as described before (chap. 4.2.1). Stationary cells were starved for $1 \mathrm{~h}$ in SD-N medium and analyzed using fluorescence microscopy. As described for the optimization of the APE1overexpression assay (chap. 4.2.1), the number of GACs (Figure 4.21 B) and their colocalization with GFP-Atg8 (Figure 4.21 A) were determined. The observed GFPAtg8 structures were again grouped in dot-like (PAS), slightly elongated and cup-like (Phagophore). For all structures as well as the sum of all structures (Total) the colocalization rates were calculated (Figure $4.21 \mathrm{~A}$ ).

In total $35.96 \pm 1.82 \%$ of the $0.68 \pm 0.02$ GACs per cell in the WT strain colocalized with GFP-Atg8 structures. The colocalizing structures could be grouped in $23.07 \pm 1.71 \%$ that colocalized with dot-like structures, $8.75 \pm 1.02 \%$ with elongated structures and $4.14 \pm 0.85 \%$ with cup-like structures. The calculated colocalization rates of the WT strain as well as the number of GACs per cell were comparable to those observed during the optimization of the APE1-overexpression assay (chap. 4.2.1) and indicated that the assay was working.

Interestingly, the number of GACs per cell did not change for the atg21 $\Delta$ strain but significantly increased in the vac8s strain $(0.8 \pm 0.02$ per cell). This suggested that the deletion of VAC8 might influence the formation of the GACs, so that smaller and therefore more complexes are formed per cell. The size of the GACS was not measured in this study and could therefore not be evaluated.

For both, the $\operatorname{atg} 21 \Delta$ and the vac8s strain, a significant reduction of GFP-Atg8 positive elongated and cup-like structures was observed. While for the atg21 $\mathrm{strain}$ still $1.64 \pm 0.43 \%$ of the GACs were engulfed by a phagophore, almost no phagophores were observed for the vac8 strain ( $0.43 \pm 0.23 \%$ per GAC). This was also true for the slightly elongated structures of GFP-Atg8. Here, $2.58 \pm 0.33 \%$ of the 
GACs in the $\operatorname{atg} 21 \Delta$ strain compared to $1.8 \pm 0.45 \%$ in the vac8D strain colocalized with elongated structures of GFP-Atg8.

A

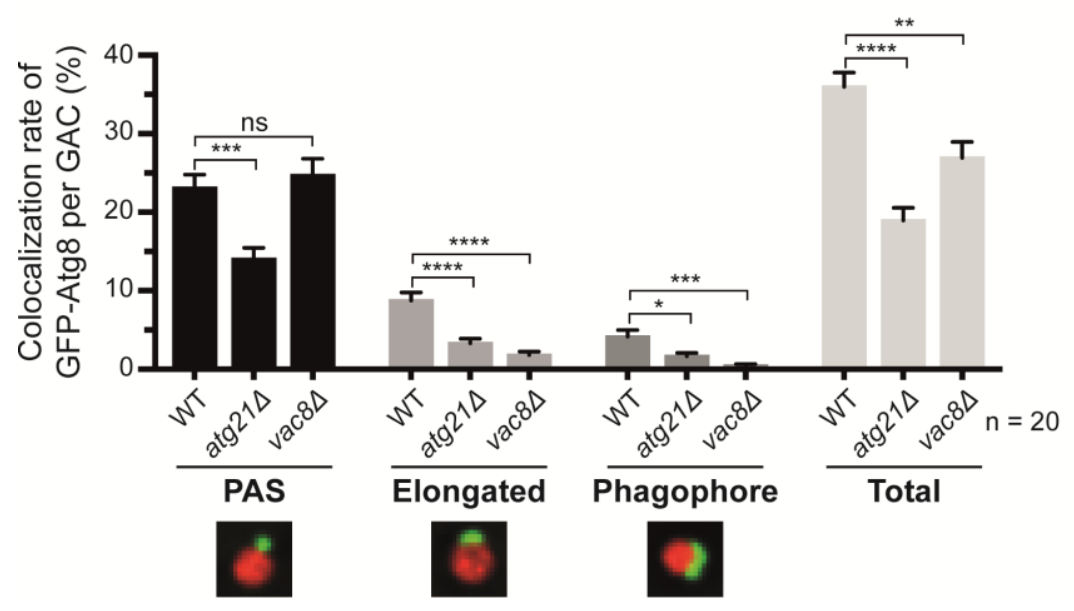

B

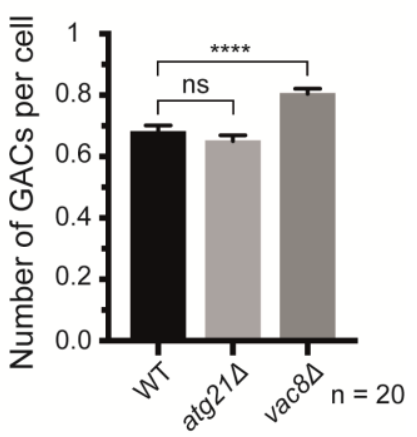

Figure 4.21: Deletion of $A T G 21$ and VAC8 significantly reduces the formation of phagophores

Analysis of the effect of the deletion of $A T G 21$ and $V A C 8$ on the formation of the phagophore using

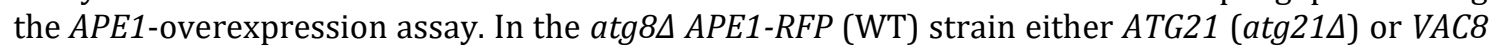
(vac8D) was deleted. In these strains, plasmid-encoded GFP-ATG8 and APE1 were expressed using the ATG 8 and CUP1 promoter respectively. The cells were grown over night in selection medium with $100 \mu \mathrm{M} \mathrm{CuSO}_{4}$. Stationary cells $\left(\mathrm{OD}_{600} 4-5\right)$ were starved for $1 \mathrm{~h}$ in SD-N medium and analyzed by fluorescence microscopy using the DeltaVision microscope equipped with GFP and mCherry filter sets. In two independent experiments with at least 998 counted cells per strain, the number of GACs per cell (B) and their colocalization with GFP-Atg8 (A) was determined per image. The shape of the colocalizing GFP-Atg8 signal was grouped into dot-like (PAS), slightly elongated and cup-like (Phagophore) structures. The colocalization rate of each group and of the sum of all groups (Total) was calculated. $\mathrm{n}$ indicates the number of analyzed images per strain. Statistical relevance was determined using the unpaired two-tailed t-test. Error bars indicate SEM and asterisks indicate pvalues: ns, not significant $P>0.05 ;{ }^{*} P<0.05 ;{ }^{* *} P<0.01$; ${ }^{* * *} P<0.001$; ${ }^{* * * *} P<0.0001$

Remarkably, the deletion of VAC8 did not reduce the number of GACs colocalizing with dot-like structures of GFP-Atg8. With $24.7 \pm 2.1 \%$ the colocalization rate increased slightly but not significantly in the vac84 strain and was therefore comparable to that of the WT strain. In contrast, the $\operatorname{atg} 21 \Delta$ strain showed a significantly reduced colocalization rate compared to the WT strain with $14.01 \pm 1.45 \%$ of the GACs colocalizing with dot-like structures of GFP-Atg8. Consequently, the colocalization rate of the in total observed GFP-Atg8 structures in the $\operatorname{atg} 21 \Delta$ strain $(18.95 \pm 1.61 \%)$ was stronger reduced in comparison to that of the vac8 8 strain $(26.93 \pm 2.03 \%)$. These findings correlated with the reported role of Atg21 in the recruitment and subsequent lipidation of Atg8 at the PAS (Juris et al., 2015).

All together the obtained results suggested that Vac8 is not essential for PAS formation but is a crucial factor for the elongation of the phagophore. In contrast, 
Atg21 is more important for the formation of the PAS. In the atg21D strain less Atg8 was recruited to the PAS and as a consequence less phagophores were formed.

\subsubsection{Upon deletion of VAC8 the contact between vacuolar membrane and phagophore is affected}

When evaluating the localization and formation of GFP-Atg8 positive structures in the vac8D strain (chap. 4.2.5.2), it seemed as if some of those structures were formed without any contact to the vacuole. This observation provided a first evidence that Vac8 might be essential for the formation and/or maintenance of a contact site between vacuolar membrane and phagophore. To verify these findings, the precise localization of the PAS in relation to the vacuole was evaluated using the APE1-overexpression assay.

To visualize the vacuolar membrane, a PHO8 gene N-terminally tagged with $3 x \operatorname{tagBFP}$ (vac-BFP) and controlled by the PGK1 promoter was introduced into the LEU2 locus of the strains analyzed above (chap. 4.2.5.2). The repressible vacuolar alkaline phosphatase Pho8 is an integral vacuolar membrane protein with its active site directed to the inner side of the vacuole (Klionsky and Emr, 1989). It is often used as a marker protein for the vacuolar membrane. The resulting strains expressed APE1-mRFP and 3xtagBFP-PHO8 from the chromosome. In addition, they were transformed with the GFP-ATG8 and APE1 expressing plasmids. The strains were cultivated according to the previous experiment (chap. 4.2.5.2) and analyzed by fluorescence microscopy using the DeltaVision microscope (Figure 4.22 A).

Again, the number of mRFP-pApe1 positive GACs per cell and their colocalization with GFP-Atg8 positive structures was determined (Figure 7.2) and compared to the results of chap. 4.2.5.2. As the observed colocalization rates of the WT strain with integrated 3xtagBFP-PHO8 were comparable to those observed in the WT strain without 3xtagBFP-PHO8 (chap. 4.2.5.2), it was assumed that 3xtagBFP-Pho8 did not influence the formation of the phagophore and could therefore be used to visualize the vacuolar membrane.

While in the respective WT and atg21 strain GFP-Atg8 structures at the GACs were predominantly in contact with the vacuolar membrane, many of the GFP-Atg8 structures in the vac84 strain had no contact to the vacuole. This was validated using 3D-projections of the z-stacks of the analyzed cells. Indeed, there was no contact 
found between phagophore and vacuole in upper or lower layers of the cell for the respective structures in the vac8D strain (Figure $4.22 \mathrm{~B}$ ). However, some of the GFP-Atg8 positive structures in the vac80 strain still had a contact to the vacuolar membrane. In contrast to the WT and the $\operatorname{atg} 21 \Delta$ strain, often more than one GFP-Atg8 structure colocalized with the same GAC in the vac8D strain.

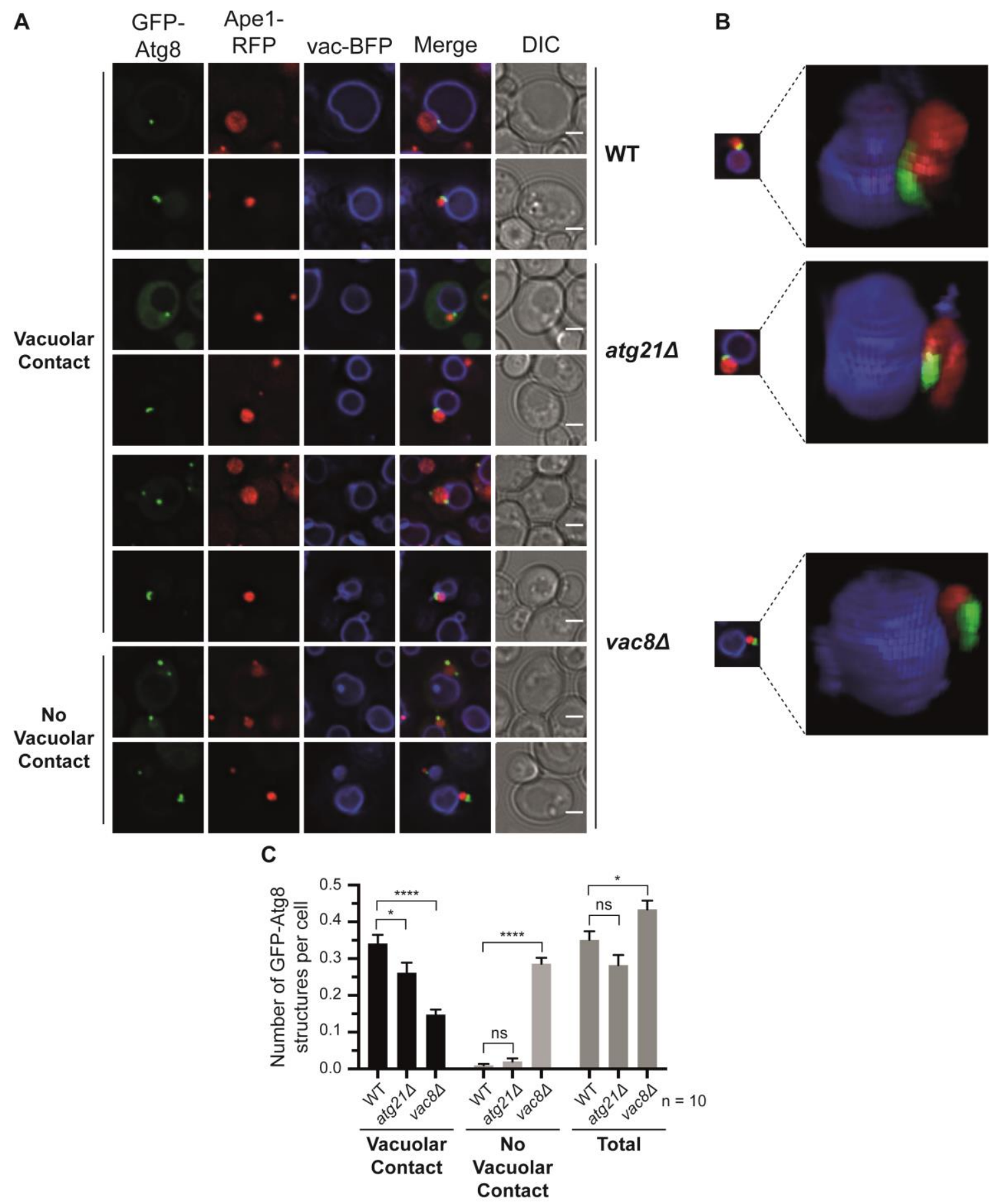

Figure 4.22: Upon deletion of VAC8 the contact between the PAS/phagophore and the vacuolar membrane is highly reduced

Analysis of the changes in the localization of the PAS/phagophore at the GACs in relation to the vacuolar membrane upon deletion of ATG21 or VAC8. (A) In the atg8D APE1-RFP 3xtagBFP-PHO8 
(WT) strain either ATG21 (atg214) or VAC8 (vac84) was deleted. In the WT and the resulting strains plasmid-encoded GFP-ATG8 and APE1 were expressed using the ATG8 and CUP1 promoter respectively. The cells were grown over night in selection medium with $100 \mu \mathrm{M} \mathrm{CuSO}_{4}$. Stationary cells $\left(\mathrm{OD}_{600} 4-5\right)$ were starved in SD-N medium for $1 \mathrm{~h}$ and analyzed by fluorescence microscopy using the DeltaVision microscope equipped with FITC, TRITC and DAPI filter sets. (B) 3D-projections of the GACs in contact with the vacuole and the growing phagophore using the freeware mode of the Huygens Professional X11 software (Scientific Volume Imaging). (C) For each strain the number of GFP-Atg8 structures per cell was counted (Total) and grouped into contact with the vacuolar membrane and no contact. In total, 10 images (n) with at least 540 cells per strain were analyzed. Statistical relevance was determined using the unpaired two-tailed t-test. Error bars indicate SEM and asterisks indicate p-values: ns, not significant $P>0.05$; ${ }^{*} P<0.05$; ${ }^{* * *} P<0.0001$. $\mathrm{n}$ indicates the number of analyzed images.

Interestingly, in all analyzed strains some of the GFP-Atg8 dots did not colocalize with the GACs. This could be autophagosomes forming independent of the GACs or the formation of a PAS at a GAC that is not detectable with fluorescence microscopy. It had to be considered that most of the APE1 was expressed from plasmid without the RFP-tag. Therefore, GACs formed without the Ape1-RFP would not be detectable by fluorescence microscopy. These structures have not been of interest for the previous experiments (chap. 4.2.1) but were now included into the evaluations, as many of those GFP-Atg8 structures showed no contact to the vacuolar membrane in the vac80 strain.

To get an impression of how many GFP-Atg8 structures were in contact with the vacuole and how many were not, the number of structures per cell was counted (Total) and grouped into those with vacuolar contact and those without (Figure $4.22 \mathrm{C}$ ). Those structures that did not colocalize with the GACs were also included. Indeed, the main portion of GFP-Atg8 structures, almost $100 \%$ of the in total counted structures, was in contact with the vacuolar membrane in the WT $(0.34 \pm 0.02$ per cell) and the $\operatorname{atg} 21 \Delta$ strain $(0.26 \pm 0.03$ per cell). Only very rarely GFP-Atg8 structures did not colocalize with the vacuolar membrane in both strains ( 0.02 or less structures per cell). This changed significantly in the vac8D strain. Here, only about one third ( $0.15 \pm 0.01$ per cell) of all counted GFP-Atg8 structures (Total: $0.43 \pm 0.02$ per cell) were in contact with the vacuolar membrane while the other two thirds $(0.29 \pm 0.01$ per cell $)$ did not colocalize with the vacuole. These findings suggested that Vac8 was indeed responsible for the formation of the contact between the vacuolar membrane and the phagophore. 


\subsubsection{Vac8 and Atg21 co-precipitate in the same complex}

Since the deletion of ATG21 did not influence the contact between vacuole and phagophore, it was unlikely that it is the main interaction partner of Vac8 if it even interacted with Vac8. The GFP-TRAP approach was used to analyze whether Atg21 and Vac8 interact or are at least part of the same complex.

Therefore, Vac8 was chromosomally tagged with a $6 x H A-\operatorname{tag}$ in an atg $21 \Delta$ strain. The Vac8-6xHA fusion protein was used as the prey in the GFP-TRAP experiment. As described before, the plasmid-encoded bait (GFP and GFP-ATG21) was overexpressed using the MET25 promoter. GFP alone served as a control to detect potential unspecific binding of Vac8-6xHA to GFP. Figure 4.23 representatively shows the result of one of the two independently performed GFP-TRAP experiments.

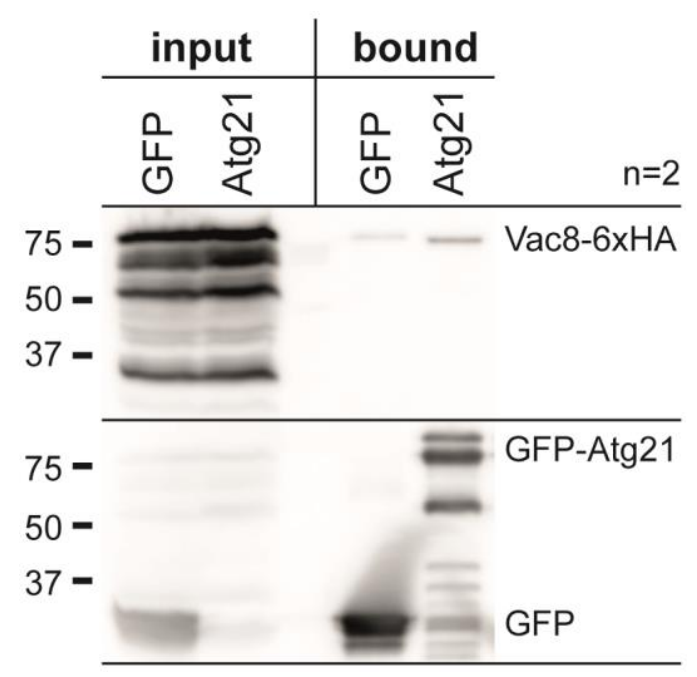

Figure 4.23: Vac8 precipitates together with GFP-Atg21 using the GFP-TRAP approach

Analysis of the interaction of Atg21 and Vac8 using the GFP-TRAP approach. In an atg21 $V A C 8-6 x H A$ strain plasmid-encoded GFP or GFP-ATG21 were overexpressed as the baits using the MET25 promoter. Cells were grown in selection medium without L-methionine to early stationary phase $\left(\mathrm{OD}_{600} 2\right)$, osmotically lysed and incubated with GFP-TRAP beads. Input and bound fractions of two independent experiments (n) were analyzed by Western-Blot. The molecular weight marker is depicted in $\mathrm{kDa}$.

In the GFP-TRAP experiments part of Vac8-6xHA was isolated in complex with Atg21-GFP. This suggested that Atg21 and Vac8 were indeed at least components of the same complex. However, also some unspecific binding of Vac8-6xHA to GFP alone was detected. Further optimization of the GFP-TRAP approach and additional experiments are required to reveal whether there is a direct interaction between both proteins. 


\subsubsection{Formation of a specialized vacuolar membrane domain at the contact site between vacuole and phagophore}

The previous experiments revealed the formation of a Vac8-dependent vacuolephagophore contact site (chap. 4.2.5). The enrichment of Vac8 further suggested that the contact site might coincide with the formation of a specialized domain or even a lipid raft at the vacuolar membrane.

Lipid rafts are defined as dynamic nanoscale subcompartments of the membrane enriched in sterols and sphingolipids. They seem to organize proteins in the membrane and to play a role in post-Golgi trafficking, endocytosis and other cellular processes (Simons and Sampaio, 2011). It has been suggested that the high affinity of sterols for lipids with saturated and longer acyl chains (e.g. sphingolipids) drives the formation of a liquid-ordered ( $\mathrm{L}_{0}$ ) microdomain. Lipids which are excluded from that microdomain form liquid-disordered $\left(\mathrm{Ld}_{\mathrm{d}}\right)$ regions in the membrane. Both domains can coexist and lead to a phase separation (Helms and Zurzolo, 2004). Besides the molecular interaction of lipids, protein-lipid interactions seem to be able to stabilize or even form lipid rafts. Usually, transmembrane proteins are excluded from $L_{o}$ domains while palmitoylation seems to increase the affinity of proteins for lipid rafts (Simons and Sampaio, 2011). The concept of lipid rafts is frequently discussed since it is mainly based on in vitro model systems. However, Toulmay and Prinz (2013) were able to show the formation of large lipid domains in the vacuolar membrane of late stationary yeast cells (2 days). They found that proteins segregated into $\mathrm{L}_{o}$ and $\mathrm{L}_{d}$ domains.

Vph1 is an integral membrane protein of the vacuole and a component of the vacuolar proton-translocating ATPase (Manolson et al., 1992). It was identified as a marker for the $L_{d}$ domain (Toulmay and Prinz, 2013). Interestingly, Vph1 is excluded from those regions of the vacuole that form the NVJ (Dawaliby and Mayer, 2010). According to this, Vph1 was a perfect candidate to analyze whether the vacuole-phagophore contact site is formed at a specialized membrane domain of the vacuole. 


\subsubsection{Vph1 is excluded from the contact site between vacuole and phagophore}

Whether Vph1 was excluded from the vacuole-phagophore contact site, as it is described for the NVJ (Dawaliby and Mayer, 2010), was tested using the APE1overexpression assay. For this, VPH1 was chromosomally tagged with GFP in an atg8 8 strain. In the resulting strain plasmid-encoded mCherry-ATG8 and APE1 were expressed as described before (chap. 4.2 .1 and 4.2.2). Stationary cells were either analyzed directly by fluorescence microscopy or after starvation for $1-2 \mathrm{~h}$ in SD-N medium (Figure 4.24).

Vph1-GFP was evenly distributed on major parts of the vacuolar membrane. Beside this, some Vph1-GFP negative raft-like domains were identified (Figure 4.24 A). Usually, Vph1 was excluded from one bigger domain however also one or more smaller exclusions could be observed. The exclusions of Vph1-GFP were observed in stationary as well as starved cells and therefore seemed to be independent of the growth conditions. In a representative cell the length of the observed Vph1-GFP negative domains was measured (Figure $4.24 \mathrm{~B}$ ). The length of the bigger exclusion $(1.17 \mu \mathrm{m})$ was almost two times bigger than that of the smaller domain $(0.65 \mu \mathrm{m})$. The observed localization of Vph1-GFP seemed to be inversely correlated to the localization of Vac8-GFP (Figure 4.19). Therefore, the bigger Vph1 negative domains could represent part of the Vac8-GFP enriched NVJs.

Some of the smaller exclusions were frequently colocalized with mCherry-Atg8 positive phagophores, suggesting that there was indeed an exclusion of Vph1 from the vacuole-phagophore contact site. This became most obvious looking at fluorescence profiles of the contact site (Figure 4.24 A and B). Here, the intensity peak of the mCherry-Atg8 signal always correlated with a drastic decrease of the signal intensity of Vph1-GFP. 3D-projections of all imaged focal planes of a cell revealed that there was no direct contact between the smaller and bigger Vph1-GFP negative domains. In agreement with the observations for Vac8-3xGFP (Figure 4.20 ), this indicated that the contact site between vacuole and phagophore was independent of the NVJ. 
A
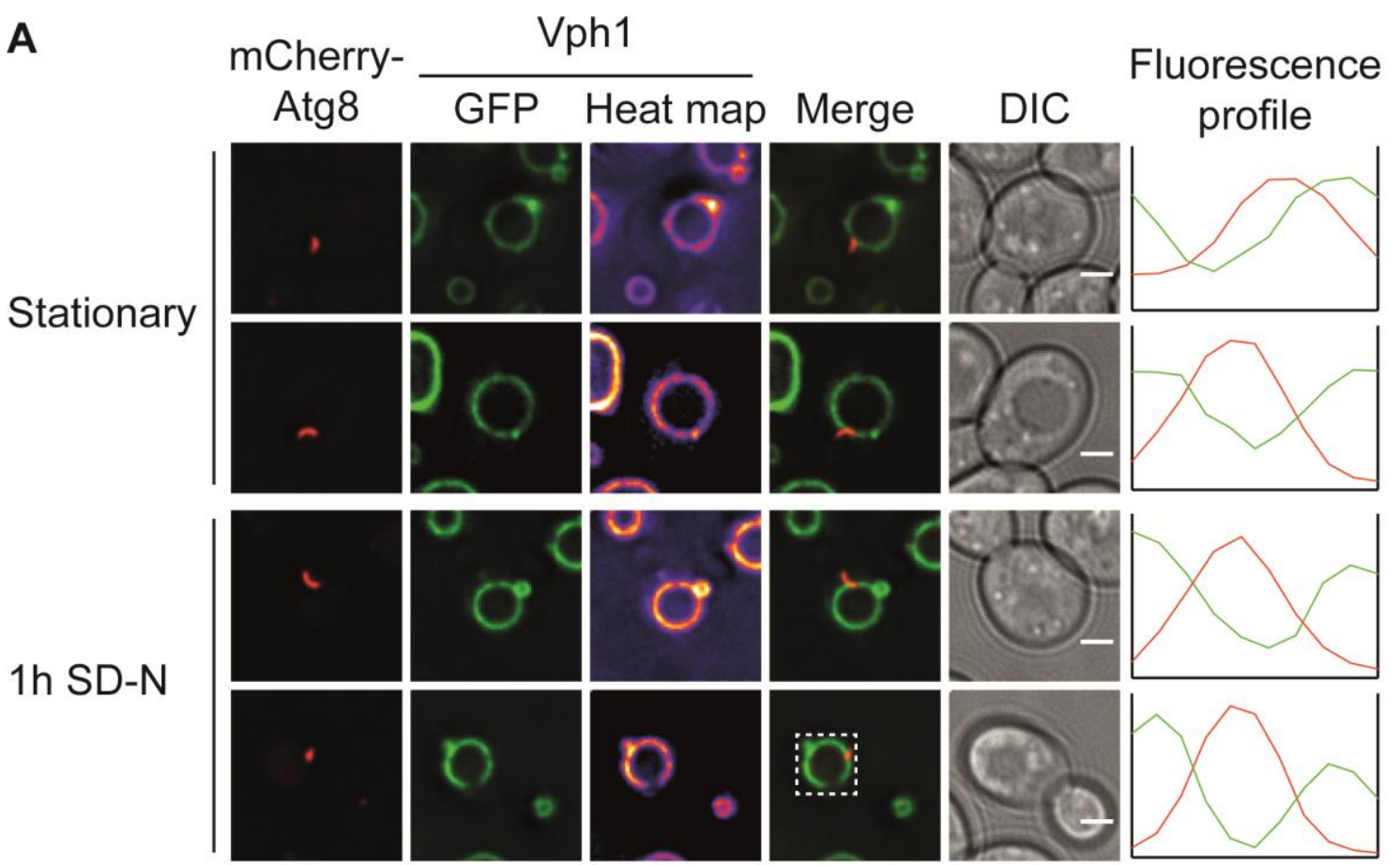

B

Fluorescence profile

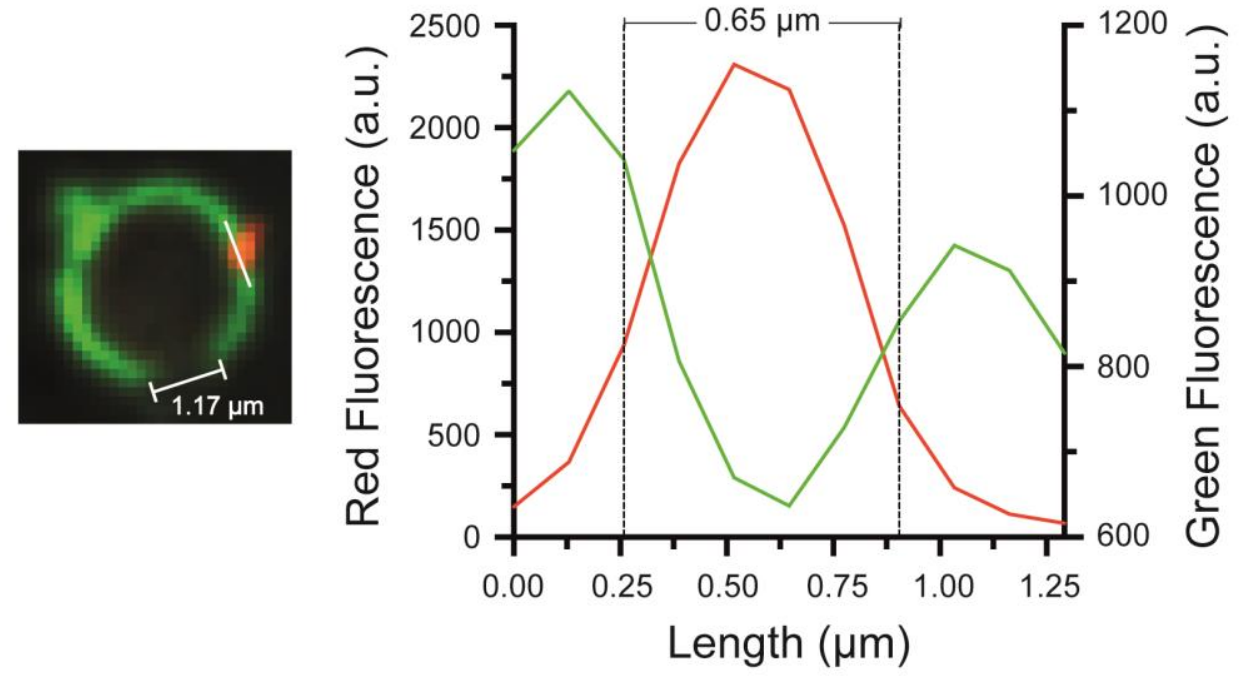

C

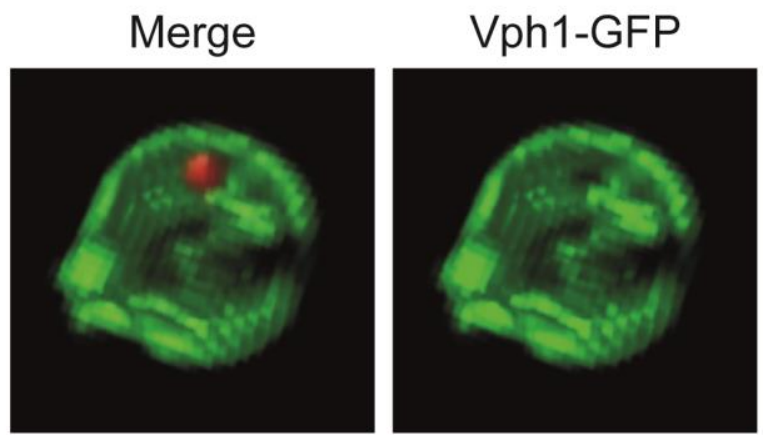

Figure 4.24: Vph1-GFP is excluded from the contact site between vacuole and phagophore

Analysis of the precise localization of Vph1-GFP at the vacuolar membrane in relation to the growing phagophore in APE1-overexpressing cells. In an atg8 VPH1-GFP strain, plasmid-encoded mCherryATG8 and APE1 were expressed using the ATG8 and the CUP1 promoter respectively. Cells were grown over night in selection medium with $100 \mu \mathrm{M} \mathrm{CuSO}_{4}$. Stationary cells $\left(\mathrm{OD}_{600} 5\right)$ were directly 
analyzed by fluorescence microscopy or starved for $1 \mathrm{~h}$ in SD-N medium. For fluorescence microscopy the DeltaVision microscope equipped with GFP and mCherry filter sets was used. Scale bars represent $2 \mu \mathrm{m}$. Fluorescence profiles (A and $\mathbf{B}$ ) were measured along the contact site between the phagophore and the vacuole (white bar in B) using Fiji (Schindelin et al., 2012). Corresponding graphs were plotted with the GFP (green) and mCherry (red) intensities on different axes using GraphPad Prism ${ }^{\circledR} 7$ (GraphPad Software). Heat maps were generated by coloring the GFP channel according to its intensity with the look-up-table fire (bright (yellow) to dark (deep blue)) using Fiji. (C) 3D-projection of Vph1-GFP at the vacuolar membrane and the mCherry-Atg8 positive phagophore using the freeware mode of the Huygens Professional X11 software (Scientific Volume Imaging).

To validate that the bigger exclusions of Vph1-GFP corresponded to NVJs, the above described experiment was repeated with an additional staining of the nucleus using Hoechst. The medium was supplemented with $12.5 \mu \mathrm{g} / \mathrm{ml}$ Hoechst $15 \mathrm{~min}$ before the cells were analyzed by fluorescence microscopy (Figure 4.25).

The Hoechst stained nuclei were indeed observed in close proximity to Vph1-GFP negative domains at the vacuolar membrane defining them as NVJs. In analogy to the previous experiment, mCherry-Atg8 positive structures also colocalized with Vph1-GFP excluded regions of the vacuolar membrane. These regions were clearly distinguishable from the Vph1-GFP negative NVJs or even located on the opposite side of the vacuole (Figure 4.25 C and D). 2D- and 3D-projections of all imaged focal planes of the cells again validated that there was no contact between the individual Vph1-GFP exclusions.

Together these findings proved that a specialized Vac8-dependent membrane domain is formed at the contact site of vacuole and phagophore. This domain excluded Vph1-GFP, a marker for Ld domains, suggesting the formation of a lipid raft (Lo domain). The palmitoylation of Vac8 supports the hypothesis of a raft formation since palmitoylation increases the affinity of proteins for lipid rafts (Simons and Sampaio, 2011). However, to verify that lipids contribute to this specialized domain further experiments are needed. 
A
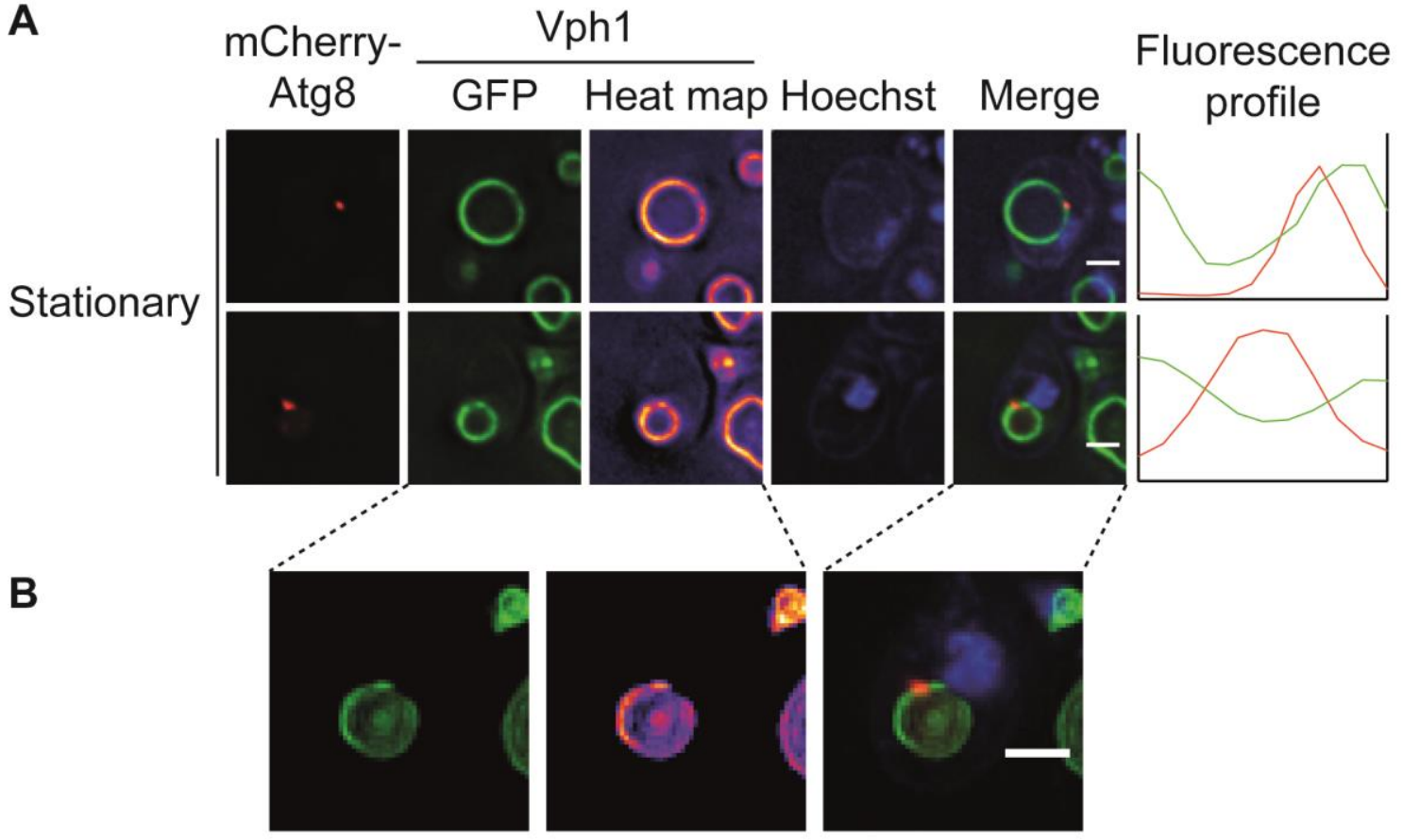

C

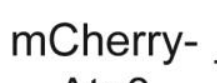

Vph1

2h SD-N

$$
\text { Atg8 }
$$
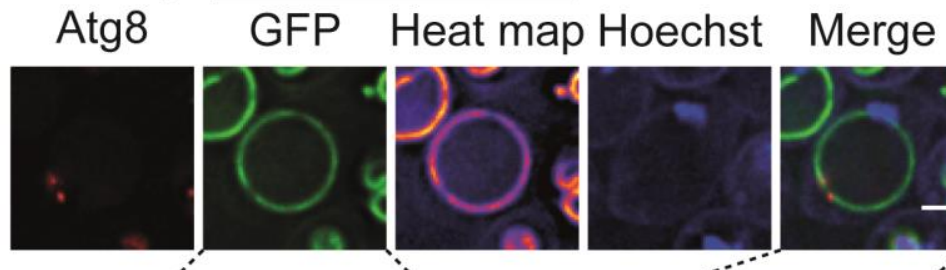

Fluorescence

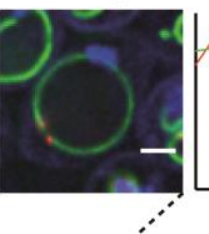
profile

D
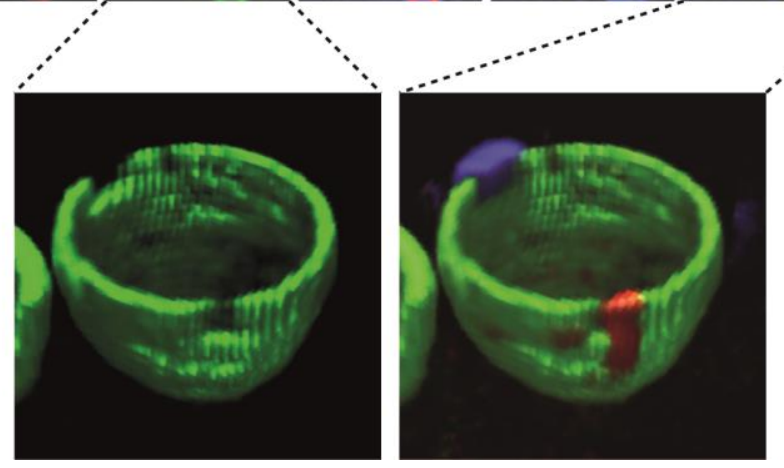

Figure 4.25: The exclusion of Vph1-GFP from the contact site between vacuole and phagophore is independent of the NVJ

Analysis of the precise localization of Vph1-GFP exclusions in relation to the nucleus in APE1overexpressing cells. In an atg8D VPH1-GFP strain, plasmid-encoded mCherry-ATG8 and APE1 were expressed using the ATG8 and the CUP1 promoter respectively. The nucleus was stained with Hoechst $(12.5 \mu \mathrm{g} / \mathrm{ml})$ that was added to the medium $15 \mathrm{~min}$ before the cells were analyzed by fluorescence microscopy. Cells were grown over night in selection medium with $100 \mu \mathrm{M} \mathrm{CuSO}$. Stationary cells (OD600 5) were directly analyzed by fluorescence microscopy or starved for $2 \mathrm{~h}$ in SD-N medium. For fluorescence microscopy the DeltaVision microscope equipped with DAPI, GFP and mCherry filter sets was used. Scale bars represent $2 \mu \mathrm{m}$. Fluorescence profiles (A and $\mathbf{C}$ ) were measured along the contact site between the phagophore and the vacuole using Fiji (Schindelin et al., 2012). Corresponding graphs were plotted with the GFP (green) and mCherry (red) intensities on different axes using GraphPad Prism ${ }^{\circledR} 7$ (GraphPad Software). Heat maps were generated by coloring the GFP channel according to its intensity with the look-up-table fire (bright (yellow) to dark (deep blue)) using Fiji. (B) 2D-projection of the intensity maxima of all taken z-stacks of the cell using SoftWoRx (Applied Precision). (D) 3D-projection of Vph1-GFP at the vacuolar membrane and the 
mCherry-Atg8 positive phagophore using the freeware mode of the Huygens Professional X11 software (Scientific Volume Imaging).

\subsubsection{Further characterization of the identified vacuolar membrane domain}

To further characterize the nature of the observed vacuolar membrane domain at the vacuole-phagophore contact site, additional experiments were performed using the APE1-overexpression assay. To analyze whether the contact site represented a general diffusion barrier for non-participating proteins, the 3xtagBFP-Pho8 fusion protein was again chosen as a representative for a small vacuolar membrane integral protein (chap. 4.2.5.3; (Klionsky and Emr, 1989)). Only its small N-terminal portion together with the 3xtagBFP-tag is exposed to the cytosol while its active site is oriented towards the vacuolar lumen. The experiment was performed using the atg8 $3 x$ tagBFP-PHO8 strain, where 3xtagBFP-PHOB controlled by the PGK1 promoter was integrated into the LEU2 locus (chap. 4.2.5.3). In that strain, plasmidencoded GFP-ATG8 and APE1 were expressed as described before (chap. 4.2.1). Stationary cells were either analyzed directly by fluorescence microscopy or after starvation for $1 \mathrm{~h}$ in SD-N medium (Figure 4.26).

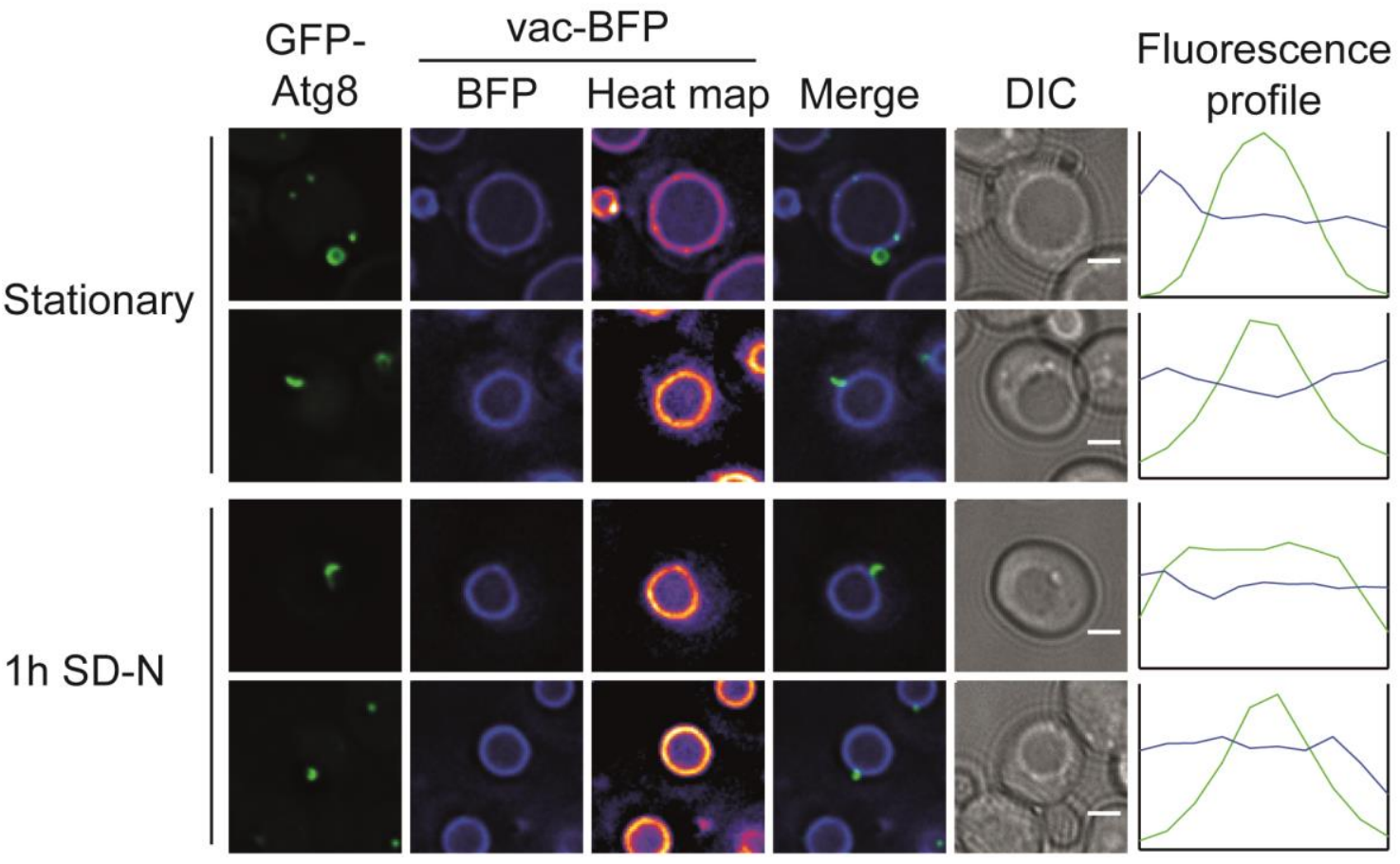

Figure 4.26: Pho8 is not excluded from the contact site between vacuole and phagophore

Analysis of the distribution of 3xtagBFP-Pho8 (vac-BFP) at the vacuolar membrane in APE1overexpressing cells. In an atg $8 \triangle 3 x \operatorname{tagBFP-PHO8}$ strain, plasmid-encoded GFP-ATG8 and APE1 were expressed using the $A T G 8$ and the CUP1 promoter respectively. Cells were grown over night in selection medium with $100 \mu \mathrm{M} \mathrm{CuSO}{ }_{4}$. Stationary cells $\left(\mathrm{OD}_{600} 5\right)$ were directly analyzed by 
fluorescence microscopy or starved for $1 \mathrm{~h}$ in SD-N medium. For fluorescence microscopy the DeltaVision microscope, equipped with DAPI and FITC filter sets, was used. Scale bars represent $2 \mu \mathrm{m}$. Fluorescence profiles were measured along the contact site between the phagophore and the vacuole using Fiji (Schindelin et al., 2012). Corresponding graphs were plotted with the GFP (green) and tagBFP (blue) intensities on different axes using GraphPad Prism ${ }^{\circledR} 7$ (GraphPad Software). Heat maps were generated by coloring the tagBFP channel according to its intensity with the look-up-table fire (bright (yellow) to dark (deep blue)) using Fiji.

In contrast to Vph1-GFP, 3xtagBFP-Pho8 was evenly distributed at the vacuolar membrane corresponding to the observed distribution in previous experiments (chap. 4.2.5.3). Measured fluorescence profiles along the vacuole-phagophore contact site supported the visual observations (Figure 4.26). There were no changes in the fluorescence intensity of 3xtagBFP-Pho8 detectable that corresponded to the intensity peak of GFP-Atg8. 3xtagBFP-Pho8 was neither excluded from the vacuolephagophore contact site nor from the NVJ in both analyzed growing conditions (stationary and starved). This was in line with the observed distribution of GFP-Pho8 by Dawaliby et al. (2010) and suggested that the exclusion of Vph1-GFP was selective.

Toulmay and Prinz (2013) used the lipophilic styryl dye FM4-64 which specifically stains the vacuolar membrane as a marker for the formation of lipid domains. This dye was found to be excluded from domains in the vacuolar membrane in late stationary cells (2 days) similar to Vph1-GFP and colocalized with Vph1-GFP (Toulmay and Prinz, 2013). According to these findings, FM4-64 seemed to be a useful tool to further characterize the specialized membrane domain at the vacuolephagophore contact site.

Therefore, plasmid-encoded GFP-ATG8 and APE1 were expressed in an atg $8 \triangle$ strain as described before (chap. 4.2.1). Stationary cells were incubated with FM4-64 $(20 \mu \mathrm{g} / \mathrm{ml})$ for $30 \mathrm{~min}$. Afterwards they were either directly analyzed by fluorescence microscopy or after starvation for $2 \mathrm{~h}$ in SD-N medium (Figure 4.27).

FM4-64 was homogenously distributed at the vacuolar membrane. There were no visible exclusions at the vacuole-phagophore contact site nor at the NVJ which corresponded to Dawaliby et al. 2010, who observed that FM4-64 has access to the NVJ. The contact site of phagophore and vacuole as well as vacuole and nuclear ER seemed to be distinct from the formed lipid domains in late stationary cells (Toulmay and Prinz, 2013). However, measuring fluorescence profiles along the vacuole-phagophore contact site revealed slight decreases in the fluorescence 
intensity of FM4-64 that corresponded to the peaks of GFP-Atg8. All together these findings indicated that there might be a change in the lipid composition of the vacuole-phagophore contact site compared to the rest of the vacuolar membrane but did not prove the formation of a lipid raft. Further experiments are required to validate that special lipids are enriched at the contact site.

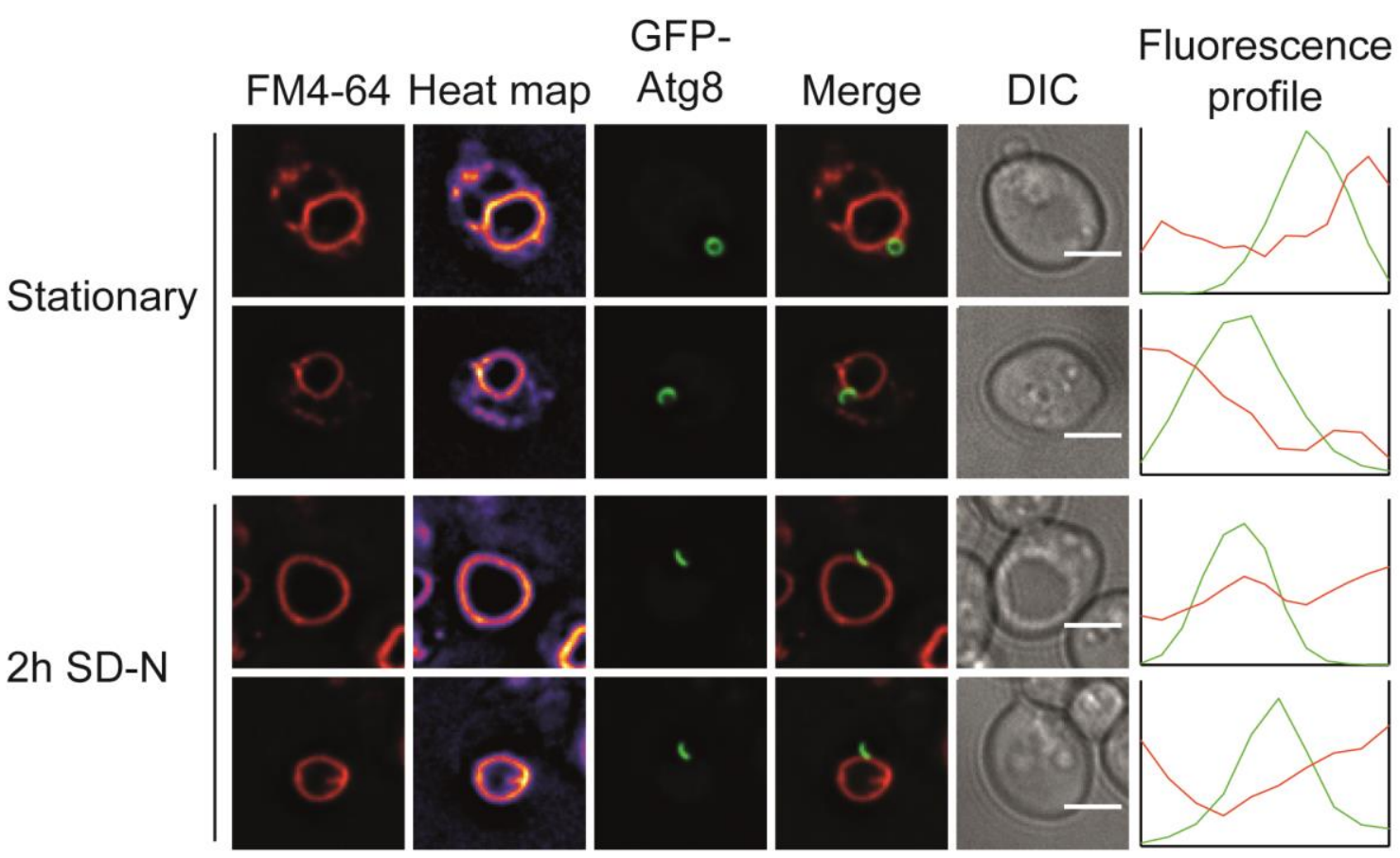

Figure 4.27: FM4-64 is not excluded from the contact site between vacuole and phagophore and there is no flux of FM4-64 to the phagophore

Analysis of the distribution of FM4-64 at the vacuolar membrane in APE1-overexpressing cells. In an atg $8 \triangle$ strain, plasmid-encoded GFP-ATG8 and APE1 were expressed using the ATG8 and the CUP1 promoter respectively. Cells were grown over night in selection medium with $100 \mu \mathrm{M} \mathrm{CuSO}{ }_{4}$ to stationary phase (OD600 5). FM4-64 $(20 \mu \mathrm{g} / \mathrm{ml})$ was added to the medium and cells were incubated for $30 \mathrm{~min}$. They were either directly analyzed by fluorescence microscopy or starved for $2 \mathrm{~h}$ in SD-N medium. For fluorescence microscopy the DeltaVision microscope, equipped with mCherry and GFP filter sets, was used. Scale bars represent $2 \mu \mathrm{m}$. Fluorescence profiles were measured along the contact site between the phagophore and the vacuole using Fiji (Schindelin et al., 2012). Corresponding graphs were plotted with the GFP (green) and FM4-64 (red) intensities on different axes using GraphPad Prism ${ }^{\circledR} 7$ (GraphPad Software). Heat maps were generated by coloring the FM464 channel according to its intensity with the look-up-table fire (bright (yellow) to dark (deep blue)) using Fiji.

In stationary cells, several punctuate structures were stained by FM4-64 in addition to its vacuolar localization. Since FM4-64 is transported to the vacuole via endocytosis (Vida and Emr, 1995), the observed punctuate structures were likely to be endosomes. By transferring the cells to SD-N medium extracellular FM4-64 was washed off stopping further transport of FM4-64 to the vacuole. Therefore, the dye was found to only stain the vacuolar membrane in starved cells. 
FM4-64 did not stain the phagophore indicating that there was no direct membrane contact between phagophore and vacuolar membrane which would lead to a flux of lipids from the vacuole to the phagophore. However, a transfer of lipids from the vacuolar membrane to the phagophore as it is described for other organellar contact sites is still possible, but not detectable using FM4-64.

\subsubsection{Atg21 is part of the vacuole-phagophore contact site}

To analyze whether the dot-like localization of Atg21-YFP corresponded to the identified contact site between vacuole and phagophore, the APE1-overexpression assay was used. The exclusion of Vph1 was used as a marker to visualize the vacuolephagophore contact site. To be able to image the phagophore, Atg21 and Vph1 simultaneously, VPH1 was chromosomally tagged with mCherry in an atg8 $\operatorname{atg} 21 \Delta$ strain. In the resulting strain, plasmid-encoded ATG21-YFP and mTagBFP2-ATG8 were expressed using their endogenous promoters. APE1 was overexpressed from the high copy pYEX-BX vector using the CUP1 promoter. Cells were cultivated as described for previous experiments (chap. 4.2.1) and starved for $1 \mathrm{~h}$ in SD-N medium before they were analyzed by fluorescence microscopy (Figure 4.28).

A

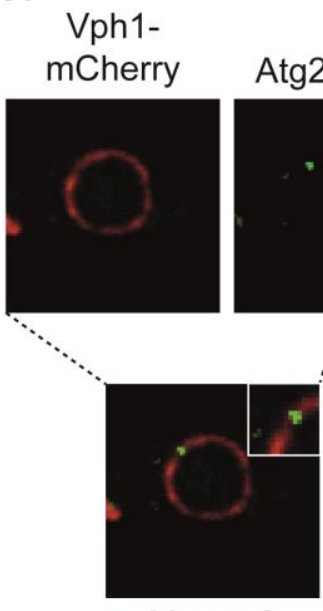

Merge 2
Atg21-YFP

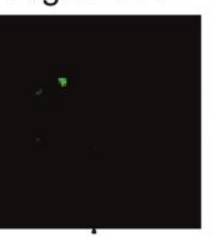

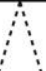

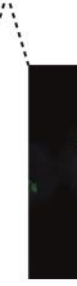

Merge 3
B mTagBFP2-

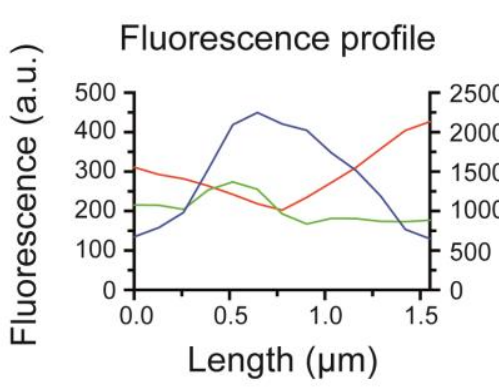

Figure 4.28: Atg21 localizes to the contact site between vacuole and phagophore

Analysis of the localization of Atg21-YFP at the phagophore in relation to the vacuole-phagophore contact site in APE1-overexpressing cells. In an atg8 atg21 $V P H 1$ - $m$ Cherry strain, plasmid-encoded mTagBFP2-ATG8 and ATG21-YFP were expressed using their endogenous promoters. APE1 was overexpressed from the high copy pYEX-BX vector using the CUP1 promoter. Cells were grown over night in selection medium with $100 \mu \mathrm{M} \mathrm{CuSO}_{4}$. Stationary cells (OD600 5) were starved for $1 \mathrm{~h}$ in SD-N medium and analyzed by fluorescence microscopy using the DeltaVision microscope equipped with DAPI, mCherry and GFP filter sets (A). The scale bar represents $2 \mu \mathrm{m}$. Fluorescence profiles (B) were measured along the contact site of the phagophore and the vacuole using Fiji (Schindelin et al., 2012). Corresponding graphs were plotted with the GFP (green) and mCherry (red) intensities on one axis and the mTagBFP2 intensity on the other using GraphPad Prism ${ }^{\circledR} 7$ (GraphPad Software). 
The fluorescence intensity of the mTagBFP2-Atg8 signal was very low resulting in the requirement for high-energy light and long exposure times in the DAPI channel. The high-energy light used for excitation in the DAPI channel further bleached the GFP and mCherry channels yielding in low fluorescence intensities in all imaged channels. Therefore, almost no mTagBFP2-Atg8 positive phagophores were observed in the analyzed cells. Figure 4.28 shows one example of a cell where all fluorescently-labeled proteins could be adequately imaged. However, due to the low fluorescence intensities the observed fluorescence signals should be evaluated carefully.

As observed before (chap. 4.2.2), Atg21-YFP localized to a single dot-like structure at the edge of the mTagBFP2-Atg8 positive phagophore which was in close proximity to the vacuolar membrane. Furthermore, Vph1-mCherry was found to be excluded from the corresponding region at the vacuolar membrane. This validated the formation of a contact between phagophore and vacuole. The measured fluorescence profiles along the vacuole-phagophore contact site revealed a correlation between the intensity peak of Atg21-YFP and the decrease in the signal intensity of Vph1-mCherry (Figure 4.28 B). Together with the earlier results indicating that Atg21 is a component of a Vac8-containing complex (chap. 4.2.5.4), this suggested that Atg21 was part of the contact site between vacuole and phagophore.

\subsubsection{The ER is in close proximity to the edges of the growing phagophore but not to the vacuole-phagophore contact site}

Besides its vicinity to the vacuole, the PAS is frequently reported to be adjacent to the ER (Suzuki et al., 2013; Graef et al., 2013; Gomez-Sanchez et al., 2018). Recently, an Atg2-dependent connection between the ER and the phagophore was identified that establishes an ER-phagophore contact site (Gomez-Sanchez et al., 2018; Kotani et al., 2018). Therefore, the question arose if there was a correlation between the ER-phagophore and the vacuole-phagophore contact site and whether both contact sites could coexist. The distribution of the phagophore relative to the ER and the vacuole was analyzed using the APE1-overexpression assay.

To visualize the ER, the ER resident protein Sec63 was C-terminally tagged with GFP while the vacuole was labeled by $3 x \operatorname{tagBFP}-\mathrm{Pho8}$. In the $\operatorname{atg} 8 \Delta 3 x \operatorname{tagBFP}-\mathrm{PHO} 8$ 
strain, plasmid-encoded SEC63-GFP and mCherry-ATG8 were expressed using their endogenous promoters. APE1 was overexpressed from the high copy pRS423 vector using the CUP1 promoter. Cells were grown as described before (chap. 4.2.1) and starved for $1 \mathrm{~h}$ in SD-N medium before they were analyzed by fluorescence microscopy (Figure 4.29).

As expected (Suzuki et al., 2013; Graef et al., 2013; Gomez-Sanchez et al., 2018), Sec63-GFP and therefore the ER was found to be in close proximity to the edges of the mCherry-Atg8 positive phagophore. However, Sec63-GFP was never observed to be in vicinity to the vacuole-phagophore contact site. This suggested that both contact sites were formed independent of each other and could therefore coexist. The interplay of both contact sites will need further investigation.

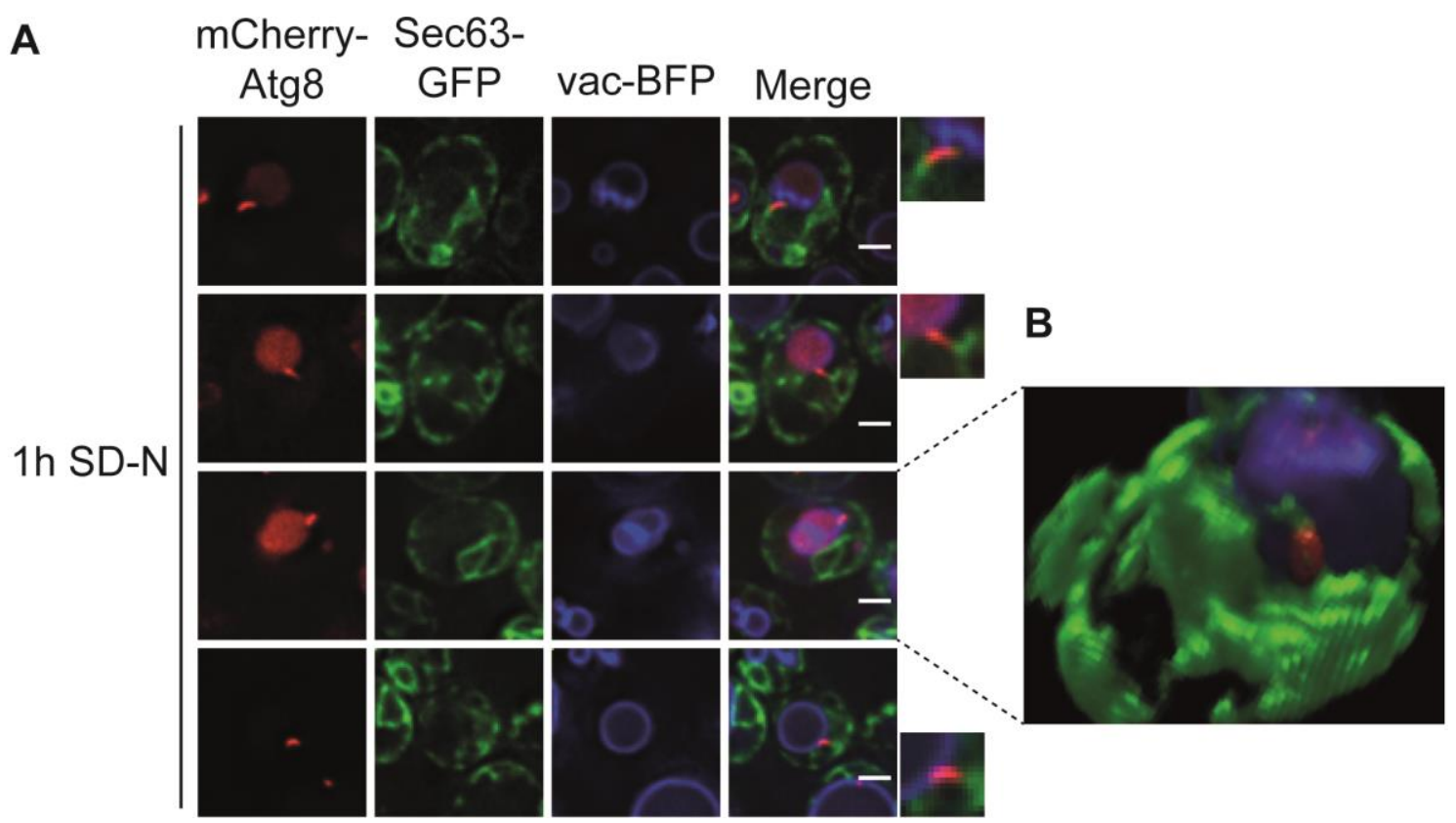

Figure 4.29: The ER is in close proximity to the edges of the growing phagophore but not to the vacuole-phagophore contact site

Analysis of the localization of Sec63-GFP in relation to the phagophore and the vacuole-phagophore contact site in APE1-overexpressing cells. In an atg8 3xtagBFP-PHO8 strain, plasmid-encoded SEC63-GFP and mCherry-ATG8 were expressed using their endogenous promoters. APE1 was overexpressed from the high copy pRS423 vector using the CUP1 promoter. Cells were grown over night in selection medium with $100 \mu \mathrm{M} \mathrm{CuSO}_{4}$. Stationary cells $\left(\mathrm{OD}_{600} 4\right)$ were starved for $1 \mathrm{~h}$ in SD-N medium and analyzed by fluorescence microscopy using the DeltaVision microscope equipped with DAPI, mCherry and GFP filter sets. Scale bars represent $2 \mu \mathrm{m}$. (B) 3D-projection of all focal planes of a cell using the freeware mode of the Huygens Professional X11 software (Scientific Volume Imaging). 


\subsection{Identification of unknown interaction partners of Atg21}

The third and last substantial goal of this thesis was the identification of unknown interaction partners of Atg21 to elucidate its further roles apart from its function in Atg8 lipidation.

Using gel filtration chromatography, Atg21 was identified as part of a high molecular weight complex. Without success, a variety of techniques, including recombinant pull down assays and GFP-TRAP approach followed by MS analysis, were applied to identify the interacting proteins of Atg21 in its high molecular weight complex (Juris, 2014). Since the high molecular weight complex of Atg21 was very unstable and sensitive to the applied buffer conditions, a method was required to identify protein-protein interactions in their native environment (Juris, 2014).

In the last 10 years a set of techniques has evolved which allow a proximity-based labeling of proteins in living cells. This in vivo-labeling approaches do not require the preservation of protein complexes during lysis and affinity purification since the covalently bound labels withstand harsh lysis and washing conditions. Thereby, these techniques are ideal to identify weak and transient interactions in their natural environment. A further advantage is their applicability to insoluble and membrane associated proteins (Kim and Roux, 2016). Therefore, one of these techniques, the proximity-dependent biotin identification (BioID) assay, was chosen to overcome the previous problems in the purification of Atg21-complexes and to identify unknown interaction partners of Atg21.

\subsubsection{Establishing and optimizing the BiolD assay}

The BioID assay takes advantage of the bifunctional DNA-binding biotin protein ligase from E. coli (BirA) (Roux et al., 2012). In its host organism BirA mediates the selective biotinylation of a subunit of the acetyl-CoA carboxylase. Furthermore, it transcriptionally regulates the biotin synthetic operon (Chapman-Smith and Cronan, 1999). Biotinylation is a two-step reaction where initially an activated biotin species (biotinoyl-5'-AMP) is generated by the combination of biotin and ATP (Lane et al., 1964). The activated biotin stays associated with the active site of BirA until it is covalently attached to a specific lysine residue within a small recognition sequence of the acetyl-CoA carboxylase (Chapman-Smith and Cronan, 1999). The 
mutation of arginine 118 to glycine (BirA*) significantly reduces the affinity of BirA* for biotinoyl-5'-AMP whereupon it is released to the cytosol. The free and highly reactive biotinoyl-5'-AMP will target-unspecific react with primary amines (e.g. lysine side chains) and thereby biotinylate surrounding proteins within a range of 10 nm (Choi-Rhee et al., 2004; Cronan, 2005; Kim et al., 2014).

Initially, the BioID assay was developed for the mammalian system but was recently adapted for $S$. cerevisiae by Opitz et al. (2017). Figure 4.30 shows the suggested workflow for the identification of proximal and interacting proteins of a target protein in yeast (Opitz et al., 2017).

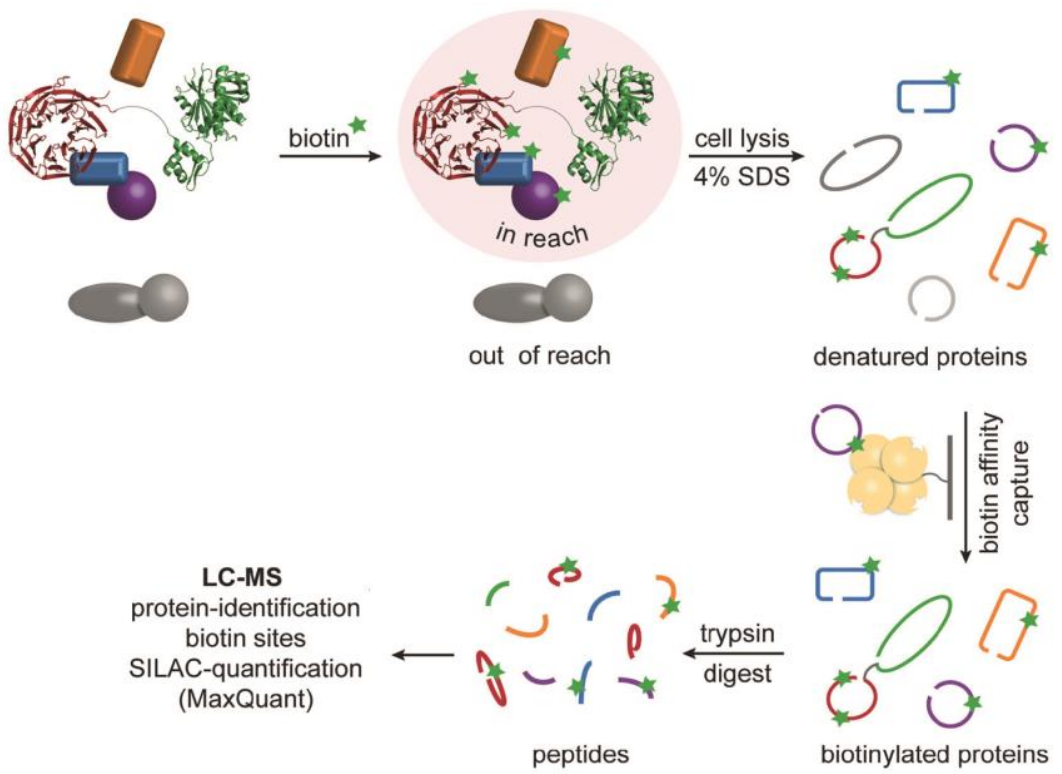

Figure 4.30: Workflow of the BioID assay (modified from Opitz et al. (2017))

The BioID assay is a technique for the in vivo and proximity-dependent biotinylation of proteins which can be affinity purified by streptavidin (Roux et al., 2012). A promiscuous mutant (BirA*, green) of the biotin protein ligase from $E$. coli is fused to a protein of interest (red) and expressed in cells. Within the cell BirA* uses exogenously added biotin (green asterisks) in combination with intracellular ATP to generate the highly reactive biotinoyl-5'-AMP. Due to its mutation BirA* releases biotinoyl-5'-AMP to the cytosol where it reacts with primary amines of lysine side chains of interacting (blue, purple) and proximal (orange) proteins of the protein of interest. Biotinylation takes place within a radius of approximately $10 \mathrm{~nm}$. Proteins that were labeled with biotin can be affinity captured from cell extracts under denaturing conditions (4\% SDS) using Strep-Tactin ${ }^{\circledR}$ columns (iba). After trypsin digestion, the peptides are subjected to LC-MS analysis for identification of proteins and biotin sites as well as SILAC-quantification.

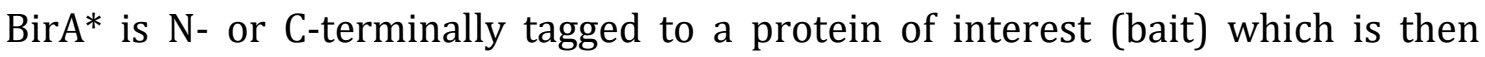
expressed in yeast cells. The cells are cultivated in the presence of $10 \mu \mathrm{M}$ biotin to induce the biotinylation of proteins in close proximity to the bait. Afterwards the cells are lysed under denaturing conditions (4\% (w/v) SDS) and biotinylated proteins are affinity captured using gravity flow Strep-Tactin ${ }^{\circledR}$ Sepharose ${ }^{\circledR}$ columns 
(iba). The captured proteins are eluted in the presence of $10 \mathrm{mM}$ biotin and subjected to SDS-PAGE. For the subsequent LC-MS analysis, the proteins are in-gel digested with trypsin and the resulting peptides are further purified and desalted. LC-MS analysis is performed to identify the captured proteins as well as their biotin sites. For the comparison of different strains or conditions, Opitz et al. (2017) recommend to combine the BioID assay with a SILAC-based approach. A detailed protocol of the described workflow was provided by Dr. Oliver Valerius and Dr. Kerstin Schmitt (Department of Molecular Microbiology and Genetics, GeorgAugust-University Göttingen). In the following this protocol was used to identify proximal and interacting proteins of Atg21.

\subsubsection{Construction of the BirA* fusion proteins}

The fundamental component of the BioID assay is the BirA* fusion protein. For the fusion of a Myc tagged BirA* to a target protein, initially BirA* fusion plasmids were generated for the integration of target genes in front of or behind the Myc-BirA* gene. As a backbone, the pUG35 and pUG36 vectors were used that were originally designed for the expression of yeGFP fusions under the control of the MET25 promoter. In these vectors the yeGFP was replaced by the Myc-BirA* gene thereby placing it five prime (pUG36-Myc-BirA*-MCS) or three prime (pUG35-MCS-Myc-

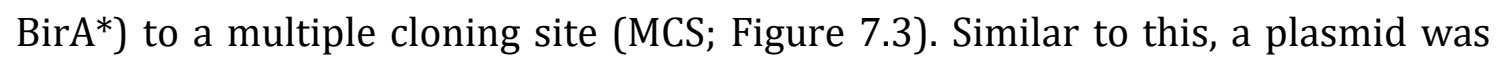
constructed expressing only Myc-BirA* (pUG36-Myc-BirA*) which was needed as a control for the BioID assay.

For cloning, the Myc-BirA* (hereafter named BirA*) was amplified by PCR using the plasmid pFA6a-Myc-BirA*_TRP1 as a template which was kindly provided by Dr. Sabrina Beckmann and Dr. Hans Dieter Schmitt (Department of Neurobiology; Max Planck Institute for Biophysical Chemistry). For the detailed cloning strategy see chap. 3.3.10. The Myc-tag was incorporated into the plasmids for a convenient Western-Blot analysis of the expression of the fusion proteins.

For the fusion with BirA*, ATG21 was cloned into the MCS of pUG36-Myc-BirA*-MCS and pUG35-MCS-Myc-BirA* (chap. 3.3.10). The resulting plasmids were used to express BirA*-ATG21 and ATG21-BirA* in respective $\operatorname{atg} 21 \Delta$ strains for the isolation of specifically biotinylated proteins. 


\subsubsection{BirA* fusion proteins complement the atg21 1 pApe1 maturation phenotype}

Prior to any BioID experiment the expression and function of the BirA* fusion proteins was validated. BirA*-ATG21, ATG21-BirA* or BirA* alone were expressed in an $\operatorname{atg} 21 \Delta$ strain. To induce different expression levels of the fusion proteins, the cells were grown in selection medium supplemented with varying concentrations of L-methionine (without, $0.3 \mathrm{mM}$ and $0.78 \mathrm{mM}$ ). Stationary cells (OD600 4-5) were alkaline lysed and resulting cell extracts were analyzed by Western-Blot (Figure $4.31 \mathrm{~A})$.

All three proteins (BirA* and both fusion proteins) could be detected in WesternBlot using the anti-Myc antibody which validated their successful expression. As expected, the protein levels varied depending on the concentration of L-methionine in the medium. Higher L-methionine concentrations $(0.78 \mathrm{mM}$ and $0.3 \mathrm{mM})$ downregulated the expression from the MET25 promoter resulting in low protein levels in the cell. The highest protein level of the BirA* fusion proteins and BirA* alone was observed in selection medium without any L-methionine. Ideally, BirA* fusion proteins should be expressed at a similar level to the respective WT protein. Since there was no Atg21 specific antibody available, expression levels could not be compared to the WT level.

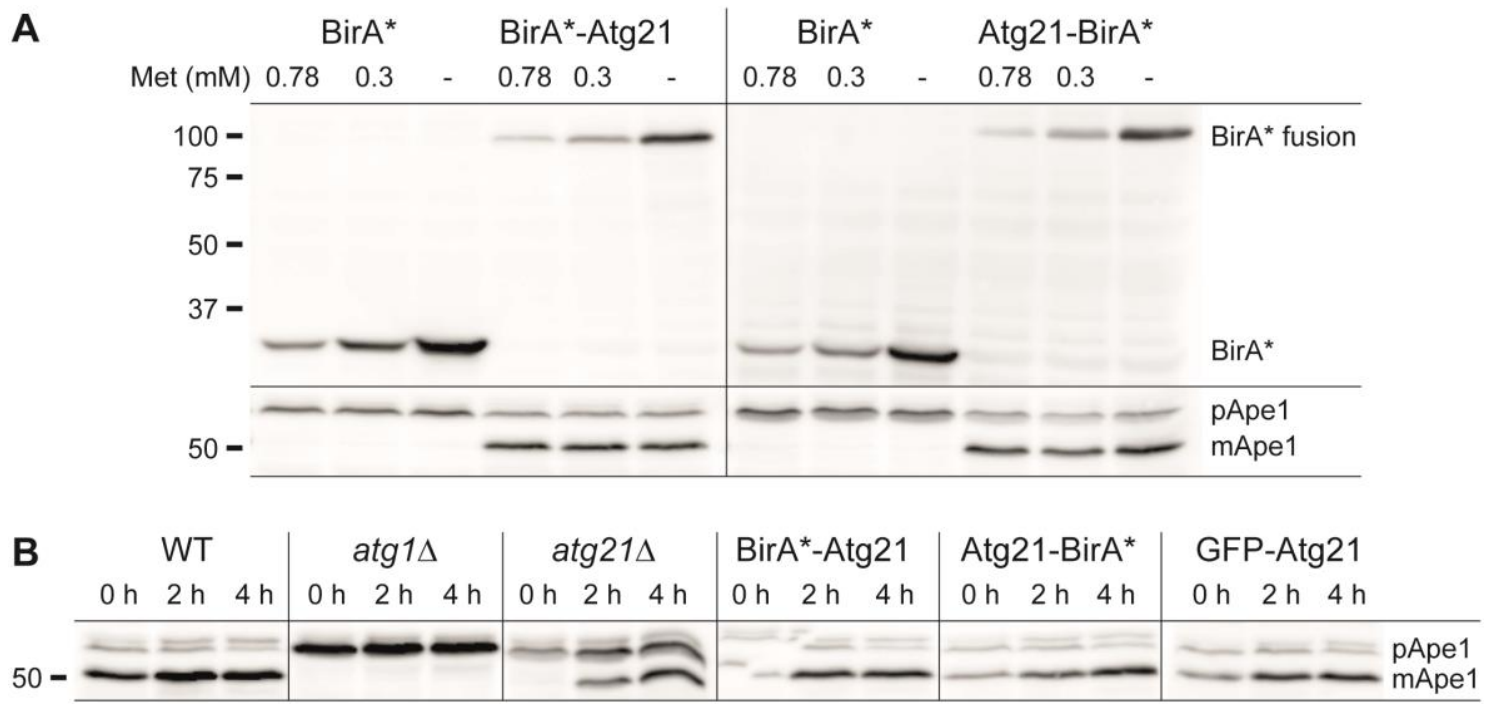

Figure 4.31: Expression levels and function of the BirA* fusion proteins

Western-Blot analysis of the expression of the BirA* fusions of Atg21 and their ability to complement the phenotype of $\operatorname{atg} 21 \Delta$ in the Cvt-pathway. (A) In an atg21 $\Delta$ strain, plasmid-encoded BirA*-ATG21, ATG21-BirA* and BirA* alone were expressed using the MET25 promoter. Cells were grown over night in selection medium without L-methionine (Met; -) or supplemented with different 
concentrations of it ( $0.78 \mathrm{mM}, 0.3 \mathrm{mM}$ ). Stationary cells (OD600 4-5) were alkaline lysed and analyzed by Western-Blot. (B) Plasmid-encoded BirA*-ATG21, ATG21-BirA* and GFP-ATG21 were expressed

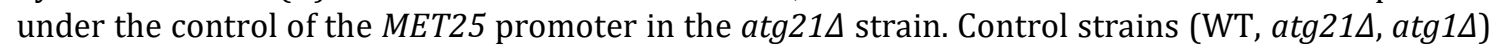
expressed BirA* alone. Cells were grown over night in selection medium without L-methionine to stationary phase (OD $6002-3)$, alkaline lysed and analyzed by Western-Blot for the maturation of pApe1. The molecular weight marker is depicted in $\mathrm{kDa}$.

Independent of the protein levels of BirA*-Atg21 and Atg21-BirA* in the cell, pApe1 was found to be efficiently matured while its maturation was blocked in presence of BirA* alone (Figure 4.31 A). In all following experiments, conditions were used which favor a high protein level of BirA*-Atg21 and Atg21-BirA* in the cell. Therefore, yeast strains were grown in selection medium without L-methionine. With this approach, the specific biotinylation of interacting and proximal proteins of Atg21 should be increased. However, overproduction of the BirA* fusion protein could increase its mislocalization and therefore the identification of false positive proteins due to unspecific biotinylation (Varnaitè and MacNeill, 2016). This had to be considered for all performed BioID experiments.

The extent of the restored pApe1 maturation was further assessed in comparison to BirA* expressing control strains (WT, $\operatorname{atg} 1 \Delta, \operatorname{atg} 21 \Delta$ ) as well as an GFP-ATG21 carrying strain (Figure $4.31 \mathrm{~B}$ ). The cells were grown in selection medium without L-methionine to stationary phase and starved in SD-N medium. The pApe1 maturation was analyzed after 0,2 and $4 \mathrm{~h}$ of starvation by Western-Blot analysis. Both BirA* fusion proteins restored the function of the Cvt-pathway $(0 \mathrm{~h})$ comparable to the WT strain and to the GFP-Atg21 carrying strain. They were therefore assumed to be functional. In the following, BirA*-Atg21 and Atg21-BirA* were both used to perform initial BioID experiments (chap. 4.3.1.3).

\subsubsection{Adapting the workflow of the BiolD assay for the identification of Atg21-interacting proteins}

To evaluate the ability of BirA*-Atg21 and Atg21-BirA* to specifically biotinylate surrounding proteins of Atg21, initial BioID experiments were performed according to the protocol of Opitz et al. (2017) (Figure 4.30; chap. 3.9.6). Atg16 is a well characterized interaction partner of Atg21 and was therefore used to analyze whether interacting proteins are biotinylated in the presence of BirA*-Atg21 or Atg21-BirA* and subsequently captured by the Strep-Tactin ${ }^{\circledR}$ columns. 
ATG16-HA was expressed in combination with either BirA*-ATG21, ATG21-BirA* or BirA* alone in an $\operatorname{atg} 21 \Delta \operatorname{atg} 16 \Delta$ strain. The cells were grown over night in selection medium without L-methionine to strongly induce the expression of the BirA* fusion proteins. To assess the effect of biotin on the biotinylation efficiency of BirA* either no biotin or $10 \mu \mathrm{M}$ biotin was added to the medium. Early stationary cells (OD 6002 ) were mechanically lysed using glass beads and biotinylated proteins were captured using $0.2 \mathrm{ml}$ gravity flow Strep-Tactin ${ }^{\circledR}$ Sepharose ${ }^{\circledR}$ columns (iba) (chap. 3.9.6). As suggested by Opitz et al. (2017), the eluted proteins were concentrated but using $10 \%(\mathrm{w} / \mathrm{v})$ TCA instead of a chloroform-methanol extraction. Input, elution and concentrated elution fractions were analyzed by Western-Blot analysis (Figure $4.32 \mathrm{~A})$. For the detection of biotinylated proteins, the PVDF membrane was blocked with a $3 \%(\mathrm{w} / \mathrm{v})$ bovine serum albumin (BSA) solution. The BSA solution was used instead of the skim milk containing blocking buffer to avoid false positive signals from biotinylated milk proteins. Afterwards the BSA-blocked PVDF membrane was decorated with a Strep-Tactin-HRP conjugate to detect biotinylated proteins.

Atg16-HA was found to be clearly enriched in the concentrated elution fraction of the BirA*-Atg21 as well as the Atg21-BirA* carrying strain compared to the strain expressing BirA* alone (Figure $4.32 \mathrm{~A}$ and Figure 7.4). Only minor amounts of Atg16-HA were observed to be unspecifically biotinylated by free BirA*. This indicated that Atg16-HA was specifically biotinylated by BirA*-Atg21 as well as Atg21-BirA* due to its interaction with Atg21 and corresponded to the findings of chap. 4.1.2.4. Furthermore, this supported the findings of chap. 4.3.1.2 that the function of Atg21 was not affected by its fusion to BirA*.

Interestingly, Atg16-HA was not found in the elution fraction but was detectable after the TCA precipitation indicating that the concentration of biotinylated proteins was very low in the relatively big elution volume. This was also observed for the directly detected biotinylated proteins and suggested that the precipitation of the elution fraction was indispensable for an adequate analysis of the captured proteins. Furthermore, the addition of biotin to the medium was essential for an efficient BirA*-dependent biotinylation of proteins. Almost no biotinylated proteins could be isolated from strains that were grown without additional biotin (Figure 4.32 A). Without the addition of biotin, 6 prominent protein bands were detected by the 
Strep-Tactin-HRP conjugate within the precipitated elution fractions of the analyzed strains. The band pattern was identical in the elution fractions of the BirA* as well as the BirA*-Atg21 carrying strain which suggested that the captured proteins might be also identical. These proteins were so prominent that they were even detectable in the non-concentrated elution fraction as well as in a CBB stained SDSpolyacrylamide gel (Figure 4.32 B). Since these bands were present in all analyzed strains, they were assumed to be naturally biotinylated proteins co-purified by the Strep-Tactin columns. At least 6 proteins are known to be naturally biotinylated in S. cerevisiae (Pirner and Stolz, 2006) which corresponded to the observed protein band pattern.

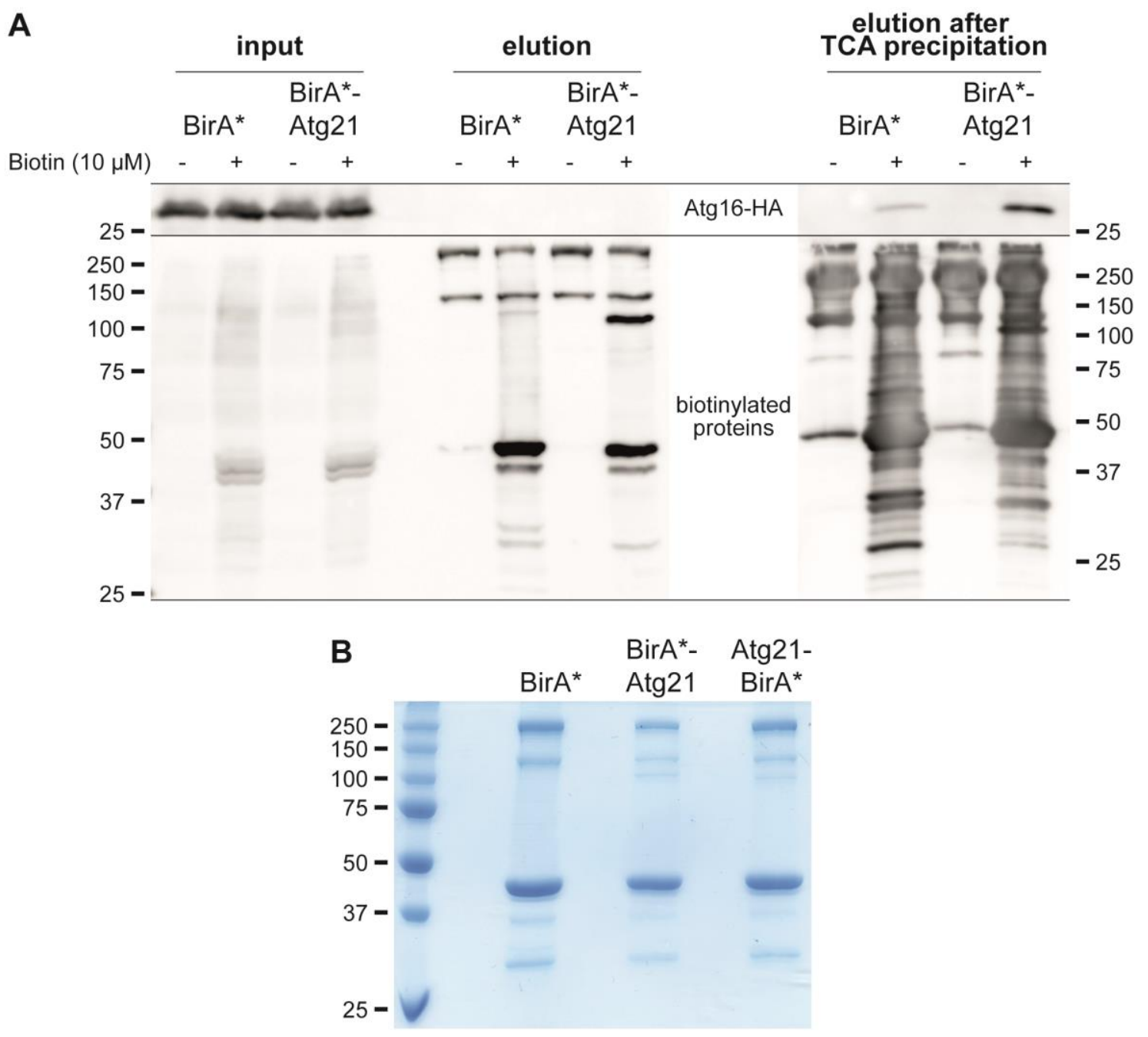

Figure 4.32: Pattern of the isolated biotinylated proteins from cells carrying BirA*-Atg21, Atg21-BirA* or BirA* alone

In an $\operatorname{atg} 21 \Delta \operatorname{atg} 16 \Delta$ strain, plasmid-encoded $A T G 16-H A$ was expressed in combination with BirA*ATG21, ATG21-BirA* or free BirA*. The expression was controlled by the CUP1 and MET25 promoter respectively. Cells were grown over night in selection medium without L-methionine in absence or presence of $10 \mu \mathrm{M}$ biotin. Early stationary cells $\left(\mathrm{OD}_{600} 2\right)$ were processed according to the BioID workflow. Biotinylated proteins were isolated using $0.2 \mathrm{ml}$ gravity flow Strep-Tactin ${ }^{\circledR}$ Sepharose ${ }^{\circledR}$ 
columns (iba) and precipitated in presence of $10 \%(\mathrm{w} / \mathrm{v}$ ) TCA. Input, elution and precipitated elution fractions of the BirA*-Atg21 and BirA* alone expressing cells were analyzed by Western-Blot (A). For the detection of biotinylated proteins, the PVDF membrane was blocked in 3\% (w/v) BSA in TBST and decorated with a Strep-Tactin-HRP conjugate. In addition, biotinylated proteins from the precipitated elution fractions from all three strains grown in presence of $10 \mu \mathrm{M}$ biotin were CBB stained in an SDS-polyacrylamide gel (B). The molecular weight marker is depicted in kDa.

Upon addition of biotin to the medium, a very complex pattern of biotinylated proteins could be detected by the Strep-Tactin-HRP conjugate in the precipitated elution fractions of all analyzed strains (Figure 4.32 A and Figure 7.4). Since these proteins were not detected in the absence of additional biotin, they seemed to be BirA*-dependent biotinylated proteins. The pattern of biotinylated proteins slightly differed between the precipitated elution fractions of the analyzed strains suggesting that the free BirA* labeled different proteins compared to BirA*-Atg21 or Atg21-BirA*. In the presence of biotin, another very prominent band appeared in addition to the naturally biotinylated proteins. This band was clearly distinguishable from the smear-like band pattern of biotinylated proteins and was even detectable in the non-concentrated elution fraction (Figure $4.32 \mathrm{~A}$ arrows). With a molecular weight of approximately $35 \mathrm{kDa}$ (in the BirA* sample) or $100 \mathrm{kDa}$ (in the BirA*-Atg21 sample) this band likely represented the BirA* or BirA*-Atg21 protein which were captured by the Strep-Tactin columns due to self-biotinylation.

Apart from the naturally biotinylated proteins and the BirA* fusion proteins themselves, none of the BirA*-dependent biotinylated proteins could be stained by CBB in a SDS-polyacrylamide gel (Figure 4.32 B). Therefore, it was not possible to directly analyze distinct bands by MS to identify potential interaction partners of Atg21 although an attempt was made (data not shown). To solve this problem a SILAC-based BioID approach was considered as it was described by Opitz et al. (2017).

\subsubsection{Quantitative Analysis of the biotinylated proteins using SILAC}

Western-Blot analysis and CBB staining demonstrated that even after TCA precipitation the amount of BirA*-dependent biotinylated proteins compared to the intrinsic biotinylated proteins was relatively low. It was almost impossible to reliably identify potential interaction partners of Atg21 by MS analysis alone (data not shown). This indicated the requirement of a quantitative method that allowed a targeted identification of those proteins that were specifically biotinylated by BirA*- 
Atg21. Therefore, the BioID assay was combined with the SILAC approach as it was suggested by Opitz et al. (2017).

The SILAC approach makes use of stable isotope labeled amino acids (e.g. by ${ }^{13} \mathrm{C}$ ) that are incorporated into proteins during protein syntheses (Ong and Mann, 2006). Thereby, up to three populations of cells which are grown in the presence of distinct combinations of the labeled amino acids (light, medium and heavy) can be compared in one batch. After combination of light, medium and heavy samples they remain distinguishable due to the mass shift that is introduced by the heavy amino acids and detectable by MS (Ong and Mann, 2006).

In this study, the SILAC approach was used to compare the biotinylated proteins captured from a BirA*-Atg21 carrying strain with those of two control strains as indicated in Figure 4.33. The labeling was performed using stable isotopes of arginine and lysine. To enable the efficient incorporation of the heavy amino acids, an arginine and lysine auxotrophic strain was generated by the deletion of the $A R G 4$ and LYS1 genes in the $\operatorname{atg} 21 \Delta$ strain. In the resulting $\operatorname{atg} 21 \Delta \arg 4 \Delta \operatorname{lys} 1 \Delta$ strain BirA*-Atg21 (light) or BirA* alone (medium) were plasmid-expressed using the MET25 promoter. BirA* alone was expressed to assess biotinylations which are generated by BirA* independent of the localization of Atg21. As a second control the

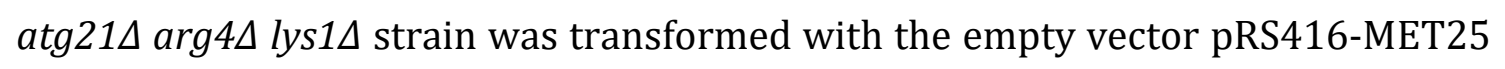
(heavy) to reveal naturally biotinylated proteins. Together both controls are also able to exclude those proteins that unspecifically bind to the Strep-Tactin ${ }^{\circledR}$ column. The light medium was supplemented with L-arginine and L-lysine with a natural isotope abundance while the combinations of ${ }^{13} \mathrm{C}_{6}$ - $\mathrm{L}$-arginine with $4,4,5,5$ - $\mathrm{D}_{4}$ - $\mathrm{L}$ lysine (medium) or ${ }^{13} \mathrm{C}_{6}{ }^{15} \mathrm{~N}_{4}$-L-arginine with ${ }^{13} \mathrm{C}_{6}{ }^{15} \mathrm{~N}_{2}$-L-lysine (heavy) were added to the respective heavier media. The media were further supplemented with $10 \mu \mathrm{M}$ biotin to enable an efficient biotinylation. After the individual cultivation and labeling of all three strains, they were pooled in a 1:1:1 ratio and further processed according to the SILAC-based BioID workflow (Figure 4.33). Biotinylated proteins were isolated using a $1 \mathrm{ml}$ gravity flow Strep-Tactin ${ }^{\circledR}$ Sepharose ${ }^{\circledR}$ column (iba). After TCA precipitation, the whole elution fraction was separated on a SDSpolyacrylamide gel and subjected to $\mathrm{CBB}$ staining. For the in-gel digestion with trypsin, the sample lane (length approx. $3 \mathrm{~cm}$ ) was divided into 10 sections as 
indicated in Figure 4.33. Each section was afterwards analyzed as an individual sample by LC-MS. Trypsin digestion as well as the subsequent purification and desalting of the resulting peptides was kindly performed by Olaf Bernhard (Department of Cellular Biochemistry, University Medical Center Göttingen).

LC-MS and the subsequent data analysis was performed by Dr. Oliver Valerius and Dr. Kerstin Schmitt (Department of Molecular Microbiology and Genetics, GeorgAugust-University Göttingen) using the $Q$ exactive ${ }^{\mathrm{TM}}$ HF Hybrid QuadrupolOrbitrap ${ }^{\mathrm{TM}}$ mass spectrometer (Thermo Fisher Scientific). Identification of proteins and their biotinylated lysine residues as well as the SILAC quantifications were performed using the Proteome Discoverer ${ }^{\mathrm{TM}} 2.2$ software (Thermo Fisher Scientific). The SequestHT and Mascot search algorithms were used for the database search against a S. cerevisiae specific database (chap. 3.10). The database search resulted in quantitative SILAC protein ratios which described the relative abundance of a protein in one sample compared to the other (e.g. light/medium).
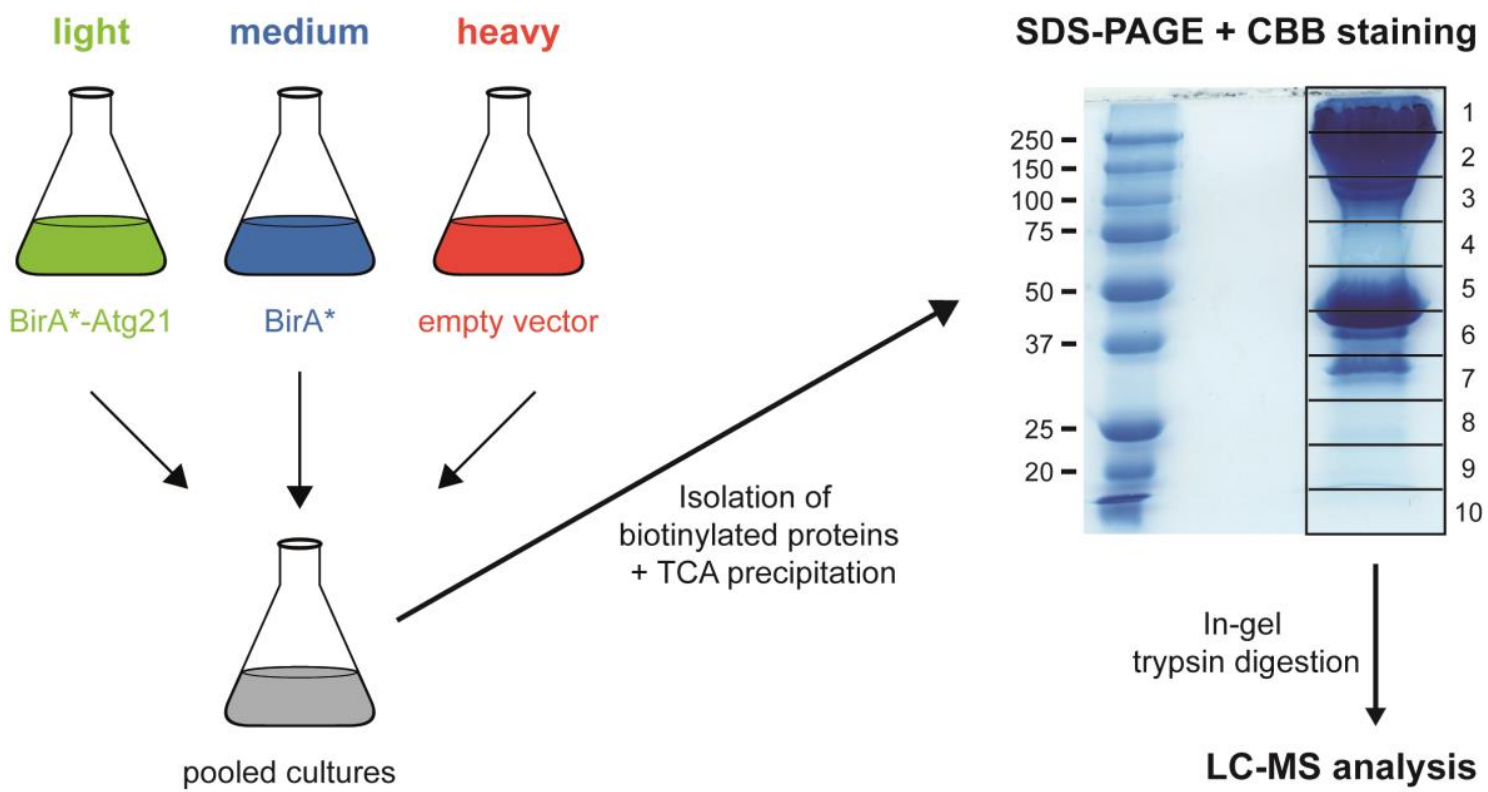

\section{Figure 4.33: Workflow for the SILAC-based BioID experiments}

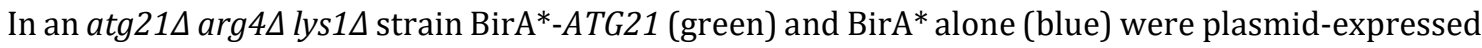

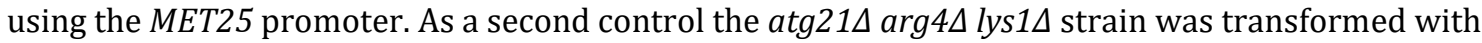
the empty vector pRS416-MET25 (red). The cells were individually cultured in selection medium without L-methionine supplemented with $10 \mu \mathrm{M}$ biotin and with combinations of $50 \mathrm{mg} / \mathrm{ml}$ of stable isotope labeled L-arginine and L-lysine as indicated ${ }^{13} \mathrm{C}_{6}$ - $\mathrm{L}$-arginine with $4,4,5,5$ - $\mathrm{D}_{4}$-L-lysine for medium and ${ }^{13} \mathrm{C}_{6}{ }^{15} \mathrm{~N}_{4}$-L-arginine with ${ }^{13} \mathrm{C}_{6}{ }^{15} \mathrm{~N}_{2}$-L-lysine for heavy). Equal amounts of stationary cells $\left(\mathrm{OD}_{600} 2\right)$ of each strain were pooled and biotinylated proteins were isolated using a $1 \mathrm{ml}$ gravity flow Strep-Tactin ${ }^{\circledR}$ Sepharose ${ }^{\circledR}$ column (iba). After TCA precipitation, the whole elution fraction was separated on a SDS-polyacrylamide gel and subjected to CBB staining. For the in-gel digestion with trypsin, the sample lane (length approx. $3 \mathrm{~cm}$ ) was divided into 10 sections. Each section was analyzed as an individual sample by LC-MS. The molecular weight marker is depicted in kDa. 
The database search initially identified 1076 proteins which were captured by the Strep-Tactin ${ }^{\circledR}$ column. To select for those proteins that were especially enriched in the BirA*-Atg21 derived light sample, the SILAC protein ratios of light/medium and light/heavy were filtered for ratios $\geq 1.5$. In addition, the proteins were filtered for those identified by at least 2 unique peptides and that showed a high confidence as well as at least 3 abundance counts in the light sample. Contaminations, e.g. keratin, were excluded by filtering for S. cerevisiae specific proteins. Finally, the filtering resulted in the identification of 18 proteins that were specifically enriched in the light sample.

The 18 identified proteins were ordered according to their number of peptide spectrum matches (PSM) which is the total number of identified peptide spectra matched for the protein. The PSM can be used to evaluate the abundance of an identified protein in the sample whereby high PSMs correspond to a high abundance. Remarkably, Atg21 itself was found as first hit in the list of the specifically enriched proteins of the light sample. It displayed the highest PSM (547) as well as high light/medium (26.156) and light/heavy (5.161) ratios suggesting a high abundance of Atg21 in the light sample. Furthermore, six distinct biotin sites were identified for Atg21. As the light sample derived from the BirA*-ATG21 expressing strain these findings highly indicated that the assay was working. Therefore, the remaining 17 identified proteins could be considered as potential interaction partners or at least components of Atg21-containing complexes.

Unexpectedly, none of the known interactors of Atg21, Atg8 and Atg16, were identified among the Strep-Tactin captured proteins. Atg16 was already shown to be specifically biotinylated by BirA*-Atg21 (chap. 4.3.1.3), but is similar to Atg8 a relatively small protein (Atg8: $13.6 \mathrm{kDa}$; Atg16: $17.2 \mathrm{kDa}$ ) with an unfavorable distribution of trypsin sites. Therefore, it is reasonable to assume that in this approach they were not identifiable by MS analysis.

The second and also one of the most promising hits among the 17 identified proteins was Ykt6. It displayed a high PSM (166) as well as good light/medium (1.824) and light/heavy (1.601) ratios. Furthermore, two biotin sites could be identified in Ykt6. Interestingly, it was recently reported as the autophagosomal SNARE protein in autophagosome-vacuole fusion (Bas et al., 2018; Gao et al., 2018b). Further 
experiments are required to confirm the interaction of Atg21 with Ykt6. This interaction, however, could suggest a functional link between Atg21 and the autophagosome-vacuole fusion.

To further characterize the function of the 17 potential interaction partners of Atg21 they were grouped according to their reported association with cellular compartments (Figure 7.5). The spatial grouping of the potential interactors of Atg21 was performed with the Gene Ontology Slim Mapper of the Saccharomyces Genome Database. Remarkably, about $80 \%$ of the identified candidates were found to be membrane associated (Figure 7.5). They were mainly associated with the vacuolar membrane $(\approx 75 \%)$ or with membranes of the endomembrane system $(\approx 50 \%)$ as well as cytosolic vesicles $(\approx 35 \%)$. This perfectly correlated with the reported localization of Atg21 to PI3P and $\mathrm{PI} 3,5 \mathrm{P}_{2}$ positive membranes at endosomes, the vacuole or the PAS and further validated the identified proteins as potential interaction partners of Atg21 (Stromhaug et al., 2004; Meiling-Wesse et al., 2004; Krick et al., 2008a).

Functionally more than half of the potential interaction partners of Atg21 ( 9 out of 17) participated in cellular transport processes. Among these proteins, 6 were designated to play a role in vesicle-mediated transport such as endosomal or Golgivesicle transport. They were therefore promising candidates for endosomal or vacuolar interactors of Atg21 and can probably provide a hint for its non-autophagic function. A detailed characterization of the potential interaction partners of Atg21 is still in progress. To verify the results from the first experiment and to allow a statistical evaluation of the determined quantitative SILAC ratios, three more biological replicates of the above described experiment were performed in this study. All three biological replicates were processed in parallel according to the described SILAC-based BioID workflow (Figure 4.33) and subjected to LC-MS analysis. The initial database search using the Proteome Discoverer ${ }^{\mathrm{TM}} 2.2$ software (Thermo Fisher Scientific) identified more than 1900 proteins. Filtering of the obtained data set according to the above described parameters resulted in a list of 111 identified proteins that were enriched in at least one of the light samples. A more comprehensive analysis of the complex dataset which will also include the first performed SILAC-based BioID experiment as well as statistical evaluations will be 
performed ongoing from this study using the MaxQuant and Perseus software (Cox and Mann, 2008). This will help to reliably determine potential interaction partners of Atg21. Nevertheless, the 17 identified potential interaction partners of Atg21 which were identified by the first performed SILAC-based BioID experiment provide a promising basis for the elucidation of the function of Atg21. Furthermore, these findings supported that the BioID assay is a powerful tool to analyze the composition of target protein-containing complexes. 


\section{Discussion}

Autophagy is a degradative membrane trafficking pathway that transports cellular components to the vacuole in yeast or the lysosome in mammals to maintain cellular homeostasis. Targets for degradation are a variety of cytosolic components including protein aggregates, damaged or superfluous organelles or invading pathogens. The transport process can either be unselective or selective but always involves the formation of a double-membraned vesicle, the autophagosome, which encloses the cargo. Autophagosome biogenesis is initiated at the pre-autophagosomal structure (PAS) where a cup-shaped membrane structure is assembled de novo (phagophore) to engulf cytosolic material. The closed autophagosome fuses with the vacuole to release the autophagic body, consisting of the cargo enclosed by the inner autophagic membrane, to the vacuolar lumen. The autophagic body is lysed making its content accessible for degradation and reuse (Wen and Klionsky, 2016).

A crucial factor for autophagosome biogenesis is the generation of PI3P at the PAS (Kihara et al., 2001). The presence of PI3P on autophagic membranes is deciphered by $\beta$-propellers that bind polyphosphoinositides (PROPPIN). PROPPINs are a highly conserved family of WD40-repeat proteins which fold as seven-bladed $\beta$-propellers. PI3P binding is mediated by a conserved FRRG-motif that participates in the formation of two lipid-binding sites at the circumference of the propeller (Krick et al., 2012; Baskaran et al., 2012; Watanabe et al., 2012; Scacioc et al., 2017).

This study focused on the investigation of the molecular functions of the $S$. cerevisiae PROPPIN Atg21 including its role in the lipidation of the ubiquitin-like protein Atg8 (Juris et al., 2015). Unlike its homolog Atg18, Atg21 is not essential for bulk autophagy but is indispensable for selective types of autophagy like the Cvt-pathway (Barth et al., 2001; Barth et al., 2002; Stromhaug et al., 2004; Meiling-Wesse et al., 2004). Although selective types of autophagy require special factors, like selective cargo receptors, they share most of the core autophagic machinery involved in unselective autophagy (Farré and Subramani, 2016). Therefore, molecular mechanisms uncovered in selective autophagy are often relevant and transferrable to other forms of autophagy. Furthermore, most of the core autophagic components, 
including the PROPPIN-family, exist in the genome of all eukaryotic organisms validating their evolutionary conservation. The knowledge of the autophagic processes in S. cerevisiae is therefore also relevant for higher eukaryotes including the investigation of human diseases.

\subsection{Structural and dynamical organization of the Atg8 lipidation complex}

A study on the function of Atg21 during autophagy and the Cvt-pathway revealed that it organizes the conjugation of Atg8 to PE at the autophagic membrane. During this process, it directly interacts with the Atg8 Atg3 conjugate via the conserved F5K6-motif of Atg8. Furthermore, Atg21 recruits the Atg12 Atg5/Atg16 complex via the interaction with Atg16 and thereby arranges the E3-like Atg12 Atg5 conjugate within reach of its substrate the E2-like enzyme Atg3. By binding to PI3P at the autophagic membrane, Atg21 further positions the Atg8 lipidation complex in close proximity to PE (Figure 2.13; Juris et al., 2015).

Although phylogenetic analyses revealed that there is no orthologue of Atg21 in higher eukaryotes, its function in the lipidation of Atg8 seems to be conserved from yeast to human (Polson et al., 2010; Dooley et al., 2014; Juris et al., 2015). The human PROPPIN WIPI2b, an isoform of WIPI2, facilitates the conjugation of LC3 to PE by directly interacting with Atg16L1 a human orthologue of Atg16. WIPI2b recruits the Atg12 Atg5/Atg16L1 complex to the PI3P-positive omegasome and thereby defines the site of LC3 lipidation (Dooley et al., 2014). Likewise, the PROPPIN Atg18a from Schizosaccharomyces pombe (S. pombe) is required for the targeting of Atg8 and the Atg12 Atg5/Atg16 complex to the PAS. Furthermore, S. pombe Atg18a interacts with the Atg12 Atg5/Atg16 complex indicating that it may also promote Atg8 lipidation at the autophagic membrane (Sun et al., 2013). These findings imply that the overall function of Atg12 Atg5/Atg16 complex recruitment is a conserved feature of members of the PROPPIN-family. 


\subsubsection{Molecular details of the interacting domains of Atg16 and Atg21 and model of the overall structure of the Atg8 lipidation complex}

In parallel to this study, the cooperation partners Dr. Karin Kühnel and Dr. Janina Metje (former members of the Department of Neurobiology, Max Planck Institute for Biophysical Chemistry, Göttingen) were able to crystallize KlAtg21 in complex with the CCD of AgAtg16 (residues 40-124). The obtained crystals diffracted up to a resolution of $4 \AA$ and the structure was solved by molecular replacement with the known structures of PaAtg18 and ScAtg16 (Figure 4.1; Metje, 2017). This is the first available structure of an Atg21 orthologue, but even more important the first structure of a PROPPIN-family member in complex with its interactor. So far, only the structures of KlHsv2, KmHsv2 and PaAtg18 had been solved (Krick et al., 2012; Baskaran et al., 2012; Watanabe et al., 2012; Scacioc et al., 2017).

A variety of WD40-repeat proteins have been crystallized in complex with their interacting proteins, nevertheless the KlAtg21-AgAtg16 complex is the first crystal structure of a PROPPIN complex. A reason for this might be that so far only little is known about the interactors of the PROPPIN-family. Zheng et al. (2017) performed a $3 \mathrm{D}$ reconstruction of the complex of human $A \operatorname{tg} 2 \mathrm{~B}$, an orthologue of $A \operatorname{tg} 2$, and the PROPPIN WIPI4 using single-particle electron microscopy. The resulting structure with a resolution of about $18 \AA$ revealed a club-shaped form of the complex but could not provide molecular details on the interaction of Atg2B and WIPI4 (Zheng et al., 2017). Therefore, the crystal structure of KlAtg21 in complex with the CCD of AgAtg16 remains the only structure that provides detailed information on the binding mechanism of a PROPPIN with its interactor.

In the crystal structure KlAtg21 forms a seven-bladed $\beta$-propeller which is a typical fold for WD40-reapeat proteins (Xu and Min, 2011). Furthermore, the $\beta$-propeller exhibits a non-velcro closure which is rare among WD40-reapeat proteins but was already observed for the other PROPPINs Atg18 and Hsv2 (Xu and Min, 2011; Krick et al., 2012; Baskaran et al., 2012; Watanabe et al., 2012; Scacioc et al., 2017). The crystal structure further revealed that two KlAtg21 molecules are linked by the CCD of one AgAtg16 dimer which correlates with the proposed interaction mode of Atg21 and Atg16 in S. cerevisiae (Figure 2.13; Juris et al., 2015; Krick and Thumm, 2016). In the crystal structure, the CCD of AgAtg16 faces the bottom sides of the two 
$\beta$-propellers of KlAtg21, in close proximity to the blades 1, 2 and 3. This suggested that the loops and $\beta$-strands of blades 1,2 and 3 located on the bottom side of KlAtg21 potentially contribute to the interaction with AgAtg16. Direct interactions between amino acids of KlAtg21 and AgAtg16 could not be observed due to the low resolution of the data (only 4.0 Å; Metje, 2017). Therefore, a set of corresponding, conserved and mainly charged residues of Atg21 and Atg16 were chosen for a detailed analysis on their contribution to the interaction of both proteins (Table 4.1).

A variety of performed in vivo and in vitro experiments, revealed the formation of a single salt bridge between Atg21 R151 and Atg16 D101. This salt bridge is essential for targeting Atg16 to the PAS and for an efficient progression of the Cvt-pathway (chap. 4.1.1.2). Surrounding amino acids like Atg21 D28, Atg21 K130 or Atg16 K94 may stabilize the salt bridge by contributing to the net charge on the surface of both proteins but are dispensable for the interaction (chap. 4.1.1.3).

The relevance of Atg21 R151 and Atg16 D101 is further supported by gel filtration experiments with recombinant purified complexes of KlAtg21 and the CCD of AgAtg16 which were performed by Dr. Janina Metje. Upon mutation of either of the corresponding residues KlAtg21 R103 or AgAtg16 D78, the interaction of both proteins is lost. This indicates that the interaction mechanism of Atg21 and Atg16 is conserved among their yeast orthologues and that the CCD of Atg16 is sufficient for the interaction (Metje, 2017). Furthermore, the formation of the salt bridge seems to be sufficient to facilitate the interaction between Atg21 and Atg16 orthologues from different species.

In sequence alignments Atg21 R151 aligns with R108 of hWIPI2b (Figure 4.2; Wilson et al., 2014). Both arginines are located on loop $3 \mathrm{AB}$ of the respective $\beta$-propeller (Figure 4.3; Dooley et al., 2014). Furthermore, hWIPI2b R108 is also required for the interaction with hAtg16L1. It directly binds to hAtg16L1 E230 probably by forming a salt bridge suggesting that not only the function of PROPPINs in Atg16 interaction but also the interaction mechanism itself is conserved from yeast to human (Dooley et al., 2014). Interestingly, mammalian Atg16 orthologues harbor an additional large WD40-repeat domain at their C-terminus (Wilson et al., 2014). In contrast to ScAtg16, E230 of hAtg16L1 is located in the WD40-repeat 
domain and not the CCD of hAtg16L1. Therefore, in contrast to Atg21, WIPI2b does not directly interact with the CCD of hAtg16L1 although it was shown that the CCD is required for the interaction (Dooley et al., 2014). The acquired WD40-repeat domain of hAtg16L1 seems to have adapted the binding mechanism of the CCD of yeast Atg16. In line, the dimerizing CCD of Atg16 orthologues is functionally conserved, however sequentially they show a high divergence (Wilson et al., 2014). Interestingly, although interacting with hWIPI2b, the WD40-repeat domain of hAtg16L1 is not essential for starvation-induced autophagy but is involved in the clearance of Salmonella (Fujita et al., 2009; Fujita et al., 2013).

Apart from Atg16 D101 also the neighboring Atg16 E102 is required for the interaction with Atg21 (Juris et al., 2015). Likewise, the hAtg16L1 E226 in proximity to E230 also participates in the interaction with hWIPI2b (Dooley et al., 2014). So far, for both Atg16 E102 and hAtg16L1 E226 no directly interacting amino acid could be identified on the surface of Atg21 or hWIPI2b respectively (chap. 4.1.1.2; Dooley et al., 2014). In the crystal structure of the KlAtg21-AgAtg16 complex, the built-in alanine side chain of the corresponding E102 residue of AgAtg16 and the corresponding R151 side chain of KlAtg21 have a distance of $9.5 \AA ̊$. . Considering that both, glutamatic acid and arginine, have significantly longer side chains than the alanines in the crystal structure, Atg16 E102 most likely is much closer to Atg21 R151. It could provide an additional negative charge on the surface of Atg16 that supports the electrostatic interaction of both proteins. A direct interaction between Atg21 R151 and Atg16 E102 could not be confirmed in this study (chap. 4.1.1.2). The formation of a second salt bridge between Atg16 E102 and Atg21 K152 is also very unlikely since the respective alanine side chains in the crystal structure of the KlAtg21-AgAtg16 complex have a distance of $12.1 \AA$ A. Furthermore, the performed charge-change experiments revealed that the combination of the mutants Atg16 E102R and Atg21 K152E neither reconstituted the interaction of both proteins nor restored the function of the Cvt-pathway (chap. 4.1.1.2).

Mutational analyses in combination with different in vivo and in vitro assays revealed that mainly blades 2 and 3 of the PROPPIN $\beta$-propellers are involved in the binding of interactors (Table 5.1). Facing away from the membrane binding FRRG- 
motif, blades 2 and 3 are possibly best accessible for interactions (Figure 2.12). This seems to be a conserved feature of PROPPINs from yeast to mammals.

Table 5.1: List of the reported interacting regions between PROPPIN-family members and their interactors

\begin{tabular}{llll}
\hline PROPPIN & Interactor & Interacting loop & References \\
\cline { 2 - 4 }$A \operatorname{Atg} 21$ & Atg8 & 2D to 3A & (Juris et al., 2015) \\
\cline { 2 - 4 } & Atg16 & 3AB & This study \\
\multirow{2}{*}{ Atg18 } & Atg2 & 2AB, 2BC and 2D to 3A & $\begin{array}{l}\text { (Watanabe } \text { et al., 2012; Rieter } \text { et } \\
\text { al., 2013) }\end{array}$ \\
\hline hWIPI2b & hAtg16L1 & 3AB & (Dooley et al., 2014) \\
\hline hWIPI2 & hRAB11A & 3BC and $\beta$-strand 3C & (Puri et al., 2018) \\
\hline hWIPI4 & hAtg2B & 3D to 4A & (Zheng et al., 2017) \\
\hline
\end{tabular}

Interestingly, Atg21 and Atg18 use the same loop (2D to 3A) to interact with Atg8 and Atg2 respectively (Rieter et al., 2013; Juris et al., 2015). Furthermore, Atg21 R151 which is responsible for the interaction with Atg16 is conserved among all yeast PROPPINs. However, so far neither Atg18 nor Hsv2 have been reported to interact with Atg16 suggesting that there must be additional factors that target an interactor specifically to one PROPPIN. For example, Atg21 K152 is only conserved among Atg21 orthologous and also influenced the interaction of Atg21 and Atg16 in GFP-TRAP experiments (chap. 4.1.1.2). Surrounding amino acids like Atg21 K152 could therefore contribute to the specific recognition of an interactor.

Based on the crystal structure of the KlAtg21-AgAtg16 complex, Dr. Karin Kühnel and Dr. Janina Metje were able to generate a model of the overall organization of the Atg8 lipidation complex in yeast (Figure 5.1 A; Metje, 2017). The known structures of Atg8 and the Atg12 Atg5 conjugate were included into the model by superimposition. The Atg8 lipidation complex shows a structural relation to the LC3 lipidation complex in mammals (Figure 5.1 B; Wilson et al., 2014). This structural conservation of the lipidation complexes further confirms their importance during autophagy.

Furthermore, the suggested structure of the Atg8 lipidation complex perfectly correlates with the proposed model by Juris et al. (Figure 2.13; Juris et al., 2015; Krick and Thumm, 2016). In this model Atg21 has no enzymatic activity but acts as a scaffold on the autophagic membrane which is in line with the typical function of WD40-repeat proteins. So far, no member of the WD40-repeat family shows an intrinsic enzymatic activity (Jain and Pandey, 2018). 
Strikingly, due to the orientation of the FRRG-motif of Atg21 in the modeled structure of the Atg8 lipidation complex, the association with the concave side of a bended membrane is implicated. An observation which will be discussed in the following chapter.
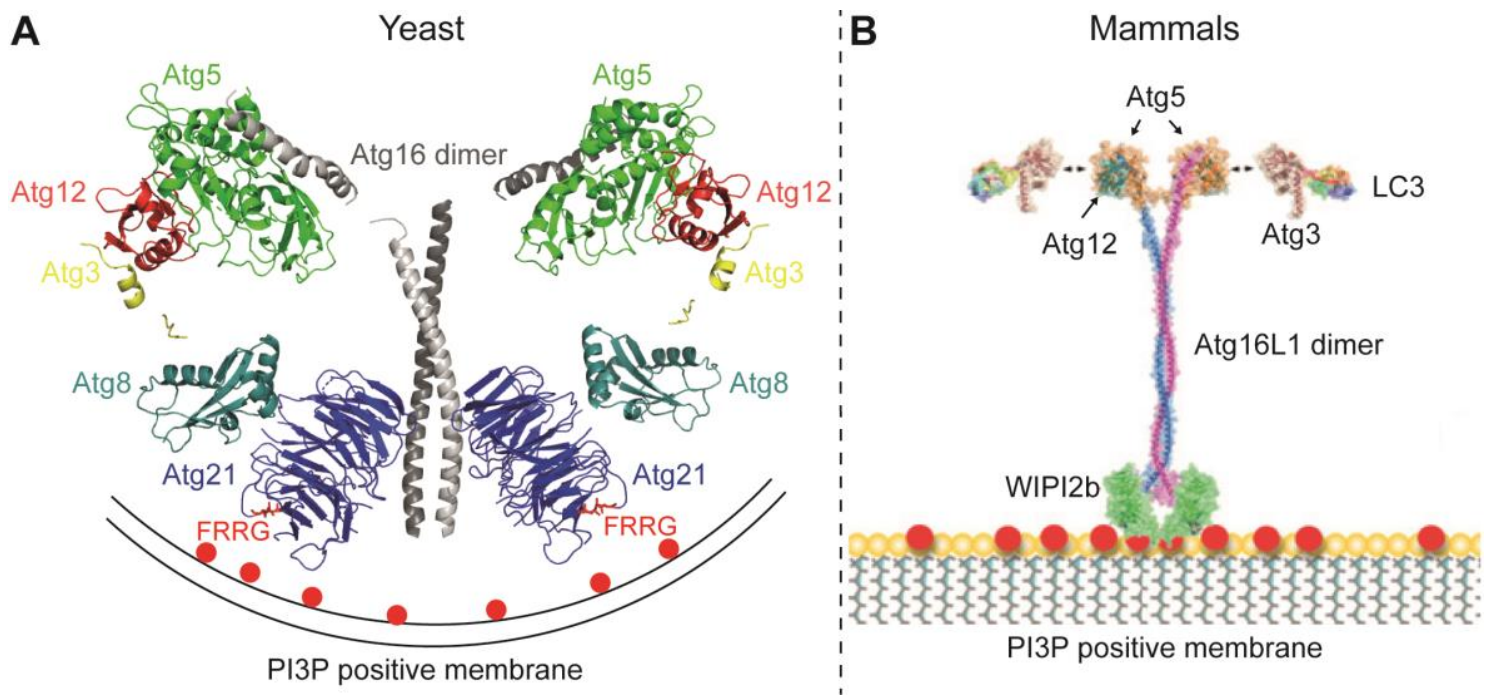

Figure 5.1: Models of the structure of the Atg8 lipidation complex in yeast and mammals

(A) Proposed model of the spatial arrangement of the Atg8 lipidation complex in yeast generated by superimposition of the available crystal structures of Atg8 (turquoise), Atg3 (yellow), Atg12 (red) Atg5 (green), Atg16 (grey) and Atg21 (blue). Structures are represented as cartoons. The conserved FRRG-motif of Atg21 is shown as sticks and colored in red. The potential binding of the complex to the concave side of a bended PI3P (red) positive membrane is implicated. (modified from Metje (2017)) (B) A model of the mammalian Atg3-LC3-Atg12-Atg5-Atg16L1-WIPI2b complex that mediates LC3 lipidation at PI3P (red) positive membranes. The model is based on the following crystal structures: KmHsv2 for WIPI2b (green; PDB code: 3VU4), ScAtg16 (PDB code: 3A7P), human Atg3-Atg12-Ag5-Atg16 (PDB code: 4NAW), ScAtg7-ScAtg3-ScAtg8 (PDB code: 4GSL), human LC3B (PDB code: 2ZJD). (modified from Wilson et al. (2014))

\subsubsection{Models of the membrane association of the Atg21-Atg16 complex}

In the crystal structure the two KlAtg21 molecules tilt with the upper part of their bottom side towards the CCD of AgAtg16, thereby adopting a reverse V shape (Figure $4.1 \mathrm{~A}$ ). This conformation probably enables the direct interaction of Atg21 R151 and Atg16 D101. In this conformation, the conserved membrane binding FRRG-motif of KlAtg21 which is located on $\beta$-strand 5D and the connecting loop to $\beta$-strand 6A faces away from AgAtg16 (Figure 4.3; Metje, 2017). This orientation of the PI3P binding pockets implicates that the Atg21-Atg16 complex preferably binds to membranes with a concave shape or that the complex is even able to induce membrane curvature. 
The PI3P positive inner membrane of the cup-like phagophore displays such a concave shape suggesting it as a target membrane of the Atg21-Atg16 complex. However, in mammalian cells the Atg12 Atg5/Atg16 complex resides exclusively on the convex site of the cup-like phagophore where it is thought to form a coat-like membrane scaffold together with Atg8-PE (Mizushima et al., 2001; Mizushima et al., 2003; Kaufmann et al., 2014). Furthermore, neither the Atg12 Atg5/Atg16 complex nor Atg21 are transported to the vacuole via autophagosomes and are therefore likely released shortly before or after autophagosome completion (Mizushima et al., 2001; Mizushima et al., 2003; Juris, 2014). Accordingly, the association of the Atg21Atg16 complex with the inner membrane of the phagophore is very unlikely.

Therefore, two models can be proposed for the membrane binding of the Atg21-Atg16 complex (Figure 5.2). In the first model, the Atg21-Atg16 complex resides on the rim of the growing phagophore, probably slightly tilted towards the inner face of the phagophore by sensing the concave-shape of the inner membrane (Figure $5.2 \mathrm{~A}$ ). This would target the lipidation of Atg8 to the rim of the forming autophagosome. After conjugation, Atg8-PE could be easily distributed to the inner and outer membrane of the phagophore to fulfill its function in cargo recognition (inner membrane; Farré and Subramani, 2016) and formation of a coat-like scaffold structure together with the Atg12 Atg5/Atg16 complex (outer membrane; Kaufmann et al., 2014). During the expansion of the phagophore, the single salt bridge between the Atg21 and Atg16 molecules could provide some flexibility to adapt the angle of the two Atg21 molecules to the varying membrane curvature.

Interestingly, in vitro studies revealed that recombinantly purified Atg18 is able to induce tubulation and scission of GUVs. The observed membrane bending and scission is dependent on the formation of an amphipathic $\alpha$-helix in loop 6CD of the Atg18 $\beta$-propeller (Gopaldass et al., 2017). The hydrophobic loop 6CD was already reported to support the membrane association of PROPPINs as an additional membrane anchor (Busse et al., 2015). Although the 6CD loop shows a high sequential variation among PROPPIN members of all species, the ability to form an amphipathic $\alpha$-helix seems to be well conserved (Gopaldass et al., 2017). Amphipathic $\alpha$-helices are protein domains which can partially penetrate into membranes and thereby change the membrane structure. They facilitate a 
peripheral membrane association of the protein. An asymmetrical distribution of such membrane perturbations can induce a local membrane curvature (Kozlov et al., 2014). Atg18 was observed to oligomerize at the membrane which in combination with insertion of the amphipathic $\alpha$-helix would facilitate membrane bending (Scacioc et al., 2017; Gopaldass et al., 2017). These findings support the hypothesis that the Atg21-Atg16 complex might induce membrane curvature at the rim of the growing phagophore.

Remarkably, in vitro experiments with recombinant purified KlAtg21 revealed that KlAtg21 highly induces the tubulation of large unilamellar vesicles (unpublished data). This indicates that Atg21 is indeed able to bend membranes. The experiments were performed in parallel to this study by the cooperation partner Dr. Benjamin Kroppen in the research group of Prof. Dr. Michael Meinecke (Department of Cellular Biochemistry, University Medical Center Göttingen).

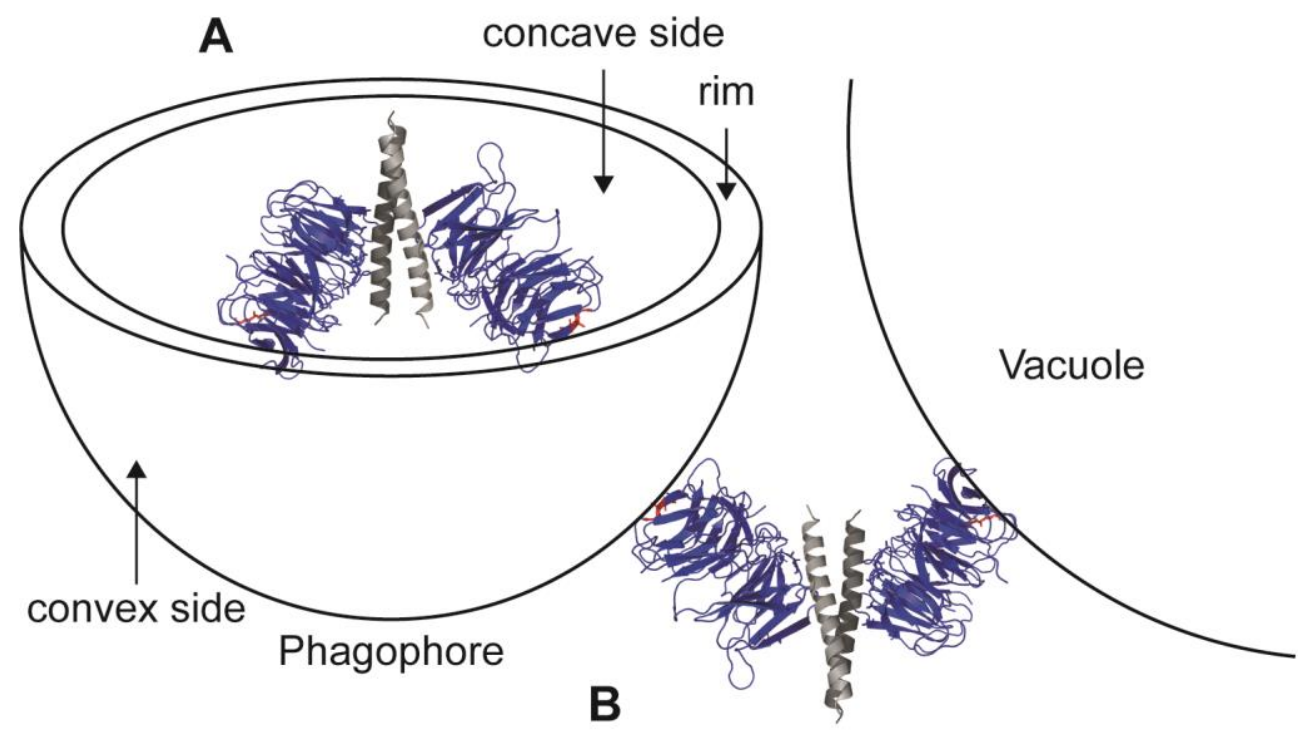

Figure 5.2: Two proposed models of the membrane binding of the Atg21-Atg16 complex

Cartoon representation of KlAtg21 (blue) in complex with the CCD of AgAtg16 (grey). The membrane binding FRRG-motif of KlAtg21 is colored in red. From the orientation of the FRRG-motif in the crystal structure two models for the membrane binding of the KlAtg21-AgAtg16 complex are proposed: (A) The Atg21-Atg16 complex resides on the rim of the growing phagophore, probably slightly tilted towards the inner face of the phagophore by sensing the concave-shape of the inner membrane. This subsequently targets the assembly of the Atg8 lipidation complex and further the conjugation of Atg8-PE to the rim of the forming autophagosome. Atg8-PE can then be distributed to the inner membrane for cargo recruitment or to the outer membrane for the formation of a coat-like scaffold structure together with the Atg12 Atg5/Atg16 complex. (B) The Atg21-Atg16 complex binds to two opposing PI3P- or PI3,5 $\mathrm{P}_{2}$-positive membranes. Thereby, the growing phagophore could be tethered to the PI3P- and PI3,5 $\mathrm{P}_{2}$-enriched vacuolar membrane. Membrane binding is probably supported by the membrane tethering ability of Atg5 which binds to Atg16. The Atg8 lipidation complex is assembled in between the two membranes. On the side of the autophagic membrane Atg8 is conjugated to $\mathrm{PE}$ and then distributed to the inner and outer membrane by diffusion. On the vacuolar 
side of the Atg8 lipidation complex additional mechanisms would probably prevent the generation of non-functional Atg8-PE.

In the second proposed model the Atg21-Atg16 complex binds to two opposing PI3P- or PI3,5P2-positive membranes which would lead to the tethering of both membranes (Figure 5.2 B). Apart from autophagic membranes, PI3P and PI3,5P 2 are enriched on endosomal and vacuolar membranes (De Craene et al., 2017). Since the PAS is frequently located close to the vacuole, the vacuolar membrane could be a potential target for membrane tethering by the Atg21-Atg16 complex. The binding of the opposing autophagic and vacuolar membrane could be supported by Atg5 which has a membrane tethering ability as well as an affinity for negatively charged membranes (Romanov et al., 2012). The membrane binding of Atg5 is required for autophagy progression after its recruitment to the PAS but precedes the Atg8 lipidation (Romanov et al., 2012). This could support a model where the Atg21Atg12 Atg5/Atg16 complex would first tether the autophagic membrane to the vacuolar membrane and subsequently assembles the Atg8 lipidation complex in between the two opposing membranes by recruiting the Atg8 Atg3 conjugate.

Remarkably, the Atg18-Atg2 complex was recently reported to establish a functional contact site between the phagophore and the ER (Gomez-Sanchez et al., 2018; Kotani et al., 2018). Thereby, Atg18 is thought to be responsible for the PI3Pdependent targeting of the complex to the phagophore while Atg2 mediates the tethering of the autophagic membrane to the ER. Atg2 comprises two membrane binding domains of which the C-terminal domain forms an amphipathic $\alpha$-helix that in addition to Atg18 is required for association with the phagophore. The N-terminal membrane binding domain is thought to bind to the ER membrane (Kotani et al., 2018). Interestingly, the human orthologous hWIPI4 and hAtg2B form a club-shaped structure reminiscent of the HOPS tethering complex. The hWIPI4hAtg2B complex is therefore assumed to have a tethering function which is in line with the function of the Atg18-Atg2 complex (Zheng et al., 2017; Kotani et al., 2018). This indicates that PROPPINs indeed participate in membrane tethering and supports the second model for membrane association of the Atg21-Atg16 complex. According to the second model, the Atg8 lipidation complex would be able to generate Atg8-PE on the autophagic as well as the vacuolar membrane. Since Atg8 exclusively localizes to the phagophore, additional mechanisms would be required 
to either prevent the generation of Atg8-PE at the vacuolar membrane or to remove non-functional Atg8-PE from the vacuole. Atg8 lipidation could be prevented by an additional interaction partner of Atg21 at the vacuolar membrane. Upon binding the interactor could mask the loop 2D to 3A of Atg21 and thereby inhibit the recruitment of Atg8. In addition, the interaction of Atg21 with a vacuolar protein could further stabilize the tethering of the two membranes. In this study, Vac8 was co-isolated with GFP-Atg21 in GFP-TRAP experiments indicating that Atg21 might directly interact with vacuolar membrane proteins. Non-functional Atg8-PE at the vacuolar membrane could be removed by Atg 4 which is able to hydrolyze the bond between Atg8 and PE and is reported to remove randomly generated Atg8-PE from non-autophagic membranes (Kirisako et al., 2000; Nakatogawa et al., 2012a).

All together the second model for membrane association of the Atg21-Atg16 complex is highly speculative and only supported by the membrane affinity of Atg5 and the tethering function of the Atg18-Atg2 complex (Romanov et al., 2012; GomezSanchez et al., 2018; Kotani et al., 2018). Nevertheless, both models do not exclude each other. The Atg21-Atg12 Atg5/Atg16 complex could exhibit two functions, namely the Atg8 lipidation at the rim of the phagophore and the tethering of autophagic and vacuolar membrane.

\subsubsection{Dynamics of the Atg21-Atg16 complex formation}

Intermolecular interactions including salt bridges or hydrophobic interactions contribute to the stability of protein complexes and thereby also contribute to the activity of the protein complex. The activity of protein complexes is further regulated by their dynamical association and dissociation. Mechanisms that induce the formation or disassembly of protein complexes can define the spatial and temporal activity of the complex. For example, the Atg8 lipidation complex should be specifically assembled at the autophagic membrane to prevent the coupling of Atg8 to PE at non-autophagic membranes. After the lipidation, Atg8-PE needs to be released from its interaction with Atg21, to enable it to fulfill its functions in cargo recruitment and scaffold formation (Kaufmann et al., 2014; Farré and Subramani, 2016). So far, the dynamics of association and dissociation of the Atg8 lipidation complex are still elusive. 
Two models can be assumed for the association of the Atg8 lipidation complex. Either all components assemble in the cytosol and are targeted to the autophagic membrane in one complex. Or Atg21 first binds PI3P-dependent to the PAS and afterwards recruits the $\operatorname{Atg} 12 \sim \operatorname{Atg} 5 / \operatorname{Atg} 16$ complex as well as the Atg8 Atg3 conjugate. In this study four different approaches were used to elucidate the sequence of events that lead to the formation of the Atg8 lipidation complex and its membrane association. To be able to distinguish between the membrane bound complex and the potentially formed cytosolic complex the lipid-binding deficient Atg21 FTTG mutant was used.

Unfortunately, the combination of the four used approaches did not result into a clear model for the association of the Atg8 lipidation complex (chap. 4.1.2). The FCCS measurements revealed that there is no complex formation between Atg21 FTTG and Atg16 in the cytosol suggesting that Atg21 needs to be recruited to the membrane before its interaction with Atg16 (chap. 4.1.2.2). This would favor the model that the Atg8 lipidation complex is assembled at the PI3P-positive membrane and not in the cytosol. The performed GFP-TRAP experiments partly support this model. The interaction between Atg21 FTTG and Atg16 was significantly reduced to about $75 \%$ of the WT proteins (chap. 4.1.2.1) suggesting that membrane binding supports the interaction of both proteins. Here, it must be considered that compared to the input, only a small fraction of Atg16 (probably only some percent) is coisolated with WT Atg21. The observed interaction of Atg21 and Atg16 in the GFPTRAP approach could therefore reside within the detection limit of the FCCS measurements. It is possible that there might be 1 to $2 \%$ bound protein complexes which are not visible with the FCCS measurements (personal communication of Dr. Narain Karedla). Therefore, the GFP-TRAP experiments might even highly support the FCCS measurements.

This was however opposed by the results of the split-ubiquitin assay. Here, Atg21 FTTG showed a stronger interaction with Atg16 compared to WT Atg21 suggesting that the complex of Atg21 and Atg16 is stabilized in the cytosol (chap. 4.1.2.3). To complicate the situation even more, the results of the BioID assay favored both models depending on the used bait (chap. 4.1.2.4). 
Among the used approaches, FCCS is the most suitable method for the evaluation of protein dynamics as it directly measures the fluctuation of fluorescently-labeled proteins in the observation volume. Thereby, the proteins are monitored in their native environment (Bacia et al., 2006). The split-ubiquitin assay is another approach to determine protein interactions in vivo (Johnsson and Varshavsky, 1994). However, the reassembly of the two halves of ubiquitin may arrest the analyzed complex which could lead to an overestimation of the interaction. This could further result into the detection of false-positive interactions and would explain the strong interaction between Atg21 FTTG and Atg16 in the split-ubiquitin assay. Due to its release from the membrane, Nub-Atg21 FTTG might be better accessible by Atg16-Cub for the reconstitution of ubiquitin. Therefore, the FCCS measurements seem to be more reliable compared to the split-ubiquitin assay.

The BioID assay is another approach for the detection of protein interactions or proximities in vivo. In contrast to the split-ubiquitin assay it does not rely on the reconstitution of two halves of a protein but covalently labels interacting proteins with biotin (Roux et al., 2012). Therefore, the BioID assay seems to be better suited for the detection of dynamic interactions compared to the split-ubiquitin assay. However, the results of the BioID assay were the most contradictory ones. Using the Atg21 FTTG mutant as the bait, the biotinylation of Atg16 is highly reduced compared to WT Atg21 suggesting that there is no interaction of both proteins in the cytosol. This supports the results of the FCCS measurements. In contrast, the biotinylation of Atg21 is highly increased after the mutation of the FRRG-motif when Atg16 is used as the bait (chap. 4.1.2.4). This indicates that the interaction of both proteins is even stronger in the cytosol and supports the results of the splitubiquitin assay. Strikingly, the biotinylation of WT Atg21 is very low in presence of BirA*-Atg16 and Atg16-BirA* suggesting that BirA* might be less active in the membrane bound complex. However, Atg5 and the Atg12 Atg5 conjugate were efficiently biotinylated by both BirA* fusions of Atg16. Therefore, the reason for discrepancy remains unsolved.

The FCCS measurements seem to provide the most reliable results and are supported by the GFP-TRAP experiments, suggesting that the Atg8 lipidation complex might be preferably assembled at the autophagic membrane. However, due 
to the discrepancies between the results of the performed experiments the exact mechanism of complex assembly of the Atg8 lipidation complex will need further investigation.

Interestingly, a similar discrepancy was observed for the formation of the Atg18-Atg2 complex. Obara et al. (2008b) reported that the deletion of ATG18 or the mutation of its FRRG-motif leads to the release of Atg2 from the PAS. In contrast, Rieter et al. (2012) showed that Atg2 is recruited to the PAS independent of Atg18. Two recent studies revealed that Atg2 is indeed able to bind membranes independent of Atg18 and harbors two membrane binding domains (GomezSanchez et al., 2018; Kotani et al., 2018). However, Atg18 seems to be required for the proper PI3P-dependent localization of Atg2 to the autophagic membrane (Kotani et al., 2018).

\subsection{Determination of the precise localization of proteins at the growing phagophore}

In yeast cells using fluorescence microscopy, Atg proteins are observed to localize at least transiently to a distinct perivacuolar dot defined as the PAS. Microscopic analyses revealed that the Atg proteins are recruited in a hierarchical manner (Suzuki et al., 2001; Suzuki et al., 2004). However, due to the small size of the forming phagophore and the resulting autophagosome (400-900 $\mathrm{nm}$ in diameter), the exact temporal and spatial localization of Atg proteins at the growing phagophore is difficult to determine (Takeshige et al., 1992; Baba et al., 1994).

The resolution of widefield and confocal laser scanning microscopes is restricted by the diffraction limit of light. Under optimal conditions the best theoretical resolution for visible light is $190 \mathrm{~nm}$ but it is significantly lower under non-perfect conditions (200-250 nm or lower) (Turkowyd et al., 2016). Due to these resolution limits, it is nearly impossible to distinguish between the PAS, the elongated phagophore or the already closed autophagosome using normal fluorescence microscopy. A variety of methods have evolved to overcome resolution limits of the normal fluorescence microscopy, including transmission electron microscopy.

Electron microscopy can resolve structures within a nanometer scale and was initially used to identify and characterize autophagosomes and Cvt-vesicles 
(Takeshige et al., 1992; Baba et al., 1994; Baba et al., 1995; Baba et al., 1997). In combination with immuno-gold labeling, electron microscopy was further used to determine the localization of Atg proteins at the phagophore and autophagosomes (Kirisako et al., 1999; Mizushima et al., 2001; Mizushima et al., 2003). However, electron microscopy is not applicable for live cells and therefore not suitable to analyze dynamic processes in the cell like the formation of the phagophore.

In parallel, three independent groups reported about approaches which enabled them to determine the precise localization of Atg proteins at the growing phagophore using normal fluorescence microscopy (Suzuki et al., 2013; Sun et al., 2013; Graef et al., 2013). One of these approaches, the APE1-overexpression assay, was used in this study to analyze the precise localization of Atg21 and other proteins at the growing phagophore. All three approaches will be discussed in the following chapter.

\subsubsection{Advantages and disadvantages of the APE1-overexpression assay and comparable approaches}

Using the APE1-overexpression assay, the high expression levels of APE1 lead to the formation of highly enlarged Ape1-complexes referred to as GACs, which exceed the capacity of autophagosomes and are therefore not transported to the vacuole. Nevertheless, the autophagic machinery is recruited to the GACs forming a phagophore in the attempt to enclose the giant cargo. Phagophore formation can be visualized as a cup-like structure that engulfs the GAC using GFP-Atg8 as a marker. Due to their larger mass GACs are less mobile in the cell. Therefore, the dynamical expansion of the phagophore can be easily followed over time. The cup-shaped phagophore expands until it reaches its maximum size. Here it remains for a longer period before it is disassembled (Suzuki et al., 2013). Atg proteins display three distinct localization patterns at the growing phagophore. They either localize to a single dot-like structure proximal to the vacuole (Atg13, Atg17 and the PI3-kinase complex), reside at the edges of the phagophore (Atg9 and the Atg18-Atg2 complex) or cover the whole phagophore (Atg1, Atg8 and the Atg12 Atg5/Atg16 complex) (Suzuki et al., 2013). 
The expression level of APE1 seems to be essential for the formation of GACs. When only moderately overexpressed pApe1 accumulates under nutrient rich conditions but it is efficiently transported to the vacuole upon starvation (Geng et al., 2008; Suzuki et al., 2013). A reason for this is probably that the larger autophagosomes (400-900 $\mathrm{nm}$ in diameter) have a higher cargo capacity compared to the smaller Cvt-vesicles (140-160 nm in diameter) (Takeshige et al., 1992; Baba et al., 1994; Baba et al., 1997). The formation of GACs is achieved by the expression of high-copy plasmid-encoded APE1 under the control of the CUP1 promoter (Suzuki et al., 2013). Cup1 is a metallothionein which facilitates the resistance to high copper concentrations by binding copper ions. CUP1 transcription is activated in response to high concentrations of copper ions (Rutherford and Bird, 2004). The expression of genes under control of the CUP1 promoter can therefore be induced by the addition of $\mathrm{CuSO}_{4}$ to the medium. However, high concentrations of copper can be toxic for the cell. This was noticeable by a drastic reduction in cell growth when using the recommended concentration of $250 \mu \mathrm{M} \mathrm{CuSO} 4$ to induce the APE1 expression (chap. 4.2.1; Suzuki et al., 2013). In this study, the concentration of $\mathrm{CuSO}_{4}$ was therefore reduced to $100 \mu \mathrm{M}$ which only slightly affected cell growth. Furthermore, $100 \mu \mathrm{M} \mathrm{CuSO}_{4}$ were sufficient to induce the formation of GACs and GFP-Atg8 positive phagophores which were visualized by fluorescence microscopy (chap. 4.2.1).

GACs are artificial cargos for the autophagic machinery which would not exist in the cell under normal conditions. Furthermore, pApe1 is a selective cargo of the Cvt-pathway that is recognized and targeted by its specific receptor Atg19 for the transport to the vacuole (Leber et al., 2001; Scott et al., 2001). Apart from Atg19, Atg11 is required for a proper localization of the Cvt-complex to the PAS (Kim et al., 2001; Shintani et al., 2002). Atg19 and Atg11 are both dispensable for unselective autophagy indicating that there are differences in the mechanism of phagophore assembly between selective and unselective types of autophagy (Leber et al., 2001; Scott et al., 2001; Kim et al., 2001; Shintani et al., 2002). This must be considered when evaluating the precise localization of Atg proteins at the phagophore using the $A P E 1$-overexpression assay. Furthermore, the assay is only suitable to analyze early stages of autophagy since a complete autophagosome cannot be formed around the GACs. 
In contrast to the APE1-overexpression assay, Graef et al. (2013) were able to visualize GFP-Atg8 positive cup-like and ring-like structures, which represent the phagophore and the closed autophagosome respectively, simply by inducing autophagy with rapamycin $(400 \mathrm{ng} / \mathrm{ml})$. Their approach did not require any overexpression of APE1. To focus on unselective autophagy, they even used an ATG19 deletion strain that is not able to transport pApe1 to the vacuole (Leber et al., 2001; Scott et al., 2001; Graef et al., 2013). Similar to Suzuki et al. (2013) they colocalized Atg proteins with the growing phagophore and observed distinct localization patterns. However, there are some discrepancies to the observations of Suzuki et al. (2013). Graef et al. (2013) reported that Atg14 and Atg13 localize to a single dot at the periphery of the phagophore while Atg2 resides at its edge which is comparable with the findings of Suzuki et al. (2013). Atg1, Atg5 and Atg16 however did not cover the phagophore as it was reported by Suzuki et al. (2013) but localized to a dot-like structure at the periphery of the phagophore similar to Atg14 and Atg13 (Suzuki et al., 2013; Graef et al., 2013).

Interestingly, Atg19 was recently reported to be able to directly interact with Atg5 via a mechanism which is reminiscent to the AIM-dependent interaction of Atg19 with Atg8. This interaction seems to be important for the recruitment of the Atg12 Atg5/Atg16 complex to the PAS and facilitates the lipidation of Atg8 during selective autophagy (Fracchiolla et al., 2016). This could explain a different distribution of the Atg12 Atg5/Atg16 complex in an atg194 strain compared to a WT strain.

In this study, some small ring-like structures were observed after the induction of autophagy with rapamycin $(400 \mathrm{ng} / \mathrm{ml})$ in a WT strain expressing plasmid-encoded GFP-ATG8 under the control of its endogenous promoter (data not shown). Nevertheless, these structures did not reach the size of those reported by Graef et al. (2013). The observed structures were too small to determine the precise localization of other proteins at the phagophore/autophagosome. Only with a highly increased amount of rapamycin $(1 \mu \mathrm{g} / \mathrm{ml})$ some bigger ring-like structures were observed (data not shown). The reason for the different observations compared to Graef et al. (2013) remains unclear but probably correlates with the deletion of 
ATG19. Due to the unsatisfying results this approach was discarded and this study focused on the APE1-overexpression assay.

In a genetic screen for the identification of Atg proteins in the fission yeast S. pombe, Sun et al. (2013) discovered an autophagy defective mutant (ctl14) which produced enlarged cup-shaped CFP-Atg8-positive structures after prolonged starvation ( $>4 \mathrm{~h}$ ). When colocalizing Atg proteins to the CFP-Atg8-positive structures they displayed distinct localization patterns similar to those observed with the APE1-overexpression assay (Suzuki et al., 2013; Sun et al., 2013). CTL1 encodes a member of the choline transporter-like (CTL) protein family and is a predicted transmembrane protein. It partially localizes to the PAS and seems to interact with Atg9. The role of Ctl1 in autophagy and the reason for the formation of the enlarged CFP-Atg8-positive structures in its absence remain elusive. The S. cerevisiae homolog Pns1 is a protein of unknown function (Sun et al., 2013). It would be interesting to investigate whether Pns1 is also required for autophagy and whether its deletion also results in the formation of enlarged Atg8-positive structures. This could provide another tool for the detailed investigation of the spatial organization of the phagophore that is independent of a selective cargo.

\subsubsection{Under starvation conditions Atg21 restricts Atg8 lipidation to the contact site of vacuole and phagophore}

Atg21 and WIPI2b define the site of Atg8 and LC3 lipidation respectively. Thereby, the PI3P-binding of both $\beta$-propellers is essential to target the lipidation complexes to the autophagic membrane (Dooley et al., 2014; Juris et al., 2015). In this study, the APE1-overexpression assay was used to determine the precise localization of Atg21 at the phagophore to further characterize the lipidation site of Atg8. Under starvation conditions Atg21-YFP localized to a single dot in proximity to the vacuolar membrane suggesting that Atg8 lipidation is restricted to the contact site between vacuole and phagophore (chap. 4.2.2). Remarkably, none of the other components of the Atg8 lipidation complex shares this localization with Atg21. In this study, Atg8, Atg16 and Atg3 were instead observed to cover the whole phagophore, which perfectly correlates with their reported localization (chap. 4.2.1 and 4.2.3; Suzuki et al., 2013; Ngu et al., 2015; Sakoh-Nakatogawa et al., 2015). 
If Atg21 really defines the site of Atg8 lipidation the other components of the lipidation complex will have an additional Atg21-independent function that retains them at the phagophore after the conjugation reaction. Atg8-PE and the Atg12 Atg5/Atg16 complex form a coat-like membrane scaffold on the outer side of the phagophore which could explain their Atg21-independent distribution on the phagophore (Kaufmann et al., 2014). Atg3 was not observed as part of this scaffold structure. Instead, it seems to compete with the Atg12 Atg5 complex for the interaction with Atg8 (Kaufmann et al., 2014).

Interestingly, Atg3 contains an AIM which is important for its interaction with Atg8 and is required for an efficient in vitro conjugation of Atg8 to PE in the absence of the Atg12 Atg5 conjugate. However, the Atg3 AIM is dispensable for the generation of Atg8-PE in vivo. Furthermore, the AIM is required for the Cvt-pathway but is not essential for unselective autophagy (Yamaguchi et al., 2010). This corresponds to the findings of this and another study, that cells expressing the ATG3 AIM mutant are still able to assemble the phagophore (chap. 4.2.3; Sakoh-Nakatogawa et al., 2015). Atg3 is essential for the lipidation of Atg8 and therefore needs to at least transiently locate to the phagophore (Ichimura et al., 2000). However, upon mutation of the AIM the association of Atg3 with the phagophore is lost (chap. 4.2.3; Sakoh-Nakatogawa et al., 2015) suggesting that after Atg8 conjugation the Atg3 AIM mutant rapidly dissociates from the phagophore. This further indicates that the AIM-dependent interaction with Atg8 is essential for Atg3 to remain associated with the phagophore after the lipidation reaction.

This hypothesis is further supported by the observation that in presence of endogenous Atg3 that allows the formation of a phagophore the mutation of Atg3 C234A does not abolish its association with the phagophore (chap. 4.2.3; SakohNakatogawa et al., 2015). C234 is the catalytic cysteine of Atg3 which is conjugated to Atg8 during the lipidation process. The mutant Atg3 C234A is defective in forming the Atg8 Atg3 conjugate and therefore inhibits the generation of Atg8-PE (Ichimura et al., 2000). In cells expressing only ATG3 C234A the formation of the phagophore is blocked. However, in presence of endogenous Atg3, that facilitates the phagophore assembly, Atg3 C234A is recruited to the phagophore independent of its conjugation to Atg8 (chap. 4.2.3; Sakoh-Nakatogawa et al., 2015). The association 
of Atg3 C234A with the phagophore is probably mediated by its AIM-dependent interaction with Atg8. Atg3 C234A could also interact with endogenous Atg3 at the phagophore. However, this interaction would then be dependent on the AIM of Atg3, since the Atg3 AIM mutant is not recruited to the phagophore in presence of endogenous Atg3.

In contrast to this study, Sakoh-Nakatogawa et al. (2015) reported that Atg3-GFP is not evenly distributed at the phagophore but is often enriched at the vacuolephagophore contact site. These findings would support that the lipidation of Atg8 is restricted to the vacuole-phagophore contact site. Sakoh-Nakatogawa et al. (2015) introduced the GFP-tag in between Q117 and S118 of Atg3 while in this study the GFP-tag was integrated between Atg3 D265 and G266 according to Ngu et al. (2015). Furthermore, Sakoh-Nakatogawa et al. (2015) introduced ATG3-GFP into the locus of $A T G 3$ while in this study plasmid-encoded ATG3-GFP was expressed. This could explain the differences in the observed localization of Atg3.

All together these findings suggest that Atg3 probably has a second function at the phagophore that is independent of its conjugase activity and might retain it at the phagophore after the lipidation of Atg8. The localization of Atg8-PE, the Atg12 Atg5/Atg16 complex and Atg3 all over the phagophore might represent their additional function after the lipidation reaction while the reaction itself is Atg21dependent restricted to one site of the phagophore.

Interestingly, Carlsson and Simonsen (2015) postulated a similar model for the lipidation of LC3 in mammalian cells. They suggested that LC3-PE is generated at the highly curved rim of the phagophore (Carlsson and Simonsen, 2015). This model is based on the finding that human Atg3 (hAtg3) comprises a membrane-curvaturesensing domain. hAtg3 was reported to facilitate LC3 and GABARAP lipidation only on highly curved membranes which is dependent on a N-terminal amphipathic $\alpha$-helix of hAtg3 (Nath et al., 2014). It would be interesting to investigate if this amphipathic $\alpha$-helix is also conserved among yeast Atg3 orthologous. Then Atg3 and Atg21 could act in concert to target the Atg8 lipidation to the highly curved rim of the phagophore.

In contrast to Atg21, its homolog Atg18 was observed to localize to both edges of the phagophore (chap. 4.2.4; Suzuki et al., 2013). 3D-projections of the growing 
phagophore revealed that Atg18 does not localize to single dot-like structures but resides at the rim of the phagophore. The localization of Atg18 to the highly curved rim of the phagophore corresponds to its ability to tubulate GUVs in vitro (Gopaldass et al., 2017). Furthermore, it also correlates with the first proposed model for membrane binding of the Atg21-Atg16 complex (chap. 5.1.2). This suggests that Atg21 like Atg18 resides on the rim of the phagophore but is restricted to the vacuole phagophore contact site by additional interactions with vacuolar proteins. A potential candidate for this interaction could be Vac8 (chap. 4.2.5.4). However, the localization of Atg21 to the vacuole-phagophore contact site could also support the second proposed model for membrane binding. In this model, the localization of Atg21 would be restricted due to its participation in the tethering of the autophagosomal and vacuolar membrane.

Nevertheless, the localization of both PROPPINs does not correspond to the observed and reported distribution of PI3P all over the phagophore. Using the APE1-overexpression assay, the mRFP-tagged PI3P-binding 2xFYVE-domain of mammalian Hrs was observed to cover the whole phagophore (chap. 4.2.4). This corresponds to the determined distribution of PI3P at the autophagic membrane by electron microscopy. Immuno-gold labeling revealed that PI3P predominantly resides on the inner autophagic membrane and is occasionally enriched at the edges of the phagophore (Obara et al., 2008a; Cheng et al., 2014).

At the autophagic membrane PI3P is generated by PI3-kinase complex I which localizes to the vacuole-phagophore contact site (chap. 4.2.4; Suzuki et al., 2013). From there PI3P possibly diffuses to the inner and outer membrane of the phagophore. The unequal distribution of PI3P between the two membranes of the phagophore is probably established by its hydrolysis on the outer membrane. PI3P hydrolysis is mediated by the PI3P-specific phosphatase Ymr1 and is required during the maturation of completed autophagosomes to detach Atg proteins from the outer autophagosomal membrane (Parrish et al., 2004; Cebollero et al., 2012; Cheng et al., 2014). After closure of the autophagosome the inner fraction of PI3P is transported together with the autophagosome to the vacuole (Obara et al., 2008a). According to the observations of Obara et al. (2008a), the PI3P gradient from inner to outer membrane of the phagophore seems to be already established and 
maintained during phagophore formation. This could explain that neither Atg18 nor Atg21 localize to the outer face of the phagophore. However, they do also not associate with the inner PI3P rich membrane suggesting that there must be additional mechanisms apart from PI3P binding which determine the precise localization of the PROPPINs at the phagophore.

One factor that determines the localization of PROPPINs could be the sensing of membrane curvature. Atg18 as well as Atg21 are able to form an amphipathic $\alpha$-helix in their 6CD loop which can induce and sense membrane curvature. Indeed, both proteins are able to induce membrane bending in vitro (Gopaldass et al., 2017, unpublished data). Furthermore, the localization of PROPPINs can be dependent on their interaction partners. Interestingly, Atg2 as well as the human orthologue of Atg3, which are both components of PROPPIN complexes, also comprise amphipathic $\alpha$-helices (Nath et al., 2014; Kotani et al., 2018). This indicates that sensing of membrane curvature might be a general feature of PROPPIN complexes and might be the mechanism that targets them to the rim of the phagophore.

Although Atg21 and Atg18 both localize to the rim of the phagophore, Atg18 seems to be unequally distributed (Figure 4.18) and Atg21 is restricted to the vacuolephagophore contact site. It would be interesting to determine if Atg21 excludes Atg18 from the contact site of vacuole and phagophore or if Atg21 localizes to the exclusions of Atg18 at the rim of the phagophore. This could suggest that both proteins compete for the association with the rim of the phagophore.

\subsubsection{Atg21 shows an aberrant localization in stationary cells}

Since the function of Atg8 lipidation is conserved from yeast to human and the generation of Atg8-PE is important for phagophore expansion it is astonishing that Atg21 is only essential for selective types of autophagy (Barth et al., 2002; Stromhaug et al., 2004; Meiling-Wesse et al., 2004; Xie et al., 2008; Kanki et al., 2009a; Dooley et al., 2014; Juris et al., 2015). This could indicate that during autophagy either the function of Atg21 as a scaffold in Atg8 lipidation can be taken over by another protein or the upregulation of $A T G 8$ expression after autophagy induction provides an excess of Atg8 which is sufficient to facilitate an efficient 
lipidation (Xie et al., 2008). However, the localization pattern of Atg21 at the phagophore in stationary cells raised another possible explanation.

Remarkably, Atg21 was the only analyzed protein that displayed differences in its localization between stationary and starved cells. Apart from its restriction to the phagophore-vacuole contact site, Atg21 furthermore covered the whole phagophore in some cells (chap. 4.2.2). This is in clear contrast to starvation conditions, where Atg21 never covered the phagophore. This indicated that there might be differences in the role of Atg21 in phagophore assembly dependent on the analyzed growth conditions. The expanded distribution of Atg21 at the phagophore either suggests that Atg8 lipidation is no longer restricted to the vacuolephagophore contact site or that Atg21 exhibits an additional function at the phagophore which could explain its importance under nutrient rich conditions (stationary cells) compared to starvation conditions.

The main difference in PAS formation under nutrient rich compared to starvation conditions is the requirement for Atg11 as a scaffold to recruit the core autophagic machinery. ATG11 deletion leads to a defect in the Cvt-pathway while under starvation conditions pApe1 maturation is only partially defective (Kim et al., 2001; Shintani and Klionsky, 2004; Suzuki et al., 2007). Apart from the Cvt-pathway Atg11 is important for a variety of selective types of autophagy (Kim et al., 2001; Kanki and Klionsky, 2008). Under starvation conditions the Atg17-Atg31-Atg29 complex exhibits the scaffolding function at the PAS and is together with Atg11 required for the transport of pApe1 to the vacuole (Kabeya et al., 2005; Cheong et al., 2005; Kuninori Suzuki et al., 2007; Kawamata et al., 2008). However, Atg17 is dispensable for the Cvt-pathway indicating that there are differences in the organization of the PAS depending on the growth conditions (Kabeya et al., 2005; Cheong et al., 2005). This might also include the recruitment of the Atg8 lipidation complex. A joint function of Atg11 and Atg21 could explain why Atg21 and Atg11 are both especially important for selective autophagy and could lead to the additional localization of Atg21 at the whole phagophore.

Remarkably, Atg17 and Atg11 display distinct localization patterns at GACs. While Atg17 localizes to a single dot at the vacuole-phagophore contact site, Atg11 covers the whole or at least parts of the GAC (Suzuki et al., 2013; Pfaffenwimmer et al., 
2014). This localization of Atg11 is probably mediated by its interaction with Atg19 the cargo receptor of the Cvt-pathway. A joint function or a direct interaction of Atg21 and Atg11 under nutrient rich conditions could therefore change the localization of Atg21. However, so far there are no hints for a direct interaction of Atg21 and Atg11. This will need further investigation.

Interestingly, S. pombe Atg18a which seems to have a function in Atg8 lipidation was observed to cover the whole enlarged cup-shaped structures that are generated in the absence of Ctl1 (chap. 5.2.1; Sun et al., 2013). This corresponds to the elongated localization of Atg21 in stationary cells. It suggests that either Atg8 lipidation is not restricted to a specific site at the phagophore or that both proteins share an additional function. In S. cerevisiae, this additional function would be only essential under nutrient rich conditions. In contrast, the Atg5 orthologue from Arabidopsis thaliana was observed to reside at the rim of the growing phagophore (Le Bars et al., 2014), a localization similar to that of Atg18. All together this indicates that the exact mechanisms that determine the site of Atg8 lipidation are not yet fully understood and may differ among different species.

Remarkably, the aberrant localization of Atg21-YFP in stationary cells compared to starved cells was also observed in cells treated with rapamycin. The antifungal antibiotic rapamycin is an inhibitor of TORC1 and can therefore induce autophagy (Vézina et al., 1975; Barbet et al., 1996; Noda and Ohsumi, 1998). The inhibition of TORC1 leads to a rapid dephosphorylation of Atg13 which initiates the assembly of the Atg1 kinase complex and subsequently the generation of autophagosomes (Kamada et al., 2000; Kamada et al., 2010). Since TORC1 is the primary sensor of nitrogen, its inhibition with rapamycin should therefore simulate nitrogen starvation conditions (Noda and Ohsumi, 1998). Accordingly, it is surprising that Atg21 displayed a different localization in rapamycin treated cells compared to nitrogen-starved cells.

For the induction of autophagy rapamycin was directly added to stationary cells while for nitrogen starvation the cells were transferred to fresh SD-N medium. SD-N medium supplies the cells with glucose as a carbon source but starves them for amino acids and ammonium sulfate (Takeshige et al., 1992). In contrast, the rapamycin treated stationary cells have consumed most of the glucose and other 
nutrients in their medium. This might lead to the induction of autophagy not only by rapamycin but also by glucose starvation which could explain the observed differences between nitrogen-starved and rapamycin-treated cells. In contrast to nitrogen starvation, a recent study showed that under glucose starvation autophagy is highly dependent on both scaffold proteins, Atg17 and Atg11 (Adachi et al., 2017). As proposed above, the requirement for Atg11 under glucose starvation could influence the localization of Atg21. For further investigation the rapamycin-based experiments should be repeated with logarithmic cells which are supplied with sufficient glucose to exclude glucose starvation.

\subsubsection{Identification of a novel vacuole-phagophore contact site}

The phagophore is frequently reported to be adjacent to the vacuole as well as the ER (Suzuki et al., 2013; Graef et al., 2013; Gomez-Sanchez et al., 2018). Recent studies revealed that this subcellular positioning of the phagophore coincides with a functional contact site with the ER (Gomez-Sanchez et al., 2018; Kotani et al., 2018). Corresponding to these findings, this study revealed the formation of a functional contact site between vacuole and phagophore (chap. 4.2.5 and 4.2.6). This contact site seems to be independent of the ER-phagophore contact site since the ER was not observed to be in proximity of the vacuole-phagophore contact site (4.2.6.4).

The vacuole-phagophore contact site is dependent on the vacuolar membrane protein Vac8 (chap. 4.2.5; Wang et al., 1998; Pan and Goldfarb, 1998; Fleckenstein et al., 1998). In the absence of Vac8, the contact of the PAS/phagophore with the vacuolar membrane was significantly reduced. Thereby, the PAS formation was not affected suggesting that the contact site is not required for the initiation of autophagy. However, the contact site seems to be essential for the elongation of the phagophore, since phagophore formation was significantly reduced in a vac80 strain (chap 4.2.5.2). Vac8 is essential for the progression of the Cvt-pathway but not for unselective bulk autophagy (Wang et al., 1998; Scott et al., 2000), indicating that the formation of the vacuole-phagophore contact site is especially required for the Cvtpathway. Furthermore, Vac8 is reported to interact with Atg13 (Scott et al., 2000; Jeong et al., 2017), suggesting that the vacuole-phagophore contact site is established by the association of both proteins. 
Remarkably, Vac8 is also involved in the formation of another vacuolar contact site with the nuclear ER, called NVJ. Here, the contact between the two membranes is established by a direct interaction between Vac8 (vacuolar membrane) and Nvj1 (nuclear ER) (Pan et al., 2000). A recent study reported that Nvj1 as well as Atg13 interact with Vac8 via the same positively charged residues of Vac8 and thereby compete for Vac8 binding (Jeong et al., 2017). This is in line with the findings of this study, that the vacuole-phagophore contact site is formed without any contact to the NVJ and therefore seems to be independent of it (chap. 4.2.5.1 and 4.2.6.1). The mutation of the positively charged residues of Vac8 inhibits the formation of the NVJ and further disrupts the progression of the Cvt-pathway (Jeong et al., 2017). This supports the hypothesis that the vacuole-phagophore contact site is established by the direct interaction of Vac8 and Atg13.

Apart from its participation in the NVJ, Vac8 is a key player in vacuole inheritance and regulates homotypic vacuole fusion (Wang et al., 1998; Pan and Goldfarb, 1998; Pan et al., 2000). The localization and function of Vac8 at the vacuolar membrane is dependent on its palmitoylation which seems to be a regulated process (Wang et al., 2001; Dietrich et al., 2005; Subramanian et al., 2006; Peng et al., 2006). The palmitoylation of Vac8 is mediated by the R-SNARE Ykt6 (Dietrich et al., 2005). Interestingly, Ykt6 is required for the fusion of autophagosomes with the vacuole and was identified as a potential interactor of Atg21 in this study (chap. 4.3.2; Bas et al., 2018; Gao et al., 2018b). During the homotypic vacuole fusion reaction, Vac8 is palmitoylated. It is thought to act after the Rab GTPase dependent tethering of the two membranes as well as the formation of the trans-SNARE complex (Wang et al., 2001). This could suggest that Vac8 and the vacuole-phagophore contact site might have a role in autophagosome-vacuole fusion.

The localization of Atg21 at the phagophore was observed to be in close proximity of the vacuole-phagophore contact site suggesting that Atg21 might contribute to the establishment or maintenance of the contact site (chap. 4.2.6.3). This would correspond to the second proposed model for membrane binding of the Atg21Atg16 complex, where it could be involved in the tethering of the vacuolar and autophagic membrane (chap. 5.1.2). Furthermore, Atg21 was observed to be more important for PAS formation while the vacuole-phagophore contact site is required for phagophore elongation (chap. 4.2.5.2). Therefore, Atg21 seems to act prior to 
Vac8 in autophagosome biogenesis which could indicate that Atg21 mediates the establishment of the vacuole-phagophore contact site. However, the deletion of ATG21 had no effect on the contact between vacuole and phagophore (chap. 4.2.5.3). Therefore, Atg21 does not seem to be a key component of the contact site between vacuole and phagophore which however does not exclude its participation in it. GFP-TRAP experiments revealed that Atg21 and Vac8 are at least part of the same complex or do even directly interact with each other (chap. 4.2.5.4). This interaction could be a reason for the restricted localization of Atg21 to the vacuole-phagophore contact site.

The vacuole-phagophore contact site can be further characterized by the specific exclusion of Vph1 (chap. 4.2.6.1) while Pho8 and FM4-64 are evenly distributed (chap. 4.2.6.2). These findings correlate with the observed characteristics of the NVJ (Dawaliby and Mayer, 2010). Considering the fact that both contact sites are dependent on Vac8 this suggests that they are formed and maintained by similar or the same mechanisms (Pan et al., 2000).

The vacuolar multi-spanning transmembrane protein $\mathrm{Vph} 1$ is a subunit of the $\mathrm{V}_{0}$ domain of the vacuolar V-ATPase complex (Manolson et al., 1992; Parra et al., 2014). The V-ATPase is an ATP-driven proton pump which is responsible for the acidification of the vacuolar lumen. It comprises the membrane intrinsic $\mathrm{V}_{0}$ domain consisting of six subunits and the peripheral $V_{1}$ domain which consists of eight subunits (Parra et al., 2014). It could be assumed that the exclusion of Vph1 from the NVJ and the vacuole-phagophore contact site is a result of steric hindrance due to the size of the large multi-subunit V-ATPase complex. However, Dawaliby et al. (2010) reported that the vacuolar transporter chaperon complex, which has a similar size as the V-ATPase complex, is not excluded from the NVJ. This indicates that the formation of the contact sites does not coincide with a simple size exclusion. There seem to be other mechanisms that selectively exclude Vph1.

Interestingly, Vph1 is reported to be excluded from most of the contact sites and membrane domains that are formed at the vacuolar membrane during various conditions (Tsuji and Fujimoto, 2018). Toulmay and Prinz (2013) observed the formation of large $L_{o}$ domains in the vacuolar membrane of late stationary yeast cells ( 2 days). These Lo domains are enriched for sterols suggesting that they 
represent lipid rafts. Furthermore, they are enriched for the proteins Ivy1 and Gtr2 while Vph1 as well as FM4-64 are excluded. This indicates that Vph1 might be a specific marker for $\mathrm{Ld}_{d}$ domains while Ivy1 and Gtr2 might be markers for Lo domains. The exclusion of Vph1 from the vacuole-phagophore contact site could therefore coincide with the formation of a lipid raft ( $L_{0}$ domain) at the vacuolar membrane. It is reported that palmitoylation increases the affinity of proteins for lipid rafts (Simons and Sampaio, 2011). This suggests that Vac8 could be specifically targeted to the vacuole-phagophore contact site via its palmitoylation (Peng et al., 2006). However, a direct contribution of lipids to the specialized membrane domain at the vacuolar membrane could not be demonstrated in this study. FM4-64, which is excluded from the Lo domains in late stationary cells (Toulmay and Prinz, 2013), was almost evenly distributed at the vacuole-phagophore contact site. This corresponds to the observation that FM4-64 is not excluded from NVJs although sterols and sphingolipids seem to be important for their formation (Dawaliby and Mayer, 2010). Therefore, more experiments are required to further characterize the vacuole-phagophore contact site.

FM4-64 staining of the vacuolar membrane further revealed that there is no direct membrane contact between the vacuole and the phagophore that could result into a direct flux of lipids into the phagophore (chap. 4.2.6.2). Interestingly, NVJs and vacuole-mitochondria patches, two membrane contact sites, are reported to be involved in non-vesicular lipid transport. The transfer of ergosterol to the vacuolar membrane is thought to be mediated by the ER-resident protein Ltc1 which is enriched in NVJs and vacuole-mitochondria patches (Murley et al., 2015). Therefore, a similar protein-mediated transport of lipids from the vacuole to the phagophore could be possible.

\subsection{The potential interactome of Atg21}

An essential breakthrough in understanding the function of a protein in the cell is the identification of its interaction partners. This is especially important when analyzing the function of scaffold proteins such as the PROPPIN-family which do not harbor an intrinsic enzymatic activity (Krick et al., 2012; Baskaran et al., 2012; Watanabe et al., 2012; Scacioc et al., 2017). Although PROPPINs are structurally well characterized, most of their functions remain elusive. They localize to various 
organelles in the cell (endosomes, vacuole or autophagic membrane) suggesting that they exhibit a variety of functions (Dove et al., 2004; Stromhaug et al., 2004; Meiling-Wesse et al., 2004; Krick et al., 2008a). Nevertheless, very little is known about the interactome of PROPPINs. This study focused on the identification of interaction partners of Atg21 to further elucidate its role in cellular processes.

Prior to this study a variety of approaches including recombinant pull down assays as well as co-immunoprecipitations followed by MS analysis were used to identify interacting proteins of Atg21. In gel filtration chromatography Atg21 was found to be part of a high molecular weight complex. However, this complex was very instable and sensitive to the applied buffer conditions suggesting that Atg21containing complexes might exhibit only weak or transient interactions. In consequence, none of the used approaches resulted in the identification of promising interactors of Atg21 (Juris (2014) and personal communication of Dr. Roswitha Krick). Independent of these attempts, so far only Atg16 and Atg8 could be identified as interacting proteins of Atg21 (Juris et al., 2015).

To circumvent the isolation of the instable Atg21 complexes in their native conformation this study made use of an in vivo labeling approach, namely the BioID assay, to identify potential interactors of Atg21.

\subsubsection{The BiolD assay}

In the last 10 years, a set of techniques has evolved which permit a proximity-based labeling of proteins in living cells. These techniques can be grouped into two categories: biotin ligase-based methods and peroxidase-based methods. All techniques involve the in vivo biotinylation of proteins in a proximity-dependent manner. The main advantage of these methods is that they do not require the preservation of protein complexes during lysis and affinity purification. Thereby, the identification of weak and transient interactions is enabled (Kim and Roux, 2016).

The BioID assay was first described by Roux et al. (2013). It belongs to the biotin ligase-based methods and is extensively described in chapter 4.3. Peroxide-based methods, as indicated by their name, use the enzymatic activity of peroxidases to generate short-lived radicals which react with electron rich side chains of amino 
acids (Chen and Perrimon, 2017). One of the main approaches among the peroxidebased methods was first described by Rhee et al. (2013) and makes use of engineered variants of the ascorbate peroxidase (APEX and APEX2) from plants (Rhee et al., 2013; Lam et al., 2015). Thereby, APEX2 represents an improved version of APEX with a higher activity and sensitivity. APEX and APEX2 convert their substrate biotin-phenol into a highly reactive and short-lived $(<1 \mathrm{~ms})$ biotinphenoxyl radical. The reaction is activated by the addition of $\mathrm{H}_{2} \mathrm{O}_{2}$ and can be stopped by its removal. The biotin-phenoxyl radical covalently conjugates to nearby electron rich amino acid side chains, namely tyrosines, and thereby labels the respective proteins with biotin (Rhee et al., 2013; Lam et al., 2015; Chen and Perrimon, 2017). Biotinylated proteins can be isolated and identified similar to the BioID assay (chap. 4.3).

BirA* and APEX2 are estimated to have labeling radii of about 10-20 nm. However, the short-lived $(<1 \mathrm{~ms}$ ) biotin-phenoxyl radicals generated by APEX2 are thought to result in a shorter labeling radius compared to the long-lived ( $>1 \mathrm{~min})$ biotinoyl-5'-AMP produced by BirA*. Remarkably, APEX2 has much faster kinetics compared to BirA*. Using APEX2 an efficient labeling with biotin can be achieved within 1 min after the activation with $\mathrm{H}_{2} \mathrm{O}_{2}$ while $\mathrm{BirA}^{*}$ requires labeling times of 15-24 h. Therefore, APEX2 may have a higher spatial and temporal labeling specificity than BirA*. Furthermore, the E. coli-derived BirA* has a temperature optimum at $37^{\circ} \mathrm{C}$. Its activity is highly reduced below the optimal temperature while APEX2 was reported to work efficiently at $25^{\circ} \mathrm{C}$ as well as $37^{\circ} \mathrm{C}$. Accordingly, the APEX-based assay seems to be the favored method for the proximity-dependent labeling in yeast cells with a growth optimum at $30^{\circ} \mathrm{C}$. However, the adaptation of both, the BioID and the APEX2-based assay, for the use in S. cerevisiae revealed one main drawback of the APEX2-based assay (Hwang and Espenshade, 2016; Opitz et al., 2017). While biotin, the substrate of BirA*, is actively transported to the cytosol of the cell and therefore readily available for the biotinylation reaction, biotinphenol can only inefficiently pass the cell wall of yeast cells (J Stolz et al., 1999; Hwang and Espenshade, 2016). To achieve an efficient labeling of proteins with APEX2, the yeast cells need to be treated with zymolyase in combination with high osmotic solutions (1.2 M sorbitol) to remove their cell walls and therefore enable the uptake of biotin-phenol (Hwang and Espenshade, 2016). Furthermore, the 
requirement of $\mathrm{H}_{2} \mathrm{O}_{2}$ for the activation of the labeling reaction by APEX2 represents another stress factor for the cell which could affect the cellular organization of protein complexes. However, due to the short labeling time (1 $\mathrm{min}$ ) the effect of the $\mathrm{H}_{2} \mathrm{O}_{2}$ on the cells might not be too drastic.

Opitz et al. (2017) reported that BirA* efficiently biotinylates proteins in yeast cells although not working at its optimal temperature. They used the BioID assay to evaluate the interactome of Asc1, a WD40-repeat scaffold protein at the head region of the 40S ribosomal subunit (Opitz et al., 2017). Thereby, it was proven that the BioID assay is suitable to investigate interacting proteins of WD40-repeat proteins making it the assay of choice for this study.

\subsubsection{A potential role of Atg21 in the fusion of autophagosomes with the vacuole}

In the first performed SILAC-based BioID experiment in this study 18 proteins were identified to be especially enriched in the BirA*-Atg21 derived light sample (chap. 4.3.2). The most abundant among these proteins comprising 6 biotin sites was the bait Atg21 itself. In combination with the specific biotinylation of Atg16 by BirA*Atg21 (chap. 4.3.1.3), this indicated that BirA*-Atg21 is potent to biotinylate interacting and proximal proteins and further that the SILAC-based approach was working. Therefore, the remaining 17 identified proteins could be considered as potential interaction partners of Atg21 or at least as potential components of Atg21containing complexes.

Most of the 17 identified potential interactors of Atg21 were classified as membrane associated proteins including vacuolar and endosomal membranes as well as cytosolic vesicles. This correlates with the reported localization of Atg21 in the cell (Figure 7.5; Stromhaug et al., 2004; Meiling-Wesse et al., 2004; Krick et al., 2008a). Functionally, more than half of the proteins are involved in intracellular transport processes which are mainly vesicle-mediated, including endosomal or Golgi-vesicle transport. This corresponds to the reported function of Atg21 in the Cvt-pathway and its localization to the PAS and endosomes (Barth et al., 2002; Krick et al., 2008a; Juris et al., 2015). Due to the clustering of potential interactors of Atg21 in vesicle- 
mediated transport, these proteins were assumed as most probable and interesting interactors of Atg21 that could probably elucidate the role of Atg21 at endosomes.

Among these proteins, Ykt6 participates in the fusion of autophagosomes with the vacuole and thereby affects autophagy progression (Meiling-Wesse et al., 2002; Bas et al., 2018; Gao et al., 2018b). The R-SNARE Ykt6 is involved in a variety of vesicle mediated transport pathways in the cell including trafficking to and within the Golgi, endocytic trafficking as well as trafficking to the vacuole (McNew et al., 1997; Kweon et al., 2003). It was recently identified as the autophagosomal SNARE in autophagosome-vacuole fusion. Thereby, it forms a trans-SNARE complex with the vacuolar Q-SNAREs Vam3, Vam7 and Vti1 which further facilitates the fusion event (Bas et al., 2018; Gao et al., 2018b). The potential interaction of Atg21 and Ykt6 could be required for the proper targeting of Ykt6 to the autophagic membrane. Further experiments are required to confirm the interaction of Atg21 with Ykt6, however, their interaction would suggest a functional link between Atg21 and the autophagosome-vacuole fusion.

Interestingly, at least 4 of the 17 potential interactors of Atg21 including Ykt6 are palmitoylated proteins (Roth et al. 2006). Vac8 which is also a potential interactor of Atg21 is palmitoylated as well (chap. 4.2.5.4; Wang et al., 2001; Dietrich et al., 2005). This seems to be a common feature of Atg21 interactors and corresponds to its localization to the vacuole-phagophore contact site. The formation of a lipid raft at the contact site between vacuole and phagophore might be the reason for the recruitment of the palmitoylated proteins (Simons and Sampaio, 2011). This could enable their interaction with Atg21.

All together the reported functions of the potential interactors, similar to their cellular localization, highly correspond to the known localization of Atg21 in the cell (Stromhaug et al., 2004; Meiling-Wesse et al., 2004; Krick et al., 2008a). Although further experiments are required to verify the interaction of Atg21 with the identified proteins, they provide a promising basis for the elucidation of the function of Atg21 in the cell. These findings further support that the BioID assay is a powerful tool to investigate the composition of target protein-containing complexes. In following experiments, the SILAC-based BioID assay could be used to investigate potential differences in the proteins interacting with Atg21 depending on the 
growth condition, including starved cells versus growing cells. Furthermore, Atg14 and VPS38 deletions could be used to specifically release Atg21 from its PAS or endosomal pool and to investigate the resulting differences in the interacting proteins of Atg21. This could help to distinguish between the endosomal and the autophagic function of Atg21.

\subsection{Conclusion}

This study revealed that the growing phagophore forms a functional contact site with the vacuolar membrane. This contact site is dependent on the vacuolar membrane protein Vac8 and coincides with the formation of a specialized domain at the vacuolar membrane which is able to specifically exclude Vph1. The vacuolephagophore contact site is involved in the elongation of the phagophore and independent of the ER-phagophore contact site as well as the NVJ. Atg21 restricts the Atg8 lipidation to the established vacuole-phagophore contact site. Thereby, the interaction of Atg21 and the Atg12 Atg5/Atg16 complex is mediated by the formation of a single salt bridge between Atg21 and Atg16. Furthermore, it can be proposed that Atg21 has an additional function in the autophagosome-vacuole fusion due to its potential interaction with Ykt6 which is a component of the vacuolar fusion machinery. 


\section{Bibliography}

Abreu, S., Kriegenburg, F., Gomez-Sanchez, R., Mari, M., Sanchez-Wandelmer, J., Skytte Rasmussen, M., et al. (2017) Conserved Atg8 recognition sites mediate Atg4 association with autophagosomal membranes and Atg8 deconjugation. EMBO Rep 18: $765-780$.

Adachi, A., Koizumi, M., and Ohsumi, Y. (2017) Autophagy induction under carbon starvation conditions is negatively regulated by carbon catabolite repression. Journal of Biological Chemistry 292: 19905-19918.

Alemu, E.A., Lamark, T., Torgersen, K.M., Birgisdottir, A.B., Larsen, K.B., Jain, A., et al. (2012) ATG8 family proteins act as scaffolds for assembly of the ULK complex: sequence requirements for LC3-interacting region (LIR) motifs. Journal of Biological Chemistry 287: 39275-39290.

Araki, Y., Ku, W.-C., Akioka, M., May, A.I., Hayashi, Y., Arisaka, F., et al. (2013) Atg38 is required for autophagy-specific phosphatidylinositol 3-kinase complex integrity. J Cell Biol 203: 299-313.

Ashford, T.P., and Porter, K.R. (1962) Cytoplasmic components in hepatic cell lysosomes. J Cell Biol 12: 198-202.

Baba, M., Osumi, M., and Ohsumi, Y. (1995) Analysis of the membrane structures involved in autophagy in yeast by freeze-replica method. Cell Struct Funct 20: 465471.

Baba, M., Osumi, M., Scott, S.V., Klionsky, D.J., and Ohsumi, Y. (1997) Two distinct pathways for targeting proteins from the cytoplasm to the vacuole/lysosome. J Cell Biol 139: 1687-1695.

Baba, M., Takeshige, K., Baba, N., and Ohsumi, Y. (1994) Ultrastructural analysis of the autophagic process in yeast: detection of autophagosomes and their characterization. J Cell Biol 124: 903-913.

Baba, M., Tomonaga, S., Suzuki, M., Gen, M., Takeda, E., Matsuura, A., et al. (2018) A nuclear membrane-derived structure associated with Atg8 is involved in the sequestration of selective cargo, the Cvt complex, during autophagosome formation in yeast. Autophagy 1-15.

Bacia, K., and Schwille, P. (2003) A dynamic view of cellular processes by in vivo fluorescence auto- and cross-correlation spectroscopy. Methods 29: 74-85.

Bacia, K., Kim, S.A., and Schwille, P. (2006) Fluorescence cross-correlation spectroscopy in living cells. Nat Methods 3: 83-89.

Backues, S.K., Orban, D.P., Bernard, A., Singh, K., Cao, Y., and Klionsky, D.J. (2015) Atg23 and Atg27 Act at the Early Stages of Atg9 Trafficking in S. cerevisiae. Traffic 16: $172-190$. 
Barbet, N.C., Schneider, U., Helliwell, S.B., Stansfield, I., Tuite, M.F., and Hall, M.N. (1996) TOR controls translation initiation and early G1 progression in yeast. Mol Biol Cell 7: 25-42.

Barth, H., Meiling-Wesse, K., Epple, U.D., and Thumm, M. (2001) Autophagy and the cytoplasm to vacuole targeting pathway both require Aut10p. FEBS Letters 508: 23-28.

Barth, H., Meiling-Wesse, K., Epple, U.D., and Thumm, M. (2002) Mai1p is essential for maturation of proaminopeptidase I but not for autophagy. FEBS Letters 512: 173-179.

Bas, L., Papinski, D., Licheva, M., Torggler, R., Rohringer, S., Schuschnig, M., and Kraft, C. (2018) Reconstitution reveals Ykt6 as the autophagosomal SNARE in autophagosome-vacuole fusion. J Cell Biol 217: 3656-3669.

Baskaran, S., Ragusa, M.J., Boura, E., and Hurley, J.H. (2012) Two-site recognition of phosphatidylinositol 3-phosphate by PROPPINs in autophagy. Mol Cell 47: 339348.

Bertani, G. (1951) Studies on lysogenesis. I. The mode of phage liberation by lysogenic Escherichia coli. J Bacteriol 62: 293-300.

Budovskaya, Y., Stephan, J., Deminoff, S., and Herman, P. (2005) An evolutionary proteomics approach identifies substrates of the cAMP-dependent protein kinase. Proc Natl Acad Sci U S A 102: 13933-13938.

Budovskaya, Y., Stephan, J., Reggiori, F., Klionsky, D., and Herman, P. (2004) The Ras/cAMP-dependent protein kinase signaling pathway regulates an early step of the autophagy process in Saccharomyces cerevisiae. J Biol Chem 279: 2066320671.

Burda, P., Padilla, S., Sarkar, S., and Emr, S. (2002) Retromer function in endosometo-Golgi retrograde transport is regulated by the yeast Vps34 PtdIns 3-kinase. J Cell Sci 115: 3889-3900.

Busse, R.A., Scacioc, A., Krick, R., Perez-Lara, A., Thumm, M., and Kühnel, K. (2015) Characterization of PROPPIN-Phosphoinositide Binding and Role of Loop 6CD in PROPPIN-Membrane Binding. Biophys J 108: 2223-2234.

Carlsson, S.R., and Simonsen, A. (2015) Membrane dynamics in autophagosome biogenesis. J Cell Sci 128: 193-205.

Cebollero, E., der Vaart, van, A., Zhao, M., Rieter, E., Klionsky, D.J., Helms, J.B., and Reggiori, F. (2012) Phosphatidylinositol-3-phosphate clearance plays a key role in autophagosome completion. Curr Biol 22: 1545-1553.

Chapman-Smith, A., and Cronan, J.E. (1999) Molecular biology of biotin attachment to proteins. J Nutr 129: 477S-484S. 
Chen, C.-L., and Perrimon, N. (2017) Proximity-dependent labeling methods for proteomic profiling in living cells. Wiley Interdiscip Rev Dev Biol 6: e272.

Cheng, J., Fujita, A., Yamamoto, H., Tatematsu, T., Kakuta, S., Obara, K., et al. (2014) Yeast and mammalian autophagosomes exhibit distinct phosphatidylinositol 3phosphate asymmetries. Nat Commun 5: 3207.

Cheong, H., Nair, U., Geng, J., and Klionsky, D.J. (2008) The Atg1 kinase complex is involved in the regulation of protein recruitment to initiate sequestering vesicle formation for nonspecific autophagy in Saccharomyces cerevisiae. Mol Biol Cell 19: 668-681.

Cheong, H., Yorimitsu, T., Reggiori, F., Legakis, J., Wang, C., and Klionsky, D. (2005) Atg17 regulates the magnitude of the autophagic response. Mol Biol Cell 16: 34383453.

Chew, L.H., Setiaputra, D., Klionsky, D.J., and Yip, C.K. (2013) Structural characterization of the Saccharomyces cerevisiae autophagy regulatory complex Atg17-Atg31-Atg29. Autophagy 9.

Choi-Rhee, E., Schulman, H., and Cronan, J.E. (2004) Promiscuous protein biotinylation by Escherichia coli biotin protein ligase. Protein Sci 13: 3043-3050.

CLARK, S.L. (1957) Cellular differentiation in the kidneys of newborn mice studies with the electron microscope. J Biophys Biochem Cytol 3: 349-362.

Cox, J., and Mann, M. (2008) MaxQuant enables high peptide identification rates, individualized p.p.b.-range mass accuracies and proteome-wide protein quantification. Nature Biotechnology 26: 1367-1372.

Cronan, J.E. (2005) Targeted and proximity-dependent promiscuous protein biotinylation by a mutant Escherichia coli biotin protein ligase. J Nutr Biochem 16: 416-418.

Cuervo, A.M., and Wong, E. (2014) Chaperone-mediated autophagy: roles in disease and aging. Cell Res 24: 92-104.

Darsow, T., Rieder, S., and Emr, S. (1997) A multispecificity syntaxin homologue, Vam3p, essential for autophagic and biosynthetic protein transport to the vacuole. J Cell Biol 138: 517-529.

Davis, S., and Ferro-Novick, S. (2015) Ypt1 and COPII vesicles act in autophagosome biogenesis and the early secretory pathway. Biochem Soc Trans 43: 92-96.

Dawaliby, R., and Mayer, A. (2010) Microautophagy of the nucleus coincides with a vacuolar diffusion barrier at nuclear-vacuolar junctions. Mol Biol Cell 21: 41734183. 
De Craene, J.-O., Bertazzi, D.L., Bär, S., and Friant, S. (2017) Phosphoinositides, Major Actors in Membrane Trafficking and Lipid Signaling Pathways. Int J Mol Sci 18: 634 .

Dietrich, L.E.P., LaGrassa, T.J., Rohde, J., Cristodero, M., Meiringer, C.T.A., and Ungermann, C. (2005) ATP-independent control of Vac8 palmitoylation by a SNARE subcomplex on yeast vacuoles. J Biol Chem 280: 15348-15355.

Dilcher, M., Köhler, B., and Mollard, von, G.F. (2001) Genetic interactions with the yeast Q-SNARE VTI1 reveal novel functions for the R-SNARE YKT6.J Biol Chem 276: 34537-34544.

Dooley, H.C., Razi, M., Polson, H.E.J., Girardin, S.E., Wilson, M.I., and Tooze, S.A. (2014) WIPI2 Links LC3 Conjugation with PI3P, Autophagosome Formation, and Pathogen Clearance by Recruiting Atg12-5-16L1. Mol Cell 55: 238-252.

Dove, S., Piper, R., McEwen, R., Yu, J., King, M., Hughes, D., et al. (2004) Svp1p defines a family of phosphatidylinositol 3,5-bisphosphate effectors. EMBO J 23 : 1922-1933.

Dubouloz, F., Deloche, O., Wanke, V., Cameroni, E., and De Virgilio, C. (2005) The TOR and EGO protein complexes orchestrate microautophagy in yeast. Mol Cell 19: 15-26.

Duina, A.A., Miller, M.E., and Keeney, J.B. (2014) Budding yeast for budding geneticists: a primer on the Saccharomyces cerevisiae model system. Genetics 197: 33-48.

Dunn, W.A. (1990) Studies on the mechanisms of autophagy: maturation of the autophagic vacuole. J Cell Biol 110: 1935-1945.

Efe, J., Botelho, R., and Emr, S. (2007) Atg18 regulates organelle morphology and Fab1 kinase activity independent of its membrane recruitment by phosphatidylinositol 3,5-bisphosphate. Mol Biol Cell 18: 4232-4244.

Fader, C.M., Sánchez, D.G., Mestre, M.B., and Colombo, M.I. (2009) TI-VAMP/VAMP7 and VAMP3/cellubrevin: two v-SNARE proteins involved in specific steps of the autophagy/multivesicular body pathways. Biochim Biophys Acta 1793: 19011916.

Farré, J.-C., and Subramani, S. (2004) Peroxisome turnover by micropexophagy: an autophagy-related process. Trends Cell Biol 14: 515-523.

Farré, J.-C., and Subramani, S. (2016) Mechanistic insights into selective autophagy pathways: lessons from yeast. Nat Rev Mol Cell Biol 17: 537-552.

Farré, J.-C., Burkenroad, A., Burnett, S.F., and Subramani, S. (2013) Phosphorylation of mitophagy and pexophagy receptors coordinates their interaction with Atg8 and Atg11. EMBO Rep. 
Farré, J.-C., Manjithaya, R., Mathewson, R.D., and Subramani, S. (2008) PpAtg30 tags peroxisomes for turnover by selective autophagy. Dev Cell 14: 365-376.

Fischer von Mollard, G., and Stevens, T.H. (1999) The Saccharomyces cerevisiae vSNARE Vti1p is required for multiple membrane transport pathways to the vacuole. Mol Biol Cell 10: 1719-1732.

Fleckenstein, D., Rohde, M., Klionsky, D., and Rudiger, M. (1998) Yel013p (Vac8p), an armadillo repeat protein related to plakoglobin and importin alpha is associated with the yeast vacuole membrane. J Cell Sci 111 ( Pt 20): 3109-3118.

Fracchiolla, D., Sawa-Makarska, J., Zens, B., Ruiter, A. de, Zaffagnini, G., Brezovich, A., et al. (2016) Mechanism of cargo-directed Atg8 conjugation during selective autophagy. Elife 5: 18799.

Fujioka, Y., Noda, N.N., Nakatogawa, H., Ohsumi, Y., and Inagaki, F. (2010) Dimeric coiled-coil structure of Saccharomyces cerevisiae Atg16 and its functional significance in autophagy. Journal of Biological Chemistry 285: 1508-1515.

Fujioka, Y., Suzuki, S.W., Yamamoto, H., Kondo-Kakuta, C., Kimura, Y., Hirano, H., et al. (2014) Structural basis of starvation-induced assembly of the autophagy initiation complex. Nat Struct Mol Biol 1-12.

Fujita, N., Morita, E., Itoh, T., Tanaka, A., Nakaoka, M., Osada, Y., et al. (2013) Recruitment of the autophagic machinery to endosomes during infection is mediated by ubiquitin. J Cell Biol 203: 115-128.

Fujita, N., Saitoh, T., Kageyama, S., Akira, S., Noda, T., and Yoshimori, T. (2009) Differential involvement of Atg16L1 in Crohn disease and canonical autophagy: analysis of the organization of the Atg16L1 complex in fibroblasts. Journal of Biological Chemistry 284: 32602-32609.

Gao, J., Langemeyer, L., Kümmel, D., Reggiori, F., and Ungermann, C. (2018a) Molecular mechanism to target the endosomal Mon1-Ccz1 GEF complex to the preautophagosomal structure. Elife 7: 765.

Gao, J., Reggiori, F., and Ungermann, C. (2018b) A novel in vitro assay reveals SNARE topology and the role of Ykt6 in autophagosome fusion with vacuoles. J Cell Biol 217: 3670-3682.

Ge, L., Baskaran, S., Schekman, R., and Hurley, J.H. (2014) The protein-vesicle network of autophagy. Curr Opin Cell Biol 29C: 18-24.

Geng, J., Baba, M., Nair, U., and Klionsky, D.J. (2008) Quantitative analysis of autophagy-related protein stoichiometry by fluorescence microscopy. J Cell Biol 182: $129-140$.

Geng, J., Nair, U., Yasumura-Yorimitsu, K., and Klionsky, D.J. (2010) Post-Golgi Sec proteins are required for autophagy in Saccharomyces cerevisiae. Mol Biol Cell 21: 2257-2269. 
Goffeau, A., Barrell, B., Bussey, H., Davis, R., Dujon, B., Feldmann, H., et al. (1996) Life with 6000 genes. Science 274: 546.

Gomez-Sanchez, R., Rose, J., Guimarães, R., Mari, M., Papinski, D., Rieter, E., et al. (2018) Atg9 establishes Atg2-dependent contact sites between the endoplasmic reticulum and phagophores. J Cell Biol 217: 2743-2763.

González Bardeci, N., Angiolini, J.F., De Rossi, M.C., Bruno, L., and Levi, V. (2017) Dynamics of intracellular processes in live-cell systems unveiled by fluorescence correlation microscopy. IUBMB Life 69: 8-15.

Goody, R.S., Müller, M.P., and Wu, Y.-W. (2017) Mechanisms of action of Rab proteins, key regulators of intracellular vesicular transport. Biol Chem 398: 565575.

Gopaldass, N., Fauvet, B., Lashuel, H., Roux, A., and Mayer, A. (2017) Membrane scission driven by the PROPPIN Atg18. EMBO J 36: 3274-3291.

Graef, M. (2018) Lipid droplet-mediated lipid and protein homeostasis in budding yeast. FEBS Lett 592: 1291-1303.

Graef, M., Friedman, J.R., Graham, C., Babu, M., and Nunnari, J. (2013) ER exit sites are physical and functional core autophagosome biogenesis components. Mol Biol Cell 24: 2918-2931.

Guan, J., Stromhaug, P., George, M., Habibzadegah-Tari, P., Bevan, A., Dunn, W., and Klionsky, D. (2001) Cvt18/Gsa12 is required for cytoplasm-to-vacuole transport, pexophagy, and autophagy in Saccharomyces cerevisiae and Pichia pastoris. Mol Biol Cell 12: 3821-3838.

Hailey, D.W., Rambold, A.S., Satpute-Krishnan, P., Mitra, K., Sougrat, R., Kim, P.K., and Lippincott-Schwartz, J. (2010) Mitochondria supply membranes for autophagosome biogenesis during starvation. Cell 141: 656-667.

Hamasaki, M., Noda, T., Baba, M., and Ohsumi, Y. (2005) Starvation triggers the delivery of the endoplasmic reticulum to the vacuole via autophagy in yeast. Traffic 6: $56-65$.

Hanada, T., Noda, N.N., Satomi, Y., Ichimura, Y., Fujioka, Y., Takao, T., et al. (2007) The Atg12-Atg5 conjugate has a novel E3-like activity for protein lipidation in autophagy. J Biol Chem 282: 37298-37302.

Hanahan, D. (1983) Studies on transformation of Escherichia coli with plasmids. J Mol Biol 166: 557-580.

Hanahan, D. (1985) Techniques for transformation of E. coli. DNA cloning: a practical approach 1: 109-135.

Harding, T., Morano, K., Scott, S., and Klionsky, D. (1995) Isolation and characterization of yeast mutants in the cytoplasm to vacuole protein targeting pathway. J Cell Biol 131: 591-602. 
Harding, T.M., Hefner-Gravink, A., Thumm, M., and Klionsky, D.J. (1996) Genetic and phenotypic overlap between autophagy and the cytoplasm to vacuole protein targeting pathway. J Biol Chem 271: 17621-17624.

He, C., Baba, M., Cao, Y., and Klionsky, D.J. (2008) Self-interaction is critical for Atg9 transport and function at the phagophore assembly site during autophagy. Mol Biol Cell 19: 5506-5516.

Helms, J.B., and Zurzolo, C. (2004) Lipids as targeting signals: lipid rafts and intracellular trafficking. Traffic 5: 247-254.

Herman, P., Stack, J., and Emr, S. (1991) A genetic and structural analysis of the yeast Vps15 protein kinase: evidence for a direct role of Vps15p in vacuolar protein delivery. EMBO J 10: 4049-4060.

Hickey, C.M., and Wickner, W. (2010) HOPS initiates vacuole docking by tethering membranes before trans-SNARE complex assembly. Mol Biol Cell 21: 2297-2305.

Ho, R., and Stroupe, C. (2015) The HOPS/class C Vps complex tethers membranes by binding to one Rab GTPase in each apposed membrane. Mol Biol Cell 26: 26552663.

Hong, S.B., Kim, B.-W., Lee, K.-E., Kim, S.W., Jeon, H., Kim, J., and Song, H.K. (2011) Insights into noncanonical E1 enzyme activation from the structure of autophagic E1 Atg7 with Atg8. Nat Struct Mol Biol 18: 1323-1330.

Hosokawa, N., Hara, T., Kaizuka, T., Kishi, C., Takamura, A., Miura, Y., et al. (2009) Nutrient-dependent mTORC1 Association with the ULK1-Atg13-FIP200 Complex Required for Autophagy. Mol Biol Cell.

Hurley, J.H., and Young, L.N. (2017) Mechanisms of Autophagy Initiation. Annu Rev Biochem 86: 225-244.

Hutchins, M., and Klionsky, D. (2001) Vacuolar localization of oligomeric alphamannosidase requires the cytoplasm to vacuole targeting and autophagy pathway components in Saccharomyces cerevisiae. J Biol Chem 276: 20491-20498.

Hwang, J., and Espenshade, P.J. (2016) Proximity-dependent biotin labelling in yeast using the engineered ascorbate peroxidase APEX2. Biochem J 473: 24632469.

Ichimura, Y., Imamura, Y., Emoto, K., Umeda, M., Noda, T., and Ohsumi, Y. (2004) In vivo and in vitro reconstitution of Atg8 conjugation essential for autophagy. J Biol Chem 279: 40584-40592.

Ichimura, Y., Kirisako, T., Takao, T., Satomi, Y., Shimonishi, Y., Ishihara, N., et al. (2000) A ubiquitin-like system mediates protein lipidation. Nature 408: 488-492.

Ishihara, N., Hamasaki, M., Yokota, S., Suzuki, K., Kamada, Y., Kihara, A., et al. (2001) Autophagosome requires specific early Sec proteins for its formation and NSF/SNARE for vacuolar fusion. Mol Biol Cell 12: 3690-3702. 
Jain, B.P., and Pandey, S. (2018) WD40 Repeat Proteins: Signalling Scaffold with Diverse Functions. Protein J 37: 391-406.

Janke, C., Magiera, M., Rathfelder, N., Taxis, C., Reber, S., Maekawa, H., et al. (2004) A versatile toolbox for PCR-based tagging of yeast genes: new fluorescent proteins, more markers and promoter substitution cassettes. Yeast 21: 947-962.

Jao, C.C., Ragusa, M.J., Stanley, R.E., and Hurley, J.H. (2013) A HORMA domain in Atg13 mediates PI 3-kinase recruitment in autophagy. Proc Natl Acad Sci U S A 110: 5486-5491.

Jeffries, T., Dove, S., Michell, R., and Parker, P. (2004) PtdIns-specific MPR pathway association of a novel WD40 repeat protein, WIPI49. Mol Biol Cell 15: 2652-2663.

Jeong, H., Park, J., Kim, H.-I., Lee, M., Ko, Y.-J., Lee, S., et al. (2017) Mechanistic insight into the nucleus-vacuole junction based on the Vac8p-Nvj1p crystal structure. Proc Natl Acad Sci US A 114: E4539-E4548.

Jin, N., Chow, C.Y., Liu, L., Zolov, S.N., Bronson, R., Davisson, M., et al. (2008) VAC14 nucleates a protein complex essential for the acute interconversion of PI3P and $\mathrm{PI}(3,5) \mathrm{P}(2)$ in yeast and mouse. EMBO J 27: 3221-3234.

Johnsson, N., and Varshavsky, A. (1994) Split ubiquitin as a sensor of protein interactions in vivo. Proc Natl Acad Sci USA 91: 10340-10344.

Juris, L. (2014) Atg21 functions during autophagy as a scaffold for the E3 ubiquitin-like complex in Atg8 lipidation. 1-163.

Juris, L., Montino, M., Rube, P., Schlotterhose, P., Thumm, M., and Krick, R. (2015) PI3P binding by Atg21 organises Atg8 lipidation. EMBO J 34: 955-973.

Kabeya, Y., Kamada, Y., Baba, M., Takikawa, H., Sasaki, M., and Ohsumi, Y. (2005) Atg17 functions in cooperation with Atg1 and Atg13 in yeast autophagy. Mol Biol Cell 16: 2544-2553.

Kabeya, Y., Kawamata, T., Suzuki, K., and Ohsumi, Y. (2007) Cis1/Atg31 is required for autophagosome formation in Saccharomyces cerevisiae. Biochem Biophys Res Commun 356: 405-410.

Kabeya, Y., Noda, N.N., Fujioka, Y., Suzuki, K., Inagaki, F., and Ohsumi, Y. (2009) Characterization of the Atg17-Atg29-Atg31 complex specifically required for starvation-induced autophagy in Saccharomyces cerevisiae. Biochem Biophys Res Commun 389: 612-615.

Kageyama, T., Suzuki, K., and Ohsumi, Y. (2009) Lap3 is a selective target of autophagy in yeast, Saccharomyces cerevisiae. Biochem Biophys Res Commun 378: 551-557.

Kaiser, C., Michaelis, S., and Mitchell, A. (1994) Methods in Yeast Genetics, pp: 137147. 
Kamada, Y., Funakoshi, T., Shintani, T., Nagano, K., Ohsumi, M., and Ohsumi, Y. (2000) Tor-mediated induction of autophagy via an Apg1 protein kinase complex. J Cell Biol 150: 1507-1513.

Kamada, Y., Yoshino, K.-I., Kondo, C., Kawamata, T., Oshiro, N., Yonezawa, K., and Ohsumi, Y. (2010) Tor directly controls the Atg1 kinase complex to regulate autophagy. Mol Cell Biol 30: 1049-1058.

Kanki, T., and Klionsky, D.J. (2008) Mitophagy in yeast occurs through a selective mechanism. J Biol Chem 283: 32386-32393.

Kanki, T., Furukawa, K., and Yamashita, S.-I. (2015) Mitophagy in yeast: Molecular mechanisms and physiological role. BBA - Molecular Cell Research 1853: 27562765.

Kanki, T., Wang, K., Baba, M., Bartholomew, C.R., Lynch-Day, M.A., Du, Z., et al. (2009a) A genomic screen for yeast mutants defective in selective mitochondria autophagy. Mol Biol Cell 20: 4730-4738.

Kanki, T., Wang, K., Cao, Y., Baba, M., and Klionsky, D.J. (2009b) Atg32 is a mitochondrial protein that confers selectivity during mitophagy. Dev Cell 17: 98109.

Kaufmann, A., and Wollert, T. (2014) Scaffolding the expansion of autophagosomes. Autophagy 10: 1343-1345.

Kaufmann, A., Beier, V., Franquelim, H.G., and Wollert, T. (2014) Molecular mechanism of autophagic membrane-scaffold assembly and disassembly. Cell 156: 469-481.

Kawamata, T., Kamada, Y., Kabeya, Y., Sekito, T., and Ohsumi, Y. (2008) Organization of the pre-autophagosomal structure responsible for autophagosome formation. Mol Biol Cell 19: 2039-2050.

Kihara, A., Noda, T., Ishihara, N., and Ohsumi, Y. (2001) Two distinct Vps34 phosphatidylinositol 3-kinase complexes function in autophagy and carboxypeptidase Y sorting in Saccharomyces cerevisiae. J Cell Biol 152: 519-530.

Kim, D.I., and Roux, K.J. (2016) Filling the Void: Proximity-Based Labeling of Proteins in Living Cells. Trends Cell Biol 26: 804-817.

Kim, D.I., Birendra, K.C., Zhu, W., Motamedchaboki, K., Doye, V., and Roux, K.J. (2014) Probing nuclear pore complex architecture with proximity-dependent biotinylation. Proc Natl Acad Sci U S A 111: E2453-61.

Kim, J., Kamada, Y., Stromhaug, P.E., Guan, J., Hefner-Gravink, A., Baba, M., et al. (2001) Cvt9/Gsa9 functions in sequestering selective cytosolic cargo destined for the vacuole. J Cell Biol 153: 381-396. 
Kim, J., Scott, S., Oda, M., and Klionsky, D. (1997) Transport of a large oligomeric protein by the cytoplasm to vacuole protein targeting pathway. J Cell Biol 137: 609-618.

Kirisako, T., Baba, M., Ishihara, N., Miyazawa, K., Ohsumi, M., Yoshimori, T., et al. (1999) Formation process of autophagosome is traced with Apg8/Aut7p in yeast. J Cell Biol 147: 435-446.

Kirisako, T., Ichimura, Y., Okada, H., Kabeya, Y., Mizushima, N., Yoshimori, T., et al. (2000) The reversible modification regulates the membrane-binding state of Apg8/Aut7 essential for autophagy and the cytoplasm to vacuole targeting pathway. J Cell Biol 151: 263-276.

Klionsky, D., and Emr, S. (1989) Membrane protein sorting: biosynthesis, transport and processing of yeast vacuolar alkaline phosphatase. EMBO J 8: 2241-2250.

Klionsky, D., Cueva, R., and Yaver, D. (1992) Aminopeptidase I of Saccharomyces cerevisiae is localized to the vacuole independent of the secretory pathway. J Cell Biol 119: 287-299.

Knop, M., Siegers, K., Pereira, G., Zachariae, W., Winsor, B., Nasmyth, K., and Schiebel, E. (1999) Epitope tagging of yeast genes using a PCR-based strategy: more tags and improved practical routines. Yeast 15: 963-972.

Kofinger, J., Ragusa, M.J., Lee, I.-H., Hummer, G., and Hurley, J.H. (2015) Solution structure of the Atg1 complex: implications for the architecture of the phagophore assembly site. Structure 23: 809-818.

Komatsu, M., Tanida, I., Ueno, T., Ohsumi, M., Ohsumi, Y., and Kominami, E. (2001) The C-terminal region of an Apg7p/Cvt2p is required for homodimerization and is essential for its E1 activity and E1-E2 complex formation. J Biol Chem 276: 98469854.

Kotani, T., Kirisako, H., Koizumi, M., Ohsumi, Y., and Nakatogawa, H. (2018) The Atg2-Atg18 complex tethers pre-autophagosomal membranes to the endoplasmic reticulum for autophagosome formation. Proc Natl Acad Sci U S A 115: 1036310368.

Kozlov, M.M., Campelo, F., Liska, N., Chernomordik, L.V., Marrink, S.J., and McMahon, H.T. (2014) Mechanisms shaping cell membranes. Curr Opin Cell Biol 29: 53-60.

Kraft, C., Deplazes, A., Sohrmann, M., and Peter, M. (2008) Mature ribosomes are selectively degraded upon starvation by an autophagy pathway requiring the Ubp3p/Bre5p ubiquitin protease. Nat Cell Biol 10: 602-610.

Kraft, C., Kijanska, M., Kalie, E., Siergiejuk, E., Lee, S.S., Semplicio, G., et al. (2012) Binding of the Atg1/ULK1 kinase to the ubiquitin-like protein Atg8 regulates autophagy. EMBO J 31: 3691-3703. 
Krämer, L., and Ungermann, C. (2011) HOPS drives vacuole fusion by binding the vacuolar SNARE complex and the Vam7 PX domain via two distinct sites. Mol Biol Cell 22: 2601-2611.

Krick, R., and Thumm, M. (2016) Atg8 lipidation is coordinated in a PtdIns3Pdependent manner by the PROPPIN Atg21. Autophagy 12: 2260-2261.

Krick, R., Busse, R.A., Scacioc, A., Stephan, M., Janshoff, A., Thumm, M., and Kühnel, K. (2012) Structural and functional characterization of the two phosphoinositide binding sites of PROPPINs, a $\beta$-propeller protein family. Proc Natl Acad Sci U S A 109: E2042-9.

Krick, R., Henke, S., Tolstrup, J., and Thumm, M. (2008a) Dissecting the localization and function of Atg18, Atg21 and Ygr223c. Autophagy 4: 896-910.

Krick, R., Muehe, Y., Prick, T., Bremer, S., Schlotterhose, P., Eskelinen, E.-L., et al. (2008b) Piecemeal microautophagy of the nucleus requires the core macroautophagy genes. Mol Biol Cell 19: 4492-4505.

Krick, R., Tolstrup, J., Appelles, A., Henke, S., and Thumm, M. (2006) The relevance of the phosphatidylinositolphosphat-binding motif FRRGT of Atg18 and Atg21 for the Cvt pathway and autophagy. FEBS Letters 580: 4632-4638.

Kuma, A., Mizushima, N., Ishihara, N., and Ohsumi, Y. (2002) Formation of the approximately 350-kDa Apg12-Apg5.Apg16 multimeric complex, mediated by Apg16 oligomerization, is essential for autophagy in yeast. J Biol Chem 277: 18619-18625.

Kumar, S., and Nussinov, R. (2002) Close-range electrostatic interactions in proteins. ChemBioChem 3: 604-617.

Kumeta, H., Watanabe, M., Nakatogawa, H., Yamaguchi, M., Ogura, K., Adachi, W., et al. (2010) The NMR structure of the autophagy-related protein Atg8. J Biomol NMR 47: 237-241.

Kutateladze, T.G. (2010) Translation of the phosphoinositide code by PI effectors. Nat Chem Biol 6: 507-513.

Kweon, Y., Rothe, A., Conibear, E., and Stevens, T.H. (2003) Ykt6p is a multifunctional yeast R-SNARE that is required for multiple membrane transport pathways to the vacuole. Mol Biol Cell 14: 1868-1881.

Lam, S.S., Martell, J.D., Kamer, K.J., Deerinck, T.J., Ellisman, M.H., Mootha, V.K., and Ting, A.Y. (2015) Directed evolution of APEX2 for electron microscopy and proximity labeling. Nat Methods 12: 51-54.

Lane, M.D., Young, D.L., and Lynen, F. (1964) The enzymatic synthesis of holotranscarboxylase from apotranscarboxylase and (+)-biotin. I. Purification of the apoenzyme and synthetase; characteristics of the reaction.J Biol Chem 239: 2858-2864. 
Lang, T., Reiche, S., Straub, M., Bredschneider, M., and Thumm, M. (2000) Autophagy and the cvt pathway both depend on AUT9. J Bacteriol 182: 21252133.

Lang, T., Schaeffeler, E., Bernreuther, D., Bredschneider, M., Wolf, D.H., and Thumm, M. (1998) Aut2p and Aut7p, two novel microtubule-associated proteins are essential for delivery of autophagic vesicles to the vacuole. EMBO J 17: 3597-3607.

Lämmli, U. (1970) Cleavage of structural proteins during the assembly of the head of Bacteriophage T4. Nature 227: 680-685.

Le Bars, R., Marion, J., Le Borgne, R., Satiat-Jeunemaitre, B., and Bianchi, M.W. (2014) ATG5 defines a phagophore domain connected to the endoplasmic reticulum during autophagosome formation in plants. Nat Commun 5: 4121.

Leão-Helder, A.N., Krikken, A.M., Gellissen, G., der Klei, van, I.J., Veenhuis, M., and Kiel, J.A.K.W. (2004) Atg21p is essential for macropexophagy and microautophagy in the yeast Hansenula polymorpha. FEBS Letters 577: 491-495.

Leber, R., Silles, E., Sandoval, I., and Mazon, M. (2001) Yol082p, a novel CVT protein involved in the selective targeting of aminopeptidase I to the yeast vacuole. J Biol Chem 276: 29210-29217.

Li, W.-W., Li, J., and Bao, J.-K. (2012) Microautophagy: lesser-known self-eating. Cell Mol Life Sci 69: 1125-1136.

Liu, X., and Klionsky, D.J. (2016) The Atg17-Atg31-Atg29 complex and Atg11 regulate autophagosome-vacuole fusion. Autophagy 12: 894-895.

Longtine, M., McKenzie, A., Demarini, D., Shah, N., Wach, A., Brachat, A., et al. (1998) Additional modules for versatile and economical PCR-based gene deletion and modification in Saccharomyces cerevisiae. Yeast 14: 953-961.

Lynch-Day, M.A., Bhandari, D., Menon, S., Huang, J., Cai, H., Bartholomew, C.R., et al. (2010) Trs85 directs a Ypt1 GEF, TRAPPIII, to the phagophore to promote autophagy. Proc Natl Acad Sci U S A 107: 7811-7816.

Manolson, M., Proteau, D., Preston, R., Stenbit, A., Roberts, B., Hoyt, M., et al. (1992) The VPH1 gene encodes a 95-kDa integral membrane polypeptide required for in vivo assembly and activity of the yeast vacuolar $\mathrm{H}(+)$-ATPase. J Biol Chem 267: 14294-14303.

Mao, K., Chew, L.H., Inoue-Aono, Y., Cheong, H., Nair, U., Popelka, H., et al. (2013) Atg29 phosphorylation regulates coordination of the Atg17-Atg31-Atg29 complex with the Atg11 scaffold during autophagy initiation. Proc Natl Acad Sci U S A 110: E2875-E2884.

Mari, M., Griffith, J., Rieter, E., Krishnappa, L., Klionsky, D.J., and Reggiori, F. (2010) An Atg9-containing compartment that functions in the early steps of autophagosome biogenesis. J Cell Biol 190: 1005-1022. 
McNew, J.A., Sogaard, M., Lampen, N.M., Machida, S., Ye, R.R., Lacomis, L., et al. (1997) Ykt6p, a prenylated SNARE essential for endoplasmic reticulum-Golgi transport. J Biol Chem 272: 17776-17783.

Meiling-Wesse, K., Barth, H., Voss, C., Barmark, G., Murén, E., Ronne, H., and Thumm, M. (2002) Yeast Mon1p/Aut12p functions in vacuolar fusion of autophagosomes and cvt-vesicles. FEBS Letters 530: 174-180.

Meiling-Wesse, K., Barth, H., Voss, C., Eskelinen, E.-L., Epple, U.D., and Thumm, M. (2004) Atg21 is required for effective recruitment of Atg8 to the preautophagosomal structure during the Cvt pathway. J Biol Chem 279: 3774137750 .

Meiling-Wesse, K., Epple, U.D., Krick, R., Barth, H., Appelles, A., Voss, C., et al. (2005) Trs85 (Gsg1), a component of the TRAPP complexes, is required for the organization of the preautophagosomal structure during selective autophagy via the Cvt pathway. J Biol Chem 280: 33669-33678.

Metje, J. (2017) Structural characterization of autophagy related protein complexes. 1-187.

Mizushima, N., Kuma, A., Kobayashi, Y., Yamamoto, A., Matsubae, M., Takao, T., et al. (2003) Mouse Apg16L, a novel WD-repeat protein, targets to the autophagic isolation membrane with the Apg12-Apg5 conjugate. J Cell Sci 116: 1679-1688.

Mizushima, N., Levine, B., Cuervo, A.M., and Klionsky, D.J. (2008) Autophagy fights disease through cellular self-digestion. Nature 451: 1069-1075.

Mizushima, N., Noda, T., and Ohsumi, Y. (1999) Apg16p is required for the function of the Apg12p-Apg5p conjugate in the yeast autophagy pathway. EMBO J 18: 38883896.

Mizushima, N., Noda, T., Yoshimori, T., Tanaka, Y., Ishii, T., George, M.D., et al. (1998) A protein conjugation system essential for autophagy. Nature 395: 395398.

Mizushima, N., Yamamoto, A., Hatano, M., Kobayashi, Y., Kabeya, Y., Suzuki, K., et al. (2001) Dissection of autophagosome formation using Apg5-deficient mouse embryonic stem cells. J Cell Biol 152: 657-668.

Mizushima, N., Yoshimori, T., and Ohsumi, Y. (2011) The role of atg proteins in autophagosome formation. Annu Rev Cell Dev Biol 27: 107-132.

Mochida, K., Oikawa, Y., Kimura, Y., Kirisako, H., Hirano, H., Ohsumi, Y., and Nakatogawa, H. (2015) Receptor-mediated selective autophagy degrades the endoplasmic reticulum and the nucleus. Nature 522: 359-362.

Mohrlüder, J., Hoffmann, Y., Stangler, T., Hänel, K., and Willbold, D. (2007) Identification of clathrin heavy chain as a direct interaction partner for the gammaaminobutyric acid type A receptor associated protein. Biochemistry 46: 1453714543. 
Mortimore, G.E., and Schworer, C.M. (1977) Induction of autophagy by amino-acid deprivation in perfused rat liver. Nature 270: 174-176.

Motley, A.M., Nuttall, J.M., and Hettema, E.H. (2012) Pex3-anchored Atg36 tags peroxisomes for degradation in Saccharomyces cerevisiae. EMBO J 31: 2852-2868.

Mumberg, D., Muller, R., and Funk, M. (1994) Regulatable promoters of Saccharomyces cerevisiae: comparison of transcriptional activity and their use for heterologous expression. Nucleic Acids Res 22: 5767-5768.

Murley, A., Sarsam, R.D., Toulmay, A., Yamada, J., Prinz, W.A., and Nunnari, J. (2015) Ltc1 is an ER-localized sterol transporter and a component of ER-mitochondria and ER-vacuole contacts. J Cell Biol 209: 539-548.

Nair, U., Cao, Y., Xie, Z., and Klionsky, D.J. (2010) Roles of the lipid-binding motifs of Atg18 and Atg21 in the cytoplasm to vacuole targeting pathway and autophagy. Journal of Biological Chemistry 285: 11476-11488.

Nair, U., Yen, W.-L., Mari, M., Cao, Y., Xie, Z., Baba, M., et al. (2012) A role for Atg8PE deconjugation in autophagosome biogenesis. Autophagy 8: 780-793.

Nakatogawa, H., Ichimura, Y., and Ohsumi, Y. (2007) Atg8, a ubiquitin-like protein required for autophagosome formation, mediates membrane tethering and hemifusion. Cell 130: 165-178.

Nakatogawa, H., Ishii, J., Asai, E., and Ohsumi, Y. (2012a) Atg4 recycles inappropriately lipidated Atg8 to promote autophagosome biogenesis. Autophagy 8: 177-186.

Nakatogawa, H., Ohbayashi, S., Sakoh-Nakatogawa, M., Kakuta, S., Suzuki, S.W., Kirisako, H., et al. (2012b) The Autophagy-related Protein Kinase Atg1 Interacts with the Ubiquitin-like Protein Atg8 via the Atg8 Family Interacting Motif to Facilitate Autophagosome Formation. Journal of Biological Chemistry 287: 2850328507.

Nakatogawa, H., Suzuki, K., Kamada, Y., and Ohsumi, Y. (2009) Dynamics and diversity in autophagy mechanisms: lessons from yeast. Nat Rev Mol Cell Biol 10: 458-467.

Nath, S., Dancourt, J., Shteyn, V., Puente, G., Fong, W.M., Nag, S., et al. (2014) Lipidation of the LC3/GABARAP family of autophagy proteins relies on a membrane-curvature-sensing domain in Atg3. Nat Cell Biol 16: 415-424.

Neiman, A.M. (2005) Ascospore formation in the yeast Saccharomyces cerevisiae. Microbiol Mol Biol Rev 69: 565-584.

Ngu, M.P., Hirata, E., and Suzuki, K. (2015) Visualization of Atg3 during Autophagosome Formation in Saccharomyces cerevisiae. J Biol Chem 290: 81468153. 
Niedenthal, R., Riles, L., Johnston, M., and Hegemann, J. (1996) Green fluorescent protein as a marker for gene expression and subcellular localization in budding yeast. Yeast 12: 773-786.

Noda, N.N., and Fujioka, Y. (2015) Atg1 family kinases in autophagy initiation. Cell Mol Life Sci 72: 3083-3096.

Noda, N.N., Kumeta, H., Nakatogawa, H., Satoo, K., Adachi, W., Ishii, J., et al. (2008) Structural basis of target recognition by Atg8/LC3 during selective autophagy. Genes Cells 13: 1211-1218.

Noda, N.N., Ohsumi, Y., and Inagaki, F. (2010) Atg8-family interacting motif crucial for selective autophagy. FEBS Letters 584: 1379-1385.

Noda, N.N., Satoo, K., Fujioka, Y., Kumeta, H., Ogura, K., Nakatogawa, H., et al. (2011) Structural basis of Atg8 activation by a homodimeric E1, Atg7. Mol Cell 44: 462475.

Noda, T., and Ohsumi, Y. (1998) Tor, a phosphatidylinositol kinase homologue, controls autophagy in yeast. J Biol Chem 273: 3963-3966.

Noda, T., Kim, J., Huang, W.P., Baba, M., Tokunaga, C., Ohsumi, Y., and Klionsky, D.J. (2000) Apg9p/Cvt7p is an integral membrane protein required for transport vesicle formation in the Cvt and autophagy pathways. J Cell Biol 148: 465-480.

Nordmann, M., Cabrera, M., Perz, A., Bröcker, C., Ostrowicz, C., Engelbrecht-Vandré, S., and Ungermann, C. (2010) The Mon1-Ccz1 complex is the GEF of the late endosomal Rab7 homolog Ypt7. Curr Biol 20: 1654-1659.

Novikoff, A.B. (1959) The proximal tubule cell in experimental hydronephrosis. $J$ Biophys Biochem Cytol 6: 136-138.

Novikoff, A.B., and Essner, E. (1962) Cytolysomes and mitochondrial degeneration. J Cell Biol 15: 140-146.

Obara, K., Noda, T., Niimi, K., and Ohsumi, Y. (2008a) Transport of phosphatidylinositol 3-phosphate into the vacuole via autophagic membranes in Saccharomyces cerevisiae. Genes Cells 13: 537-547.

Obara, K., Sekito, T., and Ohsumi, Y. (2006) Assortment of phosphatidylinositol 3kinase complexes--Atg14p directs association of complex I to the preautophagosomal structure in Saccharomyces cerevisiae. Mol Biol Cell 17: 15271539.

Obara, K., Sekito, T., Niimi, K., and Ohsumi, Y. (2008b) The Atg18-Atg2 complex is recruited to autophagic membranes via phosphatidylinositol 3-phosphate and exerts an essential function. J Biol Chem 283: 23972-23980.

Ohashi, Y., and Munro, S. (2010) Membrane delivery to the yeast autophagosome from the Golgi-endosomal system. Mol Biol Cell 21: 3998-4008. 
Ohashi, Y., Soler, N., Garcia Ortegon, M., Zhang, L., Kirsten, M.L., Perisic, O., et al. (2016) Characterization of Atg38 and NRBF2, a fifth subunit of the autophagic Vps34/PIK3C3 complex. Autophagy 12: 2129-2144.

Okamoto, K., Kondo-Okamoto, N., and Ohsumi, Y. (2009) Mitochondria-anchored receptor Atg32 mediates degradation of mitochondria via selective autophagy. Dev Cell 17: 87-97.

Ong, S.-E., and Mann, M. (2006) A practical recipe for stable isotope labeling by amino acids in cell culture (SILAC). Nat Protoc 1: 2650-2660.

Opitz, N., Schmitt, K., Hofer-Pretz, V., Neumann, B., Krebber, H., Braus, G.H., and Valerius, O. (2017) Capturing the Asc1p/Receptor for Activated C Kinase 1 (RACK1) Microenvironment at the Head Region of the 40S Ribosome with Quantitative BioID in Yeast. Mol Cell Proteomics 16: 2199-2218.

Pan, X., and Goldfarb, D.S. (1998) YEB3/VAC8 encodes a myristylated armadillo protein of the Saccharomyces cerevisiae vacuolar membrane that functions in vacuole fusion and inheritance. J Cell Sci 111 ( Pt 15): 2137-2147.

Pan, X., Roberts, P., Chen, Y., Kvam, E., Shulga, N., Huang, K., et al. (2000) Nucleusvacuole junctions in Saccharomyces cerevisiae are formed through the direct interaction of Vac8p with Nvj1p. Mol Biol Cell 11: 2445-2457.

Papinski, D., and Kraft, C. (2014) Atg1 kinase organizes autophagosome formation by phosphorylating Atg9. Autophagy 10: 1338-1340.

Papinski, D., Schuschnig, M., Reiter, W., Wilhelm, L., Barnes, C.A., Majolica, A., et al. (2014) Early Steps in Autophagy Depend on Direct Phosphorylation of Atg9 by the Atg1 Kinase. Mol Cell.

Parra, K.J., Chan, C.-Y., and Chen, J. (2014) Saccharomyces cerevisiae Vacuolar H+ATPase Regulation by Disassembly and Reassembly: One Structure and Multiple Signals. Eukaryotic Cell 13: 706-714.

Parrish, W., Stefan, C., and Emr, S. (2004) Essential role for the myotubularinrelated phosphatase Ymr1p and the synaptojanin-like phosphatases Sjl2p and Sjl3p in regulation of phosphatidylinositol 3-phosphate in yeast. Mol Biol Cell 15: 3567-3579.

Partow, S., Siewers, V., Bjørn, S., Nielsen, J., and Maury, J. (2010) Characterization of different promoters for designing a new expression vector in Saccharomyces cerevisiae. Yeast 27: 955-964.

Parzych, K.R., Ariosa, A., Mari, M., and Klionsky, D.J. (2018) A newly characterized vacuolar serine carboxypeptidase, Atg42/Ybr139w, is required for normal vacuole function and the terminal steps of autophagy in the yeast Saccharomyces cerevisiae. Mol Biol Cell 29: 1089-1099.

Peng, Y., Tang, F., and Weisman, L. (2006) Palmitoylation plays a role in targeting Vac8p to specific membrane subdomains. Traffic 7: 1378-1387. 
Pfaffenwimmer, T., Reiter, W., Brach, T., Nogellova, V., Papinski, D., Schuschnig, M., et al. (2014) Hrr25 kinase promotes selective autophagy by phosphorylating the cargo receptor Atg19. EMBO Rep.

Pfeifer, U. (1977) Inhibition by insulin of the physiological autophagic breakdown of cell organelles. Acta Biol Med Ger 36: 1691-1694.

Pink, M., Verma, N., Rettenmeier, A.W., and Schmitz-Spanke, S. (2010) CBB staining protocol with higher sensitivity and mass spectrometric compatibility.

Electrophoresis 31: 593-598.

Pirner, H.M., and Stolz, J. (2006) Biotin sensing in Saccharomyces cerevisiae is mediated by a conserved DNA element and requires the activity of biotin-protein ligase. J Biol Chem 281: 12381-12389.

Polson, H.E.J., De Lartigue, J., Rigden, D.J., Reedijk, M., Urbé, S., Clague, M.J., and Tooze, S.A. (2010) Mammalian Atg18 (WIPI2) localizes to omegasome-anchored phagophores and positively regulates LC3 lipidation. Autophagy 6: 506-522.

Prinz, W., Grzyb, L., Veenhuis, M., Kahana, J., Silver, P., and Rapoport, T. (2000) Mutants affecting the structure of the cortical endoplasmic reticulum in Saccharomyces cerevisiae. J Cell Biol 150: 461-474.

Proikas-Cezanne, T., Takacs, Z., Donnes, P., and Kohlbacher, O. (2015) WIPI proteins: essential PtdIns3P effectors at the nascent autophagosome. J Cell Sci 128: 207-217.

Proikas-Cezanne, T., Waddell, S., Gaugel, A., Frickey, T., Lupas, A., and Nordheim, A. (2004) WIPI-1alpha (WIPI49), a member of the novel 7-bladed WIPI protein family, is aberrantly expressed in human cancer and is linked to starvationinduced autophagy. Oncogene 23: 9314-9325.

Puri, C., Renna, M., Bento, C.F., Moreau, K., and Rubinsztein, D.C. (2013) Diverse autophagosome membrane sources coalesce in recycling endosomes. Cell 154: 1285-1299.

Puri, C., Vicinanza, M., Ashkenazi, A., Gratian, M.J., Zhang, Q., Bento, C.F., et al. (2018) The RAB11A-Positive Compartment Is a Primary Platform for Autophagosome Assembly Mediated by WIPI2 Recognition of PI3P-RAB11A. Dev Cell 45: 114-131.e8.

Ragusa, M.J., Stanley, R.E., and Hurley, J.H. (2012) Architecture of the Atg17 Complex as a Scaffold for Autophagosome Biogenesis. Cell 151: 1501-1512.

Rao, Y., Perna, M.G., Hofmann, B., Beier, V., and Wollert, T. (2016) The Atg1-kinase complex tethers Atg9-vesicles to initiate autophagy. Nat Commun 7: 10338.

Ravikumar, B., Moreau, K., Jahreiss, L., Puri, C., and Rubinsztein, D.C. (2010) Plasma membrane contributes to the formation of pre-autophagosomal structures. Nat Cell Biol 12: 747-757. 
Reggiori, F., and Klionsky, D.J. (2013) Autophagic processes in yeast: mechanism, machinery and regulation. Genetics 194: 341-361.

Reggiori, F., and Ungermann, C. (2017) Autophagosome Maturation and Fusion. $J$ Mol Biol 429: 486-496.

Reggiori, F., Shintani, T., Nair, U., and Klionsky, D. (2005) Atg9 cycles between mitochondria and the pre-autophagosomal structure in yeasts. Autophagy 1: 101109.

Reggiori, F., Tucker, K., Stromhaug, P., and Klionsky, D. (2004) The Atg1-Atg13 complex regulates Atg9 and Atg23 retrieval transport from the preautophagosomal structure. Dev Cell 6: 79-90.

Reidick, C., Boutouja, F., and Platta, H.W. (2017) The class III phosphatidylinositol 3-kinase Vps34 in Saccharomyces cerevisiae. Biol Chem 398: 677-685.

Rhee, H.-W., Zou, P., Udeshi, N.D., Martell, J.D., Mootha, V.K., Carr, S.A., and Ting, A.Y. (2013) Proteomic mapping of mitochondria in living cells via spatially restricted enzymatic tagging. Science 339: 1328-1331.

Rieter, E., Vinke, F., Bakula, D., Cebollero, E., Ungermann, C., Proikas-Cezanne, T., and Reggiori, F. (2013) Atg18 function in autophagy is regulated by specific sites within its $\beta$-propeller. J Cell Sci 126: 593-604.

Roberts, P., Moshitch-Moshkovitz, S., Kvam, E., O'Toole, E., Winey, M., and Goldfarb, D. (2003) Piecemeal microautophagy of nucleus in Saccharomyces cerevisiae. Mol Biol Cell 14: 129-141.

Roberts, R., and Ktistakis, N.T. (2013) Omegasomes: PI3P platforms that manufacture autophagosomes. Essays Biochem 55: 17-27.

Romanov, J., Walczak, M., Ibiricu, I., Schüchner, S., Ogris, E., Kraft, C., and Martens, S. (2012) Mechanism and functions of membrane binding by the Atg5-Atg12/Atg16 complex during autophagosome formation. EMBO J 31: 4304-4317.

Rose, A.B., and Broach, J.R. (1990) Propagation and expression of cloned genes in yeast: 2-microns circle-based vectors. Methods Enzymol 185: 234-279.

Roth A.F., Wan J., Bailey A.O., Sun B., Kuchar J.A., Green W.N., Phinney B.S., Yates J.R. 3rd, Davis N.G. (2006) Global analysis of protein palmitoylation in yeast. Cell 125: 1003-1013.

Roux, K.J., Kim, D.I., Raida, M., and Burke, B. (2012) A promiscuous biotin ligase fusion protein identifies proximal and interacting proteins in mammalian cells. $J$ Cell Biol 196: 801-810.

Rutherford, J.C., and Bird, A.J. (2004) Metal-responsive transcription factors that regulate iron, zinc, and copper homeostasis in eukaryotic cells. Eukaryotic Cell 3: $1-13$. 
Saitsu, H., Nishimura, T., Muramatsu, K., Kodera, H., Kumada, S., Sugai, K., et al. (2013) De novo mutations in the autophagy gene WDR45 cause static encephalopathy of childhood with neurodegeneration in adulthood. Nat Genet 45: 445-9-449e1.

Sakoh-Nakatogawa, M., Kirisako, H., Nakatogawa, H., and Ohsumi, Y. (2015) Localization of Atg3 to autophagy-related membranes and its enhancement by the Atg8-family interacting motif to promote expansion of the membranes. FEBS Letters 589: 744-749.

Sakoh-Nakatogawa, M., Matoba, K., Asai, E., Kirisako, H., Ishii, J., Noda, N.N., et al. (2013) Atg12-Atg5 conjugate enhances E2 activity of Atg3 by rearranging its catalytic site. Nat Struct Mol Biol 20: 433-439.

Sanchez-Wandelmer, J., Kriegenburg, F., Rohringer, S., Schuschnig, M., GomezSanchez, R., Zens, B., et al. (2017) Atg4 proteolytic activity can be inhibited by Atg1 phosphorylation. Nat Commun 8: 295.

Sawa-Makarska, J., Abert, C., Romanov, J., Zens, B., Ibiricu, I., and Martens, S. (2014) Cargo binding to Atg19 unmasks additional Atg8 binding sites to mediate membrane-cargo apposition during selective autophagy. Nat Cell Biol 16: 425433.

Scacioc, A., Schmidt, C., Hofmann, T., Urlaub, H., Kühnel, K., and Perez-Lara, A. (2017) Structure based biophysical characterization of the PROPPIN Atg18 shows Atg18 oligomerization upon membrane binding. Sci Rep 7: 14008.

Schindelin, J., Arganda-Carreras, I., Frise, E., Kaynig, V., Longair, M., Pietzsch, T., et al. (2012) Fiji: an open-source platform for biological-image analysis. Nat Methods 9: 676-682.

Schu, P., Takegawa, K., Fry, M., Stack, J., Waterfield, M., and Emr, S. (1993) Phosphatidylinositol 3-kinase encoded by yeast VPS34 gene essential for protein sorting. Science 260: 88-91.

Schwarten, M., Stoldt, M., Mohrlüder, J., and Willbold, D. (2010) Solution structure of Atg8 reveals conformational polymorphism of the N-terminal domain. Biochem Biophys Res Commun 395: 426-431.

Scott, S., Guan, J., Hutchins, M., Kim, J., and Klionsky, D. (2001) Cvt19 is a receptor for the cytoplasm-to-vacuole targeting pathway. Mol Cell 7: 1131-1141.

Scott, S.V., Nice, D.C., Nau, J.J., Weisman, L.S., Kamada, Y., Keizer-Gunnink, I., et al. (2000) Apg13p and Vac8p are part of a complex of phosphoproteins that are required for cytoplasm to vacuole targeting. J Biol Chem 275: 25840-25849.

Sekito, T., Kawamata, T., Ichikawa, R., Suzuki, K., and Ohsumi, Y. (2009) Atg17 recruits Atg9 to organize the pre-autophagosomal structure. Genes Cells 14: 525538.

Sherman, F. (2002) Getting started with yeast. Methods Enzymol 350: 3-41. 
Shintani, T., and Klionsky, D. (2004) Cargo proteins facilitate the formation of transport vesicles in the cytoplasm to vacuole targeting pathway. J Biol Chem 279: 29889-29894.

Shintani, T., Huang, W., Stromhaug, P., and Klionsky, D. (2002) Mechanism of cargo selection in the cytoplasm to vacuole targeting pathway. Dev Cell 3: 825-837.

Shintani, T., Mizushima, N., Ogawa, Y., Matsuura, A., Noda, T., and Ohsumi, Y. (1999) Apg10p, a novel protein-conjugating enzyme essential for autophagy in yeast. EMBO J 18: 5234-5241.

Shintani, T., Suzuki, K., Kamada, Y., Noda, T., and Ohsumi, Y. (2001) Apg2p functions in autophagosome formation on the perivacuolar structure. J Biol Chem 276: 30452-30460.

Shirahama-Noda, K., Kira, S., Yoshimori, T., and Noda, T. (2013) TRAPPIII is responsible for vesicular transport from early endosomes to Golgi, facilitating Atg9 cycling in autophagy. J Cell Sci 126: 4963-4973.

Sikorski, R., and Hieter, P. (1989) A system of shuttle vectors and yeast host strains designed for efficient manipulation of DNA in Saccharomyces cerevisiae. Genetics 122: 19-27.

Simons, K., and Sampaio, J.L. (2011) Membrane organization and lipid rafts. Cold Spring Harb Perspect Biol 3: a004697-a004697.

Slobodkin, M.R., and Elazar, Z. (2013) The Atg8 family: multifunctional ubiquitinlike key regulators of autophagy. Essays Biochem 55: 51-64.

Stack, J., and Emr, S. (1994) Vps34p required for yeast vacuolar protein sorting is a multiple specificity kinase that exhibits both protein kinase and phosphatidylinositol-specific PI 3-kinase activities. J Biol Chem 269: 31552-31562.

Stack, J., De, W., Takegawa, K., and Emr, S. (1995) Vesicle-mediated protein transport: regulatory interactions between the Vps15 protein kinase and the Vps34 PtdIns 3-kinase essential for protein sorting to the vacuole in yeast. J Cell Biol 129: 321-334.

Stephan, J.S., Yeh, Y.-Y., Ramachandran, V., Deminoff, S.J., and Herman, P.K. (2009) The Tor and PKA signaling pathways independently target the Atg1/Atg13 protein kinase complex to control autophagy. Proc Natl Acad Sci U S A 106: 17049-17054.

Stirnimann, C.U., Petsalaki, E., Russell, R.B., and Müller, C.W. (2010) WD40 proteins propel cellular networks. Trends in biochemical sciences 35: 565-574.

Stolz, A., Ernst, A., and Dikic, I. (2014) Cargo recognition and trafficking in selective autophagy. Nat Cell Biol 16: 495-501.

Stolz, J., Hoja, U., Meier, S., Sauer, N., and Schweizer, E. (1999) Identification of the plasma membrane H+-biotin symporter of Saccharomyces cerevisiae by rescue of a fatty acid-auxotrophic mutant. J Biol Chem 274: 18741-18746. 
Straub, M., Bredschneider, M., and Thumm, M. (1997) AUT3, a serine/threonine kinase gene, is essential for autophagocytosis in Saccharomyces cerevisiae. $J$ Bacteriol 179: 3875-3883.

Stromhaug, P., Reggiori, F., Guan, J., Wang, C., and Klionsky, D. (2004) Atg21 is a phosphoinositide binding protein required for efficient lipidation and localization of Atg8 during uptake of aminopeptidase I by selective autophagy. Mol Biol Cell 15: 3553-3566.

Subramanian, K., Dietrich, L.E.P., Hou, H., LaGrassa, T.J., Meiringer, C.T.A., and Ungermann, C. (2006) Palmitoylation determines the function of Vac8 at the yeast vacuole. J Cell Sci 119: 2477-2485.

Sun, L.-L., Li, M., Suo, F., Liu, X.-M., Shen, E.-Z., Yang, B., et al. (2013) Global Analysis of Fission Yeast Mating Genes Reveals New Autophagy Factors. PLoS Genet 9: e1003715.

Suzuki, H., Osawa, T., Fujioka, Y., and Noda, N.N. (2016) Structural biology of the core autophagy machinery. Current Opinion in Structural Biology 43: 10-17.

Suzuki, K., Akioka, M., Kondo-Kakuta, C., Yamamoto, H., and Ohsumi, Y. (2013) Fine mapping of autophagy-related proteins during autophagosome formation in Saccharomyces cerevisiae. J Cell Sci 126: 2534-2544.

Suzuki, K., Kirisako, T., Kamada, Y., Mizushima, N., Noda, T., and Ohsumi, Y. (2001) The pre-autophagosomal structure organized by concerted functions of APG genes is essential for autophagosome formation. EMBO J 20: 5971-5981.

Suzuki, K., Kubota, Y., Sekito, T., and Ohsumi, Y. (2007) Hierarchy of Atg proteins in pre-autophagosomal structure organization. Genes Cells 12: 209-218.

Suzuki, K., Morimoto, M., Kondo, C., and Ohsumi, Y. (2011) Selective Autophagy Regulates Insertional Mutagenesis by the Ty1 Retrotransposon in Saccharomyces cerevisiae. Developmental Cell 21: 358-365.

Suzuki, K., Noda, T., and Ohsumi, Y. (2004) Interrelationships among Atg proteins during autophagy in Saccharomyces cerevisiae. Yeast 21: 1057-1065.

Suzuki, S.W., Yamamoto, H., Oikawa, Y., Kondo-Kakuta, C., Kimura, Y., Hirano, H., and Ohsumi, Y. (2015) Atg13 HORMA domain recruits Atg9 vesicles during autophagosome formation. Proc Natl Acad Sci USA 112: 3350-3355.

Takeshige, K., Baba, M., Tsuboi, S., Noda, T., and Ohsumi, Y. (1992) Autophagy in yeast demonstrated with proteinase-deficient mutants and conditions for its induction. J Cell Biol 119: 301-311.

Tamura, N., Oku, M., and Sakai, Y. (2014) Atg21 regulates pexophagy via its PI(3)Pbinding activity in Pichia pastoris. FEMS Yeast Res 14: 435-444. 
Tamura, N., Oku, M., Ito, M., Noda, N.N., Inagaki, F., and Sakai, Y. (2013) Atg18 phosphoregulation controls organellar dynamics by modulating its phosphoinositide-binding activity. J Cell Biol 202: 685-698.

Tan, D., Cai, Y., Wang, J., Zhang, J., Menon, S., Chou, H.-T., et al. (2013) The EM structure of the TRAPPIII complex leads to the identification of a requirement for COPII vesicles on the macroautophagy pathway. Proc Natl Acad Sci U S A 110: 19432-19437.

Tang, B.L., Wang, Y., Ong, Y.S., and Hong, W. (2005) COPII and exit from the endoplasmic reticulum. Biochim Biophys Acta 1744: 293-303.

Tewari, R., Bailes, E., Bunting, K.A., and Coates, J.C. (2010) Armadillo-repeat protein functions: questions for little creatures. Trends Cell Biol 20: 470-481.

Thielmann, Y., Weiergräber, O.H., Mohrlüder, J., and Willbold, D. (2009) Structural framework of the GABARAP-calreticulin interface--implications for substrate binding to endoplasmic reticulum chaperones. FEBS J 276: 1140-1152.

Thumm, M., Busse, R.A., Scacioc, A., Stephan, M., Janshoff, A., Kühnel, K., and Krick, R. (2013) It takes two to tango: PROPPINs use two phosphoinositide-binding sites. Autophagy 9: 106-107.

Thumm, M., Egner, R., Koch, B., Schlumpberger, M., Straub, M., Veenhuis, M., and Wolf, D.H. (1994) Isolation of autophagocytosis mutants of Saccharomyces cerevisiae. FEBS Letters 349: 275-280.

Torggler, R., Papinski, D., and Kraft, C. (2017) Assays to Monitor Autophagy in Saccharomyces cerevisiae. Cells 6: 23.

Toulmay, A., and Prinz, W.A. (2013) Direct imaging reveals stable, micrometerscale lipid domains that segregate proteins in live cells. J Cell Biol 202: 35-44.

Tsuji, T., and Fujimoto, T. (2018) Lipids and lipid domains of the yeast vacuole. Biochem Soc Trans 46: 1047-1054.

Tsukada, M., and Ohsumi, Y. (1993) Isolation and characterization of autophagydefective mutants of Saccharomyces cerevisiae. FEBS Lett 333: 169-174.

Turkowyd, B., Virant, D., and Endesfelder, U. (2016) From single molecules to life: microscopy at the nanoscale. Anal Bioanal Chem 408: 6885-6911.

Varnaitè, R., and MacNeill, S.A. (2016) Meet the neighbors: Mapping local protein interactomes by proximity-dependent labeling with BioID. Proteomics 16: 25032518.

Vézina, C., Kudelski, A., and Sehgal, S.N. (1975) Rapamycin (AY-22,989), a new antifungal antibiotic. I. Taxonomy of the producing streptomycete and isolation of the active principle. J Antibiot 28: 721-726. 
Vida, T., and Emr, S. (1995) A new vital stain for visualizing vacuolar membrane dynamics and endocytosis in yeast. J Cell Biol 128: 779-792.

Wang, C., Stromhaug, P., Kauffman, E., Weisman, L., and Klionsky, D. (2003) Yeast homotypic vacuole fusion requires the Ccz1-Mon1 complex during the tethering/docking stage. J Cell Biol 163: 973-985.

Wang, C., Stromhaug, P., Shima, J., and Klionsky, D. (2002) The Ccz1-Mon1 protein complex is required for the late step of multiple vacuole delivery pathways. J Biol Chem 277: 47917-44727.

Wang, C.W., Kim, J., Huang, W.P., Abeliovich, H., Stromhaug, P.E., Dunn, W.A., and Klionsky, D.J. (2001) Apg2 is a novel protein required for the cytoplasm to vacuole targeting, autophagy, and pexophagy pathways. J Biol Chem 276: 30442-30451.

Wang, J., Menon, S., Yamasaki, A., Chou, H.-T., Walz, T., Jiang, Y., and Ferro-Novick, S. (2013) Ypt1 recruits the Atg1 kinase to the preautophagosomal structure. Proc Natl Acad Sci U S A 110: 9800-9805.

Wang, X., Li, X., and Li, Y. (2007) A modified Coomassie Brilliant Blue staining method at nanogram sensitivity compatible with proteomic analysis. Biotechnol Lett 29: 1599-1603.

Wang, Y.-X., Kauffman, E.J., Duex, J.E., and Weisman, L.S. (2001) Fusion of Docked Membranes Requires the Armadillo Repeat Protein Vac8p. J Biol Chem 276: 35133-35140.

Wang, Y.X., Catlett, N.L., and Weisman, L.S. (1998) Vac8p, a vacuolar protein with armadillo repeats, functions in both vacuole inheritance and protein targeting from the cytoplasm to vacuole. J Cell Biol 140: 1063-1074.

Watanabe, Y., Kobayashi, T., Yamamoto, H., Hoshida, H., Akada, R., Inagaki, F., et al. (2012) Structure-based analyses reveal distinct binding sites for Atg2 and phosphoinositides in Atg18. Journal of Biological Chemistry 287: 31681-31690.

Waterhouse, A.M., Procter, J.B., Martin, D.M.A., Clamp, M., and Barton, G.J. (2009) Jalview Version 2--a multiple sequence alignment editor and analysis workbench. Bioinformatics 25: 1189-1191.

Weidberg, H., Shpilka, T., Shvets, E., Abada, A., Shimron, F., and Elazar, Z. (2011a) LC3 and GATE-16 N Termini Mediate Membrane Fusion Processes Required for Autophagosome Biogenesis. Developmental Cell 20: 444-454.

Weidberg, H., Shvets, E., and Elazar, Z. (2011b) Biogenesis and cargo selectivity of autophagosomes. Annu Rev Biochem 80: 125-156.

Weidberg, H., Shvets, E., Shpilka, T., Shimron, F., Shinder, V., and Elazar, Z. (2010) LC3 and GATE-16/GABARAP subfamilies are both essential yet act differently in autophagosome biogenesis. EMBO J 29: 1792-1802. 
Wen, X., and Klionsky, D.J. (2016) An overview of macroautophagy in yeast. J Mol Biol.

Wickner, W., and Rizo, J. (2017) A cascade of multiple proteins and lipids catalyzes membrane fusion. Mol Biol Cell 28: 707-711.

Wilson, M.I., Dooley, H.C., and Tooze, S.A. (2014) WIPI2b and Atg16L1: setting the stage for autophagosome formation. Biochem Soc Trans 42: 1327-1334.

Wittke, S., Lewke, N., Muller, S., and Johnsson, N. (1999) Probing the molecular environment of membrane proteins in vivo. Mol Biol Cell 10: 2519-2530.

Wu, F., Watanabe, Y., Guo, X.-Y., Qi, X., Wang, P., Zhao, H.-Y., et al. (2015) Structural Basis of the Differential Function of the Two C. elegans Atg8 Homologs, LGG-1 and LGG-2, in Autophagy. Mol Cell 60: 914-929.

Xie, Z., Nair, U., and Klionsky, D.J. (2008) Atg8 controls phagophore expansion during autophagosome formation. Mol Biol Cell 19: 3290-3298.

$\mathrm{Xu}, \mathrm{C}$. , and Min, J. (2011) Structure and function of WD40 domain proteins. Protein Cell 2: 202-214.

Yamaguchi, M., Noda, N.N., Nakatogawa, H., Kumeta, H., Ohsumi, Y., and Inagaki, F. (2010) Autophagy-related Protein 8 (Atg8) Family Interacting Motif in Atg3 Mediates the Atg3-Atg8 Interaction and Is Crucial for the Cytoplasm-to-Vacuole Targeting Pathway. Journal of Biological Chemistry 285: 29599-29607.

Yamaguchi, M., Noda, N.N., Yamamoto, H., Shima, T., Kumeta, H., Kobashigawa, Y., et al. (2012) Structural insights into Atg10-mediated formation of the autophagyessential Atg12-Atg5 conjugate. Structure 20: 1244-1254.

Yamamoto, H., Fujioka, Y., Suzuki, S.W., Noshiro, D., Suzuki, H., Kondo-Kakuta, C., et al. (2016) The Intrinsically Disordered Protein Atg13 Mediates Supramolecular Assembly of Autophagy Initiation Complexes. Developmental Cell 38: 86-99.

Yamamoto, H., Kakuta, S., Watanabe, T.M., Kitamura, A., Sekito, T., Kondo-Kakuta, C., et al. (2012) Atg9 vesicles are an important membrane source during early steps of autophagosome formation. J Cell Biol 198: 219-233.

Yao, Z., Delorme-Axford, E., Backues, S.K., and Klionsky, D.J. (2015) Atg41/Icy2 regulates autophagosome formation. Autophagy 11: 2288-2299.

Yen, W.-L., and Klionsky, D.J. (2008) How to live long and prosper: autophagy, mitochondria, and aging. Physiology (Bethesda) 23: 248-262.

Yen, W.-L., Legakis, J.E., Nair, U., and Klionsky, D.J. (2007) Atg27 is required for autophagy-dependent cycling of Atg9. Mol Biol Cell 18: 581-593.

Yuga, M., Gomi, K., Klionsky, D.J., and Shintani, T. (2011) Aspartyl aminopeptidase is imported from the cytoplasm to the vacuole by selective autophagy in Saccharomyces cerevisiae. Journal of Biological Chemistry 286: 13704-13713. 
Zheng, J.-X., Li, Y., Ding, Y.-H., Liu, J.-J., Zhang, M.-J., Dong, M.-Q., et al. (2017) Architecture of the ATG2B-WDR45 complex and an aromatic Y/HF motif crucial for complex formation. Autophagy 13: 1870-1883. 


\section{Supplementary Figures}
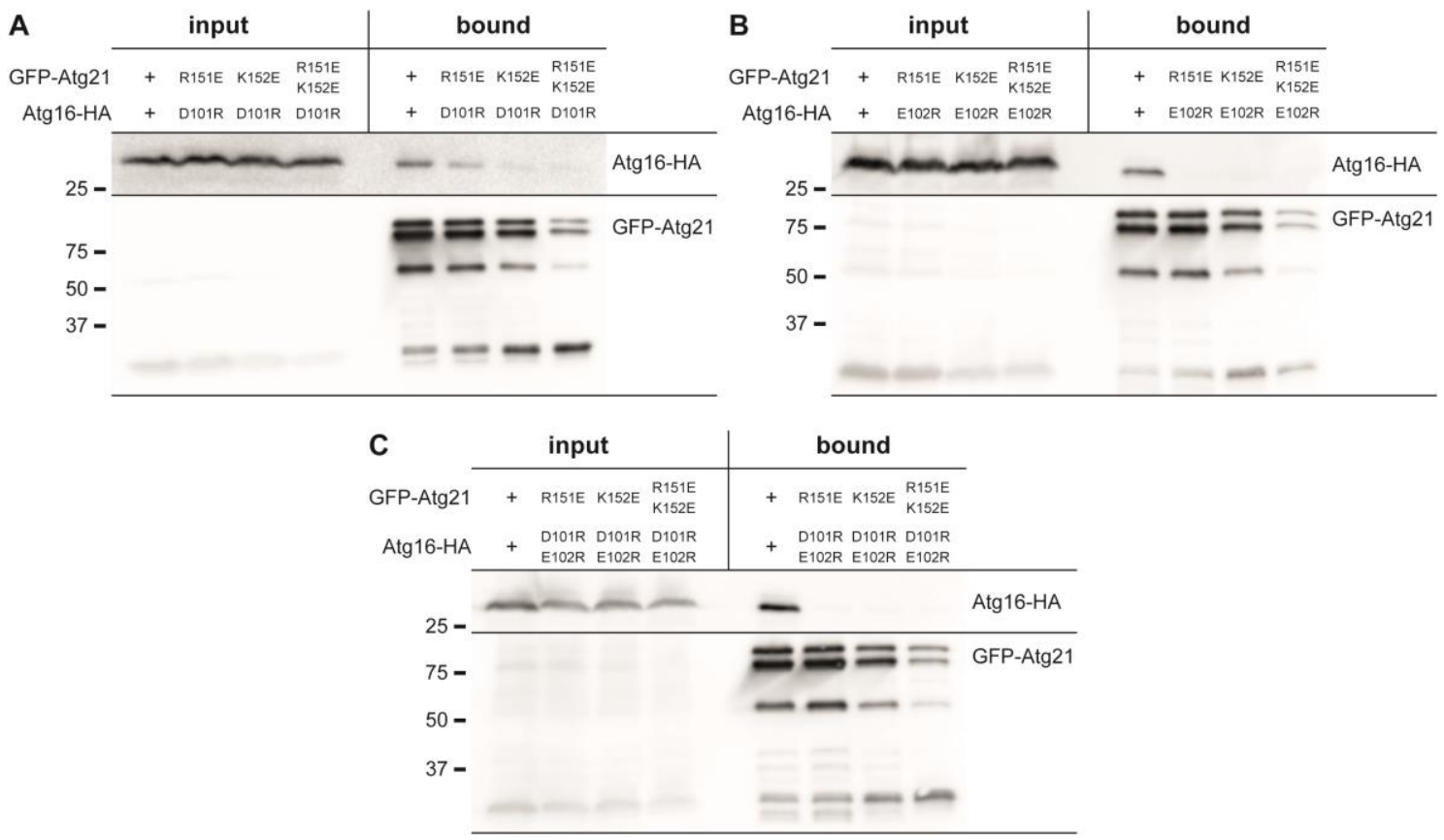

Figure 7.1: Only the simultaneous charge change of Atg21 R151 (R151E) and Atg16 D101 (D101R) partially restores the interaction of both proteins

Analysis of the effect of the Atg21 R151E and K152E single and double mutant on the interaction with Atg16 D101R and E102R single and double mutants using the GFP-TRAP approach. In an atg21S atg16 $\Delta$ strain the plasmid-encoded bait (GFP, GFP-ATG21 or its mutants) and prey (ATG16-HA or its mutants) were overexpressed using the MET25 and CUP1 promoter respectively. Cells were grown in selection medium without L-methionine to early stationary phase ( $\left(\mathrm{OD}_{600} 2\right)$, osmotically lysed and incubated with GFP-TRAP beads. Input and bound fractions were analyzed by Western-Blot. The molecular weight marker is depicted in $\mathrm{kDa}$. 
A

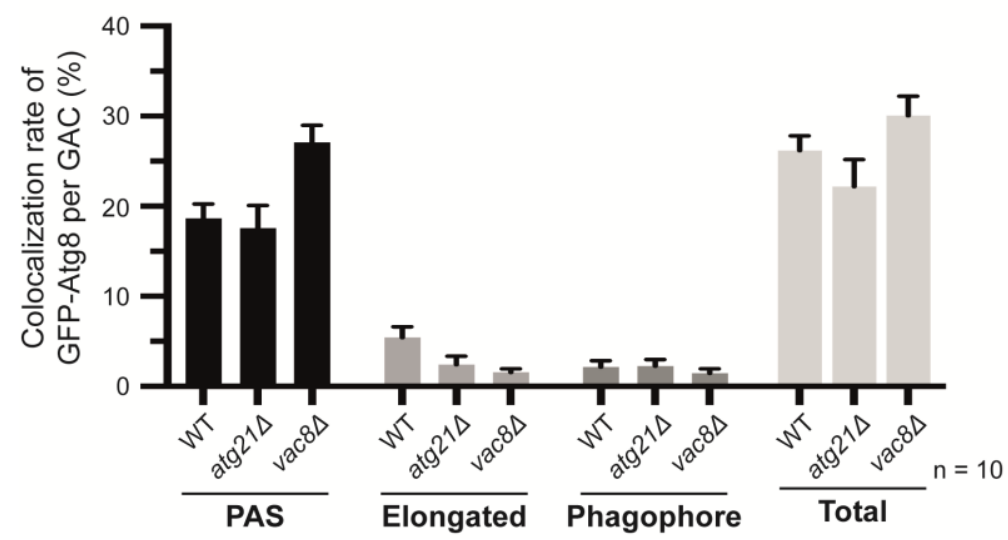

B

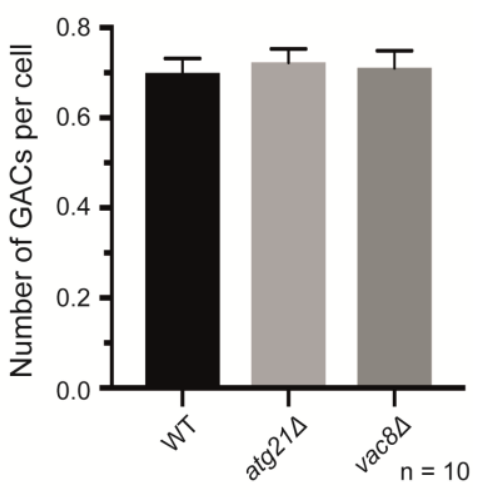

Figure 7.2: Colocalization of GFP-Atg8 with GACs in the 3xtagBFP-PHO8 expressing WT, atg21 4 and vav8 $\Delta$ strains

Determination of the colocalization of GFP-Atg8 and the Ape1-RFP positive GACs in the 3xtagBFP-

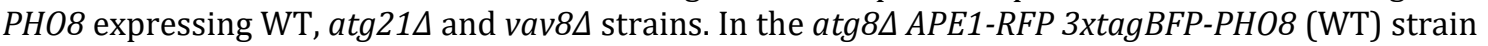
either ATG21 (atg21 ) or VAC8 (vac8D) was deleted. In these strains, plasmid-encoded GFP-ATG8 and APE1 were expressed using the ATG8 and CUP1 promoter respectively. The cells were grown over night in selection medium with $100 \mu \mathrm{M} \mathrm{CuSO}_{4}$. Stationary cells $\left(\mathrm{OD}_{600} 4-5\right)$ were starved for $1 \mathrm{~h}$ in SD-N medium and analyzed by fluorescence microscopy using the DeltaVision microscope equipped with GFP and mCherry filter sets. In total, 10 images (n) with at least 540 cells per strain were analyzed. The number of GACs per cell (B) and their colocalization with GFP-Atg8 (A) was determined per image. The shape of the colocalizing GFP-Atg8 signal was grouped into dot-like (PAS), slightly elongated and cup-like (Phagophore) structures. The colocalization rate of each group and of the sum of all groups (Total) was calculated. 

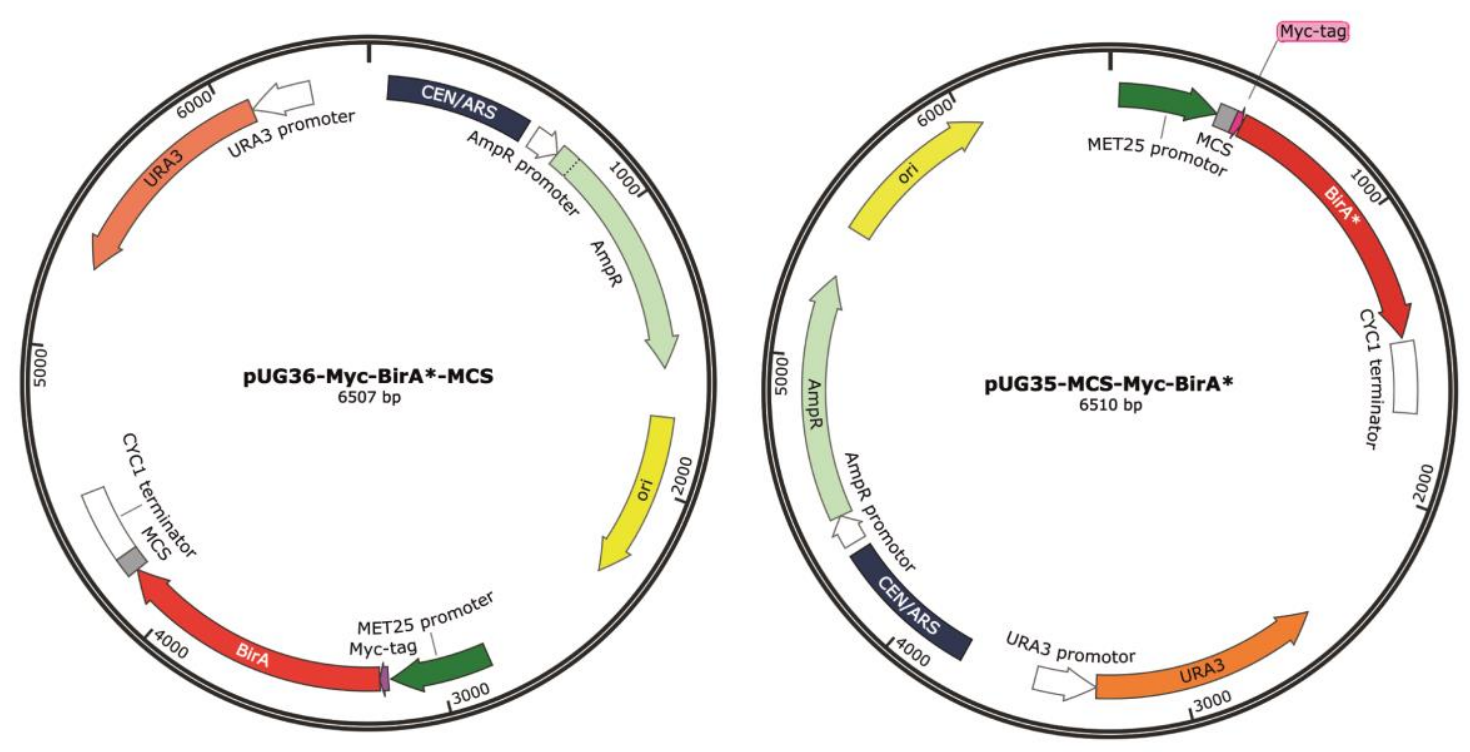

Figure 7.3: Plasmid maps of the BirA* fusion vectors pUG36-Myc-BirA*-MCS and pUG35-MCSMyc-BirA*

The plasmids comprise the Myc (pink) tagged BirA* gene (red) five prime (pUG36-Myc-BirA*-MCS) or three prime (pUG35-MCS-Myc-BirA*) to a multiple cloning site (MCS; grey). The gene is under the control of the MET25 promoter (green) and the CYC1 terminator (white). The AmpR cassette (light green) and the URA3 gene (orange) serve as selection markers. For replication the plasmids comprise the $S$. cerevisiae CEN6 centromere fused to an autonomously replicating sequence (CEN/ARS; dark blue) and an origin of replication (ori; yellow). 


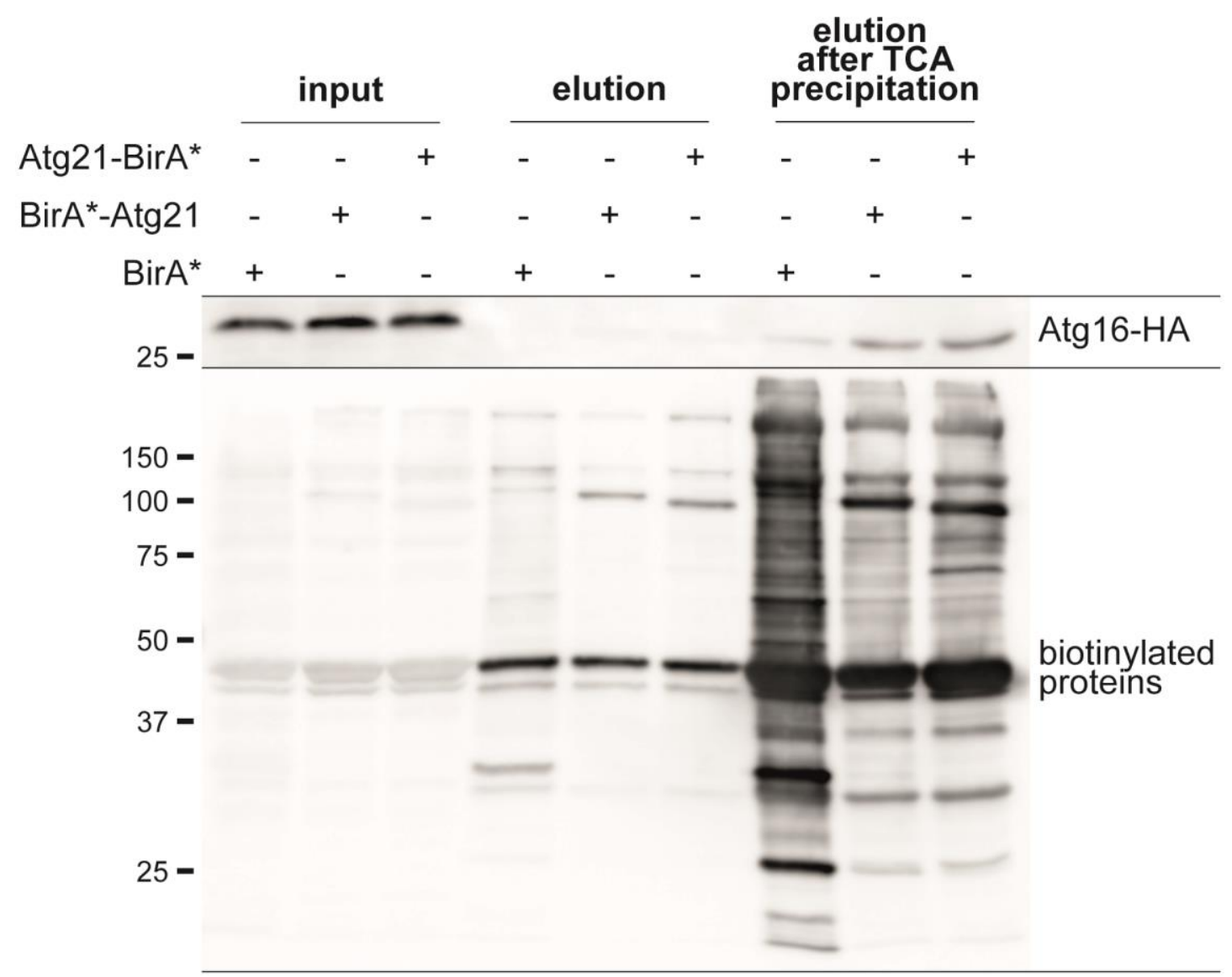

Figure 7.4: Pattern of the isolated biotinylated proteins from cells carrying BirA*-Atg21, Atg21-BirA* or BirA* alone

In an atg21 $\operatorname{atg} 16 \Delta$ strain, plasmid-encoded $A T G 16-H A$ was expressed in combination with BirA*ATG21, ATG21-BirA* or free BirA*. The expression was controlled by the CUP1 and MET25 promoter respectively. Cells were grown over night in selection medium without L-methionine supplemented with $10 \mu \mathrm{M}$ biotin. Early stationary cells $\left(\mathrm{OD}_{600} 2\right)$ were processed according to the BioID workflow. Biotinylated proteins were isolated using $0.2 \mathrm{ml}$ gravity flow Strep-Tactin ${ }^{\circledR}$ Sepharose ${ }^{\circledR}$ columns (iba) and precipitated in presence of $10 \%(\mathrm{w} / \mathrm{v})$ TCA. Input, elution and precipitated elution fractions were analyzed by Western-Blot. For the detection of biotinylated proteins, the PVDF membrane was blocked in 3\% (w/v) BSA in TBST and decorated with a Strep-Tactin-HRP conjugate. The molecular weight marker is depicted in $\mathrm{kDa}$. 


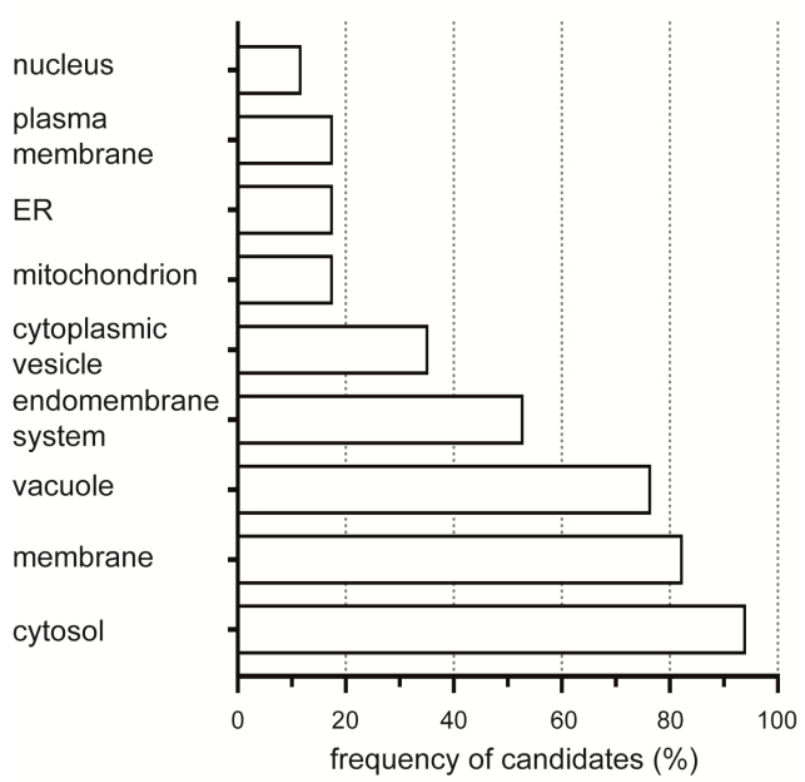

Figure 7.5: Spatial grouping of the potential interaction partners of Atg21 according to their reported association with distinct compartments in the cell

The 17 proteins identified as proximal/interacting proteins of BirA*-Atg21 using the SILAC-based BioID approach were grouped according to their reported association with distinct compartments in the cell with the help of the Gene Ontology Slim Mapper of the Saccharomyces Genome Database (https://www.yeastgenome.org). The number of candidates per group is reflected by the frequency of candidates in one group in comparison to the total number of candidates in \%. Corresponding candidates are listed. 


\section{Acknowledgement}

Zuallererst und ganz besonders möchte ich mich bei Prof. Dr. Michael Thumm für die Möglichkeit zur Promotion und der Anfertigung dieser Arbeit bedanken. Weiterhin bedanke ich mich für die viele Unterstützung in sämtlichen Bereichen und die Erstellung des Erstgutachtens.

Den weiteren Mitgliedern meines Thesis Committees, Prof. Dr. Gerhard Braus und Prof. Dr. Ralf Ficner, möchte ich ebenfalls danken für die Unterstützung des Fortschritts dieser Arbeit durch Diskussionen und Anregungen. Darüber hinaus möchte ich mich bei Prof. Dr. Gerhard Braus für die Übernahme des Zweitgutachtens bedanken.

Unseren Kooperationspartnern Dr. Karin Kühnel und Dr. Janina Metje danke ich für die Bereitstellung der Kristallstruktur von Atg21 und Atg16 sowie für den wissenschaftlichen Austausch zu dem gemeinsamen Forschungsprojekt.

Ein besonderer Dank gilt auch Dr. Oliver Valerius und Dr. Kerstin Schmitt (Institut für Mikrobiologie und Genetik, Georg-August-universität Göttingen) für die Durchführung der LC-MS Analysen und Auswertung der Daten. Darüber hinaus möchte ich ihnen für die Bereitstellung des Protokolls zum BioID Assay und die vielen hilfreichen Ratschläge zur Durchführung bedanken. In diesem Zusammenhang möchte ich mich auch bei Olaf Bernhard (Institut für Zellbiochemie, Universitätsmedizin Göttingen) für die Aufarbeitung der Proben für die LC-MS Analysen bedanken.

Dr. Narain Karedla (Abteilung Biophysik/ Komplexe Systeme, III. Physikalisches Institut, Georg-August-Universität Göttingen) danke ich für die Durchführung der FCCS Messungen und der sich anschließenden Berechnungen.

Ganz besonders möchte ich mich auch bei Rosi bedanken für die enorme Unterstützung, besonders während des Zusammenschreibens der Arbeit.

Der gesamten AG Thumm: Petra, Rosi, Flo, Lisa Ma., Steffi, Hussein und Matt aber auch den ehemaligen Kollegen Lisa Mi., Marco und Peter möchte ich von Herzen danken für das tolle humorvolle Laborklima und die vielen gemeinsamen 
Aktivitäten im und außerhalb des Labors. Insbesondere danke ich Petra für die angenehme Zusammenarbeit und die vielen aufmunternden Gespräche!

Mein herzlichster Dank geht an meinen Freund Matthias Röhr, an meine Familie, insbesondere meine Eltern, und an alle weiteren Freunde für die Motivation, den Rückhalt in allen Lebenslagen, die unglaubliche Unterstützung und den festen Glauben an mich! 